Éscuela Técnica Superior de Ingenieros Industriales

\title{
ESTUDIO DE LA VIABILIDAD DEL RECICLADO MECÁNICO DEL POLI(ÁCIDO LÁCTICO) Y SUS NANOCOMPOSITES
}

\author{
TESIS DOCTORAL
}

Freddys Beltrán González

Máster en Ingeniería Química

Madrid 2018 

Departamento de Ingeniería Química Industrial y Medio Ambiente Escuela Técnica Superior de Ingenieros Industriales

\title{
ESTUDIO DE LA VIABILIDAD DEL RECICLADO MECÁNICO DEL POLI(ÁCIDO LÁCTICO) Y SUS NANOCOMPOSITES
}

\author{
Freddys Beltrán González \\ Máster en Ingeniería Química
}

\begin{abstract}
Directores
Joaquín Martínez Urreaga

María Ulagares de la Orden

Doctor en Ciencias Químicas

Doctora en Ciencias Químicas
\end{abstract}

Madrid, 2018 


\section{ÍNDICE}

Índice $\quad$ iv

Lista de Figuras vii

Lista de Tablas $\quad$ xi

Agradecimientos xiii

Resumen xiv

$\begin{array}{lll}\text { Abstract } & \text { xvi }\end{array}$

Publicaciones Derivadas $\quad$ xviii

1 Introducción 1

1.1 Estructura de la tesis 1

1.2 Planteamiento del problema y antecedentes 1

1.3 Enfoque de la tesis y objetivos 9

2 Marco teórico 11

2.1 Poli(ácido láctico) 11

2.1.1 Producción industrial del PLA 11

2.1.2 Estructura del PLA 12

2.1.3 Aplicaciones del PLA 13

2.2 Nanocomposites basados en PLA 15

2.2.1 Arcillas: montmorillonita y haloisita 15

2.2.2 Producción de los nanocomposites 16

2.2.3 Aplicaciones de los nanocomposites 17

2.3 Reciclado mecánico 17

$\begin{array}{ll}2.3 .1 \text { Etapas del reciclado mecánico } & 18\end{array}$

3 Metodología 20

$\begin{array}{ll}3.1 \text { Materiales } & 20\end{array}$

3.2 Obtención de materiales vírgenes y reciclados 21 
3.3 Mejora de las propiedades del PLA reciclado

3.4 Modificación de la haloisita con 3-aminopropiltrietoxisilano 24

3.5 Ensayos de absorción de agua y degradación hidrolítica 24

3.6 Técnicas de caracterización 24

4 Efecto del reciclado mecánico en la estructura y propiedades del PLA y sus nanocomposites 28

4.1 Viscosidad intrínseca de los materiales vírgenes y reciclados 28

4.2 Morfología de los nanocomposites vírgenes y reciclados 32

4.3 Estructura de los materiales vírgenes y reciclados 35

4.4 Estabilidad térmica de los materiales vírgenes y reciclados 43

4.5 Propiedades ópticas de los materiales reciclados 46

4.6 Microdureza de los materiales reciclados 49

4.7 Propiedades de transporte de los materiales reciclados 51

5 Efecto del reciclado en la degradación hidrolítica del PLA y sus nanocomposites 54

5.1 Cinética de la absorción de agua de los materiales reciclados 54

5.2 Estudio de los productos de degradación mediante espectroscopía UV-Vis $\quad 61$

5.3 Evolución de la viscosidad intrínseca durante la inmersión de los materiales $\begin{array}{ll}\text { reciclados } & 64\end{array}$

5.4 Cambios estructurales durante la degradación hidrolítica 68

5.5 Evolución de la estabilidad térmica durante la inmersión de los materiales reciclados 91

5.6 Evolución de la microdureza durante la inmersión de los materiales reciclados 93

6 Mejora de propiedades del PLA reciclado 97

6.1 Mejora de prestaciones de PLA reciclado mediante extrusión reactiva 97

6.1.1 Viscosidad intrínseca del PLA reciclado mejorado mediante extrusión reactiva 97

6.1.2 Efecto de la extrusión reactiva en la estructura del PLA reciclado 100

6.1.3 Efecto de la extrusión reactiva en la estabilidad térmica del PLA reciclado 103

6.1.4 Efecto de la extrusión reactiva en las propiedades ópticas del PLA reciclado 104

6.1.5 Efecto de la extrusión reactiva en la microdureza del PLA reciclado 106

6.2 Mejora de prestaciones de PLA reciclado mediante adición de arcillas $\quad 106$

6.2.1 Viscosidad intrínseca del PLA mejorado mediante adición de arcillas 107

6.2.2 Efecto de la adición de arcillas en la estructura del PLA reciclado 107

6.2.3 Efecto de la adición de arcillas en la estabilidad térmica del PLA reciclado $\quad 110$

6.2.4 Efecto de la adición de arcillas en las propiedades ópticas del PLA reciclado 111 
6.2.5 Efecto de la adición de arcillas en la microdureza del PLA reciclado

7 Conclusiones y trabajos futuros 114

$\begin{array}{ll}7.1 \text { Conclusiones } & 114\end{array}$

$\begin{array}{ll}\text { 7.2 Trabajos futuros y recomendaciones } & 118\end{array}$

8 Referencias 119

$\begin{array}{ll}\text { Anexo A: Publicaciones } & 135\end{array}$ 


\section{LISTA DE FIGURAS}

Fig. 1. Estructura química del PLA [10].

Fig. 2. Métodos para la obtención de PLA de alto peso molecular. Adaptado de [10] 12

Fig. 3. Modificación orgánica de la arcilla Cloisite C30B. 20

Fig. 4. Esquema de la simulación de los diferentes procesos de reciclado. 21

Fig. 5. Esquema del proceso de mejora de propiedades del PLA reciclado. 23

Fig. 6. Valores de viscosidad intrínseca del PLA sometido a diferentes procesos de reciclado mecánico. $\quad 29$

Fig. 7. Viscosidad intrínseca de los diferentes nanocomposites basados en PLA. $\quad 31$

Fig. 8. Fotografías TEM del PLAV-C30 (a) y PLAR-C30 (b). 33

Fig. 9. Difractogramas de la arcilla C30, y de los nanocomposites PLA-C30 virgen y reciclados $\left(2 \theta<8^{\circ}\right)$. 34

Fig. 10. Fotografías TEM del PLAV-Ha (a) y PLAR-Ha (b). 35

Fig. 11. Espectros FTIR del PLA virgen y sometido a diferentes procesos de reciclado.

Fig. 12. Ampliación de la banda de absorción centrada a $1755 \mathrm{~cm}^{-1}$ del PLAV y PLARLH.

Fig. 13. Espectro FTIR de los nanocomposites de PLA sometidos a diferentes procesos $\begin{array}{ll}\text { de reciclado. } & 38\end{array}$

Fig. 14. Difractogramas del PLAV, PLAR y PLARL $\left(6^{\circ}<2 \theta<40^{\circ}\right)$. 39

Fig. 15. Difractogramas del nanocomposite PLA-C30 sometido a diferentes procesos de reciclado mecánico $\left(6^{\circ}<2 \theta<40^{\circ}\right)$.

Fig. 16. Barridos del segundo calentamiento del PLA sometido a diferentes procesos de reciclado.

Fig. 17. Barridos del segundo calentamiento del nanocomposite PLA-C30 sometido a diferentes procesos de reciclado.

Fig. 18. Barridos del segundo calentamiento del nanocomposite PLA-Ha sometido a diferentes procesos de reciclado. 
Fig. 19. Termogramas del PLA sometido a diferentes procesos de reciclado mecánico.

Fig. 20. Termogramas de los nanocomposites PLA-C30 sometido a diferentes procesos de reciclado mecánico.

Fig. 21. Espectros UV-Vis del PLA sometido a diferentes procesos de reciclado mecánico.

Fig. 22. Espectros UV-Vis del nanocomposite PLA-C30 sometido a diferentes procesos de reciclado mecánico.

Fig. 23. Espectros UV-Vis del nanocomposite PLA-Ha sometido a diferentes procesos de reciclado mecánico.

Fig. 24. Valores de microdureza de los diferentes materiales.

Fig. 25. Coeficiente de permeabilidad de los diferentes materiales frente al $\mathrm{O}_{2}, \mathrm{~N}_{2}$ y $\mathrm{CO}_{2}$.

Fig. 26. Curvas de absorción de agua, a $37^{\circ} \mathrm{C}$, del PLA sometido a diferentes procesos de reciclado mecánico.

Fig. 27. Ajuste de los datos de absorción de agua, a $37^{\circ} \mathrm{C}$, del PLA sometido a diferentes procesos de reciclado mecánico, utilizando la ecuación 7 .

Fig. 28. Curvas de absorción de agua, a $37^{\circ} \mathrm{C}$, de los nanocomposites PLA-C30 sometidos a diferentes procesos de reciclado.

Fig. 29. Ajuste de los datos de absorción de agua, a $58^{\circ} \mathrm{C}$, del PLAV y PLAR 60

Fig. 30. Mecanismo de degradación hidrolítica del PLLA en medio básico o neutro. 62

Fig. 31. Espectros UV-Vis del líquido de inmersión, a diferentes temperaturas, del PLA

Fig. 32. Espectros UV-Vis del líquido de inmersión, después de 6 días de inmersión a 58 ${ }^{\circ} \mathrm{C}$ del nanocomposite PLA-C30.

Fig. 33. Evolución de la viscosidad intrínseca, a $37{ }^{\circ} \mathrm{C}$, del PLA virgen y sometido a diferentes procesos de reciclado.

Fig. 34. Evolución de la viscosidad intrínseca, a $37^{\circ} \mathrm{C}$, del nanocomposite PLA-C30 sometido a diferentes procesos de reciclado.

Fig. 35. Evolución de la viscosidad intrínseca, a $37{ }^{\circ} \mathrm{C}$, del nanocomposite PLA-Ha sometido a diferentes procesos de reciclado.

Fig. 36. Espectros FTIR del PLAV sin sumergir, y del PLAV, PLAR y PLARL después de 84 días de inmersión a $37{ }^{\circ} \mathrm{C}$.

Fig. 37. Espectros del PLAV-C30 y el PLAR-C30 antes y después de 84 días de inmersión a $37^{\circ} \mathrm{C}$.

Fig. 38. Difractogramas del PLA, sometido a diferentes procesos de reciclado, sumergido a $37^{\circ} \mathrm{C}$. 
Fig. 39. Difractogramas del nanocomposite PLA-C30, sometido a diferentes procesos de reciclado, sumergido a $37^{\circ} \mathrm{C}$.

Fig. 40. Primer barrido de calentamiento del PLAV, PLAR y PLARL sumergido a $37^{\circ} \mathrm{C}$.

Fig. 41. Primer barrido de calentamiento del nanocomposite PLA-C30, sometido a diferentes procesos de reciclado, sumergido a $37^{\circ} \mathrm{C}$.

Fig. 42. Primer barrido de calentamiento del nanocomposite PLA-Ha, sometido a diferentes procesos de reciclado, sumergido a $37^{\circ} \mathrm{C}$.

Fig. 43. Espectros FTIR de las diferentes muestras antes y después de 13 días de inmersión a $58^{\circ} \mathrm{C}$.

Fig. 44. Difractogramas del PLAV. PLAV-C30 y PLAV-Ha antes y después de 13 días de inmersión a $58^{\circ} \mathrm{C}$.

Fig. 45. Banda de absorción de $920 \mathrm{~cm}^{-1}$ del PLA con diferentes formas cristalinas. 83

Fig. 46. Picos de reflexión (110)/(200) de los diferentes materiales después de 2 y 13 días de inmersión a $58^{\circ} \mathrm{C}$.

Fig. 47. Primer barrido de calentamiento del PLA, virgen y reciclado, sumergido a $58^{\circ} \mathrm{C}$.

Fig. 48. Primer barrido de calentamiento del nanocomposite PLA-C30, virgen y reciclado, sumergido a $58^{\circ} \mathrm{C}$.

Fig. 49. Primer barrido de calentamiento del nanocomposite PLA-Ha, virgen y reciclado, sumergido a $58^{\circ} \mathrm{C}$.

Fig. 50. Evolución de la $\mathrm{T}_{10}$ de los materiales sumergidos a $37^{\circ} \mathrm{C}$

Fig. 51. Evolución de la microdureza de los materiales sumergidos a $37^{\circ} \mathrm{C}$.

Fig. 52. Viscosidad intrínseca del PLA reciclado extruido mejorado mediante extrusión reactiva.

Fig. 53. Mecanismo de extensión de cadena del PLA con compuestos epoxi. Adaptado de [111].

Fig. 54. Esquema de la reacción del PLA con peróxidos. Adaptado de [110].

Fig. 55. Espectros FTIR del PLA reciclado mejorado mediante extrusión reactiva. 101

Fig. 56. Ampliación de la banda de $1750 \mathrm{~cm}^{-1}$ del PLA reciclado mejorado mediante extrusión reactiva.

Fig. 57. Barridos de segundo calentamiento y de enfriamiento del PLA reciclado mejorado mediante extrusión reactiva.

Fig. 58. Termogramas del PLA mejorado mediante extrusión reactiva.

Fig. 59. Espectros UV-Vis del PLA mejorado mediante extrusión reactiva. 
Fig. 61. Viscosidad intrínseca del PLA reciclado mejorado mediante adición de arcillas.

Fig. 62. Espectros FTIR del PLA reciclado mejorado mediante la adición de arcillas.108 Fig. 63. Barridos de segundo calentamiento del PLA reciclado mejorado mediante adición de arcillas.

Fig. 64. Termogramas del PLA mejorado mediante adición de arcillas

Fig. 65. Espectros UV-Vis del PLA reciclado mejorado mediante adición de arcillas.112

Fig. 66. Microdureza del PLA reciclado mejorado mediante adición de arcillas. 


\section{LISTA DE TABLAS}

Tabla 1. Materiales obtenidos en los diferentes procesos de reciclado simulado

Tabla 2. Materiales obtenidos según los diferentes métodos para la recuperación de propiedades del PLA reciclado

Tabla 3. Bandas de absorción características del PLA.

Tabla 4. Resultados de DSC (segundo barrido de calentamiento) de los diferentes materiales.

Tabla 5. Temperaturas características de TGA de los diferentes materiales.

Tabla 6. Transmisión de luz de los materiales vírgenes y reciclados.

Tabla 7. Parámetros de absorción de agua, a $37^{\circ} \mathrm{C}$, de los diferentes materiales vírgenes y reciclados.

Tabla 8. Parámetros de absorción de agua, a $58^{\circ} \mathrm{C}$, de los diferentes materiales vírgenes y reciclados.

Tabla 9. Evolución de la viscosidad intrínseca de los diferentes materiales sumergidos a $58^{\circ} \mathrm{C}$.

Tabla 10. Evolución de las transiciones térmicas durante la inmersión a $37{ }^{\circ} \mathrm{C}$ del PLA sometido a diferentes procesos de reciclado.

Tabla 11. Evolución de las transiciones térmicas durante la inmersión a $37{ }^{\circ} \mathrm{C}$ del nanocomposite PLA-C30 sometido a diferentes procesos de reciclado.

Tabla 12. Evolución de las transiciones térmicas durante la inmersión a $37{ }^{\circ} \mathrm{C}$ del nanocomposite PLA-Ha sometido a diferentes procesos de reciclado.

Tabla 13. Valores de cristalinidad estimados mediante FTIR y XRD de las diferentes muestras sumergidas a $58^{\circ} \mathrm{C}$.

Tabla 14. Espacio reticular de la reflexión $(110) /(200)$ y proporción de la forma $\alpha$ de las muestras después de 2 y 13 días de inmersión a $58^{\circ} \mathrm{C}$.

Tabla 15. Evolución de las entalpías y de la cristalinidad del PLA, virgen y reciclado, sumergido a $58^{\circ} \mathrm{C}$.

Tabla 16. Evolución de las entalpías y de la cristalinidad del nanocomposite PLA-C30, virgen y reciclado, sumergido a $58^{\circ} \mathrm{C}$. 
Tabla 17. Evolución de las entalpías y de la cristalinidad del nanocomposite PLA-Ha, virgen y reciclado, sumergido a $58^{\circ} \mathrm{C}$.

Tabla 18. Evolución de $\mathrm{T}_{10}$ de los materiales sumergidos a $58^{\circ} \mathrm{C}$.

Tabla 19. Evolución de la microdureza de los diferentes materiales sumergidos a $58{ }^{\circ} \mathrm{C}$.

Tabla 20. Resultados de DSC (segundo barrido de calentamiento) del PLA reciclado mejorado mediante extrusión reactiva.

Tabla 21. Temperaturas características de TGA del PLA mejorado mediante extrusión reactiva.

Tabla 22. Transmisión de luz del PLA mejorado mediante extrusión reactiva.

105

Tabla 23. Resultados de DSC (segundo barrido de calentamiento) del PLA reciclado mejorado mediante la adición de arcillas.

Tabla 24. Temperaturas características de TGA del PLA mejorado mediante adición de arcillas.

Tabla 25. Transmisión de luz del PLA reciclado mejorado mediante adición de arcillas. 


\section{AGRADECIMIENTOS}

En primer lugar, me gustaría agradecer a mis padres Fredys y Aura, sin cuyo esfuerzo y sacrificio nada de lo que he logrado hasta hoy hubiese sido posible. A mi pareja, Yarima, por acompañarme y apoyarme siempre, incluso en los momentos más difíciles.

A mis directores, Mariula y Joaquín, por la oportunidad que me han dado para trabajar con ellos, por sus consejos y por su buena labor a la hora de dirigir esta tesis.

Me gustaría agradecer también a los profesores y PAS del Laboratorio de Química I de la E.T.S.I. Industriales de la Universidad Politécnica de Madrid por su colaboración, su buena disposición y su compañía durante la realización de este trabajo. Al profesor Vicente Lorenzo por su ayuda y sus consejos, así como al personal del Instituto de Ciencia y Tecnología de Polímeros del CSIC, por su colaboración. A los estudiantes que han pasado por el laboratorio para realizar sus TFG y TFM, que con su compañía hicieron más amenos estos casi 4 años.

Por último, pero no por eso menos importantes, a mis amigos Claudio, Alejandro, Héctor y Jürgen. Porque a pesar de la distancia siempre han estado allí para aconsejarme y ayudarme. Parece mentira que haya pasado ya más de una década desde que estábamos empezando nuestro camino en la Universidad Simón Bolívar, cuando obtener siquiera el título de Ingeniero parecía algo muy lejano.

Como diría Cerati: "¡Gracias totales!” 


\section{RESUMEN}

El poli(ácido láctico) (PLA) es un bioplástico producido a partir de fuentes renovables que, debido a sus buenas prestaciones, ha ganado interés como candidato para reemplazar a los plásticos producidos a partir de combustibles fósiles en aplicaciones como el envasado de alimentos. Sin embargo, el uso creciente de este bioplástico podría traer problemas ambientales y sociales, relacionados con la gestión adecuada de la gran cantidad de residuos que podrían generarse y con la superficie necesaria para cultivar la materia prima empleada en la producción del PLA. Por este motivo, es interesante evaluar alternativas que permitan la valorización adecuada de los residuos de PLA y la reducción del consumo de materias primas.

Por ello, uno de los objetivos principales de este trabajo es el estudio de la viabilidad del reciclado mecánico del PLA y sus nanocomposites con arcillas, analizando el efecto de este proceso en las propiedades de los plásticos reciclados. Para cumplir este

objetivo se ha sometido al PLA, y sus nanocomposites con montmorillonita y haloisita, a diferentes procesos de reciclado, que pueden incluir: una etapa de extrusión y moldeo por compresión; un envejecimiento acelerado (utilizado para simular la degradación durante la vida útil) que consta de etapas de degradación fotoquímica, térmica e hidrolítica; un proceso de lavado intenso y, finalmente, una etapa de reprocesado por extrusión y moldeo por compresión. Los materiales resultantes han sido caracterizados mediante diferentes técnicas experimentales, incluyendo viscosimetría, espectroscopía infrarroja y UVvisible, calorimetría diferencial de barrido, termogravimetría, microscopía electrónica (barrido y trasnmisión) y difracción de rayos X.

El reciclado mecánico ocasiona la degradación del PLA. Los resultados obtenidos indican que la magnitud de esta degradación depende de las condiciones del proceso, ya que, por ejemplo, la inclusión de una etapa de lavado enérgico produce descensos del 20 $\%$ en la viscosidad intrínseca, lo que complica la procesabilidad del plástico reciclado. Esta reducción de la viscosidad del PLA se traduce además en una mayor habilidad para cristalizar, una menor estabilidad térmica y un ligero descenso en las propiedades de barrera frente a gases del plástico reciclado. No obstante, el descenso de las propiedades debido al reciclado mecánico es en general pequeño, lo que sugiere que el plástico reciclado podría tener una segunda vida.

En los nanocomposites con las diferentes arcillas, se observa también la degradación del PLA después de los diferentes procesos de reciclado. Sin embargo, el 
reprocesado ocasiona una mejor dispersión de las arcillas en la matriz polimérica, lo que conlleva en este caso incluso mejores propiedades mecánicas, térmicas y de barrera en los nanocomposites reciclados.

El descenso de la viscosidad intrínseca, y de algunas propiedades, del PLA podría afectar negativamente al interés comercial por el plástico reciclado $\mathrm{y}$, por tanto, a la reciclabilidad del material. Por ello, el otro objetivo de esta tesis es el estudio de alternativas para mejorar las prestaciones del PLA reciclado, sin encarecer excesivamente el coste del proceso. Para ello, se ha sometido un grado comercial de PLA a un proceso de reciclado que incluye una etapa de procesado en fundido, un envejecimiento acelerado, una etapa de lavado y un segundo procesado en fundido. Es en esta segunda etapa de procesado cuando se añaden diferentes aditivos (como peróxido de dicumilo y un extensor de cadena), o diferentes arcillas (montmorillonita y haloisita) para mejorar las propiedades del material reciclado.

Los resultados indican que tanto adición del peróxido y del extensor de cadena, como la adición de las diferentes arcillas, ocasionan un incremento de la viscosidad intrínseca del material, así como una mejora de las propiedades mecánicas y térmicas del PLA reciclado.

En resumen, se puede decir que el reciclado mecánico ocasiona la degradación del PLA, y un pequeño descenso en sus propiedades. Este descenso es pequeño en general, y el material reciclado podría ser utilizado nuevamente, incluso en aplicaciones de envasado. Sin embargo, es posible obtener PLA reciclado con mejores prestaciones sin aumentar de forma importante el coste del proceso, lo que debería mejorar la reciclabilidad de este bioplástico. 


\section{ABSTRACT}

Poly(lactic acid) (PLA) is a bioplastic produced from renewable resources. Due to its good properties, PLA has attracted interest as an alternative to fossil-fuel based polymers in several applications, such as food packaging. However, the increasing use of this bioplastic might generate some environmental and social problems, related to the large amount of wastes and to the cropland needed to produce the raw materials used in PLA production. Therefore, is interesting to evaluate alternatives that allow the valorization of PLA wastes and the reduction of the consumption of raw materials.

Consequently, one of the main aims of this work is to study the feasibility of mechanical recycling of PLA and its nanocomposites with clays, analyzing the effect of this process on the properties of the recycled plastics. To achieve this objective, PLA and its nanocomposites were subjected to different recycling processes, which might include: an extrusion and compression molding stage; an accelerated aging step (to simulate the degradation during service life), which consisted of photochemical, thermal and hygrothermal aging processes; a demanding washing step and, finally, a second reprocessing by extrusion and compression molding. The resulting materials were then characterized by viscosimetry, UV-Vis and infrared spectroscopy, differential scanning calorimetry, thermogravimetric analysis, transmission and scanning electron microscopies and $\mathrm{X}$-ray diffraction.

Mechanical recycling causes the degradation of PLA. The results point out that the intensity of this degradation depends on the process conditions, since the inclusion of a washing step led to a $20 \%$ decrease of the intrinsic viscosity. This degradation results in an increased crystallization ability and reduced thermal stability and gas barrier properties. However, the decrease of the properties due to the mechanical recycling processes is small, which suggests that PLA can be subjected to mechanical recycling. Regarding the nanocomposites with different clays, the degradation of PLA upon recycling was also noticeable. However, the reprocessing step caused a better dispersion of the clays in the polymer matrix, leading in this case to improved mechanical, thermal and gas barrier properties in the recycled material.

The decrease of the intrinsic viscosity, and some properties, of recycled PLA might compromise the recyclability of the plastic. Therefore, other objective of this work is the study of different methods to improve the performance of recycled PLA, without significantly increasing the costs of the process. For this, a commercial grade of PLA was 
subjected to a recycling process including a melt processing step, an accelerated aging, a washing step and a second melt reprocessing step. It is in this second processing step in which different additives (such dicumyl peroxide and a chain extender), or different clays, are used to improve the performance of recycled PLA.

The results indicate that both the addition of the peroxide and the chain extender, and the addition of the different clays, led to increased intrinsic viscosity, along with better mechanical and thermal properties of recycled PLA.

Summarizing, mechanical recycling causes the degradation of PLA, and a decrease on its performance. This decrease is relatively small, and the recycled material could be used, even in demanding applications such as packaging. Moreover, there are cost-effective ways for obtaining mechanically recycled PLA with improved properties, which should improve the recyclability of this material. 


\section{PUBLICACIONES DERIVADAS}

Parte de los resultados de esta tesis doctoral se encuentran publicados, o en proceso de publicación, en diferentes revistas científicas. A continuación, se listan, en orden cronológico, las publicaciones derivadas a partir de este trabajo:

- Beltrán, F.R, Lorenzo, V., de la Orden, M.U., Martínez Urreaga, J. 2016. Effect of different mechanical recycling processes on the hydrolytic degradation of poly(l-lactic acid). Polym. Degrad. Stab. 133, 339-348.

- Beltrán, F.R., de la Orden, M.U., Lorenzo, V., Pérez, E., Cerrada, M.L., Martínez Urreaga, J. 2016. Water-induced structural changes in poly(lactic acid) and PLLA-clay nanocomposites. Polymer. 107, 211-222.

- Beltrán, F.R., de la Orden, M.U., Lorenzo, V., Martínez Urreaga, J. 2017. Efecto del reciclado mecánico simulado en las propiedades de nanocompuestos poli(ácido láctico) - haloisita destinados a aplicaciones de envasado. 113, 19-28.

- Beltrán, F.R., Lorenzo, V., Acosta, J., de la Orden, M.U., Martínez Urreaga, J. En prensa. Effect of simulated mechanical recycling processes on the structure and properties of poly (lactic acid). J. Environ. Manag. https://doi.org/10.1016/j.jenvman.2017.05.020.

- Beltrán, F.R., Ortega, E., Solvoll, A.M., Lorenzo, V., de la Orden, M.U., Martínez Urreaga, J. 2017. Effects of Aging and Different Mechanical Recycling Processes on the Structure and Properties of Poly(lactic acid)-clay Nanocomposites. J. Polym. Environ. https://doi.org/10.1007/s10924-017-1117-z.

- Beltrán, F.R., Barrio, I., Lorenzo, V., del Río, B., Martínez Urreaga, J., de la Orden, M.U. En revisión. Valorisation of poly(lactic acid) wastes by mechanical recycling: improvement of the properties of the recycled polymer. Waste Manage. Res. 


\section{INTRODUCCIÓN}

\subsection{Estructura de la tesis}

Esta memoria se compone de 8 capítulos en los que se intenta abordar todos los aspectos de la investigación realizada. En el capítulo 1 se presenta una introducción donde se plantea el problema actual del uso creciente y la valorización de residuos, incluyendo el reciclado mecánico, del poli(ácido láctico) o polilactida, en adelante PLA. En este capítulo se realiza una revisión bibliográfica de los trabajos relacionados con este tema y se enumeran los objetivos de esta tesis. El capítulo 2 está conformado por el marco teórico de esta investigación, en el que se presenta el PLA y los nanocomposites basados en este polímero, así como algunas cuestiones fundamentales del reciclado mecánico de bioplásticos. El capítulo 3 está dedicado a la descripción de los materiales y de la metodología seguida en esta investigación. En el capítulo 4 comienza el análisis y discusión de resultados; concretamente, se analiza en primer lugar el efecto de diferentes procesos de reciclado mecánico en la estructura y propiedades del PLA y sus nanocomposites. El capítulo 5 recoge los resultados obtenidos tras estudiar el efecto del reciclado mecánico en la degradación hidrolítica del PLA y sus nanocomposites.

Tras analizar las propiedades del plástico reciclado, en el capítulo 6 se estudian diferentes alternativas para mejorar las prestaciones del PLA reciclado mecánicamente. En el capítulo 7 se exponen las principales conclusiones obtenidas a partir de los resultados de la investigación, así como algunas recomendaciones para posibles estudios futuros. Finalmente, el capítulo 8 recoge las referencias bibliográficas citadas a lo largo de todo el trabajo.

\subsection{Planteamiento del problema y antecedentes}

El sector plástico constituye una de las industrias más importantes en Europa, generando más de 1,5 millones de empleos y $340000 \mathrm{M} €$ en el año 2015. Los plásticos poseen algunas características muy interesantes, como su comodidad, ligereza, seguridad, bajo coste y buenas propiedades estéticas, que han ocasionado un aumento de su utilización en aplicaciones de envasado. De hecho, se estima que el $40 \%$ de la producción global de plásticos es destinada a este tipo de aplicaciones [93,106]. Sin embargo, la mayoría de los plásticos utilizados en la actualidad provienen de combustibles fósiles, por lo que su creciente utilización se traduce en un gran consumo de recursos no renovables, 
cada vez más escasos. Además, una gran parte de estos materiales es inapropiadamente descartada después de su primer uso, lo que genera un vertido incontrolado al entorno de grandes cantidades de residuos difícilmente degradables (se estima que los plásticos representan un $20 \%$ de los desechos sólidos municipales), y por lo tanto un problema medioambiental de escala global $[11,97]$.

Entre las respuestas a los problemas mencionados anteriormente, se puede destacar el desarrollo de plásticos biodegradables y plásticos obtenidos a partir de fuentes renovables, muchos de los cuales son también biodegradables. Todos ellos son los denominados bioplásticos [99].

Entre los bioplásticos, el poli(ácido láctico) (PLA) es uno de los materiales mejor establecidos en el mercado. El PLA es un poliéster alifático, producido principalmente mediante la polimerización por apertura de anillo de la lactida, el dímero cíclico del ácido láctico. El ácido láctico se obtiene a partir de la fermentación de los azúcares presentes en productos como el maíz o la patata $[8,105]$. El PLA presenta muy buenas prestaciones, de hecho, algunas propiedades son comparables con las de polímeros convencionales como el poliestireno y el polietilentereftalato (PET). Por ejemplo, el PLA es considerado un polímero con buenas propiedades mecánicas, con un módulo elástico variando entre 3000 y $4000 \mathrm{MPa}$, y una resistencia a la tracción que ronda los $60 \mathrm{MPa}$. El PLA también presenta propiedades de barrera frente a gases aceptables, superando incluso al poliestireno en algunos casos [8,9]. Otro de los aspectos más llamativos de este polímero es su biocompatibilidad, y el hecho haber sido aprobado por la FDA para estar con contacto con alimentos. Además de estas propiedades, es importante destacar que el PLA tiene unos costes de producción moderados y que posee una buena procesabilidad, pudiendo ser procesado mediante extrusión, moldeo por inyección, soplado, termoconformado e hilado [47].

Estas características, junto con la gran variedad de grados disponibles comercialmente, han ampliado el mercado de aplicaciones del PLA, que ha pasado de ser un material empleado principalmente en aplicaciones biomédicas (fabricación de implantes y suturas entre otros) a ser un material utilizado en un amplio rango de aplicaciones.

Por ejemplo, la industria automotriz ha planteado la utilización del PLA en la fabricación de algunas partes del interior del coche. Sin embargo, para su total aplicabilidad en este campo se deben resolver algunos problemas relacionados con la baja estabilidad térmica y la susceptibilidad del PLA frente a la degradación hidrolítica y termomecánica [21,29]. Otro sector que está apostando fuertemente por la utilización del PLA como sustituto de otros plásticos basados en combustibles fósiles, es el sector del envasado de alimentos. Compañías como Danone (Alemania), Wal-Mart (Estados Unidos), PURALACT (Holanda), Polenghi LAS (Italia), NobleJuice (Estados Unidos), Auchan (Francia), entre otras utilizan envases fabricados parcial o totalmente con PLA. De hecho, la mayor parte de la producción global de PLA es empleada en la fabricación de envases, especialmente aquellos destinados a contener productos frescos [2,29]. 
La expansión de las aplicaciones comerciales del PLA ha traído como consecuencia un crecimiento sostenido de la producción de este bioplástico en los últimos años. Entre el año 2012 y el año 2014 la producción de PLA pasó de 180000 a 205000 t. Además, se prevé un crecimiento anual del 10\% entre 2016 y 2021 [2].

Si bien es cierto que la producción del PLA consume una menor cantidad de recursos y energía, y posee un menor impacto medioambiental que la de los polímeros basados en combustibles fósiles, la utilización masiva de este bioplástico podría traer consigo diferentes problemas, tanto sociales como medioambientales. En primer lugar, la gran superficie de tierra necesaria para cultivar las materias primas utilizadas en la producción del PLA podría llevar a algunos agricultores a cambiar la producción de alimentos, con menor rendimiento económico, por los cultivos utilizados en la manufactura del PLA. Esta situación amenazaría gravemente la sostenibilidad y el bienestar de los países en vías de desarrollo, y aumentaría de forma importante el impacto medioambiental de la producción del PLA [65,77]. En segundo lugar, hay que tener en cuenta el dilema moral de utilizar potenciales recursos alimentarios para la fabricación de plásticos, especialmente con los problemas de hambruna que experimentan algunos países en la actualidad [111]. Finalmente, es necesario considerar la baja velocidad de degradación de los grados de PLA usados en la fabricación de envases o fibras, que son muy diferentes de los grados empleados en aplicaciones biomédicas [47]. La baja velocidad de degradación del PLA podría ocasionar la saturación de las instalaciones de compostaje, y generar una acumulación importante de residuos, sobre todo si se masifica la utilización de este bioplástico [12,81,101].

Estos problemas han llamado la atención tanto de la industria como de la comunidad científica, por lo que se está trabajando en el desarrollo de métodos que permitan la reducción y valorización de los residuos de PLA. Entre las alternativas disponibles se encuentran el compostaje, la valorización energética, el reciclado químico y el reciclado mecánico [29].

El compostaje podría ser una alternativa interesante para la valorización de residuos contaminados de PLA, ya que este polímero es compostable en condiciones industriales. Sin embargo, esta alternativa presenta algunos inconvenientes. En primer lugar se encuentra la baja velocidad de degradación de los grados comerciales de PLA; en segundo lugar, muchas instalaciones de compostaje no aceptan bioplásticos, debido a los problemas de control de calidad que estos podrían generar; finalmente, el compostaje no implica una disminución en el consumo de materias primas, ya que el PLA valorizado por este método debe ser sustituido por PLA nuevo [29,39].

La valorización energética consiste en la producción de energía a partir de los plásticos, lo que contribuye a la reducción de la dependencia en los combustibles fósiles. Sin embargo, al igual que en el caso del compostaje, este método no disminuye el uso de las materias primas utilizadas en la fabricación del PLA. Además, algunos residuos pueden estar contaminados, por lo que hay que ser cuidadoso para evitar la liberación de compuestos perjudiciales para el medio ambiente y la salud [11]. 
El reciclado químico se basa en la despolimerización del PLA, bien por la vía hidrolítica (para producir ácido láctico) o bien por vía térmica (para dar lugar a lactida). Estos compuestos podrían ser usados en la producción de PLA, ayudando a reducir el consumo de las materias primas necesarias para la manufactura de este material. Sin embargo, este proceso se encuentra poco estudiado y, debido a las elevadas purezas requeridas, actualmente no es rentable en comparación con la obtención de monómeros mediante la fermentación [108].

Finalmente, el reciclado mecánico consiste en la recuperación, clasificación, triturado y reprocesado de los desechos de PLA. De acuerdo con diferentes autores, representa la alternativa para la valorización de los residuos de PLA con más bajo impacto medioambiental. Por ejemplo, Piemonte [91] empleó la metodología de análisis de ciclo de vida (ACV) para estudiar diferentes alternativas de valorización, entre las que se encontraban el reciclado mecánico, el compostaje, la valorización energética y la digestión anaeróbica. En dicho estudio se llega a la conclusión de que el reciclado mecánico es la alternativa más interesante desde el punto de vista medioambiental, permitiendo un ahorro de energía y materias primas en comparación con las otras alternativas estudiadas. En un estudio similar, realizado por Rossi et al. [101], se aplicó la metodología ACV a diferentes alternativas para la valorización de residuos de PLA y almidón termoplástico (TPS), tales como reciclado mecánico, compostaje industrial, digestión anaeróbica y valorización energética. Al igual que en el estudio realizado por Piemonte, Rossi et al. concluyen que el reciclado mecánico es la alternativa que presenta el menor impacto medioambiental y permite un mayor aprovechamiento de los residuos. Rossi et al. reportan que el compostaje industrial es la alternativa con peor rendimiento, debido al bajo contenido de nitrógeno, potasio y fósforo del PLA, lo que limitaría su uso como fertilizante. De manera similar, Cosate de Andrade et al. [39] comparan mediante ACV el impacto del reciclado mecánico, reciclado químico y compostaje. En este trabajo llegan a la conclusión de que el impacto medioambiental del reciclado mecánico es menor que el de las otras alternativas estudiadas.

Si se consideran los resultados de los estudios mencionados anteriormente, se puede observar que el reciclado mecánico podría representar a priori una alternativa muy interesante para la reducción y valorización de los residuos de PLA, especialmente aquellos provenientes de aplicaciones de envasado, permitiendo reducir el consumo de energía y materias primas, al mismo tiempo que se generaría un importante número de empleos. Sin embargo, la evaluación de la viabilidad del reciclado mecánico de PLA requiere de un enfoque global, que tome en cuenta los diferentes factores que influyen en el proceso.

Por una parte, es necesario desarrollar sistemas de recogida viables para el PLA. En este proceso intervienen una serie de factores cruciales, que se enumeran a continuación $[29,38,81]$ :

- Garantizar la disponibilidad de capital monetario para adquirir equipos y operar las instalaciones. Dichos recursos podrían ser obtenidos de los fabricantes de bioplásticos. 
- Mejorar la identificación del material, mediante el establecimiento de un nuevo código de identificación de plástico exclusivo para el PLA. En la actualidad comparte su categoría con otros plásticos (7 - Otros).

- Considerar la posible contaminación de otros plásticos presentes en la corriente de reciclado, como el PET, que ha sido investigado por diferentes autores [62,71].

- Contar con un consumo del polímero virgen de al menos 200000 t al año, con la finalidad de asegurar la rentabilidad del reciclado mecánico. Es importante destacar que en el caso del PLA este umbral se superó en el año 2014, cuando se alcanzó una producción global de 205000 t [2].

Por otra parte, también es muy importante estudiar el efecto del reciclado mecánico en las prestaciones del PLA. Si las propiedades del material reciclado son muy pobres, sería muy complicado obtener productos con valor añadido, por lo que el reciclado mecánico sería inviable y se vería amenazado el bajo impacto medioambiental del PLA [81,108].

A la hora de considerar el reciclado mecánico, se pueden considerar dos escenarios deferentes. Si el residuo de PLA procede del propio proceso de fabricación y no se encuentra contaminado por otros plásticos, restos de alimentos, etc., hablamos de un residuo postindustrial. Se trata de un material de gran valor, muy homogéneo, no contaminado y muy escasamente degradado. En cambio, si se trata de un residuo de postconsumo, recogido tras haber sido consumido, se trata de un material de mucho menor valor, muy poco homogéneo, que puede estar degradado y contaminado de forma variable y en algunos casos muy importante.

El reciclado mecánico del residuo postindustrial es sencillo, ya que no requiere etapas de separación o lavado, sino sólo etapas de reprocesado, y ha sido ampliamente estudiado. Diversos, autores han realizado estudios para determinar el efecto de múltiples reprocesados en las prestaciones del PLA. Por ejemplo, Pillin et al. [92] estudiaron el efecto de varios ciclos de inyección en las propiedades térmicas, mecánicas y reológicas del PLA, encontrando una disminución de más del $60 \%$ en el peso molecular promedio, $M_{w}$, después de 7 ciclos de inyección. Esta disminución fue relacionada con la presencia de cadenas poliméricas más cortas, como consecuencia de la degradación termomecánica del PLA. La degradación del PLA también se vio reflejada en sus propiedades térmicas, donde se observó una disminución de $10^{\circ} \mathrm{C}$ en la $T_{g}$, un incremento en la habilidad para cristalizar y una disminución de la temperatura de fusión del polímero. Las propiedades mecánicas y reológicas también se vieron afectadas, apreciándose una disminución de la resistencia a la tracción de 66 a 25 MPa después de 7 ciclos de inyección, así como una disminución en la viscosidad del PLA, que pasó de 3960 a 25 Pa·s. Pillin et al. también reportan que la adición de quinona durante el reprocesado ayuda a disminuir la degradación del polímero, y por tanto limita el efecto del reprocesado en las propiedades del PLA.

Żenkiewicz et al. [117] determinaron el efecto de hasta 10 ciclos de extrusión en el índice de fluidez y en las propiedades térmicas, mecánicas y de barrera del PLA, reportando un incremento continuado del índice de fluidez con cada ciclo de extrusión, 
lo que indica la degradación del PLA durante el reprocesado. Esta degradación también fue observada mediante calorimetría diferencial de barrido, DSC, donde se encontró un descenso de la temperatura de cristalización en frío, $T_{c c}$. En cuanto a las propiedades mecánicas y de barrera, ambas empeoraron durante el reprocesado. Es especialmente llamativo el incremento de la permeabilidad del vapor de agua, que alcanzó valores $39 \%$ más altos después de 10 ciclos de extrusión.

Nascimento et al. [80] discutieron el efecto de un ciclo de extrusión, seguido de un ciclo de inyección, en las propiedades térmicas y mecánicas del PLA. En este trabajo se reporta un incremento del índice de fluidez del PLA, lo que indica la degradación del material después de un ciclo de reprocesado. Esta degradación ocasionó un incremento en la habilidad para cristalizar del PLA, sin embargo, las variaciones de la resistencia a la tracción y el módulo elástico fueron muy pequeñas.

Badía et al. [14] estudiaron los cambios estructurales en el PLA como consecuencia de hasta 5 ciclos de reprocesado, y su efecto en las propiedades mecánicas y térmicas del plástico. Los resultados obtenidos señalaron que la estructura química del PLA no se ve afectada por el reprocesado; sin embargo, si se observó una reducción considerable del peso molecular promedio viscosimétrico, $M_{v}$, alrededor del $50 \%$, después de 5 ciclos de reprocesado. En la misma línea que Pillin et al., en este trabajo se reporta un incremento en la habilidad para cristalizar del PLA reprocesado, y una disminución del módulo elástico y de la resistencia a la tracción. En otro estudio, Badía et al. [13] analizaron el efecto de 3 ciclos de reprocesado mecánico en la cinética de absorción de agua y en la degradación hidrolítica del PLA a temperaturas por encima de $T_{g}$, encontrando una disminución del coeficiente de difusión del agua a medida que aumentaban en número de ciclos de reprocesado. También reportaron una mayor cristalinidad en los materiales reciclados después de la degradación hidrolítica, atribuyendo este comportamiento a la presencia de cadenas más cortas con una mayor movilidad, lo que les permite reorganizarse en estructuras cristalinas con una mayor facilidad.

Hopmann et al. [54] se centran en estudiar el efecto de distintos porcentajes de PLA reprocesado en el peso molecular y propiedades mecánicas y ópticas del material reciclado. Este estudio concluye que la utilización de hasta 50\% de PLA reprocesado tiene un efecto limitado en la viscosidad y peso molecular del material, por lo que no debería afectar la procesabilidad de las mezclas de material virgen y reprocesado. En cuanto a las propiedades mecánicas, se reporta un incremento de la fragilidad del material durante el reprocesado. Finalmente, se encontró una variación pequeña, pero perceptible en el color de los filmes, adquiriendo estos una tonalidad amarillenta al incrementar la proporción de material reprocesado.

Brüster et al. [23] analizaron el efecto de varios ciclos de reprocesado en el peso molecular y las propiedades térmicas y mecánicas del PLA con y sin plastificante. Se observó una disminución de más de $45 \%$ de $M_{w}$ del PLA sin plastificar después de 5 ciclos de reprocesado. En el caso del material plastificado, no fue posible cuantificar el descenso del peso molecular debido a la presencia de entrecruzamientos. En cuanto a las 
propiedades mecánicas, en ambos casos se observa una disminución de la resistencia a la tracción después de 3 y 5 ciclos de reprocesado. Así mismo, se observó que la tenacidad de ambos materiales disminuye durante el reprocesado; sin embargo, esta variación es mayor para el material plastificado (55\% del PLA plastificado y 33\% del PLA puro). Se observó también un aumento de la habilidad para cristalizar en el PLA plastificado, debido a la presencia de cadenas más cortas como consecuencia de la degradación. Al igual que lo reportado en las medidas de $M_{w}$, la presencia de entrecruzamientos impidió observar este comportamiento en el material plastificado.

Más recientemente, Tuna et al. [111] estudiaron el efecto de dos extensores de cadenas, concretamente Joncryl ADR-4368 y 1,4-fenilen diisocianato, en las propiedades reológicas, mecánicas, térmicas y morfológicas de un PLA sometido a un proceso de extrusión, seguido de un segundo reprocesado que consistió en un ciclo de extrusión y un ciclo de inyección. Las medidas de viscosidad intrínseca mostraron la degradación del PLA durante el reprocesado, así como un incremento de la viscosidad al añadir cualquiera de los dos extensores de cadena. En cuanto a las propiedades de los materiales, por una parte, se encontró que la resistencia a la tracción disminuye en el reprocesado, como consecuencia de la degradación, pero aumenta en los materiales con los extensores de cadena. Por otra parte, no se hallaron variaciones significativas en el comportamiento térmico de ninguna de las muestras.

Como se puede observar, los estudios citados anteriormente consideran sólo el reciclado postindustrial, por lo que se centran sólo en la degradación durante el procesado y el reprocesado del PLA. Estos trabajos no consideran el reciclado de postconsumo, dejando de lado el deterioro que podría sufrir el material durante su vida útil. Esta degradación, que puede ser térmica, fotoquímica o hidrolítica podría ocasionar un importante descenso en el peso molecular y la viscosidad, así como afectar negativamente a la procesabilidad y a las prestaciones del material reciclado.

Son muy pocos los estudios publicados hasta la fecha que consideran el reciclado mecánico de residuos de PLA de postconsumo. Por ejemplo, Chariyachotilert et al. [32] estudiaron el efecto de una etapa de lavado en el reciclado de PLA de post-consumo, y en las propiedades de diferentes mezclas de PLA reciclado y virgen. Los resultados obtenidos apuntan a que la presencia de $40 \%$ de material reciclado no modifica de manera importante el peso molecular, la estructura química o las propiedades térmicas y mecánicas del PLA. Sin embargo, si se observó un ligero oscurecimiento de las muestras al aumentar el contenido de material reciclado.

Yarahmadi et al. [114] utilizaron un protocolo de envejecimiento acelerado de 7 semanas, a $50{ }^{\circ} \mathrm{C}$ y con una humedad relativa del $90 \%$, para simular la degradación durante la vida útil del PLA y sus mezclas con otras resinas como polietileno o policarbonato. El reprocesado múltiple del PLA, hasta 6 ciclos, causó un incremento del $40 \%$ en el índice de fluidez del PLA puro, indicando la degradación del PLA. Sin embargo, la resistencia al impacto o la Tg del material no se vieron afectadas. En cuanto a las mezclas, el reprocesado no afectó de forma importante el módulo elástico, aunque si se observaron variaciones en el alargamiento a la rotura. La adición de la etapa de 
envejecimiento acelerado causó una degradación importante tanto en el PLA, como en las mezclas, lo que repercutió en las propiedades mecánicas de los materiales.

A pesar de la existencia de unos pocos trabajos relativos al reciclado mecánico de residuos de postconsumo, hasta la fecha no existe un trabajo sistemático sobre la influencia de cada una de las etapas que atraviesa un residuo de postconsumo sometido a reciclado mecánico sobre la viscosidad y las propiedades del reciclado. Por ejemplo, cabe esperar que los residuos de plásticos empleados en envasado de alimentos tengan que ser sometidos a un proceso de lavado enérgico antes de ser reprocesados. Dependiendo de las condiciones, ese lavado podría causar una importante degradación hidrolítica en el PLA, que es un poliéster alifático. Tampoco se ha estudiado de forma sistemática el efecto de los diferentes tipos de degradación (térmica, fotoquímica, hidrolítica) que puede sufrir el plástico durante su vida útil.

Si se toman en cuenta los resultados de los estudios citados anteriormente, sí se puede concluir que el reprocesado mecánico conduce a una disminución del peso molecular y de las prestaciones del PLA, lo que podría comprometer su reciclabilidad y su bajo impacto medioambiental. Por ello, en los últimos años se ha estudiado la utilización de algunos métodos que permiten aumentar el peso molecular (parámetro que es muy importante de cara al procesado del material reciclado, ya que determina la viscosidad) y mejorar las propiedades del PLA de bajo $M_{w}$. Entre los métodos conocidos se pueden resaltar: tratamientos térmicos, extrusión reactiva, utilización de refuerzos y la mezcla con otros materiales [12].

Los tratamientos térmicos se centran en incrementar la cristalinidad del PLA sometiéndolo a temperaturas mayores que $T_{g}$, pero menores que la temperatura de fusión. Los trabajos de Carrasco et al. [27] y Nascimento et al. [80] indican que el aumento de la cristalinidad va ligado a una mejora del módulo de Young y de la resistencia a la fluencia. Sin embargo, este aumento de la cristalinidad causa un aumento en la fragilidad del material, así como un descenso en la transmisión de luz.

La extrusión reactiva consiste en la utilización de diferentes aditivos durante el procesado del PLA, con la finalidad de lograr entrecruzamientos de cadenas, aumentar su peso molecular y viscosidad, y mejorar por tanto sus prestaciones. El uso de aditivos para aumentar el peso molecular del PLA ha sido estudiado durante los últimos años, Por ejemplo, Meng et al. [74] reportaron un gran incremento de la viscosidad compleja del PLA y sus nanocomposites con arcilla al añadir diferentes extensores de cadena, concretamente dianhidrido piromelítico, hexametilendiisocianato y un extensor con grupos epoxi, el Joncryl ADR-4368. En la misma línea, el estudio realizado por Al Itry et al. [3], utilizando Joncryl ADR-4368 y PLA, reporta un incremento de hasta $80 \%$ del peso molecular del polímero. Jaszkiewicz et al. [56] también reportan un aumento del peso molecular, y una ligera ramificación del PLA al utilizar pequeñas cantidades de un extensor de cadena comercial, concretamente, CESA-Extend, que se basa en PLA y un reactivo con grupos epoxi. Finalmente, en otro estudio se utiliza el trifenilfosfito como aditivo para incrementar el peso molecular del PLA, obteniendo incrementos de más del $50 \%$ al añadir $2 \%$ de trifenilfosfito [75]. 
El uso de diferentes partículas, como las nanoarcillas, para mejorar las propiedades mecánicas y de barrera del PLA ha sido ampliamente estudiado durante los últimos años [97]. Algunos autores como Araujo et al. [5], Molinaro et al. [76] y Gorrasi et al. [51] señalan que la adición de pequeñas cantidades de montmorillonita o haloisita conduce a una reducción de la permeabilidad, así como a un aumento de la estabilidad térmica y del módulo de Young.

La utilización del PLA reciclado en mezclas con otros polímeros podría parecer la opción más interesante desde el punto de vista de costes. Sin embargo, es importante obtener una buena miscibilidad entre los componentes de la mezcla; de otra forma, las propiedades del material resultante serían muy pobres. En este sentido, los estudios realizados por Chariyachotilert et al, con PLA de post-consumo y PLA virgen, y Hopmann et al., con PLA reprocesado y PLA virgen, señalan que un material con un contenido de PLA virgen de $40 \%$ o más presenta propiedades mecánicas, ópticas y térmicas comparables a las de un material $100 \%$ virgen.

\subsection{Enfoque de la tesis y objetivos}

Esta tesis se centra en el estudio de los efectos de la degradación en uso y de diferentes procesos de reciclado mecánico en la estructura y en algunas propiedades del PLA, consideradas claves en las aplicaciones de envasado de alimentos. El estudio se refiere a residuos de postconsumo, por lo que se emplearon materiales sometidos a diferentes procesos de degradación y lavado antes de su reprocesado mecánico. Dado el creciente uso de nanocomposites de PLA, en el estudio se incluye también el reciclado de nanocomposites de PLA con diferentes arcillas. Entre las propiedades estudiadas se encuentran la estabilidad térmica, la resistencia a la degradación hidrolítica y las propiedades ópticas, mecánicas y de barrera frente a diferentes gases. Así mismo, se analizan también diferentes alternativas para obtener un PLA reciclado mecánicamente con propiedades mejoradas, sin incrementar de forma significativa los costes del proceso. Con este trabajo se pretende profundizar en el conocimiento del reciclado mecánico de bioplásticos, concretamente del PLA, con la finalidad de mejorar su reciclabilidad y, por tanto, incrementar su interés como alternativa a los plásticos convencionales.

Para llevar a cabo este trabajo se simularon, a escala de laboratorio, diferentes procesos de reciclado mecánico de PLA, incluyendo varias etapas de envejecimiento acelerado, lavado y reprocesado. Los materiales obtenidos fueron caracterizados mediante diferentes técnicas experimentales, que incluyen: viscosimetría, espectroscopía infrarroja, microscopía electrónica, difracción de rayos $\mathrm{X}$, calorimetría diferencial de barrido, análisis termogravimétrico, medidas de microdureza, espectroscopia UV-Visible y medidas de permeabilidad de gases. Finalmente, los materiales reciclados fueron sometidos a diferentes procesos, como la adición de arcillas y la adición de extensores de cadena, para incrementar su viscosidad intrínseca y/o mejorar sus propiedades finales. 
De acuerdo con el enfoque presentado anteriormente, los objetivos principales de este trabajo son:

- Estudio de la viabilidad del reciclado mecánico de PLA dedicado a aplicaciones de envasado, simulando las condiciones del material en su ciclo de vida y durante su reprocesado mecánico y analizando el efecto que el reciclado pueda tener sobre las propiedades del plástico reciclado.

- Análisis de diferentes alternativas para mejorar las prestaciones del PLA reciclado mecánicamente, sin aumentar de manera significativa los costes del proceso.

Estos objetivos principales pueden, a su vez, dividirse en varios objetivos secundarios que se enumeran a continuación:

- Someter un PLA virgen, y sus nanocomposites con las arcillas haloisita y montmorillonita, a diferentes procesos de reciclado, incluyendo etapas de envejecimiento fotoquímico, térmico e hidrotérmico, así como una etapa de lavado, previamente al reprocesado mediante extrusión del polímero.

- Medir la viscosidad intrínseca de los materiales reciclados, y compararlos con los de los materiales vírgenes.

- Estudiar los cambios morfológicos y estructurales en los materiales como consecuencia de los diferentes procesos de reciclado.

- Analizar el efecto de los diferentes procesos de reciclado en las propiedades térmicas, ópticas, mecánicas y de barrera frente a gases del PLA.

- Comparar la cinética de absorción de agua, a dos temperaturas diferentes, del PLA y sus nanocomposites vírgenes y reciclados.

- Estudiar la resistencia frente a la degradación hidrolítica, a dos temperaturas diferentes, de los materiales reciclados y compararla con la de los materiales vírgenes.

- Obtener nanocomposites con arcillas modificadas a partir de la matriz polimérica reciclada.

- Comparar la viscosidad intrínseca, la estructura y las propiedades térmicas, ópticas, mecánicas y de barrera frente a gases de los nanocomposites de matriz reciclada con los del PLA virgen y el PLA reciclado.

- Obtener materiales reciclados con diferentes extensores de cadenas y/o agentes de entrecruzamiento.

- Comparar la viscosidad intrínseca, la estructura y las propiedades térmicas, ópticas, mecánicas y de barrera frente a gases de materiales reciclados con extensores de cadenas y/o agentes de entrecruzamiento con los del PLA virgen y el PLA reciclado. 


\section{MARCO TEÓRICO}

\subsection{Poli(ácido láctico)}

El poliácido láctico, o PLA, es un poliéster alifático, derivado del ácido 2hidroxipropiónico, o ácido láctico. El PLA, cuya estructura se muestra en la Fig. 1, es un termoplástico generalmente considerado biodegradable y compostable (en condiciones industriales). En los últimos años ha atraído un gran interés debido a sus buenas propiedades mecánicas y ópticas, su biocompatibilidad, así como a su procesabilidad empleando tecnología actualmente disponible. Además de sus buenas propiedades, la estructura estereoquímica del PLA puede ser fácilmente controlada, permitiendo obtener diferentes grados comerciales con diferentes propiedades [7,50].<smiles>[R]C(C)(C)C(=O)C(C)OC([2H])([2H])C</smiles>

Fig. 1. Estructura química del PLA.

\subsubsection{Producción industrial del PLA}

Existen varios métodos para la obtención de PLA de alto peso molecular, que se muestran en la Fig. 2: (i) polimerización por condensación directa; (ii) condensación con deshidratación azeotrópica y (iii) polimerización mediante apertura de anillo de lactida. Esta última es, con diferencia, el método más utilizado a nivel industrial para la obtención de PLA de alto peso molecular. Esto se debe a las dificultades que presentan los otros métodos. La condensación directa es la ruta más económica, sin embargo, conduce a la obtención de un polímero de bajo peso molecular. Para corregir esta desventaja se emplean extensores de cadena, los cuales aumentan el coste y la complejidad del proceso. La condensación con destilación azeotrópica es un proceso que puede conducir a la obtención de PLA de alto peso molecular, pero emplea grandes cantidades de catalizador, 
por lo que es necesario un proceso de purificación del PLA, para evitar los residuos que podrían ser perjudiciales para algunas aplicaciones $[8,10]$.

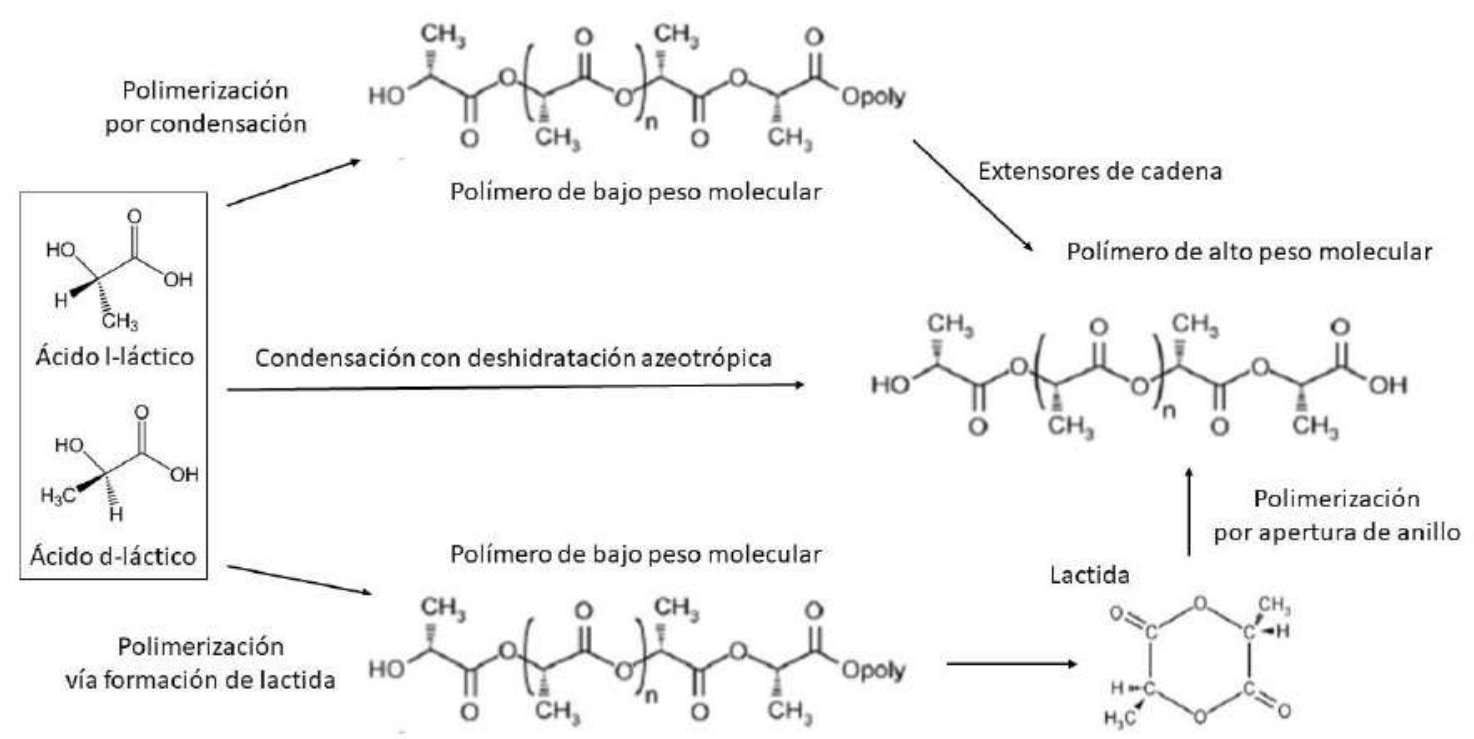

Fig. 2. Métodos para la obtención de PLA de alto peso molecular. Adaptado de [10]

La polimerización mediante apertura de anillo de lactida es el método patentado por Cargill Dow LLC, y es utilizado por NatureWorks, el principal productor de PLA. Este proceso comienza con la obtención de ácido láctico, que es una molécula quiral que se encuentra en dos formas, ácido l-láctico y ácido d-láctico, mediante la fermentación de los azúcares presentes en diferentes productos naturales como el maíz, la patata o la remolacha. El ácido láctico obtenido es polimerizado mediante condensación, dando lugar a un PLA de bajo peso molecular. Este polímero de bajo peso molecular, o prepolímero, es convertido mediante transesterificación interna en una mezcla de estereoisómeros de lactida, el dímero cíclico del ácido láctico. Durante la transesterificación se puede obtener los diferentes estereoisómeros de la lactida: 1-lactida, d-lactida y dl-lactida. La lactida es posteriormente purificada y polimerizada mediante apertura de anillo, obteniéndose un PLA de alto peso molecular y de alta pureza óptica. Dicha polimerización puede ser realizada en fundido, y emplea catalizadores como el octoato de estaño [8,78].

\subsubsection{Estructura del PLA}

Como se ha mencionado anteriormente, tanto el ácido láctico como la lactida son compuestos quirales que presentan distintos estereoisómeros. La naturaleza quiral de estos compuestos se traduce también en la posibilidad de obtener diferentes tipos de PLA, concretamente el PLLA, PDLA y PDLLA, los cuales poseen diferentes propiedades fisicoquímicas y mecánicas. Las polilactidas ópticamente puras son materiales cristalinos, con un punto de fusión que supera los $200^{\circ} \mathrm{C}$. Sin embargo, a nivel comercial se obtiene 
PLLA con diferentes contenidos de isómero d. La presencia de d-lactida produce imperfecciones en la estructura regular del PLLA, lo que produce un descenso en la temperatura de fusión, y la obtención de un material semicristalino (menos de $20 \%$ de dlactida) o completamente amorfo (más del $20 \%$ de d-lactida). Si se controlan las condiciones de la polimerización, se pueden obtener una amplia gama de grados de PLA con diferentes propiedades, lo que ha ayudado a la implementación de este plástico en diferentes aplicaciones. Cabe destacar que la mezcla 1:1 de PLLA y PDLA forma un estereocomplejo equimolar, con prestaciones superiores a la de los estereoisómeros puros (se pueden alcanzar temperaturas de fusión de hasta $230^{\circ} \mathrm{C}$ ) $[10,50,99]$.

Otro factor que puede influir en las prestaciones del PLA es la estructura cristalina del polímero. Según las condiciones de preparación empleadas, el PLA puede presentar hasta 4 formas cristalinas (o polimorfos) diferentes, denominados $\alpha, \alpha^{\prime}, \beta$ y $\gamma$, siendo la primera la forma cristalina más común. Esta forma $\alpha$ consta de dos cadenas poliméricas en una conformación helicoidal $10_{3}$, empacadas en una celda unitaria ortorrómbica [35,58]. La forma $\beta$ consiste en el empaquetamiento frustrado de tres hélices triples en una celda unitaria trigonal, y se obtiene a altas temperaturas y altas relaciones de estirado [95]. La forma $\gamma$ se obtiene mediante la cristalización epitaxial en un sustrato de hexametilbenceno, y está formada por dos hélices antiparalelas, empacadas en una celda unitaria ortorrómbica [28]. Finalmente, la forma $\alpha^{\prime}$ fue observada recientemente por Zhang et al. [118] mediante espectroscopía FTIR y XRD, al estudiar las estructuras cristalinas formadas durante el tratamiento térmico de PLLA a diferentes temperaturas. Esta forma cristalina consiste en una estructura muy similar a la forma $\alpha$, pero con un empaquetamiento menos ordenado, resultado de la presencia de interacciones más débiles entre cadenas que en la forma $\alpha$.

Otros estudios señalan que la proporción entre los cristales $\alpha^{\prime} / \alpha$ depende en gran parte de la temperatura a la que se realice la cristalización: la forma $\alpha$ ' se obtiene por debajo de $100{ }^{\circ} \mathrm{C}$, la forma $\alpha$ por encima de $120^{\circ} \mathrm{C}$, y una mezcla de ambas se obtiene entre 100 y $120^{\circ} \mathrm{C}$. Además, algunos estudios señalan que la transformación de la forma $\alpha$ ' en $\alpha$ tiene lugar al calentar el material hasta su punto de fusión, y solo comprende pequeños reordenamientos dentro de la celda unitaria $[58,84,85]$. La presencia de estas dos formas cristalinas, $\alpha$ ' y $\alpha$, pueden afectar las propiedades del PLA. Por ejemplo, Cocca et al. [35] y Kalish et al. [57] han reportado que el PLA con mayor proporción de cristales $\alpha$ presenta mejores propiedades de barrera frente al vapor de agua, mayor módulo de Young y mayor estabilidad térmica.

\subsubsection{Aplicaciones del PLA}

La producción de PLA ha aumentado en los últimos años debido a su coste relativamente bajo, y a la buena percepción del impacto medioambiental de este polímero, lo que ha abierto mercados que antes se encontraban restringidos a polímeros derivados del petróleo. El PLA se emplea actualmente en un amplio rango de aplicaciones, que incluye la fabricación de envases y cubertería desechables, coches y algunos productos médicos [29]. 
En un principio, el PLA se utilizaba principalmente en aplicaciones biomédicas, tales como estents, suturas quirúrgicas, fijaciones para reparación de lesiones óseas, implantes y dispositivos de administración de fármacos. La importancia del PLA en este mercado se debe a su biocompatibilidad y biodegradabilidad [29,47]. Sin embargo, las mejoras en las propiedades y la disminución de los costes de producción han abierto una otros mercados para el PLA.

Por ejemplo, la buena procesabilidad en fibras del PLA, junto con su capilaridad, han fomentado las aplicaciones textiles de este polímero. Se han desarrollado toallitas, filtros de té, filtros de agua, entre otras cosas. Además, las fibras de PLA han despertado interés en la industria automotriz y textil. Sin embargo, existen algunas limitaciones relacionadas con la baja resistencia térmica y a la abrasión, por lo que se deben obtener grados con mejores prestaciones para poder competir con materiales tradicionales como el PET o el algodón [21,29].

El uso de PLA en aplicaciones agrícolas ha aumentado su atractivo debido a los problemas en la gestión de residuos ocasionados por el uso masivo de plásticos derivados de combustibles fósiles, concretamente el polietileno. Este mercado se encuentra aún en las primeras etapas de desarrollo, en las que la mayoría de las pruebas se hacen a escala de laboratorio por el coste relativamente alto del material. Hasta ahora, el uso de homopolímeros de PLA en aplicaciones agrícolas se ha visto limitado por la fragilidad del polímero, y por su baja biodegradabilidad en condiciones normales. Por ello se trabaja actualmente en el desarrollo de mezclas de PLA con otros poliésteres biodegradables o plastificantes para la producción de materiales agrícolas basados en PLA [8,29].

Pese a las diversas aplicaciones citadas PLA, en la actualidad la mayor parte de la producción de este bioplástico se destina a aplicaciones de envasado, especialmente de alimentos. Esto, a pesar de que presenta algunas desventajas, como sus limitadas propiedades de barrera frente a gases. Sin embargo, las prestaciones del PLA han sido mejoradas mediante diferentes métodos, como el procesado a medida, la mezcla con otros polímeros o el uso de diferentes aditivos (nucleantes, antioxidantes y plastificantes). Por ejemplo, se han empleado filmes orientados de PLA como envases de patatas y bollería. Los filmes no orientados son utilizados en el termoconformado de envases para productos frescos como fresas, cebollas, ensaladas, yogures, entre otros. El PLA también puede ser empleado en la fabricación de filmes y etiquetas retractiles, sin embargo, para mejorar las prestaciones de estos materiales se suele mezclar el PLA con otros poliésteres. En los últimos años también ha aumentado el interés por la manufactura de botellas de PLA para zumos y agua. Sin embargo, este mercado se encuentra limitado solo a bebidas no carbonatadas, debido a la alta permeabilidad del PLA al $\mathrm{CO}_{2}$. Por lo tanto, es necesario mejorar las prestaciones del PLA para poder competir con polímeros como el PET. Por último, la utilización de PLA para envases de microondas y vasos desechables se encuentra mayormente limitada por la baja temperatura de deflexión térmica del PLA (entre 55 y $65^{\circ} \mathrm{C}$ ). Sin embargo, algunas alternativas como el uso de agentes nucleantes, la manipulación de la estereoquímica y las mezclas con otros polímeros puede impulsar su uso en aplicaciones donde se requiera una mayor resistencia térmica $[8,10,47]$. 


\subsection{Nanocomposites basados en PLA}

Como se ha mencionado anteriormente, el PLA posee unas propiedades muy interesantes, un alto módulo de Young, calidad óptica, biocompatibilidad, biodegradabilidad y procesabilidad. Sin embargo, tiene como desventaja su baja flexibilidad, resistencia al impacto, temperatura de distorsión térmica y lenta cristalización. Estos problemas pueden limitar el uso del PLA en aplicaciones específicas muy exigentes, por lo que en los últimos años se han estudiado diferentes alternativas para mejorar las prestaciones del PLA, entre las que se encuentra la dispersión de refuerzos de escala nanométrica en la matriz polimérica, dando lugar a los nanocomposites [78,97,99].

Un nanocomposite polimérico es un material multifásico, en el que al menos una de las fases, normalmente el refuerzo, posee una o más dimensiones por debajo de los $100 \mathrm{~nm}$. Estas pequeñas dimensiones proveen a los refuerzos de una gran área superficial por unidad de volumen, lo que conduce a que con bajas cantidades de refuerzos se obtengan materiales con muy buenas propiedades.

En cuanto a los refuerzos, los hay de diferentes tamaños y formas, y pueden ser clasificados de acuerdo con la dimensionalidad de las partículas, en nanorefuerzos tipo nanofibras (1 dimensión), nanoláminas (2 dimensiones) y nanopartículas (3 dimensiones). Los nanorefuerzos tipo lámina son materiales bidimensionales, con un espesor de alrededor de $1 \mathrm{~nm}$, entre los que se encuentran las arcillas laminares y las láminas de grafeno. Las nanofibras poseen un diámetro menor a los $100 \mathrm{~nm}$, y entre ellas se pueden contar los nanotubos de carbono y la nanocelulosa. Finalmente, en las nanopartículas las 3 dimensiones se encuentran por debajo de los $100 \mathrm{~nm}$, siendo las más conocidas la sílice y los óxidos metálicos [97].

\subsubsection{Arcillas: montmorillonita y haloisita}

En este trabajo se utilizan, como refuerzos, dos nanoarcillas diferentes, montmorillonita y haloisita, las cuales se describen brevemente a continuación.

Por una parte, la montmorillonita es una arcilla formada por láminas, que consisten en una capa interior, formada por óxidos de aluminio y/o magnesio con coordinación octaédrica, que se ubica entre dos capas formadas por aniones silicato tetraédricos. Normalmente, el espacio entre las láminas, o galerías, se encuentra ocupado por iones de sodio, otros alcalinos y agua. Estos iones pueden ser intercambiados por cationes orgánicos de mayor tamaño, generalmente derivados de sales de amonio, para dar lugar a las arcillas orgánicamente modificadas. Estas modificaciones se introducen con la finalidad de incrementar la compatibilidad entre las arcillas, naturalmente hidrofílicas, y el polímero, por lo general hidrofóbico. Así mismo, la introducción de los modificadores en las arcillas produce un aumento del espaciado interlaminar, lo que puede facilitar la intercalación de las cadenas poliméricas, conduciendo a estructuras exfoliadas o intercaladas con buenas propiedades mecánicas y de barrera [87,99]. 
Por otra parte, la haloisita es un nanorefuerzo muy interesante ya que a su alta relación área/volumen aúna una estructura tubular, alta disponibilidad, biocompatibilidad y buena resistencia mecánica. Los nanotubos contienen algunos grupos silanol y aluminol en las caras externas e internas de los tubos, respectivamente, que permiten la modificación química de la arcilla. No obstante, la presencia de grupos $-\mathrm{OH}$ es relativamente baja, lo que otorga a la haloisita un carácter ligeramente hidrofóbico.

La haloisita natural posee algunas ventajas que podrían promover su utilización como refuerzos para nanocomposites poliméricos. En primer lugar, la ligera hidrofobicidad de esta arcilla podría facilitar su dispersión en las matrices poliméricas. Sin embargo, para obtener mejores grados de dispersión es necesaria la modificación de la haloisita. En segundo lugar, la arcilla posee interacciones partícula-partícula débiles, favoreciendo una dispersión uniforme de la arcilla, sin la necesidad de medios químicos para su separación. Por último, la distribución de los grupos - $\mathrm{OH}$ en las caras externa e interna de los nanotubos permite un amplio rango de modificaciones químicas, que podrían otorgar una mayor compatibilidad e incluso nuevas funcionalidades [68].

\subsubsection{Producción de los nanocomposites}

Existen diferentes alternativas para la fabricación de los nanocomposites basados en PLA. Entre dichos métodos se pueden encontrar:

- Procesado en fundido: este método es uno de los más estudiados, debido a su importancia a nivel industrial. En este proceso, se utilizan procedimientos convencionales, como el mezclado, en seco, del PLA y los refuerzos para su posterior procesado en fundido en equipos como extrusoras o mezcladores. Las condiciones de procesado deben ser cuidadosamente seleccionadas, ya que el PLA es susceptible de sufrir degradación termomecánica e hidrolítica, lo que perjudicaría las prestaciones del material final.

Este método es, desde un punto de vista de sostenibilidad medioambiental, muy ventajoso, ya que no utiliza solventes potencialmente perjudiciales para el medioambiente. Además, este método es relativamente fácil de llevar a escala industrial, en la que las extrusoras son usadas actualmente en la producción de materiales basados en PLA $[78,99]$.

- Métodos en disolución: este método es utilizado en las primeras etapas de investigación, a escala de laboratorio, y en el sector biomédico. Consiste en agregar una disolución del polímero a una dispersión de la arcilla, lo que genera fuertes interacciones entre ambas fases, y da a lugar a muy buenos grados de dispersión de la arcilla dentro de la matriz polimérica. Sin embargo, debido al alto consumo de solventes que implica, esta técnica se considera perjudicial desde un punto de vista medioambiental.

- Polimerización in situ: este proceso consiste en la polimerización del monómero en presencia de las arcillas, para dar lugar a una mayor compatibilidad y mejores prestaciones. En primer lugar, la arcilla es hinchada en el monómero en estado líquido, o en una disolución del monómero, que luego polimeriza dentro de las galerías, o los 
nanotubos, de las arcillas formando nanocomposites intercalados y exfoliados. La polimerización puede ser iniciada por catalizadores, calor o iniciadores.

- Otros métodos: los nanocomposites de PLA pueden ser también obtenidos mediante la mezcla de fibras de PLA y fibras naturales y/o sintéticas, co-extrusión y prensado en caliente $[78,99,107]$.

\subsubsection{Aplicaciones de los nanocomposites}

Como se ha mencionado anteriormente, la presencia de los refuerzos de escala nanométrica dispersos en la matriz de PLA ayuda a mejorar algunas propiedades del material, aumentando así su interés en algunas aplicaciones. Por ejemplo, la adición de pequeñas cantidades de arcilla produce una mejora en las propiedades de barrera del PLA, lo cual favorece su utilización en la fabricación de envases de alimentos. Así mismo, la adición de nanorefuerzos funcionales puede proveer de interesantes propiedades eléctricas, ópticas y electromagnéticas al PLA, haciéndolo un candidato a tener en cuenta en la fabricación de aparatos electrónicos, sensores, celdas solares, entre otros. Finalmente, si se emplean refuerzos biocompatibles, los nanocomposites de PLA podrían tener varias aplicaciones biomédicas como la ingeniería de tejidos o la fabricación de dispositivos para la liberación controlada de fármacos. En resumen, las aplicaciones de los nanocomposites del PLA son similares a las del PLA puro, aunque las mejores propiedades que exhiben los nanocomposites pueden promover la utilización de estos materiales en lugar de otros con un mayor impacto medioambiental, como pueden ser los polímeros basados en combustibles fósiles [8,99].

\subsection{Reciclado mecánico}

El reciclado mecánico consiste en la recuperación, empleando medios mecánicos, de desechos plásticos de post-consumo para su reutilización en procesos de manufactura de productos plásticos. Entre las etapas más frecuentes en este tipo de reciclado se encuentran el triturado, lavado, separación, secado y procesado en fundido (mediante extrusión o inyección) de los residuos plásticos [4].

El reciclado mecánico de los plásticos derivados del petróleo lleva en la palestra desde los años 70, pero es en los últimos años cuando se ha incrementado el interés debido a los crecientes problemas medioambientales y a las políticas de los organismos gubernamentales. Sin embargo, en el caso de los bioplásticos existen diferentes argumentos a favor y en contra del reciclado mecánico. Por una parte, entre los argumentos a favor de este proceso se pueden enumerar [81]:

- El desecho de los artículos hechos de bioplásticos implica el descarte de materias primas que podrían ser valiosas.

- El proceso de producción y transformación de las materias primas en bioplásticos conlleva un fuerte gasto energético, y tiene un impacto medioambiental considerable. 
- El reciclado mecánico puede jugar un papel crucial en la reducción del consumo de recursos renovables que actualmente son utilizados para la producción de los bioplásticos. - A pesar de que muchos bioplásticos, entre ellos el PLA, son considerados biodegradables, algunos de ellos no se degradan en condiciones normales, incluso con la presencia de microorganismos. Esto podría llevar a una importante acumulación de residuos, generando un problema medioambiental.

Por otra parte, los argumentos más destacables en contra del reciclado de bioplásticos son:

- La presencia de bioplásticos, como el PLA, puede contaminar otros plásticos presentes en las corrientes de reciclado, como el PET [62,71].

- Es necesaria una producción importante de bioplásticos, alrededor de 200000 toneladas/año para que el reciclado sea rentable económicamente [38].

Además de los problemas planteados anteriormente, uno de los retos más importantes a los que se enfrentan los recicladores es a la degradación de los plásticos durante el uso y el reciclado, debido a la exposición de los polímeros a agentes degradantes como el oxígeno, agua, radiación UV, esfuerzos mecánicos y temperatura. Esta degradación, en combinación con la heterogeneidad de los materiales obtenidos, produce un descenso de las prestaciones de los plásticos reciclados, relegándolos a aplicaciones poco exigentes, y dificultando la obtención de valor agregado a partir de estos materiales $[4,11,41]$.

\subsubsection{Etapas del reciclado mecánico}

El proceso de reciclado mecánico comprende varias etapas previas al reprocesado, entre las cuales se pueden contar: clasificación y separación, molienda o triturado y lavado y secado.

La separación y clasificación de los diferentes tipos de polímeros presentes en los residuos sólidos es una etapa crucial en el reciclado mecánico. Entre las técnicas utilizadas se pueden encontrar: separación manual, cuyo principal problema es su alto coste; separación por densidad; escaneo óptico, que es útil para la separación de los plásticos por su transparencia o color, pero que no provee información sobre la estructura química de los materiales; análisis de rayos X, que ha demostrado ser efectivo sólo para separar poli(cloruro de vinilo) (PVC); infrarrojo cercano (NIR), que permite diferenciar entre varios polímeros; y fluorescencia, que conlleva la utilización de marcadores para poder separar efectivamente los diferentes plásticos. Cabe destacar que incluso los plásticos separados pueden dar problemas en la etapa de reprocesado, debido a los diferentes grados de un mismo polímero presentes en el mercado.

El alto coste de la etapa de clasificación es uno de los factores limitantes a la hora de implantar procesos de reciclado mecánico para diferentes materiales, como los 
bioplásticos, ya que hasta hace relativamente poco tiempo no existía un volumen de material suficiente que justificara la inversión necesaria para este proceso [81].

La molienda o triturado es la etapa en la que los residuos son cortados y transformados en partículas más pequeñas, como hojuelas. Uno de los principales problemas que pueden surgir en esta etapa es la cantidad de calor generado, que puede fundir, o al menos ablandar, a algunos bioplásticos con bajas temperaturas de fusión y/o transición vítrea. Además, las temperaturas alcanzadas y los esfuerzos realizados podrían contribuir de forma adicional a la degradación de los polímeros más susceptibles a la degradación termomecánica $[4,81]$.

La etapa de lavado puede ser encontrada en muchos procesos de reciclado y se realiza con el objetivo de eliminar posibles impurezas y contaminantes presentes en el plástico de post-consumo. En algunos materiales, como el caso del PET empleado en el envasado de alimentos, se realiza un lavado enérgico en presencia de hidróxido de sodio y surfactantes si se pretende volver a utilizar el plástico en envasado de alimentos [32].

Tras el lavado es necesaria una etapa de secado, la cual es crucial en el caso de algunos bioplásticos como el PLA, ya que la presencia de agua durante el reprocesado conduce a la degradación hidrolítica del polímero, afectando negativamente las prestaciones del material reciclado. Esta etapa puede ser problemática en el caso de materiales con bajos valores de la temperatura de transición vítrea, $T_{g}$, y que no han sido cristalizados, ya que los tiempos de secado pueden ser muy largos [81]. 


\section{METODOLOGÍA}

\subsection{Materiales}

En esta tesis se ha utilizado un grado comercial de PLA especialmente diseñado para aplicaciones de envasado de alimentos, concretamente, el Ingeo ${ }^{\mathrm{TM}}$ Biopolymer 2003D, fabricado por NatureWorks. Este polímero posee un índice de fluidez de $6 \mathrm{~g} / 10$ min (medidos a $210{ }^{\circ} \mathrm{C}$ y con 2,16 kg de presión), y una proporción isómero d del $4 \%$.

Para la fabricación de los nanocomposites, se han utilizado diferentes arcillas. En primer lugar, una montmorillonita orgánicamente modificada, disponible comercialmente bajo el nombre de Cloisite C30B, suministrada por Southern Clay Products. El modificador orgánico de esta arcilla se muestra en la Fig. 3, y consiste en un cloruro de amonio cuaternario, cuyos sustituyentes son dos grupos hidroxietilo, un grupo metilo y un radical de gran tamaño. Se ha utilizado también una haloisita sin modificar, que ha sido suministrada por Aldrich.

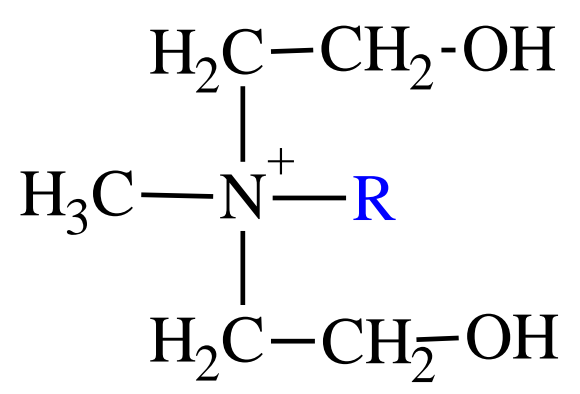

Fig. 3. Modificación orgánica de la arcilla Cloisite C30B.

Además del PLA y las arcillas se han utilizado otros reactivos, tales como:

- Peróxido de dicumilo (DCP), suministrado por Alfa Aesar.

- 3-aminopropiltrietoxisilano (APS), suministrado por Argos Organics.

- Extensor de cadena (no disponible comercialmente), suministrado por Clariant.

- Cloroformo, suministrado por Merck.

- Fosfato de sodio dibásico y fosfato de sodio monobásico, suministrados por SigmaAldrich. 


\subsection{Obtención de materiales vírgenes y reciclados}

El proceso de obtención de los diferentes materiales, tanto vírgenes como reciclados, se encuentra resumido en el esquema presentado en la Fig. 4. Es importante destacar que antes de cada proceso de extrusión, el PLA fue cristalizado en una estufa de vacío a $100{ }^{\circ} \mathrm{C}$, durante 20 minutos. Posteriormente, tanto las arcillas como el PLA cristalizado fueron secados durante dos horas en una estufa de vacío a $85^{\circ} \mathrm{C}$, con el objetivo de eliminar la humedad del material, y reducir la degradación hidrolítica durante el procesado.

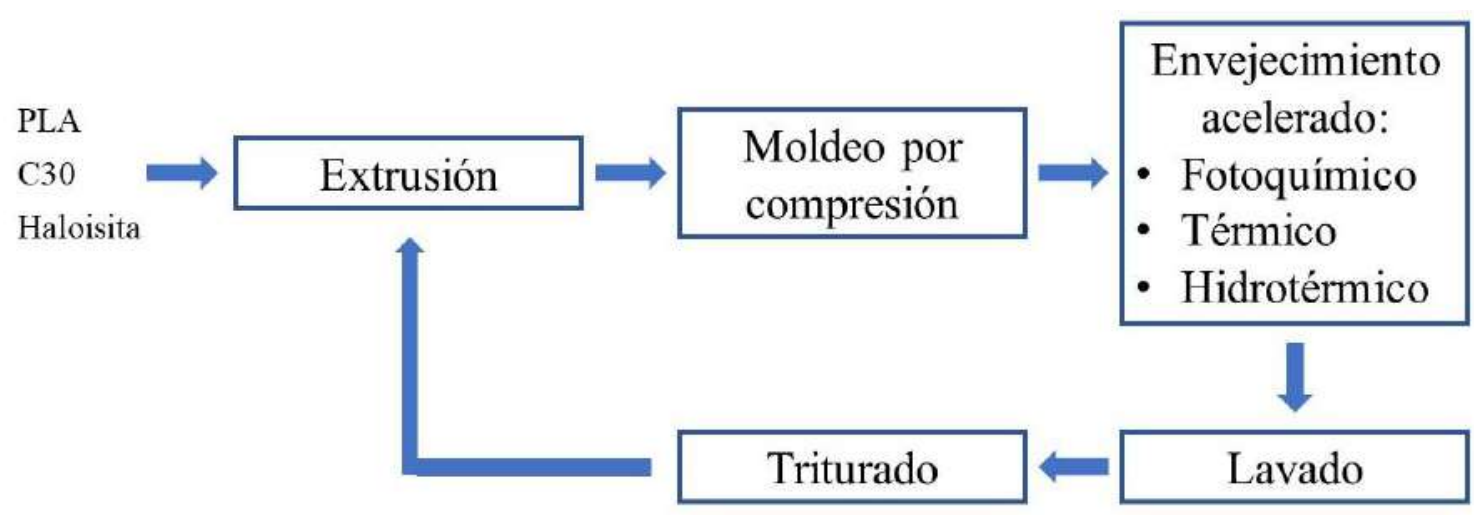

Fig. 4. Esquema de la simulación de los diferentes procesos de reciclado.

Como se puede observar en la Fig. 4, el PLA y los nanocomposites con $2 \%$ de C 30 y $2 \%$ de haloisita fueron, en primer lugar, procesados mediante extrusión. Dicho proceso fue llevado a cabo en una microextrusora de doble husillo Rondol Microlab Twin Screw, con una relación $\mathrm{L} / \mathrm{D}=20$, y con una velocidad de $60 \mathrm{rpm}$. El perfil de temperaturas, desde la alimentación hasta la boquilla, fue 125-160-190-190-180 ${ }^{\circ} \mathrm{C}$. Los materiales obtenidos fueron luego transformados en filmes de $230 \pm 20 \mu \mathrm{m}$ de espesor utilizando una prensa de platos calientes IQAP-LAP, a $190^{\circ} \mathrm{C}$, de acuerdo con el siguiente protocolo: una etapa de fusión, sin presión, durante 5 minutos; una etapa de desgasificación de 2 minutos de duración; y, finalmente, una etapa de enfriamiento en platos refrigerados, con una presión de $14 \mathrm{MPa}$, durante 5 minutos.

Una vez obtenidos los filmes, una parte de ellos fue sometida a un proceso de envejecimiento acelerado, cuya finalidad era simular la degradación sufrida por los materiales durante su vida útil. Este proceso de envejecimiento se dividió en tres etapas:

- Envejecimiento fotoquímico: llevada a cabo en una cámara Atlas UVCON, equipada con 8 lámparas F40UVB. El tiempo de exposición fue de 40 horas.

- Envejecimiento térmico: llevada a cabo en una estufa a $50{ }^{\circ} \mathrm{C}$. El tiempo de exposición fue de 468 horas. 
- Envejecimiento hidrotérmico: los materiales fueron sumergidos en agua destilada, a $30^{\circ} \mathrm{C}$, durante 10 días.

Una vez envejecidas, las muestras fueron lavadas en una solución de hidróxido de sodio ( $1 \%$ en peso) y un surfactante (Tritón $\mathrm{X}, 0,3 \%$ en peso), a $85^{\circ} \mathrm{C}$, durante 15 minutos. Cabe destacar que estas condiciones son similares a las aplicadas en plásticos dedicados a la fabricación de envases de alimentos [32]. Después del proceso de lavado, los materiales fueron secados y triturados usando un molino criogénico, para posteriormente someterlos a un segundo reprocesado mediante extrusión y moldeo por compresión. La Tabla 1 recoge los materiales obtenidos, según las etapas del proceso de reciclado simulado a las que fueron sometidos.

Tabla 1. Materiales obtenidos en los diferentes procesos de reciclado simulado

\begin{tabular}{|c|c|}
\hline Material & Descripción \\
\hline PLAV & $\begin{array}{l}\text { PLA sometido a una etapa de procesado por extrusión y } \\
\text { moldeo por compresión }\end{array}$ \\
\hline PLAR & $\begin{array}{c}\text { PLAV sometido a las etapas de envejecimiento fotoquímico y } \\
\text { térmico, y reprocesado }\end{array}$ \\
\hline PLARL & $\begin{array}{c}\text { PLAV sometido a las etapas de envejecimiento fotoquímico y } \\
\text { térmico, lavado y reprocesado }\end{array}$ \\
\hline PLARLH & $\begin{array}{l}\text { PLAV sometido a las etapas de envejecimiento fotoquímico, } \\
\text { térmico e hidrotérmico, lavado y reprocesado }\end{array}$ \\
\hline PLAV-C30 & $\begin{array}{c}\text { Nanocomposite de PLA con } 2 \% \text { de C } 30 \text { sometido a una etapa } \\
\text { de procesado por extrusión y moldeo por compresión }\end{array}$ \\
\hline PLAR-C30 & $\begin{array}{c}\text { Nanocomposite de PLA con } 2 \% \text { de C30 sometido a las etapas } \\
\text { de envejecimiento fotoquímico y térmico, y reprocesado }\end{array}$ \\
\hline PLARL-C30 & $\begin{array}{c}\text { Nanocomposite de PLA con } 2 \% \text { de C30 sometido a las etapas } \\
\text { de envejecimiento fotoquímico y térmico, lavado y } \\
\text { reprocesado }\end{array}$ \\
\hline PLAV-Ha & $\begin{array}{l}\text { Nanocomposite de PLA con } 2 \% \text { de haloisita sometido a una } \\
\text { etapa de procesado por extrusión y moldeo por compresión }\end{array}$ \\
\hline PLAR-Ha & $\begin{array}{c}\text { Nanocomposite de PLA con } 2 \% \text { de haloisita sometido a las } \\
\text { etapas de envejecimiento fotoquímico y térmico, y } \\
\text { reprocesado }\end{array}$ \\
\hline
\end{tabular}

\subsection{Mejora de las propiedades del PLA reciclado}

La Fig. 5 muestra el proceso seguido para la recuperación de las propiedades del PLA reciclado mecánicamente. Se puede observar que el PLA, sometido a un primer procesado, a un envejecimiento acelerado y a una etapa de lavado, es reprocesado junto con diferentes aditivos y/o refuerzos. 


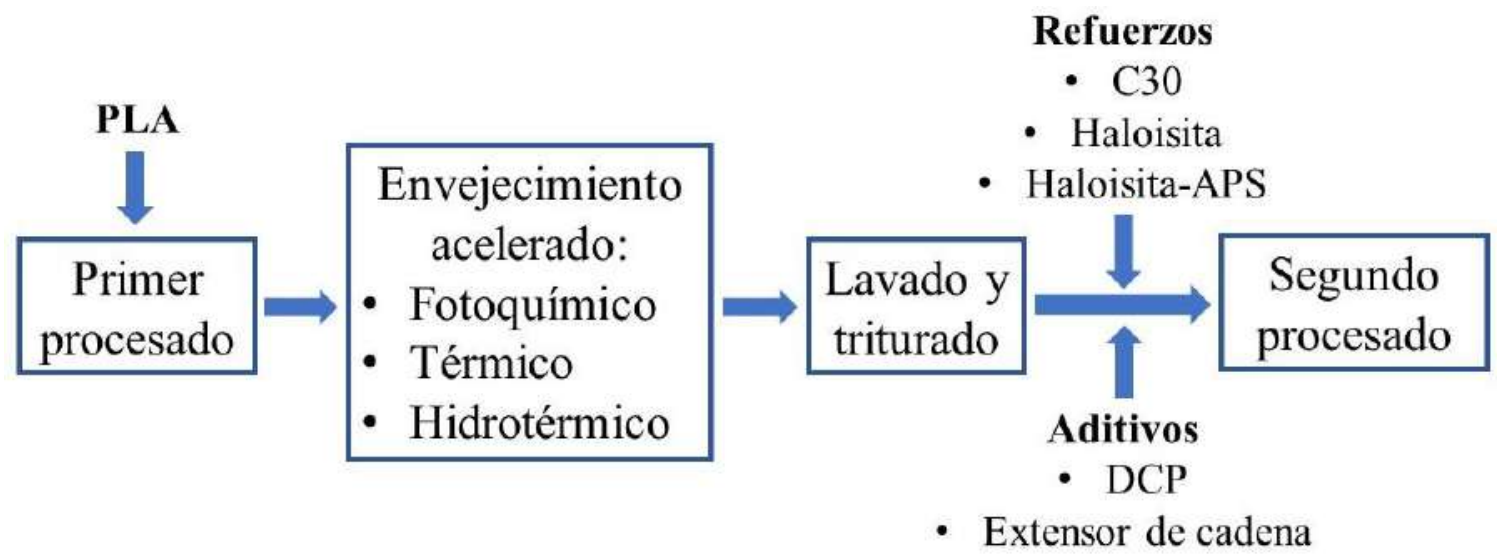

Fig. 5. Esquema del proceso de mejora de propiedades del PLA reciclado.

Las alternativas estudiadas en este trabajo para la recuperación de las propiedades del PLA pueden dividirse en dos grupos: arcillas y aditivos. En el primer grupo se encuentran Cloisite C30B, haloisita sin modificar y haloisita modificada con APS (el proceso de modificación se describe en la sección 3.4). En el segundo grupo se encuentran compuestos que bien permiten aumentar el peso molecular del PLA, o bien promueven reacciones de entrecruzamiento entre las cadenas del PLA reciclado, tales como: peróxido de dicumilo, trifenilfosfito y un extensor de cadena comercial, CESA®-extend BLA0025041-ZN (suministrado por Clariant).

Tabla 2. Materiales obtenidos según los diferentes métodos para la recuperación de propiedades del PLA reciclado

\begin{tabular}{|c|c|}
\hline Material & Descripción \\
\hline PLARLH & $\begin{array}{l}\text { PLAV sometido a las etapas de envejecimiento fotoquímico, } \\
\text { térmico e hidrotérmico, lavado y reprocesado }\end{array}$ \\
\hline PLARLH-VC & $\begin{array}{c}\text { PLAV sometido a las etapas de envejecimiento fotoquímico y } \\
\text { térmico, y luego reprocesado con } 50 \% \text { de PLA virgen y } 1,5 \% \\
\text { de un extensor de cadena comercial }\end{array}$ \\
\hline PLARLH-DCP & $\begin{array}{l}\text { PLAV sometido a las etapas de envejecimiento fotoquímico y } \\
\text { térmico, y luego reprocesado con } 0.3 \% \text { de peróxido de } \\
\text { dicumilo }\end{array}$ \\
\hline PLARLH-C30 & $\begin{array}{l}\text { PLAV sometido a las etapas de envejecimiento fotoquímico y } \\
\text { térmico, y luego reprocesado con } 2 \% \text { de C30 }\end{array}$ \\
\hline PLARLH-Ha & $\begin{array}{l}\text { PLAV sometido a las etapas de envejecimiento fotoquímico y } \\
\text { térmico, y luego reprocesado con } 2 \% \text { de haloisita }\end{array}$ \\
\hline PLARLH-HaM & $\begin{array}{l}\text { PLAV sometido a las etapas de envejecimiento fotoquímico y } \\
\text { térmico, y luego reprocesado con } 2 \% \text { de haloisita modificada }\end{array}$ \\
\hline
\end{tabular}




\subsection{Modificación de la haloisita con 3-aminopropiltrietoxisilano}

La modificación de los nanotubos de haloisita se ha realizado de acuerdo con el procedimiento descrito en la literatura [61]. Se tomaron $2 \mathrm{~g}$ de haloisita, previamente secada a $80^{\circ} \mathrm{C}$ durante 12 horas, y se añadieron a $200 \mathrm{~mL}$ de una mezcla etanol/agua (75/25) ( $\mathrm{vol} / \mathrm{vol})$. La disolución resultante fue sometida a ultrasonidos con punta sónica durante 1 hora. A continuación, se añadieron $4 \mathrm{~g}$ de APS y se ajustó a $\mathrm{pH}=5$ añadiendo ácido acético. La mezcla se mantuvo sometida a ultrasonidos con la punta sónica durante 3 horas. La separación de la arcilla modificada se realizó mediante sucesivos centrifugados a $6800 \mathrm{rpm}$ y lavados con agua, para eliminar el exceso de APS. Finalmente, la arcilla modificada fue secada a $80^{\circ} \mathrm{C}$ durante 24 horas.

\subsection{Ensayos de absorción de agua y degradación hidrolítica}

Para la realización de los ensayos de absorción de agua y resistencia frente a la degradación hidrolítica, se utilizaron muestras de 4 x 2,5 cm, con un espesor (230 \pm 20$)$ $\mu \mathrm{m}$ de diferentes materiales vírgenes y reciclados. Dichas muestras fueron secadas en una estufa de vacío, a $40^{\circ} \mathrm{C}$, durante dos días, para eliminar el exceso de humedad. Luego, las muestras secas fueron sumergidas en recipientes con $100 \mathrm{ml}$ de una disolución reguladora de fosfatos $(0,05 \mathrm{M} \mathrm{y} \mathrm{pH} \mathrm{7,4 \pm 0,2),} \mathrm{que} \mathrm{fueron} \mathrm{colocados} \mathrm{en} \mathrm{estufas} \mathrm{a} 37$ ó $58{ }^{\circ} \mathrm{C}$. A diferentes tiempos de inmersión, las muestras fueron extraídas para su caracterización.

Para determinar la cantidad de agua absorbida a un tiempo $t, M_{t}$, se empleó la siguiente ecuación:

$$
M_{t}(\%)=\frac{W_{t}-W_{0}}{W_{0}} \times 100
$$

donde $W_{t}$ es la masa de la muestra a un tiempo $t$ y $W_{0}$ es la masa inicial de la muestra, antes de ser sumergida en la disolución reguladora.

Una vez determinada la cantidad de agua absorbida por las muestras, estas fueron secadas en una estufa de vacío a $40{ }^{\circ} \mathrm{C}$ durante 7 días, y caracterizadas según las técnicas mencionadas en la sección 3.6.

\subsection{Técnicas de caracterización}

Se ha medido la viscosidad intrínseca de las diferentes muestras utilizando un viscosímetro Ubbelohde y cloroformo como solvente, a una temperatura de $25^{\circ} \mathrm{C}$.

Se ha realizado espectroscopía infrarroja de las diferentes muestras utilizando un espectrofotómetro Nicolet iS10, equipado con un accesorio de reflectancia total atenuada (ATR). Se utilizaron 16 barridos y una resolución de $4 \mathrm{~cm}^{-1}$ para la caracterización de los 
materiales, y 25 barridos con una resolución de $2 \mathrm{~cm}^{-1}$ para el estudio de las formas cristalinas. Los espectros fueron corregidos usando el software OMNIC 9.2.41, y han sido normalizados utilizando la banda de absorción ubicada a $1450 \mathrm{~cm}^{-1}$, la cual es empleada usualmente como una referencia interna en el PLA [59]. A partir de los espectros infrarrojos de las diferentes muestras se estimó el grado de cristalinidad, $X_{C}$, empleando la siguiente ecuación [72]:

$$
X_{C}=\frac{I_{0}-I_{f}}{I_{0}} \times 100
$$

donde $I_{0}$ e $I_{f}$ son el área de la banda de absorción a $955 \mathrm{~cm}^{-1}$ antes y después de la cristalización respectivamente.

En el caso de los nanocomposites, la morfología de los materiales fue estudiada mediante microscopía electrónica de transmisión, utilizando un microscopio JEOL JEM2100 operado a $200 \mathrm{kV}$. También se ha utilizado microscopía electrónica de barrido, en un microscopio Hitachi SU8000; cabe destacar que las muestras fueron previamente recubiertas con una película de oro/paladio.

Para los ensayos de difracción de rayos $\mathrm{X}$ de las diferentes muestras se ha empleado un difractómetro Bruker D8, equipado con un generador $\mathrm{CuK} \alpha(\lambda=0,1542 \mathrm{~nm})$ a $40 \mathrm{kV}$ y $4 \mathrm{~mA}$. También se ha empleado un difractómetro X'PERT-MPD equipado con un generador $\mathrm{CuK} \alpha\left(\lambda_{1}=0,154056 \mathrm{~nm}\right.$ y $\left.\lambda_{2}=0,154439 \mathrm{~nm}\right)$ a $45 \mathrm{kV} 740 \mathrm{~mA}$. El espaciado basal se determina utilizando la Ley de Bragg, que se presenta la siguiente ecuación [22]:

$$
\lambda=2 d \sin \theta
$$

donde $\lambda$ es la longitud de onda de los rayos, $d$ es el espaciado basal y $\theta$ es el ángulo entre los rayos y la muestra.

El grado de cristalinidad puede ser estimado, a partir de los difractogramas de rayos $\mathrm{X}$, utilizando la ecuación 4 :

$$
X_{C}=\frac{I_{c}}{I_{c}+I_{a}}
$$

donde $I_{c}$ e $I_{a}$ son las áreas de las difracciones de las fases cristalina y amorfa respectivamente.

A partir de los ensayos XRD también se puede calcular el contenido relativo de la forma cristalina $\alpha, C_{\alpha}, \mathrm{y} \alpha^{\prime}$, usando la siguiente ecuación [85]: 
$C_{\alpha}=\left[\frac{d_{\alpha \prime}-d_{\text {muestra }}}{d_{\alpha \prime}-d_{\alpha}}\right] \times 100$

donde $d$ es el espaciado reticular, determinado a partir de los difractogramas de rayos X, de las formas $\alpha^{\prime}, \alpha, y$ de la muestra, respectivamente, a diferentes tiempos de inmersión.

Los ensayos de calorimetría diferencial de barrido de los materiales sin sumergir fueron realizados usando un calorímetro TA Q20. Se emplearon muestras de aproximadamente $5 \mathrm{mg}$, capsulas de aluminio y atmósfera de $\mathrm{N}_{2}$. El protocolo seguido para el ensayo fue el siguiente:

- $\quad$ Primer barrido de calentamiento, a $5^{\circ} \mathrm{C} / \mathrm{min}$, desde 30 hasta $180^{\circ} \mathrm{C}$.

- Un paso isotérmico, a $180^{\circ} \mathrm{C}$, durante 3 minutos para borrar la historia térmica.

- Un barrido de enfriamiento, a $5^{\circ} \mathrm{C} / \mathrm{min}$, desde 180 hasta $0{ }^{\circ} \mathrm{C}$.

- Un segundo barrido de calentamiento, a $5^{\circ} \mathrm{C} / \mathrm{min}$, desde 0 hasta $180{ }^{\circ} \mathrm{C}$.

La monitorización de los cambios estructurales de los materiales durante la inmersión fue realizada en un calorímetro Mettler-Toledo 823e, siguiendo el protocolo anteriormente explicado, pero con una velocidad de calentamiento y enfriamiento de 10 ${ }^{\circ} \mathrm{C} / \mathrm{min}$.

El grado de cristalinidad, $X_{C}$, de las diferentes muestras fue calculado empleando la siguiente ecuación:

$$
X_{C}(\%)=\frac{\Delta H_{f}-\Delta H_{c}-\Delta H_{\alpha \prime \rightarrow \alpha}}{\Delta H_{\infty}} \times 100
$$

donde $\Delta \mathrm{H}_{\mathrm{f}}, \Delta \mathrm{H}_{\mathrm{c}}$ y $\Delta \mathrm{H}_{\alpha^{\prime} \rightarrow \alpha}$ son las entalpías de fusión, cristalización y transición $\alpha^{\prime}-\alpha$, respectivamente. $\Delta \mathrm{H}_{\infty}$ es la entalpía de fusión de un PLLA $100 \%$ cristalino, que tiene un valor de 93,1 J/g [66].

El análisis termogravimétrico de los diferentes materiales fue realizado en una termobalanza TA Instruments TGA2050, utilizando muestras de aproximadamente 14 $\mathrm{mg}$, las cuales fueron calentadas desde $40{ }^{\circ} \mathrm{C}$ hasta $800{ }^{\circ} \mathrm{C}$, a una velocidad de $10{ }^{\circ} \mathrm{C} / \mathrm{min}$ y bajo atmósfera de $\mathrm{N}_{2}(30 \mathrm{~mL} / \mathrm{min})$.

El espectro de absorción UV de los medios de inmersión fue medido en un espectrofotómetro UV-Vis Perkin-Elmer Lambda 35, con una velocidad de barrido de $480 \mathrm{~nm} / \mathrm{min}$. La transmisión de luz en la región visible de las diferentes muestras fue medida de acuerdo a la norma ISO 13468 [55], usando un espectrofotómetro UV-Vis Shimadzu 2401 PC, equipado con una esfera integradora Shimadzu, y utilizando una velocidad de barrido de $200 \mathrm{~nm} / \mathrm{min}$. Cabe destacar que todos los espectros fueron normalizados para considerar el espesor de cada muestra.

Las medidas de microdureza de las muestras fueron realizadas a temperatura ambiente en un microdurómetro Shimadzu Type M. Se empleó un indentador piramidal tipo Vickers, aplicando una carga de $25 \mathrm{~g}$ durante $10 \mathrm{~s}$. 
Las propiedades de barrera frente a gases de los diferentes materiales fueron estudiadas utilizando una celda de permeabilidad fabricada en el laboratorio [6]. El instrumento consiste en una celda con dos cámaras, separadas por una membrana (de espesor conocido) del material estudiado. La permeabilidad del $\mathrm{N}_{2}, \mathrm{O}_{2}$ y $\mathrm{CO}_{2}$ fue determinada a $30{ }^{\circ} \mathrm{C}$ mediante experimentos de difusión a través de una membrana previamente desgasificada. Después de la desgasificación, una variación de 0,2 MPa en la presión fue impuesta en el lado de alta presión de la membrana, mientras se medía la presión en el lado de baja presión. El coeficiente de permeabilidad, $P$, fue estimado a partir de la pendiente de la recta presión vs. tiempo una vez alcanzado el estado estacionario [102]. 


\section{EFECTO DEL RECICLADO MECÁNICO EN LA ESTRUCTURA Y PROPIEDADES DEL PLA Y SUS NANOCOMPOSITES}

El PLA es susceptible de sufrir degradación termomecánica, fotoquímica e hidrolítica durante su procesado, vida útil y reciclado mecánico, lo que podría afectar la estructura y las propiedades del plástico reciclado, comprometiendo por tanto su reciclabilidad. Uno de los objetivos de las tesis es evaluar el efecto de la degradación en uso y de diferentes procesos de reciclado en la estructura y algunas propiedades relevantes en el campo del envasado de alimentos del PLA. A continuación, se presentan y analizan los resultados obtenidos tanto para el PLA como para sus nanocomposites con C30 y haloisita.

\subsection{Viscosidad intrínseca de los materiales vírgenes y reciclados}

Uno de los parámetros más importantes desde el punto de vista del procesado en fundido de polímeros es la viscosidad intrínseca. Esto se debe a que, a nivel industrial, los equipos de procesado (extrusoras, inyectoras, etc.) están diseñados para trabajar en condiciones específicas, por lo que una variación significativa de la viscosidad durante el reciclado podría afectar el proceso de producción y la calidad de los materiales obtenidos. Así mismo, la viscosidad intrínseca está relacionada con el peso molecular del polímero, factor que juega un papel muy importante en las propiedades térmicas, mecánicas y de barrera del material. Por estas razones es muy importante analizar el efecto de los diferentes procesos de reciclado mecánico en la viscosidad intrínseca del PLA y sus nanocomposites.

En la Fig. 6 se presentan los valores de viscosidad intrínseca de la granza de PLA sin procesar y del PLA sometido a diferentes procesos de reciclado mecánico. Se puede observar que el PLAV tiene una viscosidad ligeramente inferior (alrededor del 6\%) que la granza sin procesar. Este comportamiento se ajusta a lo esperado en el PLA, ya que es 
un material susceptible a la degradación termomecánica durante el procesado, debido a los esfuerzos de cizalla y a las altas temperaturas a las que es sometido el polímero. Este proceso puede, en general, seguir diferentes mecanismos como hidrólisis, transesterificación inter e intramolecular y ruptura aleatoria de la cadena principal por oxidación. Estas reacciones conducen a la generación de cadenas más cortas, disminuyendo el peso molecular y la viscosidad intrínseca del material.

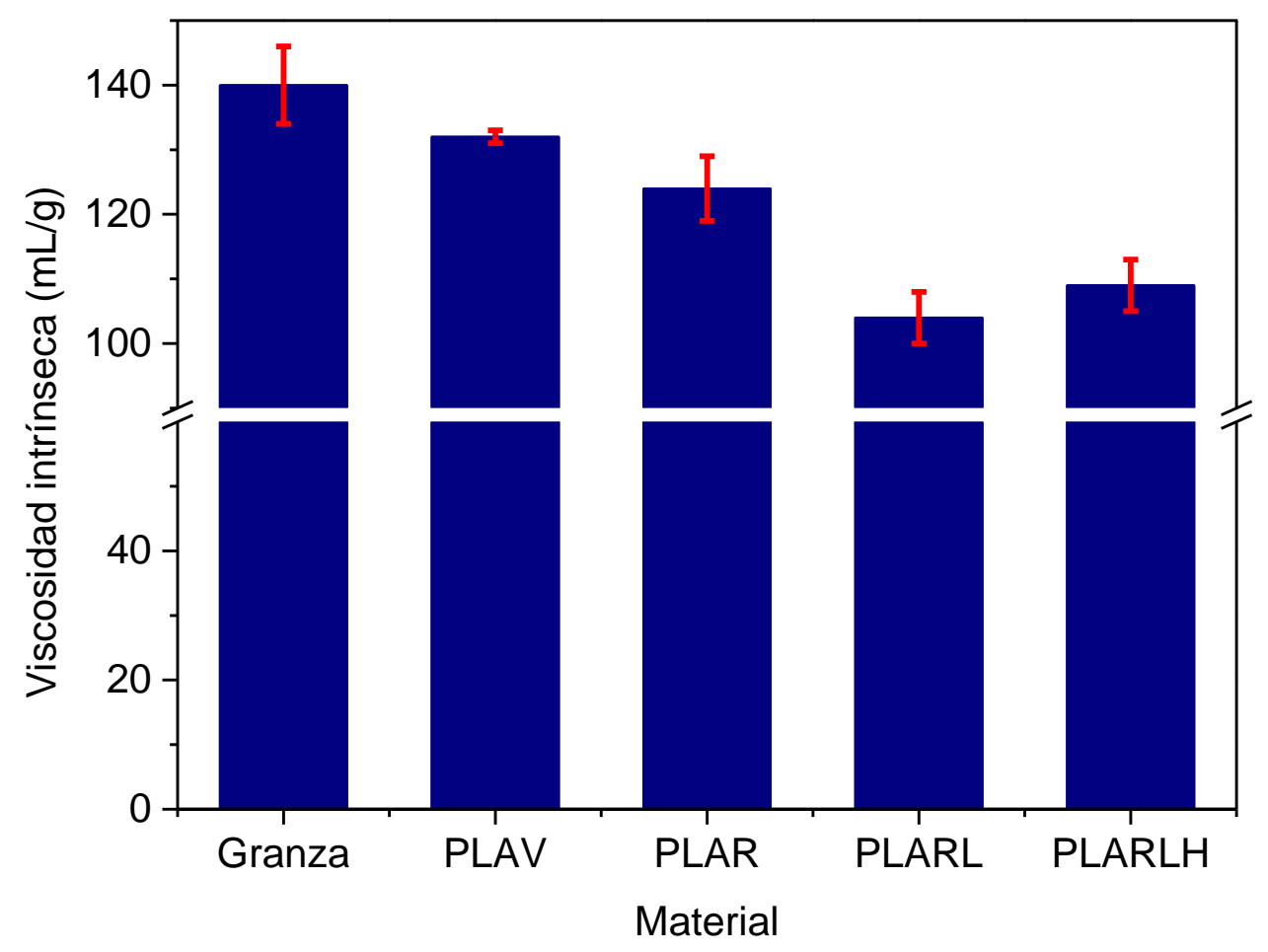

Fig. 6. Valores de viscosidad intrínseca del PLA sometido a diferentes procesos de reciclado mecánico.

En cuanto al efecto de los diferentes procesos de reciclado en la viscosidad intrínseca del PLA, en primer lugar, se puede observar que el PLAR presenta una viscosidad 6\% menor que el PLAV. Este descenso en la viscosidad intrínseca es comparable a los observados en la literatura para el PLA sometido a un ciclo de reprocesado mecánico (sin etapas de envejecimiento acelerado ni lavado). Por ejemplo, Badía et al. [14] reportan un descenso del 8,5\% en la viscosidad intrínseca de un PLA sometido a dos ciclos consecutivos de inyección. El descenso en la viscosidad intrínseca observado en el PLAR es consecuencia de la degradación del polímero durante el envejecimiento y durante el reprocesado. En cualquier caso, la variación es pequeña, por lo que el efecto del reciclado mecánico en las propiedades también debería ser limitado.

Sin embargo, si se observan los valores de viscosidad del PLARL y del PLARLH, se puede encontrar que el descenso de la viscosidad intrínseca es significativamente mayor (alrededor del $20 \%$ en ambos casos) que el observado en la literatura, en trabajos 
donde solo se reprocesa el material, indicando que la etapa de lavado juega un papel muy importante en la degradación del PLA durante el reciclado mecánico. Con el objetivo de analizar con mayor profundidad el efecto de la etapa de lavado en el reciclado, se han realizado medidas de viscosidad del PLA envejecido térmica y fotoquímicamente y lavado, pero sin reprocesar. El valor obtenido, $(123 \pm 1) \mathrm{mL} / \mathrm{g}$, se encuentra próximo al del PLAV, $(132 \pm 1) \mathrm{mL} / \mathrm{g}$, indicando que, si bien el polímero ha sufrido cierta degradación durante el envejecimiento y el lavado, la mayor parte de la pérdida en el peso molecular tiene lugar durante el reprocesado por extrusión. Si se considera que todas las muestras han sido secadas adecuadamente antes de cada etapa de extrusión, los resultados sugieren que la etapa de lavado debilita la estructura del PLA, haciéndolo más susceptible a sufrir degradación termomecánica durante el reprocesado y, por lo tanto, ocasionando un mayor descenso en la viscosidad y el peso molecular $[18,19]$.

El efecto de los diferentes procesos de reciclado en la viscosidad del PLA también ha sido medido en diferentes nanocomposites. En la Fig. 7 se presentan los valores de viscosidad intrínseca de los nanocomposites de PLA con C30 y haloisita. Se puede observar que en el PLAV-C30 y el PLAV-Ha el descenso de la viscosidad con respecto a la granza de PLA sin procesar es mayor que en el caso del PLA sin reforzar (11\% para el PLAV-C30 y 9\% para el PLAV-Ha, comparado con el 6\% del PLAV). Esta mayor degradación en nanocomposites basados en PLA ya ha sido reportada previamente en la literatura. Carrasco et al. [26] reportaron descensos en el peso molecular de nanocomposites de PLA cargados con 0,5 y 2,5\% de C30. Dicho descenso fue atribuido al potencial efecto de catálisis de las arcillas en la degradación del PLA, bien por hidrólisis (debido a la hidrofilicidad de las arcillas) o por aminólisis (debido a la presencia de modificadores orgánicos). Un resultado similar fue obtenido por Scaffaro et al. [103] en sistemas PLA-hidrotalcita, atribuyendo el mayor descenso a procesos de hidrólisis, degradación radicalaria o transesterificación. 


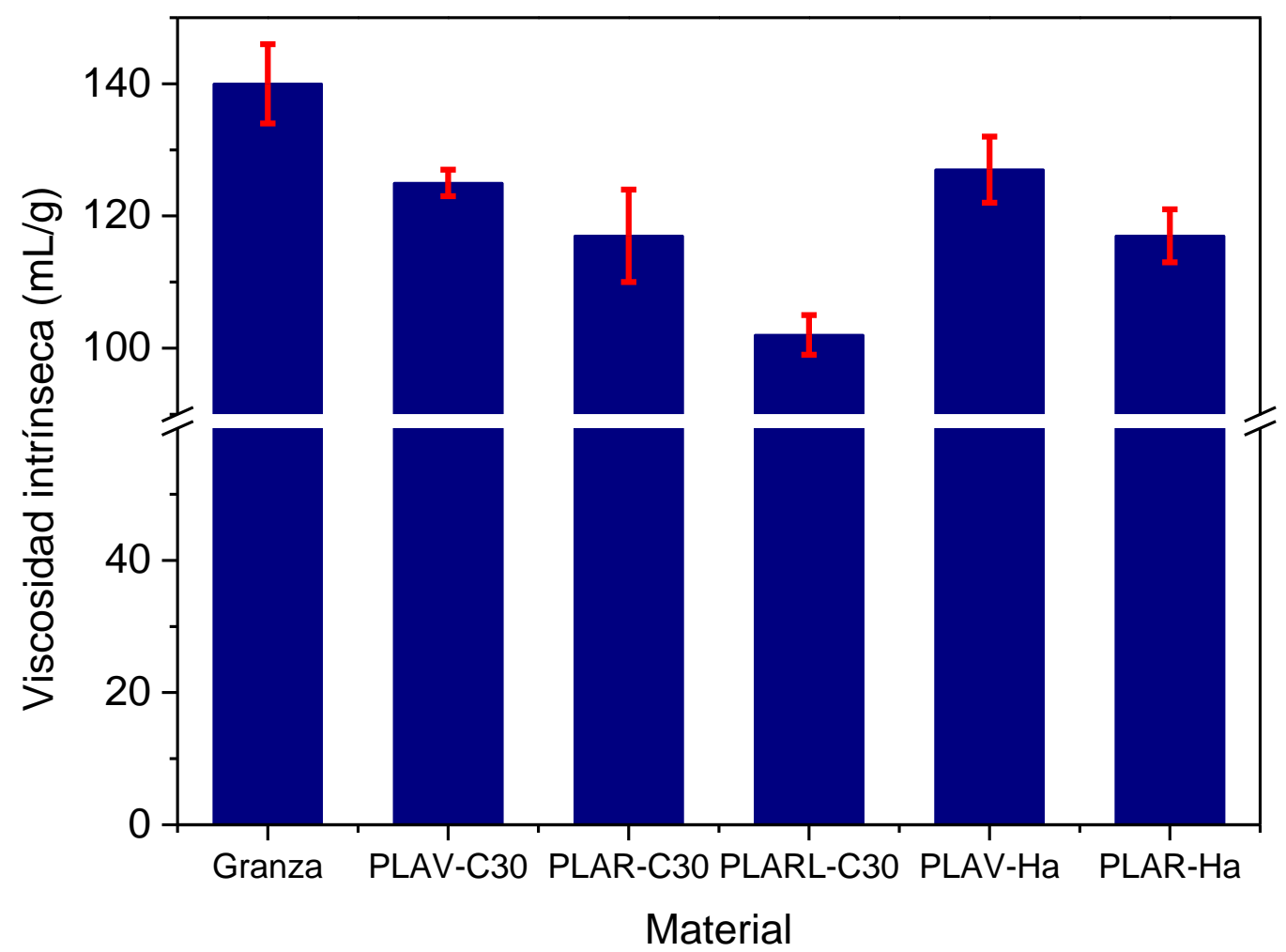

Fig. 7. Viscosidad intrínseca de los diferentes nanocomposites basados en PLA.

En cuanto al efecto del reciclado en los nanocomposites, se puede apreciar en la Fig. 7 que el comportamiento de estos materiales es similar al del PLA puro, presentando la mayor degradación el nanocomposite sometido a la etapa de lavado enérgico. Sin embargo, los valores de viscosidad de los nanocomposites reciclados son ligeramente inferiores los del PLA puro sometido a los mismos procesos de reciclado. Este comportamiento podría estar relacionado con el ya mencionado efecto catalítico de las arcillas en la degradación del PLA. Cabe también destacar que, aunque los valores de viscosidad de los materiales con C30 y haloisita son muy parecidos, parece haber una mayor degradación en el material con la montmorillonita. Dicho comportamiento podría estar relacionado con la presencia de un modificador orgánico en la montmorillonita (que no se encuentra en la haloisita), que mejora la dispersión de la arcilla en la matriz polimérica, y que incluso podría catalizar reacciones de degradación del PLA.

En resumen, los resultados de viscosidad intrínseca indican que el reciclado mecánico causa degradación del PLA, en especial cuando el material es sometido a una etapa de lavado intenso, por lo tanto, este tipo de procesos debe ser incluido en el estudio del reciclado mecánico, sobre todo para materiales con aplicaciones en el campo del envasado de alimentos. No obstante, aunque el reciclado ocasiona degradación del PLA, los descensos de viscosidad son relativamente pequeños, sugiriendo que tanto el PLA como sus nanocomposites podrían soportar un ciclo de reciclado mecánico, y que este no debería tener un impacto demasiado alto en las prestaciones del material. 


\subsection{Morfología de los nanocomposites vírgenes y reciclados}

Se ha estudiado la morfología de los nanocomposites, tanto vírgenes como reciclados, con el objetivo de determinar el efecto del reciclado mecánico en la dispersión de las arcillas en el material, ya que esta juega un papel muy importante en las propiedades ópticas, térmicas, mecánicas y de barrera de los materiales reciclados. Este análisis se ha realizado utilizando técnicas de microscopía electrónica (TEM y SEM) y de difracción de rayos $\mathrm{X}(\mathrm{XRD})$.

En la Fig. 8 se muestran las fotografías TEM de los nanocomposites de PLA con C30 virgen y reciclado (sin etapa de lavado). Se puede observar que ambos materiales presentan algunos agregados de arcilla, pero el tamaño de partícula parece ser menor en el nanocomposite reciclado que en el virgen. Esta mejora en la dispersión podría estar relacionada con el mayor tiempo de residencia del material en la extrusora (como consecuencia de la etapa de reprocesado), durante los que se aplican altos esfuerzos de cizalla que favorece el mezclado de la fase dispersa. Además, la degradación del polímero genera cadenas más cortas, las cuales pueden penetrar mejor entre las láminas de arcilla, mejorando también la dispersión. Resultados similares han sido reportados por Scaffaro et al. [104], tras someter mezclas de PLA con 5\% de hidrotalcita modificada orgánicamente a dos ciclos de extrusión consecutivos en una extrusora de doble husillo.

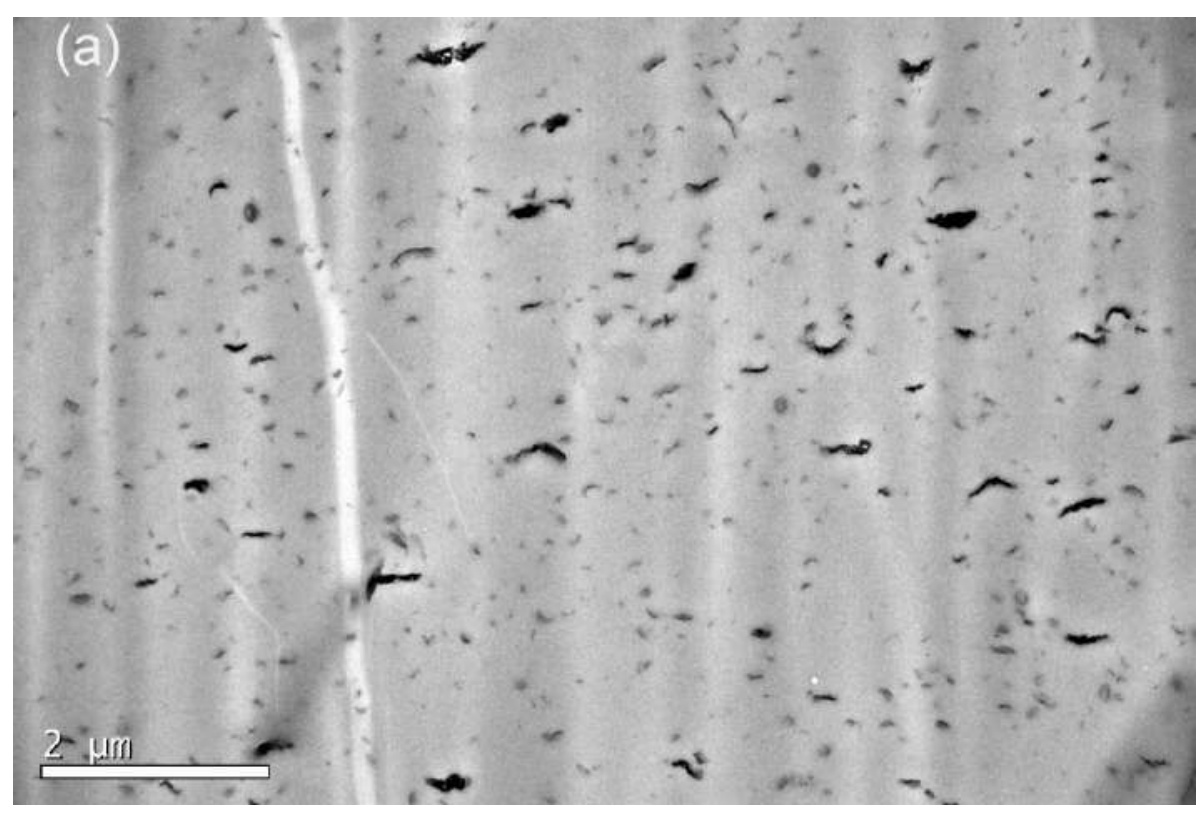




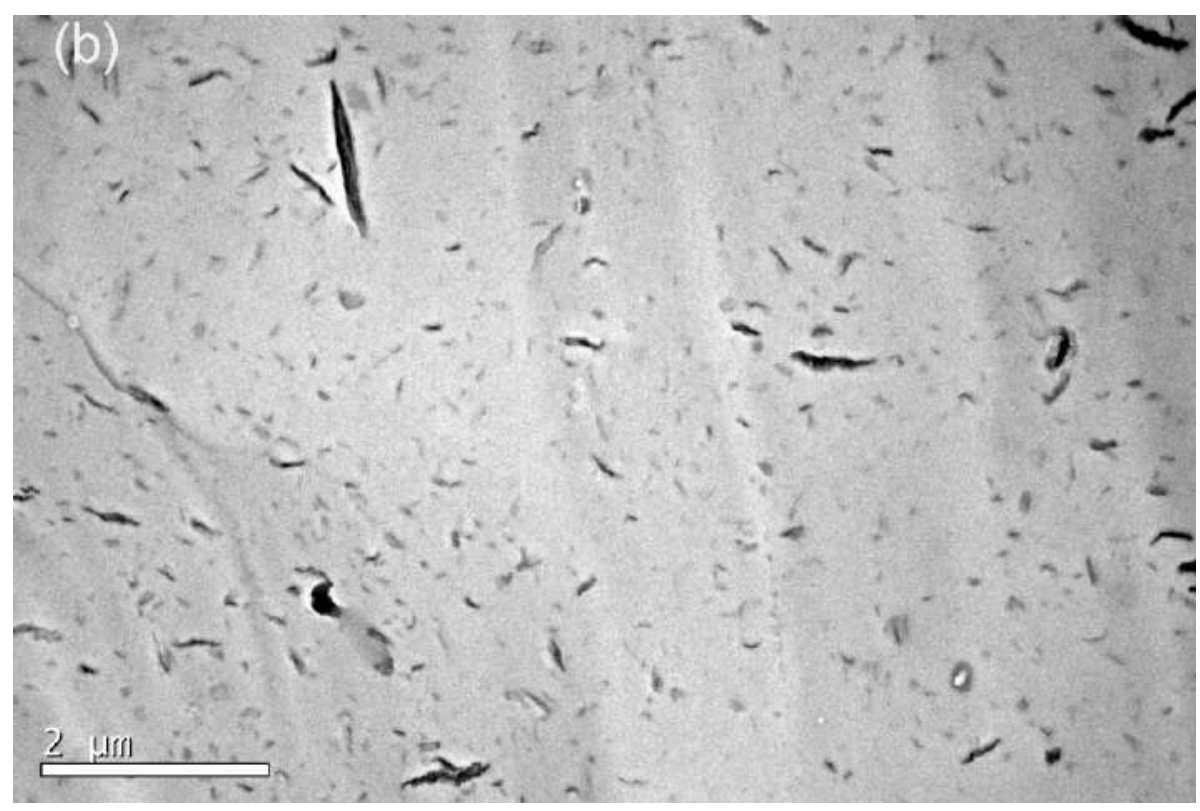

Fig. 8. Fotografías TEM del PLAV-C30 (a) y PLAR-C30 (b).

Además de las fotografías de TEM, se realizaron ensayos de XRD en los nanocomposites con C30 para obtener más información de los cambios observados en la morfología de estos materiales después de ser sometidos a los diferentes procesos de reciclado. Los difractogramas tanto de la arcilla C30, como de los diferentes nanocomposites (en la región $2 \theta<8^{\circ}$ ) son presentados en la Fig. 9. En el difractograma que corresponde a la arcilla C30, se puede observar el pico de reflexión (001) ubicado a $2 \theta=4,8^{\circ}$, que corresponde (de acuerdo a la Ley de Bragg) a un espaciado basal $\mathrm{d}_{001}=1,8$ $\mathrm{nm}$. En el nanocomposite del material virgen (PLAV-C30) este pico se encuentra desplazado hacia menores ángulos, concretamente hacia $2 \theta=2,6^{\circ}$, que corresponde a un espaciado basal $\mathrm{d}_{001}=3,4 \mathrm{~nm}$. Este incremento de la distancia entre las galerías de arcilla es consecuencia de la entrada de las cadenas poliméricas en la arcilla, dando lugar a una estructura intercalada. El PLAV-C30 muestra también un segundo pico, más ancho, a $2 \theta=$ $5,4^{\circ}$ que podría ser atribuido, al menos en parte, a la reflexión de segundo orden de la difracción principal. Además, también puede contribuir a este pico la formación de agregados de arcilla con menor espaciado basal, la cual fue observada en las fotografías TEM de la muestra. En la literatura se encuentran resultados similares, como los reportados por Pluta et al. [94], quienes encontraron el colapso de parte de la arcilla en nanocomposites de PLA con 3 y 10\% de C30. 
Capítulo 4. Efecto del reciclado en estructura y propiedades

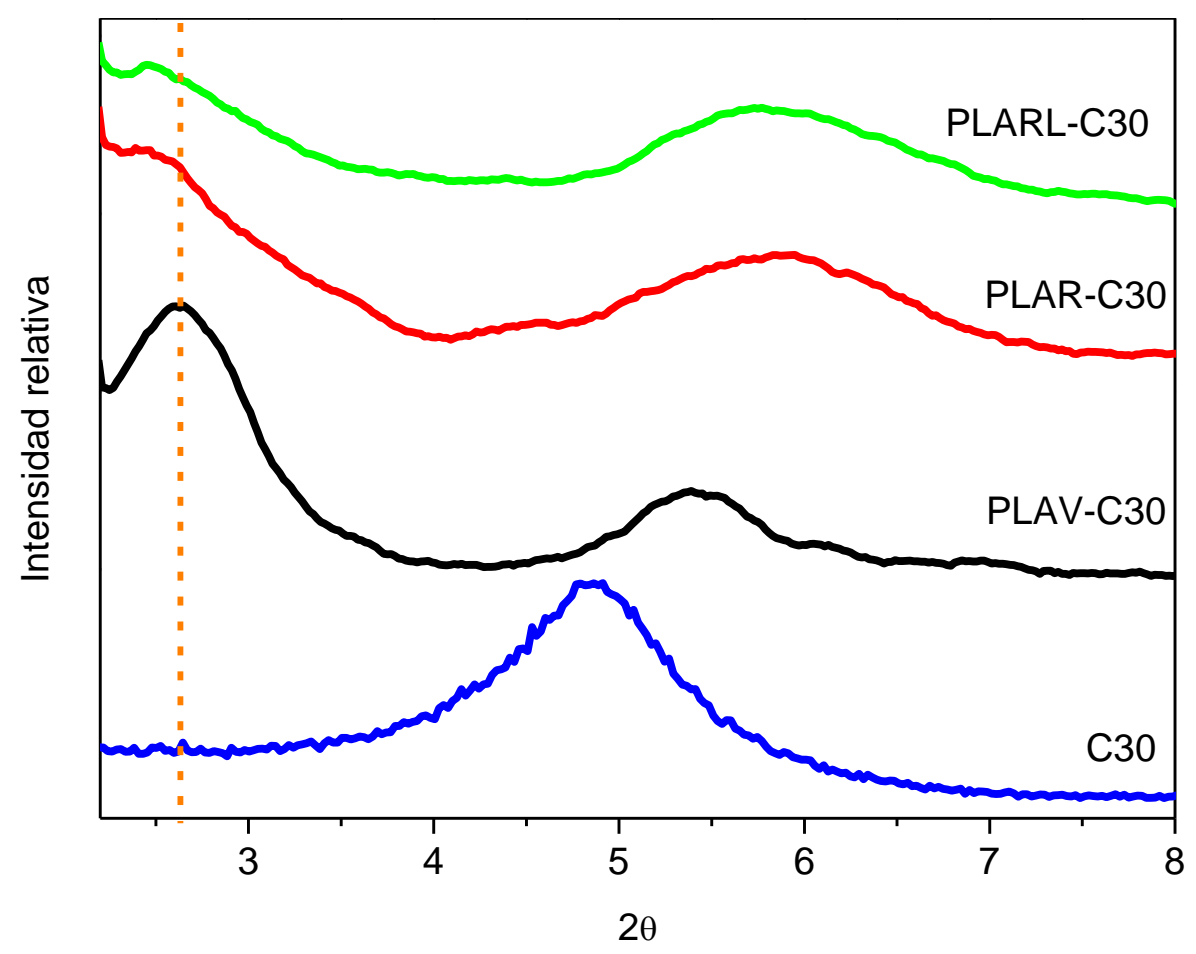

Fig. 9. Difractogramas de la arcilla C30, y de los nanocomposites PLA-C30 virgen y reciclados $\left(2 \theta<8^{\circ}\right)$.

En cuanto al efecto del reciclado en la dispersión de la arcilla en los nanocomposites, la Fig. 9 muestra que el pico de reflexión (001) de la arcilla prácticamente desaparece en el PLAR-C30 y en el PLARL-C30. Además, la débil difracción (001) resultante es desplazada hacía ángulos más bajos, lo que concuerda con los resultados observados mediante TEM. Estos resultados indican una mejora en la dispersión de la arcilla durante el reciclado mecánico, llegándose a formar estructuras exfoliadas, en las que las láminas de arcillas están muy separadas y son incapaces de interactuar entre ellas.

También se han analizado mediante TEM los nanocomposites PLA-haloisita, para estudiar el efecto del reciclado en la dispersión de la arcilla. Las imágenes se presentan en la Fig. 10. Al igual que en el caso de los nanocomposites con C30, en los materiales con haloisita se observa una mejora en la dispersión de los nanotubos de arcilla como consecuencia del reciclado mecánico. Si se comparan las micrografías TEM de los nanocomposites con $\mathrm{C} 30 \mathrm{y}$ con haloisita, parece que los primeros presentan una mejor dispersión de la arcilla. Este comportamiento podría ser debido a que la C30 es una arcilla modificada orgánicamente, por lo que su compatibilidad con el PLA es mayor que la de la haloisita sin modificar. 

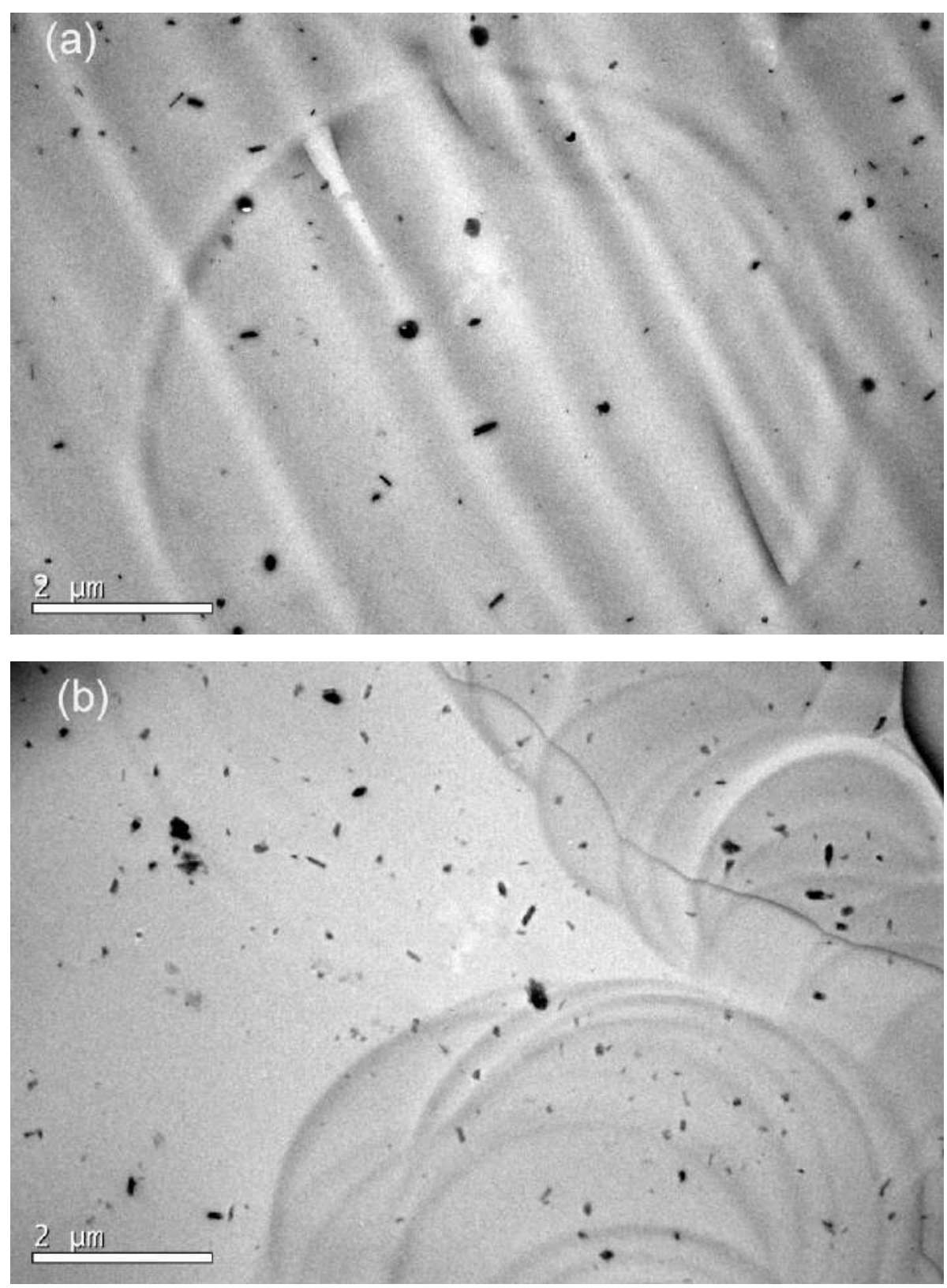

Fig. 10. Fotografías TEM del PLAV-Ha (a) y PLAR-Ha (b).

En resumen, los resultados de TEM y XRD indican una mejora en el grado de dispersión de la arcilla, tanto la C30 como la haloisita, después del reciclado mecánico. Dicha mejora está relacionada con un mayor tiempo de residencia en la extrusora y con la degradación del PLA, como consecuencia de la etapa de reprocesado. Esta variación en el grado de dispersión de las arcillas podría afectar las propiedades térmicas, mecánicas, ópticas y de barrera de los materiales reciclados.

\subsection{Estructura de los materiales vírgenes y reciclados}


Una vez analizados los cambios en el peso molecular del PLA, y en la morfología de los nanocomposites, como consecuencia de los diferentes procesos de reciclado mecánico, se analizaron los cambios en la estructura de los diferentes materiales mediante espectroscopía FTIR-ATR, DSC y XRD.

En la Fig. 11 se presentan los espectros del PLA virgen y sometido a diferentes procesos de reciclado mecánico. En primer lugar, se puede apreciar que en todos los casos se observan las bandas de absorción características del PLA, las cuales se asignan en la Tabla $3[49,59]$.

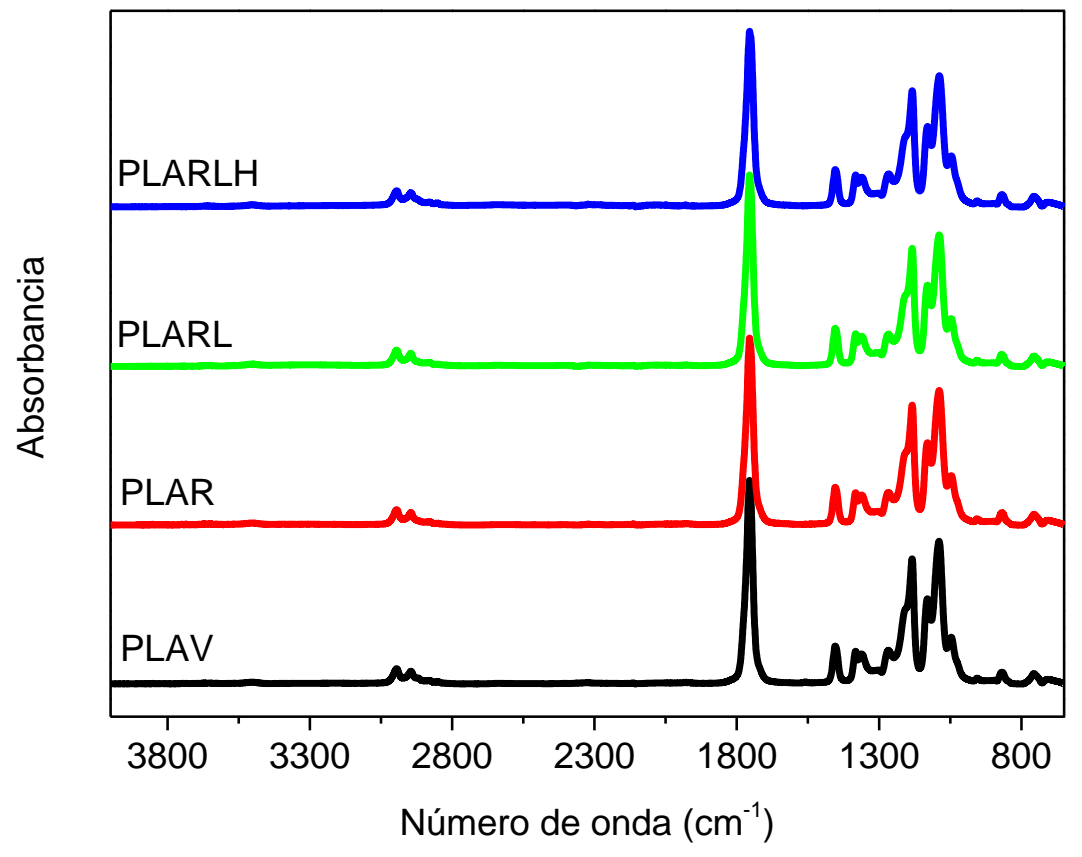

Fig. 11. Espectros FTIR del PLA virgen y sometido a diferentes procesos de reciclado.

Tabla 3. Bandas de absorción características del PLA.

\begin{tabular}{cc}
\hline Número de onda $\left(\mathbf{c m}^{-\mathbf{1}}\right)$ & Grupo asociado \\
\hline 2995 & Tensión asimétrica $\mathrm{CH}_{3}$ \\
2945 & Tensión simétrica $\mathrm{CH}_{3}$ \\
1755 & Tensión $\mathrm{C}=\mathrm{O}$ \\
1452 & Flexión asimétrica $\mathrm{CH}_{3}$ \\
1383 & Flexión simétrica $\mathrm{CH}_{3}$ \\
1360 & Flexión $\mathrm{C}-\mathrm{H}$ \\
1268 & Flexión $\mathrm{C}-\mathrm{H}$ y tensión $\mathrm{C}-\mathrm{O}-\mathrm{C}$ \\
$1212-1185$ & Tensión asimétrica C-O-C y balanceo $\mathrm{CH} 3$ \\
1130 & Balanceo asimétrico $\mathrm{CH}$ \\
1090 & Tensión simétrica $\mathrm{C}-\mathrm{O}-\mathrm{C}$ \\
1045 & Tensión C- $\mathrm{CH}$ \\
$956-920$ & Balanceo $\mathrm{CH}_{3} \mathrm{y}$ tensión $\mathrm{C}-\mathrm{C}$ \\
\hline
\end{tabular}


La espectroscopía FTIR también puede usarse como una herramienta para estudiar la degradación del PLA durante el reciclado mecánico. Por ejemplo, Badía et al. [14] reportaron cambios en dos regiones concretas del espectro en el PLA reprocesado: (i) un incremento en la absorbancia de las bandas localizadas a 2995 y $2945 \mathrm{~cm}^{-1}$ y (ii) un incremento, y desplazamiento, de la banda de tensión del grupo carbonilo (a $1755 \mathrm{~cm}^{-1}$ ). Dichos cambios se atribuyeron a la generación de nuevos compuestos con enlaces $\mathrm{C}=\mathrm{O}$, tanto en posiciones medias como en los extremos de las cadenas de PLA, y que son un síntoma de la reducción del peso molecular. En la Fig. 12 se puede observar que el PLARLH presenta un pequeño desplazamiento hacia menores números de onda de la banda $\mathrm{C}=\mathrm{O}$, lo que sugiere la presencia de grupos carboxilos, debida a la degradación del PLA durante el reciclado.

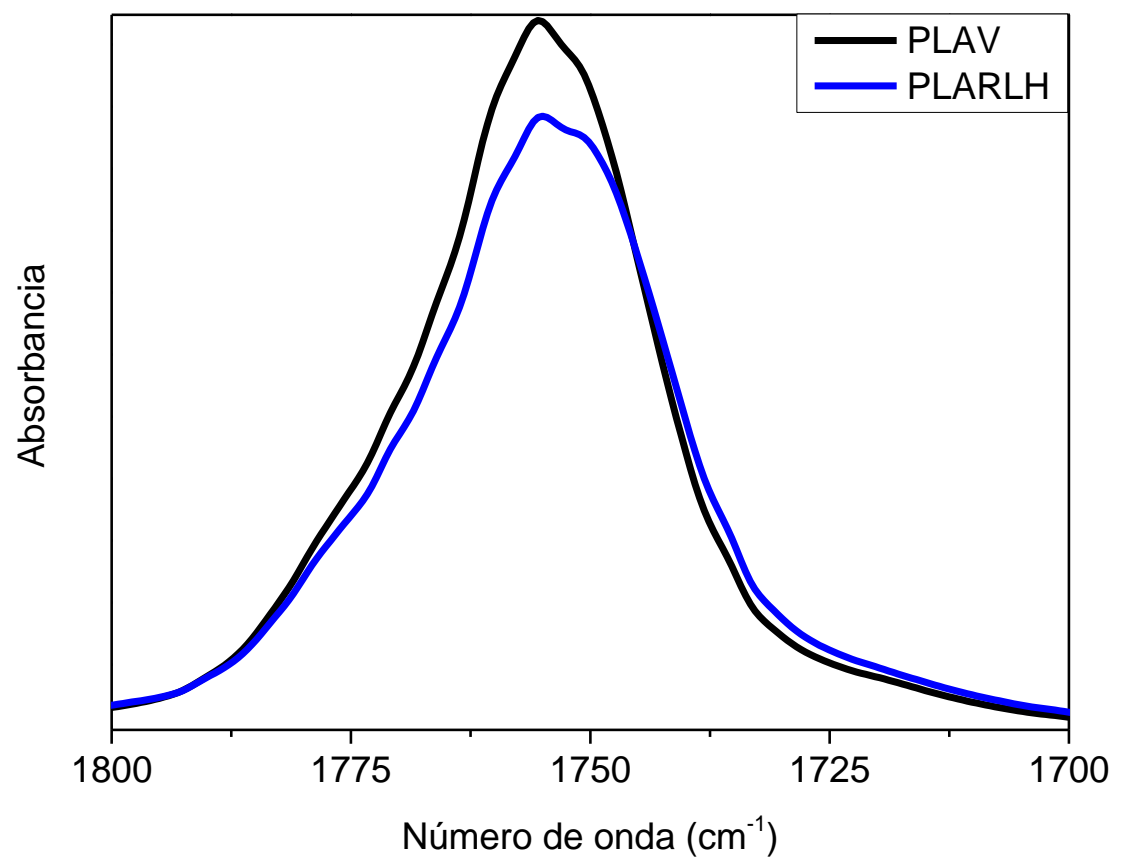

Fig. 12. Ampliación de la banda de absorción centrada a $1755 \mathrm{~cm}^{-1}$ del PLAV y PLARLH.

En cuanto al estudio de la estructura de los nanocomposites, la Fig. 13 muestra que el comportamiento observado es similar al del PLA puro, por lo que la presencia de pequeñas cantidades de arcilla no afecta en gran medida los espectros FTIR del PLA. 


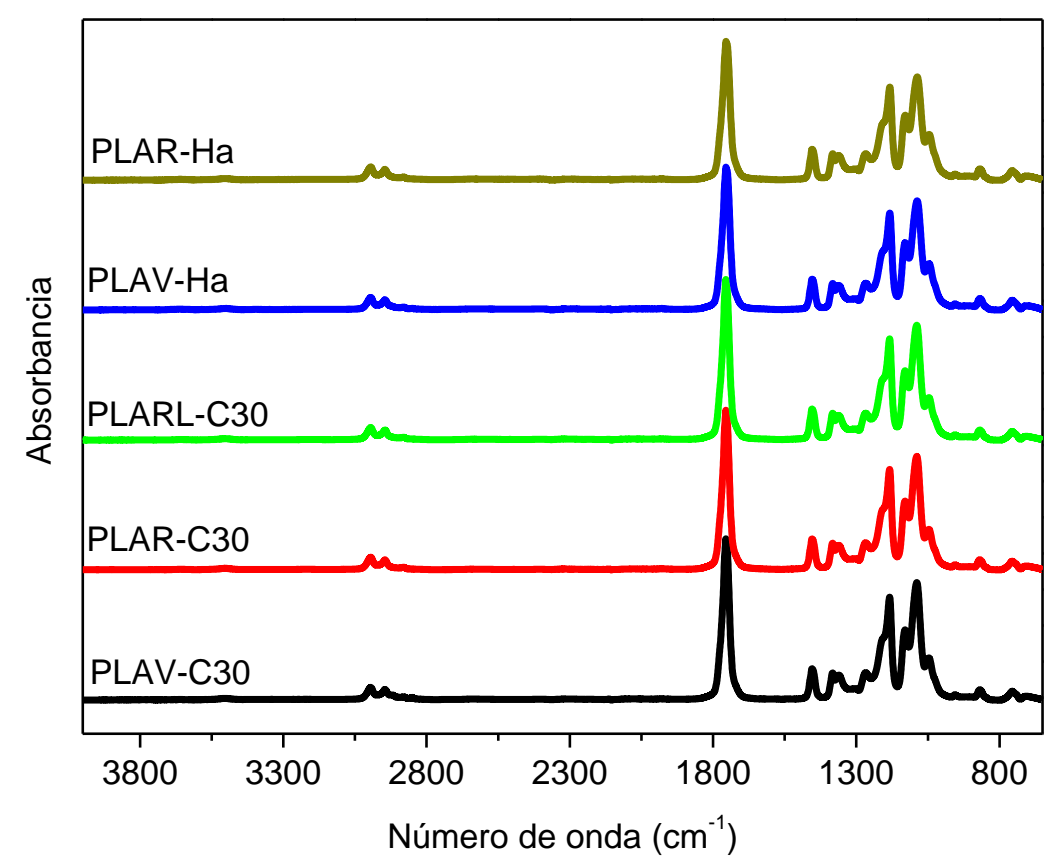

Fig. 13. Espectro FTIR de los nanocomposites de PLA sometidos a diferentes procesos de reciclado.

Además de los ensayos FTIR, se emplearon otras técnicas para estudiar los cambios estructurales del PLA y sus nanocomposites durante el reciclado. Los ensayos de XRD también fueron utilizados para analizar los cambios estructura cristalina del PLA durante los diferentes procesos de reciclado. En la Fig. 14 se muestran los difractogramas del PLA puro sometido a diferentes procesos de reciclado. Se puede observar que todas las muestras analizadas presentan un pico ancho, centrado alrededor de $16,7^{\circ}$, que es característico del PLLA amorfo [20]. Este resultado sugiere que, a pesar de la degradación del PLA, los diferentes procesos de reciclado no ocasionan grandes cambios en la naturaleza amorfa del PLA. 


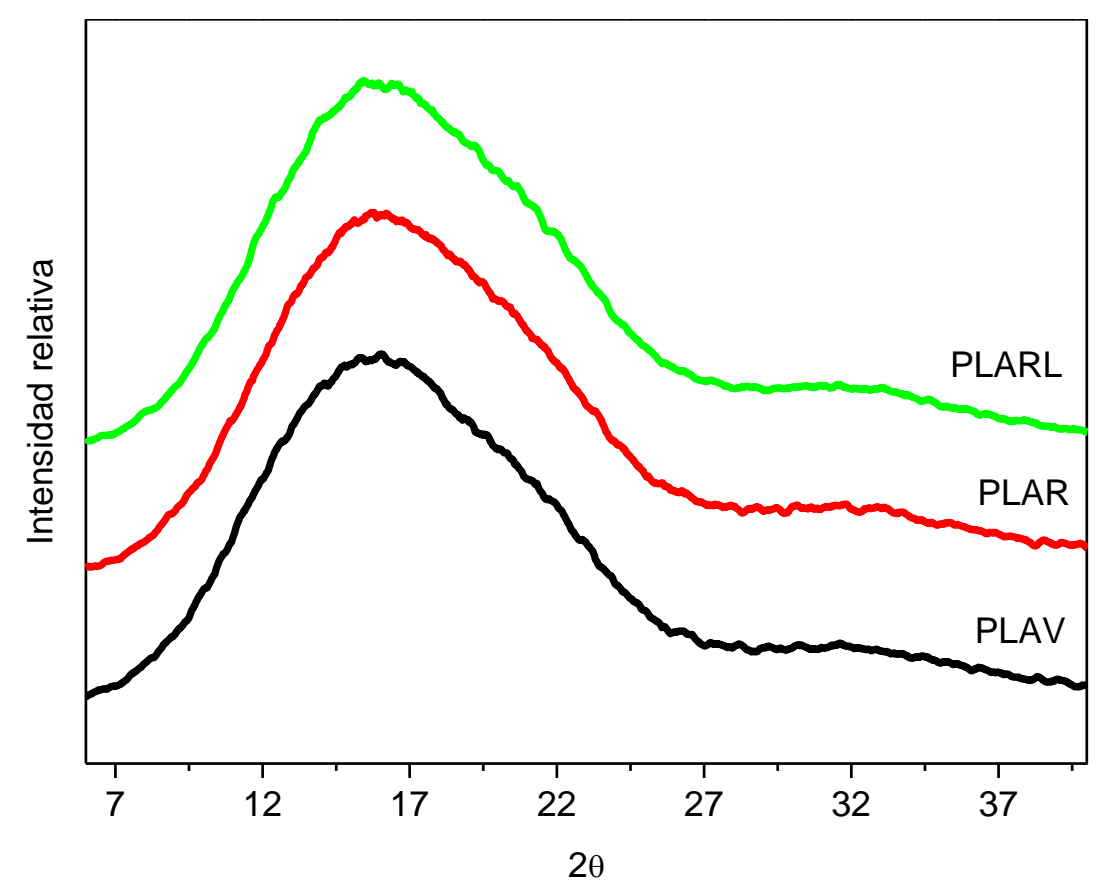

Fig. 14. Difractogramas del PLAV, PLAR y PLARL $\left(6^{\circ}<2 \theta<40^{\circ}\right)$.

Los nanocomposites PLA-C30, cuyos difractogramas se muestran en la Fig. 15, muestran un comportamiento similar al del PLA puro, ya que sólo se observa el pico correspondiente al PLA amorfo en los nanocomposites virgen y reciclado. Este resultado es importante, ya que la estructura cristalina influye en gran medida en las propiedades ópticas, mecánicas y de barrera del PLA y sus nanocomposites.

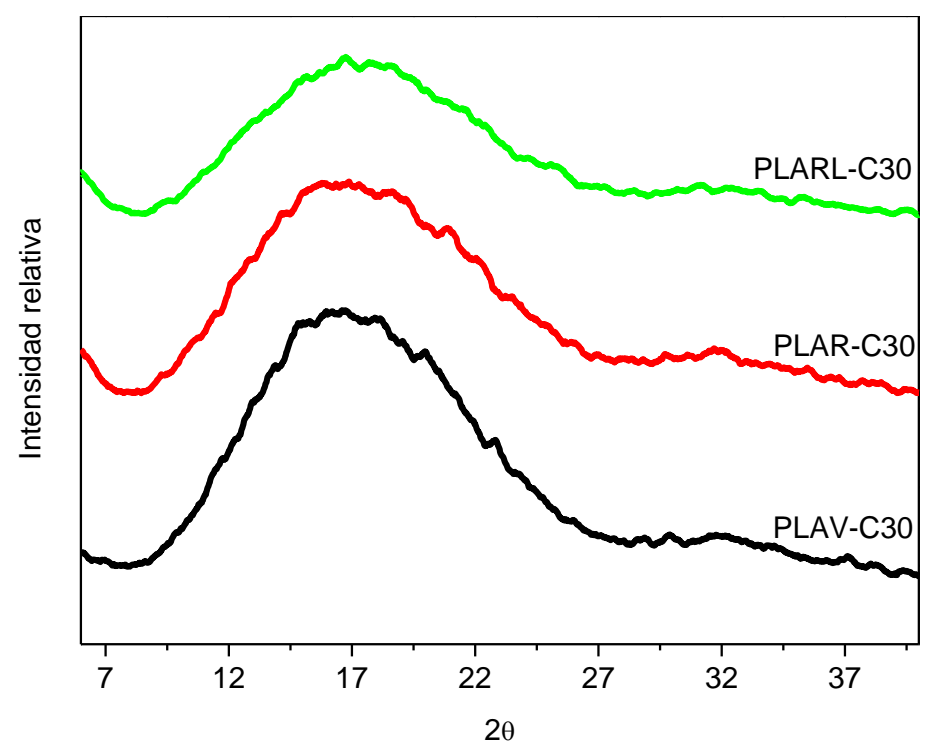

Fig. 15. Difractogramas del nanocomposite PLA-C30 sometido a diferentes procesos de reciclado mecánico $\left(6^{\circ}<2 \theta<40^{\circ}\right)$. 
Con la finalidad de complementar los resultados obtenidos mediante XRD, se realizaron ensayos de DSC a todos los materiales vírgenes y reciclados, cuyos resultados se encuentran resumidos en la Tabla 4.

En primer lugar, en la Fig. 16 se presentan los barridos correspondientes al segundo calentamiento del PLA puro, sometido a diferentes procesos de reciclado. Se puede observar que los barridos de todas las muestras son similares, mostrando una transición vítrea alrededor de $60^{\circ} \mathrm{C}$, una cristalización en frío entre 100 y $107^{\circ} \mathrm{C}$ y una endoterma de fusión por encima de $140{ }^{\circ} \mathrm{C}$. Un aspecto importante sobre las muestras estudiadas es la presencia de un doble pico de fusión que, según diferentes autores, puede ser atribuido a un mecanismo de recristalización en fundido. Este mecanismo consiste en la fusión a temperaturas bajas de los cristales menos perfectos, su reorganización en estructuras más ordenadas, y su posterior fusión a una temperatura mayor, tal y como reportan Yasuniwa et al. [115], Ling y Spruiell [67] y Di Lorenzo [44].

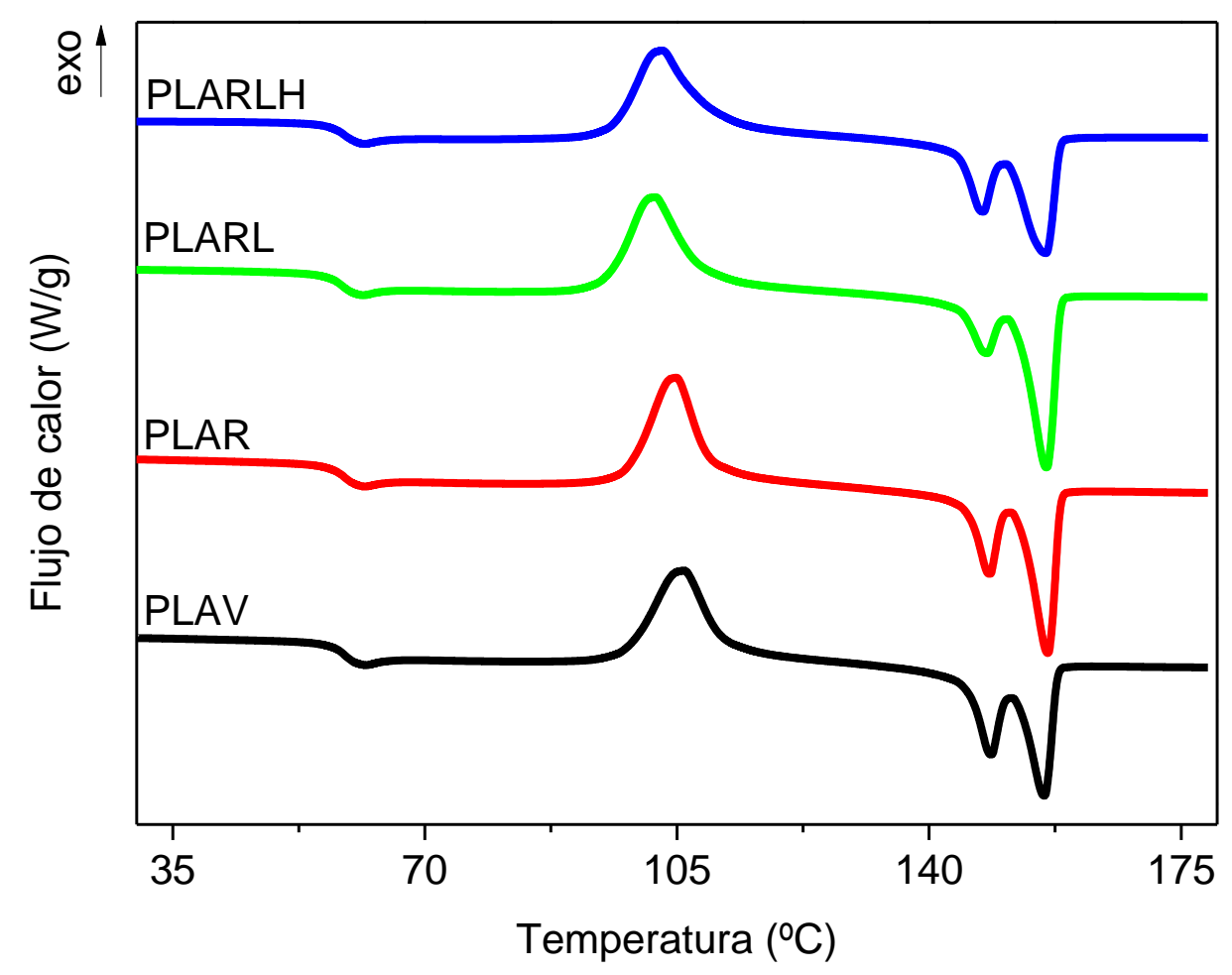

Fig. 16. Barridos del segundo calentamiento del PLA sometido a diferentes procesos de reciclado.

En cuanto al efecto del reciclado, en la Fig. 16 se pueden observar pequeñas diferencias tanto en la cristalización en frío como en la fusión del polímero. En primer lugar, los materiales reciclados presentan una temperatura de cristalización ligeramente menor que el PLAV. Este cambio puede explicarse si se toma en cuenta la degradación 
del PLA durante el reciclado mecánico, que ha sido observada en las medidas de viscosidad intrínseca, ya que las cadenas más cortas de los materiales reciclados tienen una mayor movilidad, por lo que cristalizan a una temperatura menor.

En segundo lugar, el pico de fusión de alta temperatura parece tener una mayor relevancia en los materiales reciclados que en el material virgen. Este cambio también podría estar relacionado con la menor longitud de cadena de los materiales reciclados, que permitirían un mejor reordenamiento durante la recristalización en fundido, formando así un mayor número de cristales que fundan a mayor temperatura.

En lo que respecta al efecto de los diferentes procesos de reciclado en la cristalinidad del PLA, en la Tabla 4 se puede observar que los valores de las entalpías de cristalización y fusión se encuentran muy próximos, lo que indica que los cristales fundidos son aquellos formados durante la cristalización en frío, y que por tanto la naturaleza amorfa del PLA no se ve afectada por los diferentes procesos de reciclado mecánico.

Tabla 4. Resultados de DSC (segundo barrido de calentamiento) de los diferentes materiales.

\begin{tabular}{cccccc}
\hline Material & $\begin{array}{c}\mathbf{T}_{\mathbf{g}} \\
\left({ }^{\mathbf{o}} \mathbf{C}\right)\end{array}$ & $\begin{array}{c}\mathbf{T}_{\mathbf{c c}} \\
\left({ }^{\mathbf{o}} \mathbf{C}\right)\end{array}$ & $\begin{array}{c}\mathbf{T}_{\text {fus }} \\
\left({ }^{\mathbf{o}} \mathbf{C}\right)\end{array}$ & $\begin{array}{c}\Delta \mathbf{H}_{\text {crist }} \\
(\mathbf{J} / \mathbf{g})\end{array}$ & $\begin{array}{c}\Delta \mathbf{H}_{\text {fus }} \\
(\mathbf{J} / \mathbf{g})\end{array}$ \\
\hline PLAV & 58,9 & 106,0 & $148,6-156,0$ & 27,5 & 27,6 \\
PLAR & 58,8 & 105,0 & $148,3-156,5$ & 29,5 & 28,3 \\
PLARL & 58,5 & 102,0 & $148,1-156,3$ & 28,9 & 28,8 \\
PLARLH & 58,8 & 103,0 & $147,6-156,3$ & 27,4 & 28,3 \\
\hline PLAV-C30 & 58,9 & 106,1 & $148,7-156,3$ & 27,5 & 28,5 \\
PLAR-C30 & 59.3 & 102.0 & $148,0-156,0$ & 27,3 & 27,7 \\
PLARL-C30 & 58,4 & 100,9 & $147,7-156,5$ & 29,1 & 30,0 \\
\hline PLAV-Ha & 58,6 & 107,3 & $149,0-156,4$ & 27,9 & 28,0 \\
PLAR-Ha & 59,0 & 102,6 & $148,0-156,2$ & 27,6 & 27,8 \\
\hline
\end{tabular}

En cuanto al efecto del reciclado en los nanocompuestos PLA-C30 y PLA-Ha, la Tabla 4, la Fig. 17 y la Fig. 18 muestran que el comportamiento es similar al observado en el PLA puro, observándose una disminución en la temperatura de cristalización en frío y un incremento de la relevancia del pico de fusión de mayor temperatura durante el reciclado, mientras que la naturaleza amorfa del PLA no se ve afectada. 
Capítulo 4. Efecto del reciclado en estructura y propiedades

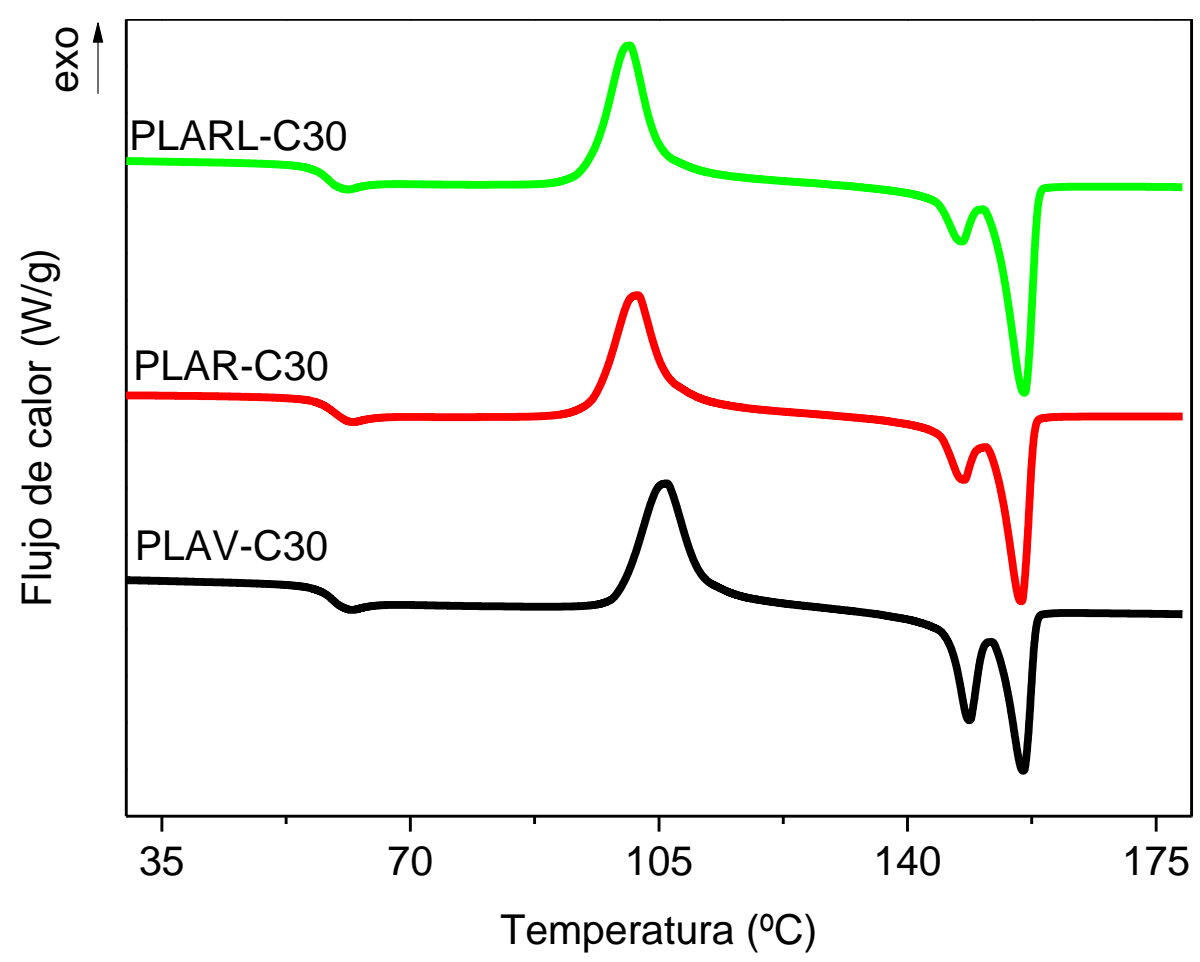

Fig. 17. Barridos del segundo calentamiento del nanocomposite PLA-C30 sometido a diferentes procesos de reciclado.

Los resultados de DSC indican que, en general, los cambios estructurales en el PLA, y sus nanocompuestos, durante el reciclado son pequeños, limitándose a un descenso en la temperatura de cristalización en frío, y al aumento en la relevancia del pico de fusión de alta temperatura. En cambio, la naturaleza amorfa del PLA parece no ser afectada por el reciclado. 


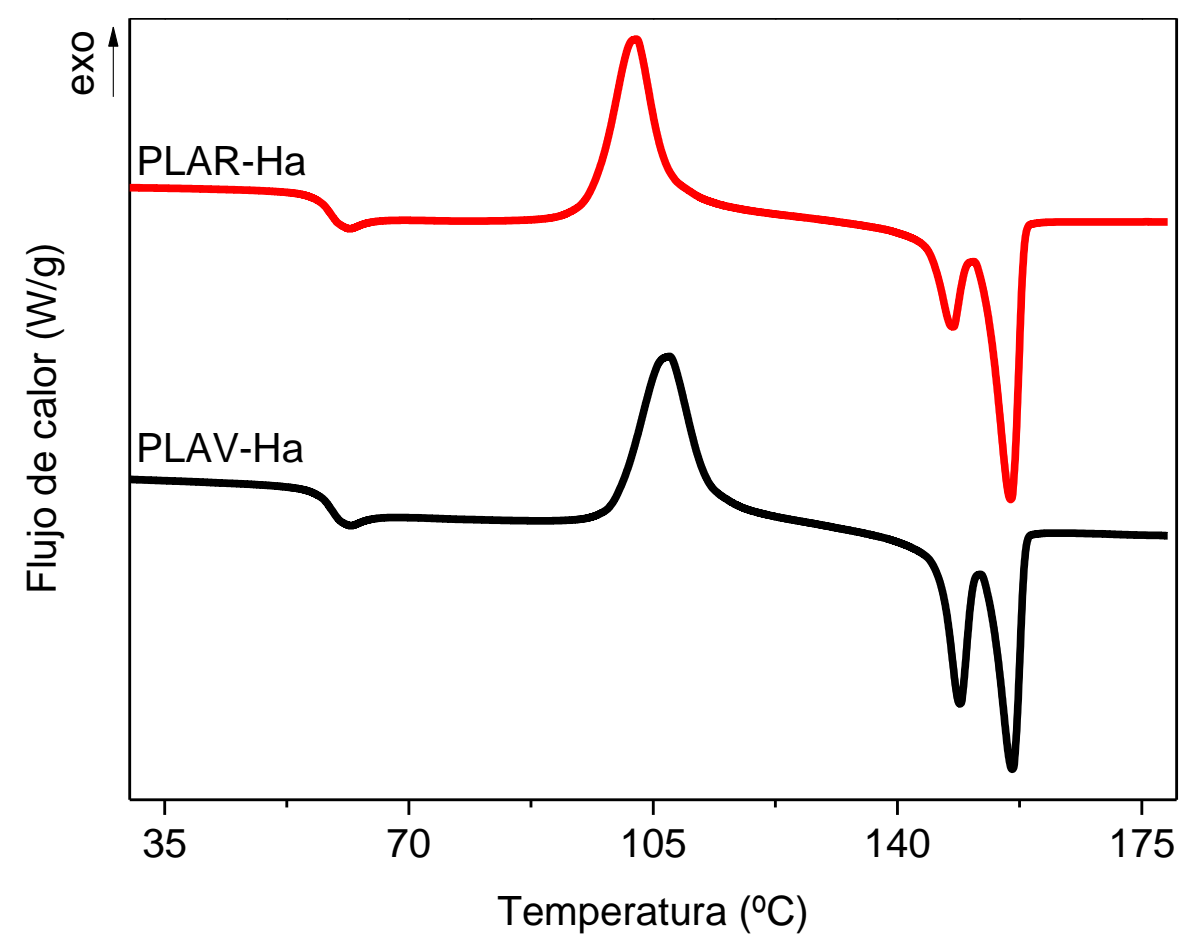

Fig. 18. Barridos del segundo calentamiento del nanocomposite PLA-Ha sometido a diferentes procesos de reciclado.

\subsection{Estabilidad térmica de los materiales vírgenes y reciclados}

Los cambios observados en la viscosidad intrínseca, morfología y estructura durante el reciclado mecánico del PLA y sus nanocomposites podrían afectar diferentes propiedades de los materiales, influyendo negativamente en su reciclabilidad. A continuación, se estudia la estabilidad térmica de los diferentes materiales, a partir de los resultados obtenidos de los ensayos TGA. La Fig. 19 muestra los termogramas del PLA puro sometido a diferentes procesos de reciclado mecánico. Se puede observar que, de forma similar a lo observado en las medidas de viscosidad intrínseca, el reciclado mecánico ocasiona una ligera disminución de la estabilidad térmica del PLA. Dicho descenso en la estabilidad es mayor en las muestras sometidas al proceso de lavado. Este comportamiento se debe a la degradación del PLA durante los diferentes procesos de reciclado, ya que las cadenas más cortas se descomponen a una temperatura menor, tal y como señalan Carrasco et al. [27] en su estudio sobre el procesado de PLA y su influencia en la estabilidad térmica. Sin embargo, en la Tabla 5 se puede observar que la disminución en $\mathrm{T}_{10}$ y $\mathrm{T}_{\max }$ (temperaturas donde se ha perdido el $10 \%$ de masa y de degradación máxima, respectivamente), que usualmente se utilizan para como indicadores de estabilidad térmica, es relativamente pequeña, sobre todo si se compara con las disminuciones de la viscosidad intrínseca. Este resultado sugiere que, además de la degradación del PLA, podrían existir otros factores que afecten la estabilidad térmica de los materiales reciclados. Por ejemplo, durante la degradación termomecánica del PLA se generan compuestos con grupos carboxilos, que podrían incrementar las interacciones 
entre las cadenas, lo que contrarrestaría el efecto negativo de la degradación en la estabilidad térmica.

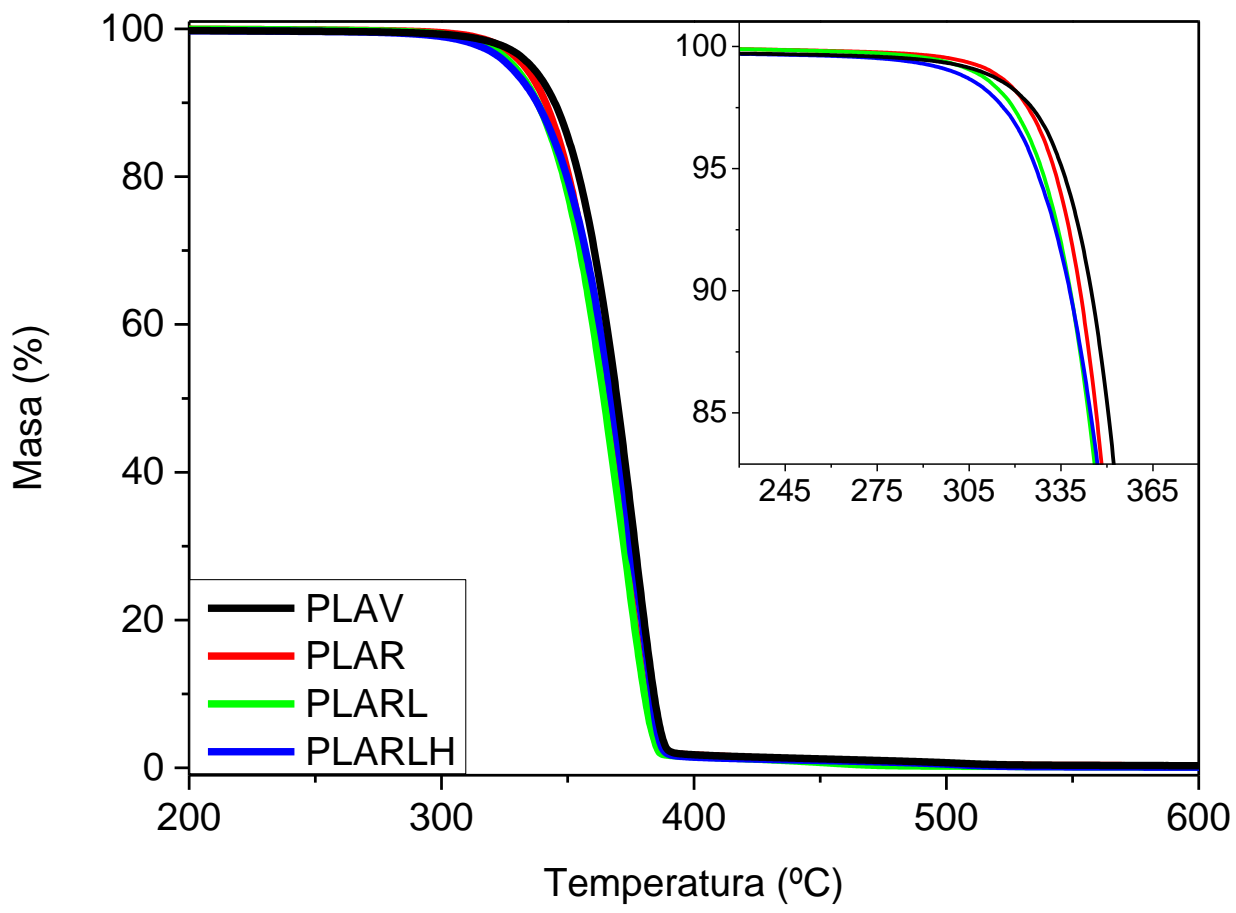

Fig. 19. Termogramas del PLA sometido a diferentes procesos de reciclado mecánico.

En el caso de los nanocomposites PLA-C30, se puede observar en la Fig. 20 que los termogramas de todos los materiales son muy parecidos. Sin embargo, si se observan los valores de $\mathrm{T}_{\max }$ reportados en la Tabla 5 se puede ver como los nanocomposites reciclados presentan una mayor $\mathrm{T}_{\max }$. Estos resultados señalan que, a pesar de la degradación del PLA durante el reciclado, la estabilidad térmica de los nanocomposites reciclados aumenta ligeramente. Este resultado podría ser explicado por la generación de grupos carboxilo durante el reciclado (al igual que en el PLA puro), sin embargo, a este factor habría que añadir la mejora en la dispersión de las láminas de arcilla en la matriz de PLA, que fue observada mediante TEM y ensayos de XRD. Las láminas de arcilla dispersas en la matriz polimérica actúan como una barrera para la liberación de los productos de la descomposición del PLA, retrasando de esta forma la pérdida de masa. Un argumento similar fue expuesto por Paul et al. [89] al estudiar nanocomposites de PLA y diferentes cantidades de montmorillonita, tanto natural como orgánicamente modificada. 


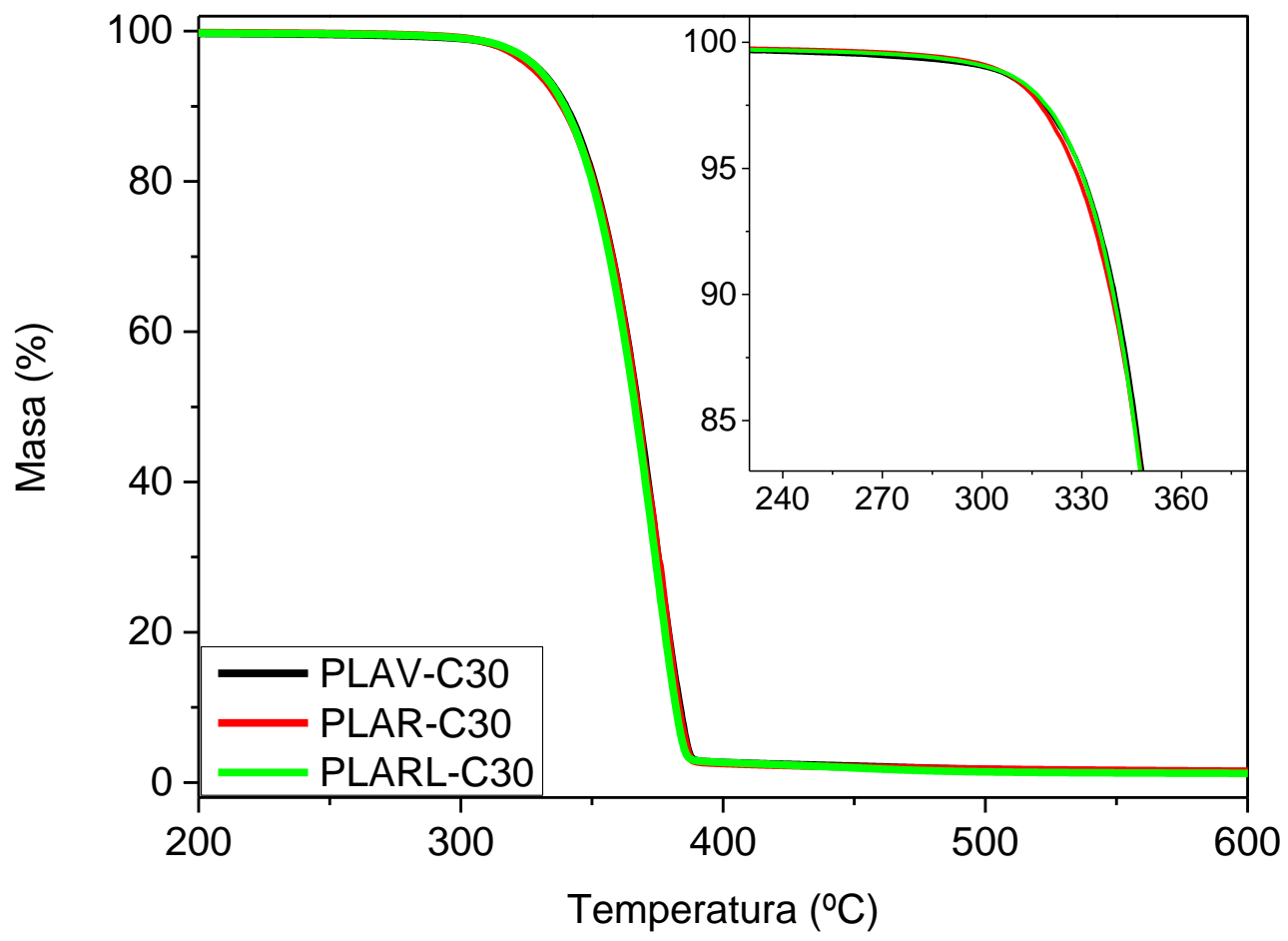

Fig. 20. Termogramas de los nanocomposites PLA-C30 sometido a diferentes procesos de reciclado mecánico.

La contraposición de los efectos de la degradación del PLA, la mejora en la dispersión de las arcillas y la generación de compuestos con grupos carboxilos también puede observarse en los nanocomposites con haloisita, ya que los valores de $\mathrm{T}_{10}$ y $\mathrm{T}_{\max }$ reportados en la Tabla 5 son bastante parecidos para el PLAV-Ha y el PLAR-Ha.

En resumen, durante el reciclado mecánico del PLA y sus nanocomposites tienen lugar diferentes procesos con efectos contrapuestos en la estabilidad térmica del material: la degradación del polímero, la generación de compuestos con grupos carboxílicos y la mejora en la dispersión de las arcillas. La presencia de estos procesos conlleva a que el efecto global del reciclado mecánico sobre la estabilidad térmica del PLA y sus nanocomposites sea pequeño.

Tabla 5. Temperaturas características de TGA de los diferentes materiales.

\begin{tabular}{ccc}
\hline Muestra & $\mathbf{T}_{\mathbf{1 0}}\left({ }^{\mathbf{0}} \mathbf{C}\right)$ & $\mathbf{T}_{\mathbf{m a x}}\left({ }^{\mathbf{0}} \mathbf{C}\right)$ \\
\hline PLAV & 344,9 & 376,4 \\
PLAR & 341,1 & 371,7 \\
PLARL & 338,1 & 372,6 \\
PLARLH & 337,9 & 372,3 \\
\hline PLAV-C30 & 340,0 & 367,3 \\
PLAR-C30 & 338,7 & 377,7 \\
PLARL-C30 & 339,4 & 374,1 \\
\hline
\end{tabular}




\begin{tabular}{lll}
\hline PLAV-Ha & 342,1 & 372,1 \\
PLAR-Ha & 342,6 & 371,8 \\
\hline
\end{tabular}

\subsection{Propiedades ópticas de los materiales reciclados}

El PLA utilizado en este trabajo está diseñado especialmente para aplicaciones de envasado. En este campo, las propiedades ópticas como la transmisión de luz son muy importantes. En este trabajo, se han realizado ensayos de espectroscopía UV-Vis de los diferentes materiales, con la finalidad de determinar el efecto del reciclado mecánico en las propiedades ópticas tanto del PLA como de sus nanocomposites reciclados.

En la Fig. 21 se presentan los espectros UV-Vis del PLA puro sometido a diferentes procesos de reciclado mecánico. Para estudiar mejor el efecto del reciclado es conveniente dividir el espectro en dos partes: la región UV (por debajo de $400 \mathrm{~nm}$ ) y la región visible (entre 400 y $800 \mathrm{~nm}$ ). En la región UV se puede observar que los materiales reciclados, especialmente aquellos sometidos a una etapa de lavado enérgico, presentan una banda de absorción alrededor de $275 \mathrm{~nm}$. Esta banda podría estar relacionada con la presencia de grupos terminales carboxilos, como consecuencia de la degradación del PLA durante el reciclado [32]. De hecho, en un estudio realizado por Badía et al. [15] se ha reportado que el reprocesado de PLA puede ocasionar un cambio en la distribución de los oligómeros en el polímero, con un aumento de los grupos terminales carboxilo. Cabe mencionar que el aumento en la absorción observado en las muestras sometidas a la etapa de lavado coincide con el comportamiento observado en las medidas de viscosidad intrínseca, donde el PLARL y el PLARLH presentaban una mayor degradación que el PLAR.

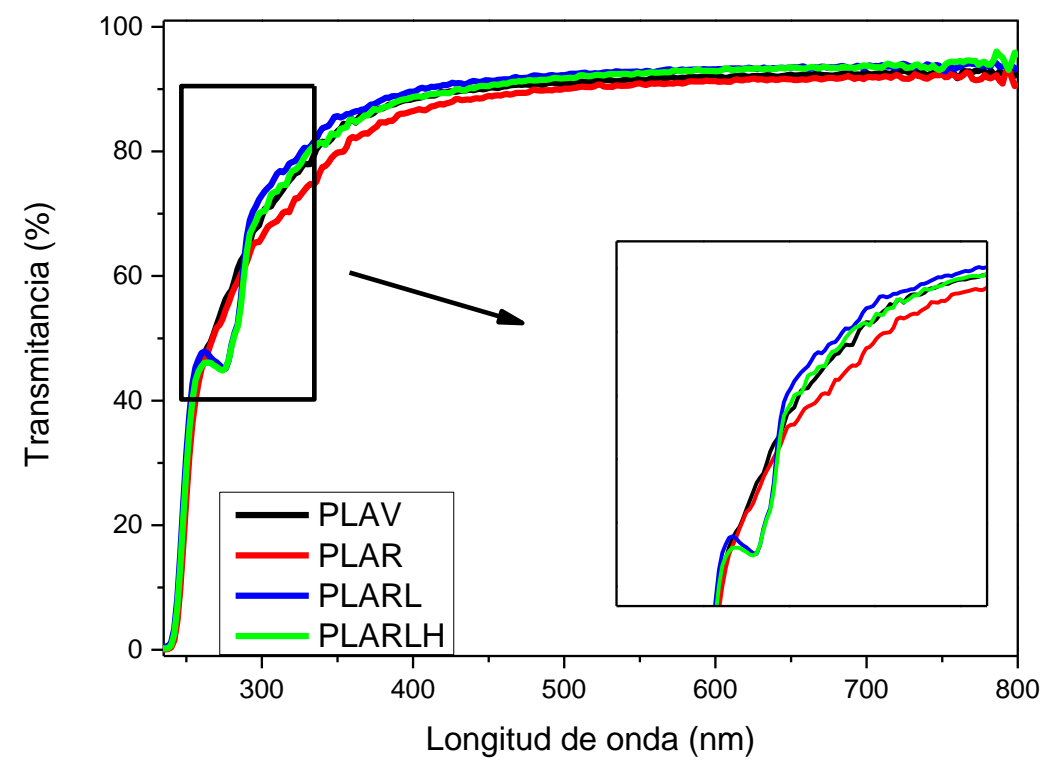

Fig. 21. Espectros UV-Vis del PLA sometido a diferentes procesos de reciclado mecánico. 
En cuanto al comportamiento de los materiales reciclados en la región visible del espectro, se puede observar en la Fig. 21 que todos los materiales se comportan de una manera similar. De hecho, en la Tabla 6 se presentan los valores de transmisión de luz de las diferentes muestras, y se puede apreciar que el PLAV, PLAR, PLARL y PLARLH presentan valores cercanos al 90\%. Este comportamiento puede ser explicado si se considera la naturaleza amorfa de todas las muestras, tal y como se ha observado en los ensayos de XRD y DSC, ya que no hay estructuras cristalinas que pudiesen actuar como centros difusores o reflectores de luz en ninguno de los materiales.

Tabla 6. Transmisión de luz de los materiales vírgenes y reciclados.

\begin{tabular}{cc}
\hline Muestra & Transmisión de luz (\%) \\
\hline PLAV & 90,1 \\
PLAR & 88,7 \\
PLARL & 90,8 \\
PLARLH & 90,5 \\
\hline PLAV-C30 & 87,6 \\
PLAR-C30 & 89,0 \\
PLARL-C30 & 86,9 \\
\hline PLAV-Ha & 87,3 \\
PLAR-Ha & 87,1 \\
\hline
\end{tabular}

La Fig. 22 muestra los espectros UV-Vis de los nanocomposites PLA-C30, virgen y reciclado. Por una parte, en la región UV no es posible percibir la banda de absorción alrededor de $275 \mathrm{~nm}$ que se observa en el PLA puro. Este comportamiento se debe a que los nanocomposites PLA-C30 presentan una menor transmisión en la región UV que el PLA puro, lo que impide observar la banda atribuida a los grupos carboxilos. Este resultado también sugiere que la adición de la montmorillonita provee protección frente a la degradación fotoquímica causada por la radiación UV, lo cual podría ser interesante desde el punto de vista de aplicaciones de envasado de alimentos. Por otra parte, se puede observar tanto en la Fig. 22 como en la Tabla 6 que la transmisión de luz de todos los nanocomposites PLA-C30 es muy buena, superando el $85 \%$ en todos los casos. Sin embargo, la adición de la montmorillonita causó un ligero descenso en la transmisión de luz del PLA, debido a que las partículas de arcilla (las de mayor tamaño) dispersas en la matriz polimérica dispersan y reflejan la luz, especialmente la de menor longitud de onda. Resultados similares fueron encontrados por Cele et al. [30] en nanocomposites de PLA con 3, 5 y 7\% de C30. Llama también la atención la mayor transmisión de luz mostrada por el PLAR-C30. Esta mejora de la transmisión podría ser debida a la mejor dispersión de la arcilla, que fue observada mediante TEM y XRD. 
Capítulo 4. Efecto del reciclado en estructura y propiedades

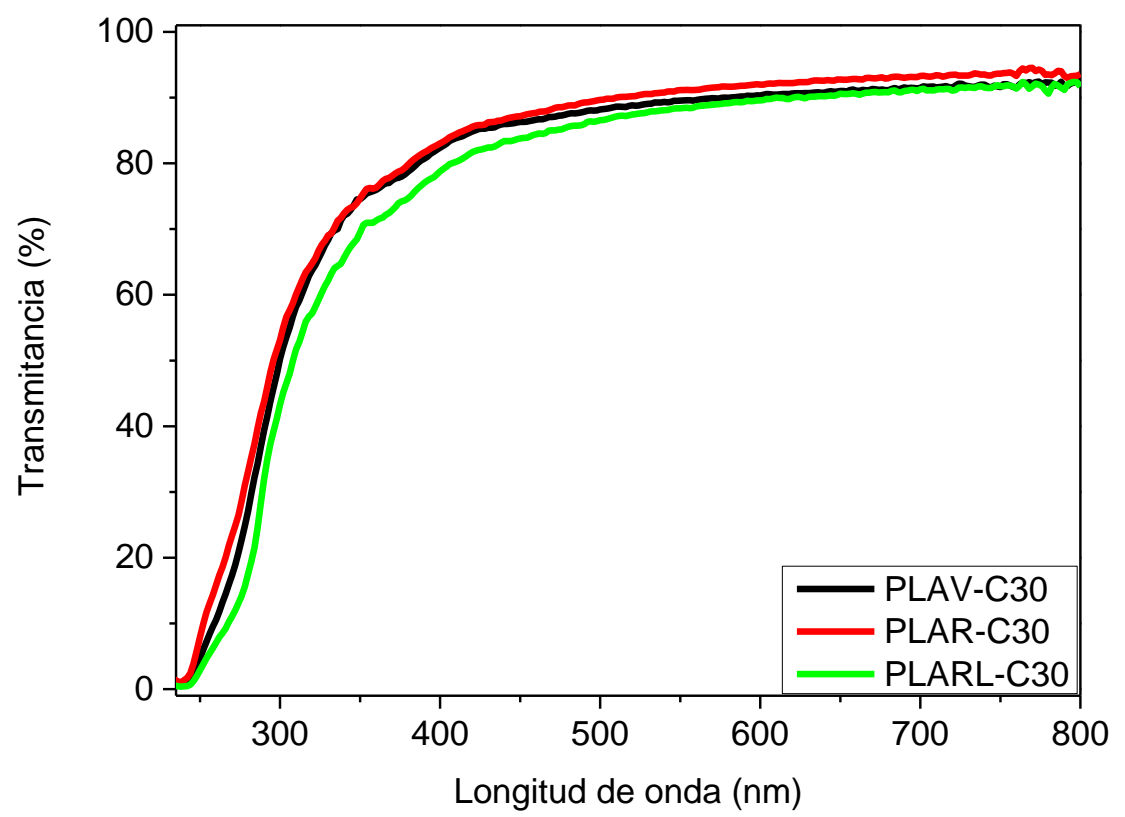

Fig. 22. Espectros UV-Vis del nanocomposite PLA-C30 sometido a diferentes procesos de reciclado mecánico.

Finalmente, en la Fig. 23 se presentan los espectros UV-Vis de los nanocomposites PLA-Ha. En este caso si se puede observar la presencia de la banda de absorción alrededor de $275 \mathrm{~nm}$ en el material reciclado, que es atribuida a la degradación del polímero. En cuanto al comportamiento en la región visible del espectro, ambos materiales siguen la misma tendencia mostrada por el PLA puro y por los nanocomposites PLA-C30, con altos valores de transmisión de luz tanto en PLAV-Ha como en el PLARHa.

En resumen, los resultados de la espectroscopía UV-Vis muestran que la generación de compuestos con grupos terminales carboxilo, debido a la degradación del PLA durante el reciclado, tiene como consecuencia la aparición de una banda de absorción alrededor de $275 \mathrm{~nm}$. Sin embargo, a pesar de la degradación del PLA, la transmisión de luz en todos los casos se acerca al 90\%, sugiriendo que, al menos desde un punto de vista de propiedades ópticas, el PLA y sus nanocomposites reciclados podrían ser utilizados nuevamente en aplicaciones de envasado. 


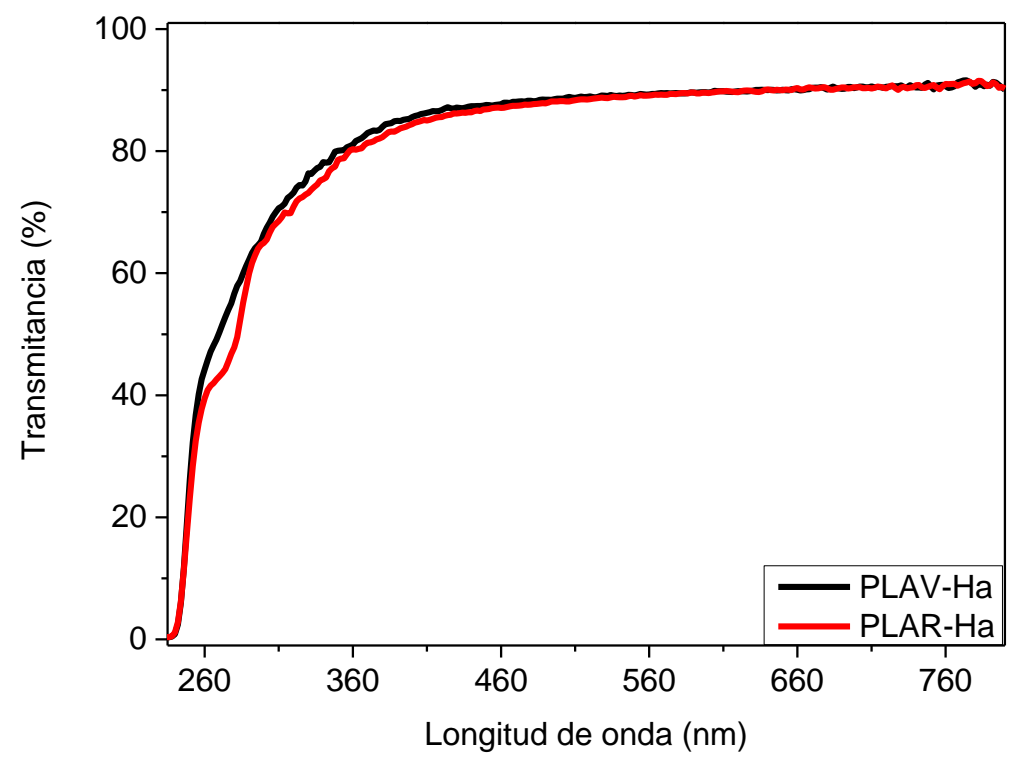

Fig. 23. Espectros UV-Vis del nanocomposite PLA-Ha sometido a diferentes procesos de reciclado mecánico.

\subsection{Microdureza de los materiales reciclados}

Las propiedades mecánicas de los materiales también son muy importantes en las aplicaciones de envasado. Por ello, se ha determinado el efecto de los diferentes procesos de reciclado en la dureza de las diferentes muestras. Cabe destacar que algunos autores, como Pillin et al. [92], sugieren que las medidas de dureza son más sensibles a los cambios en el peso molecular que otras técnicas de caracterización mecánica. En la Fig. 24 se encuentran resumidos los resultados obtenidos.

En primer lugar, es necesario aclarar que los diferentes procesos de reciclado mecánico podrían tener dos efectos contrapuestos en la dureza de los materiales estudiados. Por una parte, el descenso del peso molecular del PLA durante los diferentes procesos de reciclado promueve la reducción de la dureza del polímero, lo que puede ser explicado considerando la influencia del peso molecular en la resistencia y rigidez de los polímeros, así como las relaciones entre la dureza y otras propiedades mecánicas [70,83]. Por otra parte, la generación de nuevos grupos terminales carboxilo, que ya ha sido discutida en los ensayos de espectroscopía UV-Vis, aumenta la interacción entre las cadenas de PLA, lo que causa el endurecimiento del polímero.

En la Fig. 24 se puede observar que el PLAR, PLARL y PLARLH presentan menores valores de dureza que el PLAV. Sin embargo, las diferencias son relativamente pequeñas, del orden del 10\% en el caso más extremo (PLARL). Esta leve disminución de la dureza se encuentra en la misma línea de los resultados obtenidos por otros autores. Por ejemplo, Pillin et al. reporta [92] una disminución de la dureza, medida por nanoindentación, del $17 \%$ en un PLA sometido a 7 ciclos de reprocesado mecánico. Así mismo, esta moderada disminución de la dureza sugiere la ocurrencia de los dos efectos 
mencionados anteriormente, y la prevalencia del efecto negativo de la degradación. Además, es importante destacar que el PLARLH presenta valores de dureza superiores a los del PLAR y PLARL, a pesar de estar sometido a una etapa adicional de envejecimiento. Este comportamiento es difícil de explicar, sobre todo si se toma en cuenta que los valores de viscosidad intrínseca del PLARLH eran bastante próximos a los del PLARL. No obstante, hay que considerar que el PLARLH fue sometido a una etapa adicional de envejecimiento hidrotérmico, el cual podría haber generado un mayor número de grupos reactivos, incrementando las interacciones de cadena, aumentando así la dureza del polímero.

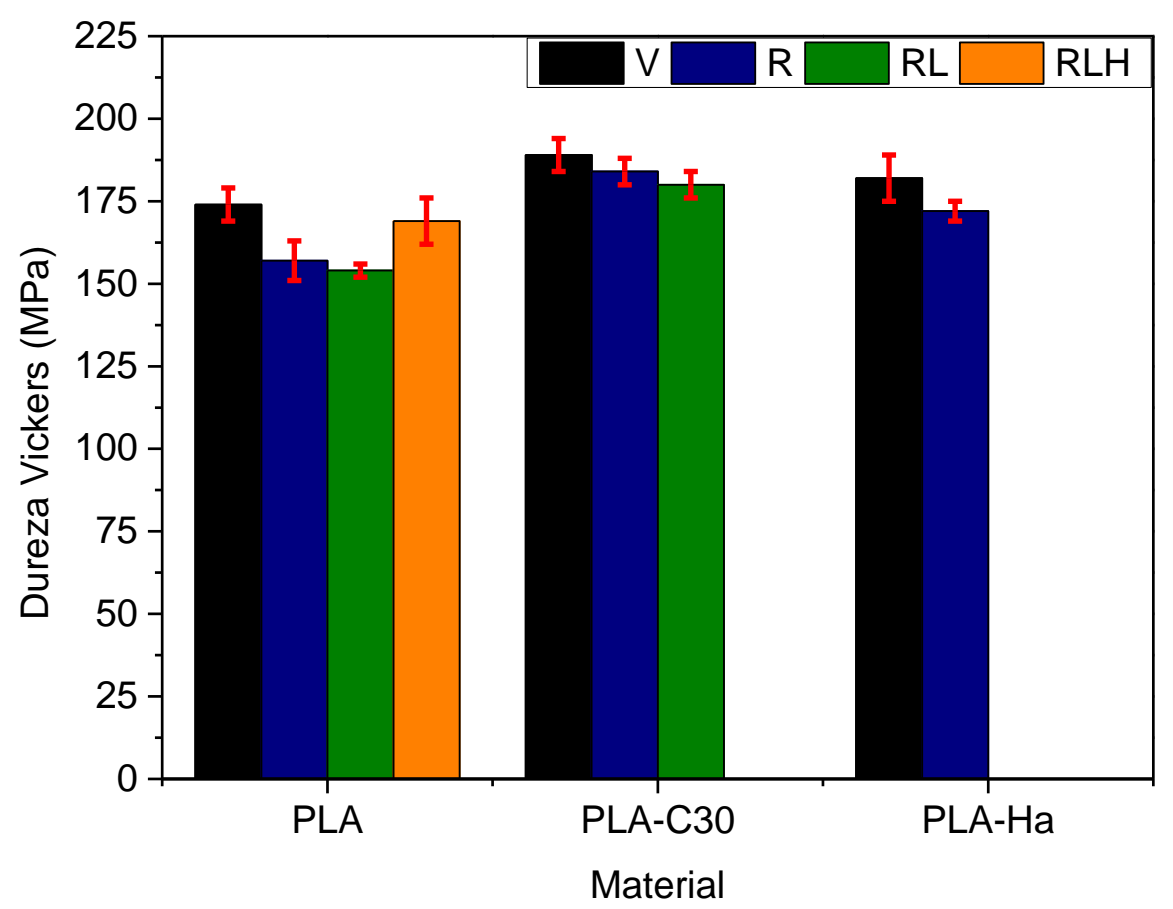

Fig. 24. Valores de microdureza de los diferentes materiales.

En cuanto al efecto de las arcillas, en la Fig. 24 se pueden apreciar algunos resultados interesantes. En primer lugar, ambos nanocomposites vírgenes presentan una mayor dureza que el PLAV (aproximadamente 10\% mayor), indicando que la adición de $2 \%$ de arcilla (C30 o haloisita) tiene un efecto de refuerzo sobre el PLA. Este efecto de refuerzo de pequeñas cantidades de arcilla en la matriz de PLA ya ha sido previamente reportado por autores como Lai et al. [63], quienes obtuvieron un incremento del 5\% en el módulo elástico al añadir 3\% de C30 al PLA, y Zaidi et al. [116], quienes reportan aumentos de entre el 5 y el 19\% al añadir entre 1 y 5\% de C30. En segundo lugar, también se puede observar que, cualitativamente hablando, el efecto del reciclado mecánico en la dureza de los nanocomposites es similar al observado en el PLA puro. Sin embargo, existen diferencias a nivel cuantitativo, ya que la disminución de la dureza es menor en los nanocomposites que en el PLA sin reforzar. Este comportamiento no concuerda con lo observado en las medidas de viscosidad intrínseca, donde se halló que la degradación durante el reciclado era más importante en los nanocomposites que en el polímero puro. 
Este comportamiento podría ser explicado si se considera el efecto de refuerzo que puede tener la mejora en la dispersión de las partículas de arcilla durante el reciclado mecánico, observada mediante XRD y microscopía electrónica, lo que aumentaría la dureza de los nanocomposites reciclados, contrarrestando así el efecto negativo de la degradación.

Resumiendo, el efecto de los diferentes procesos de reciclado mecánico tanto en el PLA como en sus nanocomposites parece ser moderado. Por lo tanto, los resultados parecen indicar que las propiedades mecánicas de los materiales reciclados no deberían ser un factor limitante a la hora de considerar estos materiales para aplicaciones de envasado.

\subsection{Propiedades de transporte de los materiales reciclados}

La permeabilidad frente a diferentes gases es una propiedad crucial en los materiales empleados en el envasado de alimentos frescos, ya que influye de forma importante en la vida útil de los productos envasados. Por lo tanto, es importante evaluar el efecto de los diferentes procesos de reciclado en la permeabilidad de diferentes gases a través de los diferentes materiales. Los resultados obtenidos se encuentran recogidos en la Fig. 25.

En primer lugar, se puede observar que le permeabilidad del PLA frente a los diferentes gases estudiados parece aumentar ligeramente con los diferentes procesos de reciclado. Un resultado similar fue encontrado por Żenkiewicz et al. [117] al estudiar el reprocesado mecánico del PLA. Estos autores reportaron un incremento del $20 \%$ en la permeabilidad frente al oxígeno en el PLA reprocesado 10 veces. Este comportamiento podría ser consecuencia de la degradación del PLA durante el reciclado. La reducción del peso molecular del PLA ocasiona un aumento del volumen libre dentro del polímero, y, de acuerdo a la ecuación de Doolitle, la difusión del gas a través de polímeros vítreos es más fácil cuanto mayor sea el volumen libre dentro del material [36]. 
Capítulo 4. Efecto del reciclado en estructura y propiedades

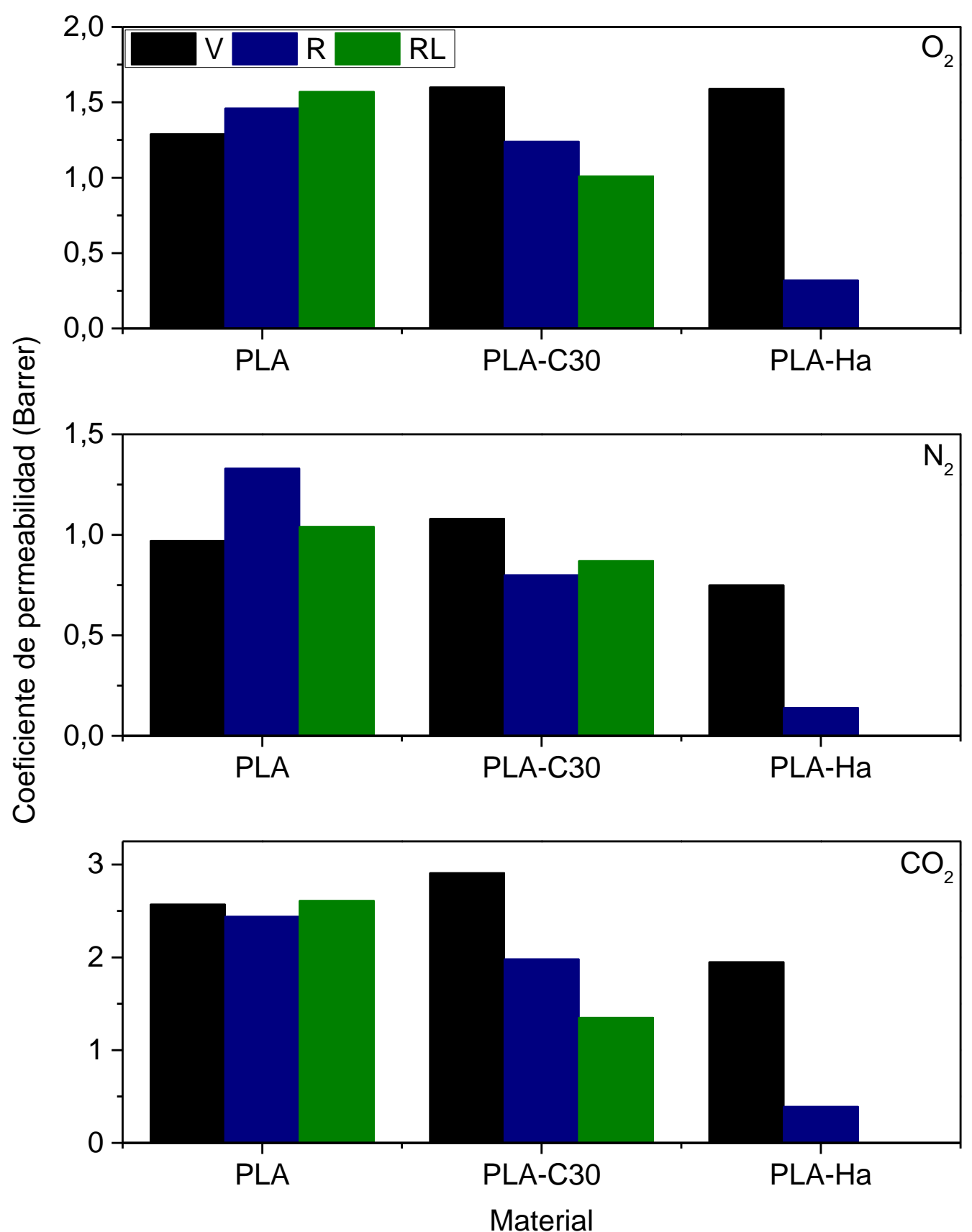

Fig. 25. Coeficiente de permeabilidad de los diferentes materiales frente al $\mathrm{O}_{2}, \mathrm{~N}_{2} \mathrm{y}$ $\mathrm{CO}_{2}$.

En segundo lugar, la Fig. 25 muestra que la adición tanto de la C30 como de la haloisita produce un ligero incremento en la permeabilidad del PLA. Este resultado no concuerda con lo reportado en la literatura, ya que la adición de las partículas de arcilla debería incrementar la tortuosidad de los caminos seguidos por la moléculas de gas al difundir a través del polímero, lo que llevaría a menores valores de permeabilidad [34,82]. De hecho, varios trabajos, como aquellos realizados por Duan et al. [46], Molinaro et al [76] y Rhim et al. [100], reportan una mejora de las propiedades de barrera del PLA frente 
a diferentes gases como vapor de agua y oxígeno al añadir pequeñas cantidades de arcillas modificadas orgánicamente. Sin embargo, también hay que considerar que los nanocomposites vírgenes presentan un menor peso molecular que el PLAV, tal y como se observó en las medidas de viscosidad intrínseca, lo que implica un mayor volumen libre y, por lo tanto, una mayor permeabilidad. Los resultados obtenidos en este estudio sugieren que el efecto de la viscosidad intrínseca en las propiedades de barrera predomina sobre el incremento de la tortuosidad, al menos en las condiciones empleadas en este trabajo.

En tercer lugar, se puede observar en la Fig. 25 que los nanocomposites reciclados presentan menores valores del coeficiente de permeabilidad que los nanocomposites vírgenes, a pesar de que las medidas de viscosidad evidencian la degradación del PLA. Para entender este comportamiento es necesario considerar la dispersión de las arcillas en la matriz polimérica. Las fotografías TEM y los ensayos XRD muestran que el reciclado mecánico mejora la dispersión de las arcillas, promoviendo incluso la exfoliación de la montmorillonita. Por lo tanto, los resultados mostrados indican que la mejora en la dispersión de los nanocomposites prevalece sobre el efecto negativo de la degradación en la permeabilidad de los nanocomposites reciclados. De hecho, los filmes con menor permeabilidad son aquellos que fueron sometidos a procesos más enérgicos (como la etapa de lavado), que a pesar de degradar de forma significativa el PLA, condujeron a una mejor dispersión de la arcilla.

En resumen, los resultados obtenidos indican que el reciclado mecánico tiene un efecto limitado en las propiedades de barrera frente a gases del PLA y sus nanocomposites. En el caso del PLA puro, los materiales reciclados presentaron un ligero aumento en la permeabilidad frente a diferentes gases, mientras que en los nanocomposites los materiales reciclados presentaron mejores propiedades de barrera. Estos resultados parecen señalar que el PLA reciclado, y sus nanocomposites, podrían ser utilizados en aplicaciones de envasado, al menos desde un punto de vista de sus propiedades de transporte. 


\section{EFECTO DEL RECICLADO EN LA DEGRADACIÓN HIDROLÍTICA DEL PLA Y SUS NANOCOMPOSITES}

Como se ha mencionado con anterioridad, una de las principales aplicaciones del PLA es el envasado de alimentos. En este tipo de aplicaciones, cabe esperar que el polímero se encuentre en contacto con líquidos, o alimentos húmedos, que promuevan la degradación hidrolítica del PLA. Por ello, es importante evaluar el efecto de los diferentes procesos de reciclado en la degradación hidrolítica del PLA y sus nanocomposites.

\subsection{Cinética de la absorción de agua de los materiales reciclados}

La absorción de agua podría catalogarse como la etapa inicial del proceso de degradación hidrolítica, por lo que es importante evaluar si los diferentes procesos de reciclado mecánico afectan a la cinética de absorción, ya que esto podría influir en la degradación hidrolítica del PLA.

La Fig. 26 muestra las curvas de absorción de agua del PLA virgen y sometido a diferentes procesos de reciclado mecánico. Se puede observar que en todos los casos la cantidad de agua absorbida aumenta continuamente, sin estabilizarse en ningún momento. Este comportamiento ha sido previamente reportado en la literatura. Por ejemplo, Davis et al. [42] encontraron un comportamiento similar al analizar, mediante espectroscopía infrarroja y gravimetría, la difusión de agua a través de filmes finos de PLA. Este fenómeno podría ser atribuido a la naturaleza de no-equilibrio del PLA vítreo, que causa una cinética de absorción en dos etapas: la primera, a tiempos cortos, en la que la difusión es impulsada por el gradiente de concentración; y la segunda, a tiempos largos, donde el fenómeno predominante es la relajación e hinchamiento del polímero [19]. Otros autores, como Deroiné et al. [43], consideran también que la degradación del PLA causa un incremento de la cantidad de agua absorbida a tiempos largos, ya que genera agujeros y compuestos hidrofílicos, los cuales facilitarían la penetración del agua en el polímero. 


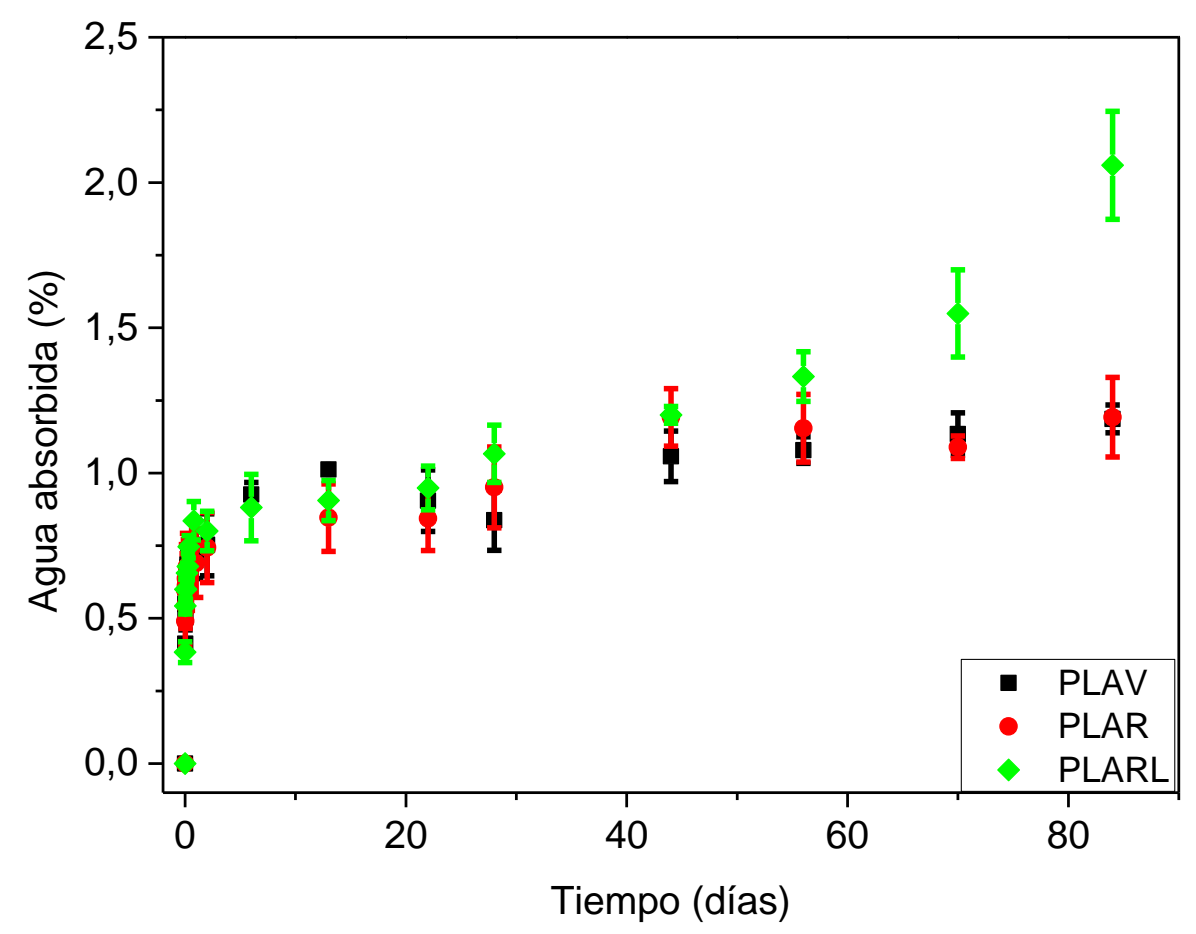

Fig. 26. Curvas de absorción de agua, a $37^{\circ} \mathrm{C}$, del PLA sometido a diferentes procesos de reciclado mecánico.

A pesar de que los tres materiales estudiados presentan una tendencia similar, existen diferencias significativas entre el material sometido a una etapa de lavado, y aquellos que no fueron sometidos a dicho proceso. En la Fig. 26 se puede apreciar que, mientras que las curvas de absorción del PLAV y el PLAR prácticamente se superponen durante todo el tiempo de inmersión, el PLARL comienza a mostrar un mayor incremento en la cantidad de agua absorbida después de 28 días de inmersión, diferencia que se hace más evidente después de 56 días de experimento. Este comportamiento podría estar relacionado con la mayor degradación del PLARL, tal y como se observó en las medidas de viscosidad intrínseca. Y es que las cadenas más cortas presentes en este material poseen una mayor movilidad, lo que facilitaría los procesos de relajación e hinchamiento, aumentando así la cantidad de agua absorbida por el material a tiempos largos de inmersión.

El hecho de que la cantidad de agua absorbida aumente constantemente indica que el conjunto del proceso de absorción no puede ser descrito utilizando un modelo Fickiano, ya que este asume que el transporte está controlado únicamente por la difusión y que finalmente se alcanza una situación de equilibrio. En nuestro caso esa situación de equilibrio no se alcanza debido a que los modelos Fickianos dejan de lado otros procesos, muy importantes en el caso del PLA, como la relajación molecular y la degradación hidrolítica. 
Sin embargo, sería muy importante determinar si a tiempos cortos, cuando los procesos de relajación y degradación deberían ser aún poco importantes, la difusión se encuentra regida por el gradiente de concentración. Si eso fuera así, sería posible describir esta parte del proceso de absorción de agua utilizando un modelo Fickiano.

Este enfoque haría posible el estudio del efecto de los diferentes procesos de reciclado mecánico en la cinética de absorción de agua del PLA, ya que permitiría estimar tanto el coeficiente de difusión como la cantidad de agua en el equilibrio de los diferentes materiales.

Para estimar los parámetros de absorción de los diferentes materiales se empleó una solución numérica de la segunda ley de Fick, en la que se considera que la absorción ocurre en un plano (idealización de las láminas estudiadas en este trabajo), bajo flujo unidireccional y con un coeficiente de difusión constante [40]:

$$
\frac{M_{t}}{M_{\infty}}=1-\frac{8}{\pi^{2}} \sum_{n=0}^{\infty} \frac{1}{(2 n+1)^{2}} \exp \left(\frac{-(2 n+1)^{2} \pi^{2} D t}{h^{2}}\right)
$$

donde $M_{t}$ es el agua absorbida en un tiempo $t, M_{\infty}$ es la cantidad de agua en el equilibrio, $n$ es el número de términos de la solución empleados en el cálculo, $D$ es el coeficiente de difusión y $h$ es el espesor de la muestra. Es importante destacar que, en una primera etapa de los cálculos se determinó que el valor óptimo de $n$ era 10, ya que no se obtenían mejores ajustes al utilizar valores más altos.

Los valores del coeficiente de difusión y del contenido de agua en el equilibrio fueron obtenidos ajustando los datos experimentales, a tiempos cortos de inmersión, utilizando la ecuación 7. Sin embargo, es necesario considerar las dimensiones de las probetas a la hora de reportar los valores del coeficiente de difusión, por lo que los valores obtenidos fueron corregidos utilizando la ecuación 8 [52]:

$$
D c=D \times\left(1+\frac{h}{x}+\frac{h}{y}\right)
$$

donde $D c$ es el coeficiente de difusión corregido y $h, x$ e $y$ son el espesor, longitud y ancho de las probetas.

En la Fig. 27 se presenta el resultado de ajustar los datos experimentales del PLAV, PLAR y PLARL a $37{ }^{\circ} \mathrm{C}$ empleando la ecuación 7. Se puede observar que los datos correspondientes a tiempos cortos de inmersión pueden ser adecuadamente ajustados utilizando la ecuación 7 , resultado que se confirma al observar los bajos valores de $\chi^{2}$ observados en la Tabla 7. Este comportamiento indica que, a tiempos cortos de 
inmersión, el fenómeno de absorción de agua en el PLA virgen y reciclado puede ser descrito utilizando un modelo Fickiano.
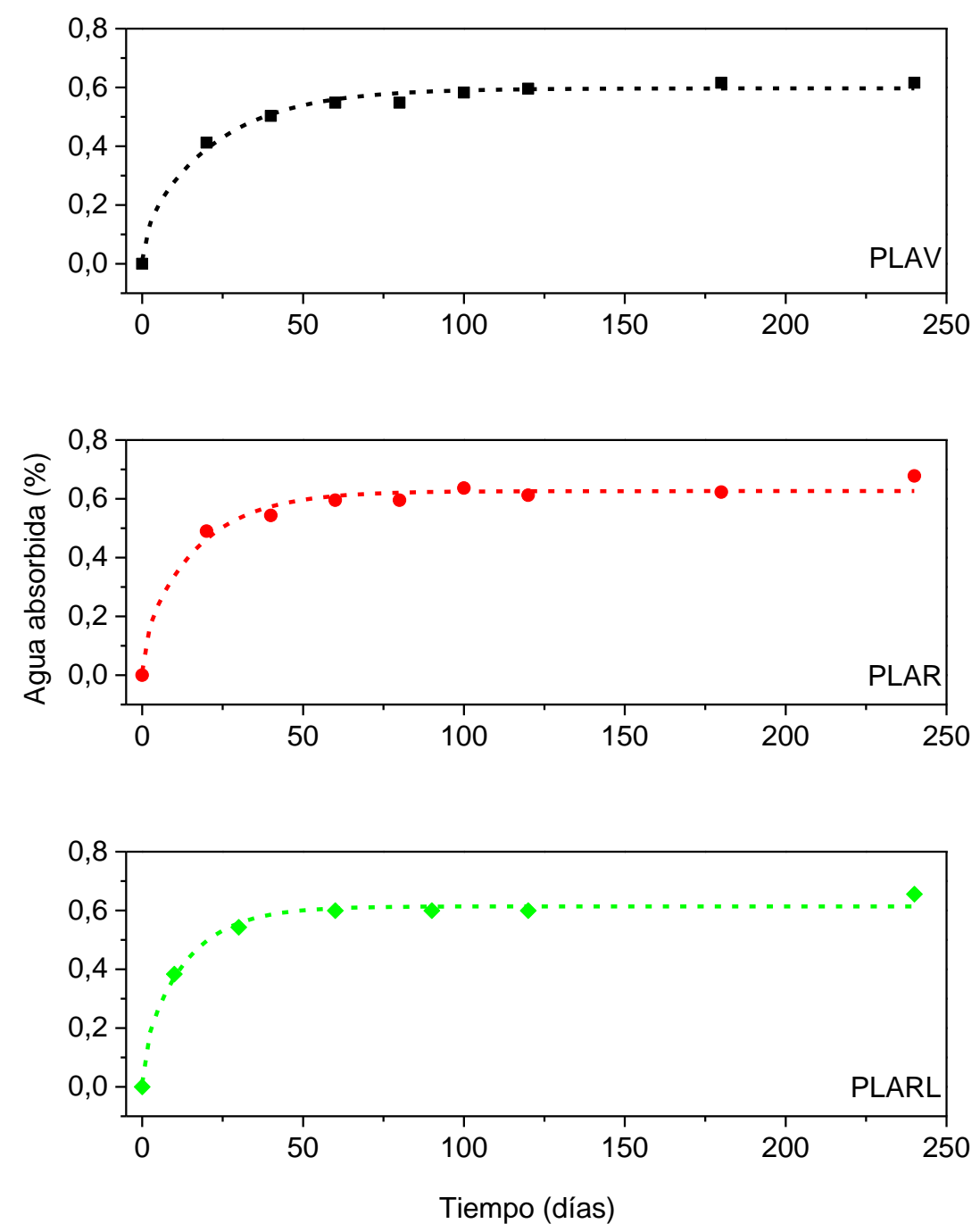

Fig. 27. Ajuste de los datos de absorción de agua, a $37^{\circ} \mathrm{C}$, del PLA sometido a diferentes procesos de reciclado mecánico, utilizando la ecuación 7.

Los valores del coeficiente de difusión, junto con los del contenido de agua en el equilibrio se presentan en la Tabla 7. Por una parte, se observa que los valores de $M_{\infty}$ son muy parecidos en el PLAV, PLAR y PLARL, sugiriendo que el reciclado mecánico no afecta de forma significativa la cantidad de agua que absorbe el PLA a tiempos cortos. Por otra parte, el PLAR y, especialmente, el PLARL presentan valores más grandes del coeficiente de difusión que el PLAV. Este comportamiento podría explicarse tomando en cuenta dos factores: primero, la degradación del PLA durante el reciclado mecánico genera compuestos hidrofílicos que facilitarían la difusión del agua; el segundo factor 
está relacionado con un aumento del volumen libre dentro del polímero, debido al menor peso molecular, que ocasionaría un incremento del coeficiente de difusión.

Tabla 7. Parámetros de absorción de agua, a $37^{\circ} \mathrm{C}$, de los diferentes materiales vírgenes y reciclados.

\begin{tabular}{cccc}
\hline Material & $\mathbf{D}_{\mathbf{c}} \mathbf{x} \mathbf{1 0}^{\mathbf{8}}\left(\mathbf{c m}^{\mathbf{2}} / \mathbf{s}\right)$ & $\mathbf{M}_{\infty}(\boldsymbol{\%})$ & $\boldsymbol{\chi}^{\mathbf{2}}$ \\
\hline PLAV & $3,7 \pm 1,6$ & $0,58 \pm 0,01$ & 0,0002 \\
PLAR & $4,2 \pm 1,8$ & $0,61 \pm 0,01$ & 0,0004 \\
PLARL & $7,3 \pm 2,0$ & $0,60 \pm 0,01$ & 0,00007 \\
\hline PLAV-C30 & $4,2 \pm 1,2$ & $0,61 \pm 0,01$ & 0,0001 \\
PLAR-C30 & $3,8 \pm 1,5$ & $0,62 \pm 0,01$ & 0,00003 \\
PLARL-C30 & $5,7 \pm 1,6$ & $0,65 \pm 0,01$ & 0,0002 \\
\hline
\end{tabular}

En cuanto a la absorción de agua de los nanocomposites de PLA, en la Fig. 28 se muestran las curvas de absorción del nanocomposite PLA-C30 sometido a diferentes procesos de reciclado. En primer lugar, se puede observar que el comportamiento de los nanocomposites es similar al del PLA puro, donde los materiales virgen y reciclado sin lavar presentan una curva similar, mientras que el material lavado muestra una mayor absorción a tiempos largos de inmersión. En segundo lugar, si se comparan la Fig. 28 y la Fig. 26, se aprecia claramente que la cantidad de agua absorbida por los nanocomposites es superior a la del PLA puro. Este comportamiento podría ser atribuido al carácter hidrofílico de las partículas de arcilla, lo que promovería una mayor absorción de agua. Sin embargo, la cantidad de arcilla en el material es reducida, por lo que también podría influir la degradación del PLA observada en los nanocomposites con C30.

En lo que refiere a los parámetros de absorción de agua de los nanocomposites, por una parte, en la Tabla 7 se puede observar que los nanocomposites presentan coeficientes de difusión similares a los del PLA puro. Este resultado es llamativo, ya que la presencia de las láminas de arcilla dispersas en el PLA aumenta la tortuosidad del material, y por tanto debería disminuir el coeficiente de difusión [34]. Sin embargo, no se puede obviar la mayor degradación observada en los nanocomposites PLA-C30, que facilitaría también la difusión de las moléculas de agua a través de la matriz polimérica.

Por otra parte, la Tabla 7 muestra que el efecto del reciclado en el coeficiente de difusión de los nanocomposites PLA-C30 es diferente al observado en PLA puro, ya que el PLAR-C30 muestra un menor coeficiente de difusión que el PLAV-C30, mientras que el PLARL-C30 presenta un coeficiente de difusión mayor. Para explicar este comportamiento es necesario considerar de nuevo los cambios estructurales y morfológicos ocurridos durante el reciclado mecánico de los nanocomposites. En un lado está la degradación del PLA, responsable del descenso de la viscosidad intrínseca, que ocasiona un aumento del coeficiente de difusión (tal como se ha visto en el PLA puro). En otro lado se encuentra la mejora en la dispersión de las arcillas, observada mediante XRD y TEM, que puede ocasionar una disminución del coeficiente de difusión. En el 
caso del PLAR-C30, la degradación era relativamente pequeña, por lo que prevalece el efecto de la mejora en la dispersión de la arcilla. En el caso del PLARL-C30, la degradación era más grande, por lo que, a pesar de la mejora en la dispersión de la arcilla, el coeficiente de difusión aumenta [16].

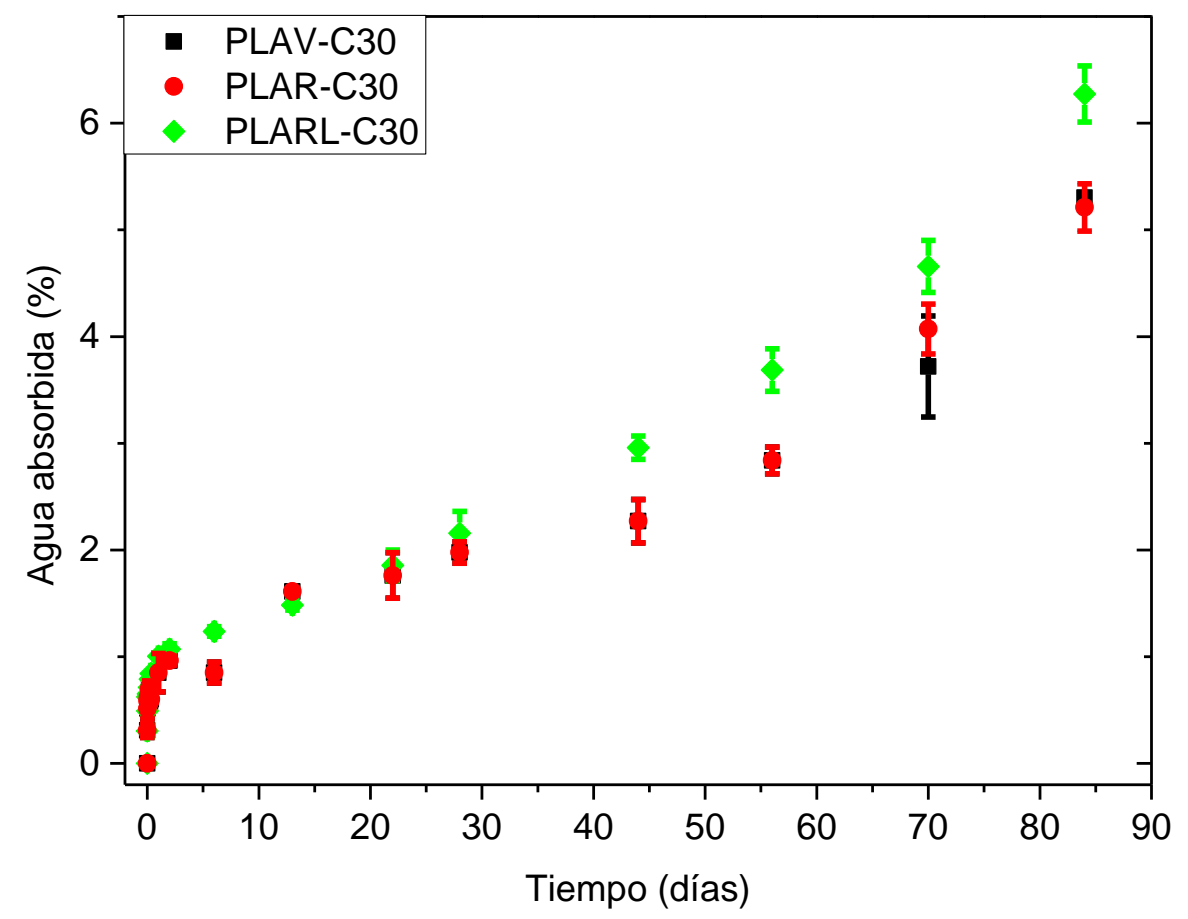

Fig. 28. Curvas de absorción de agua, a $37^{\circ} \mathrm{C}$, de los nanocomposites PLA-C30 sometidos a diferentes procesos de reciclado.

Además de los ensayos a $37^{\circ} \mathrm{C}$, también se estudió la cinética de absorción de algunos materiales a $58^{\circ} \mathrm{C}$, una temperatura próxima a la transición vítrea del PLA y que además a menudo se ha considerado representativa de los procesos industriales de compostaje. Si se comparan la Fig. 27 y la Fig. 29, y la Tabla 7 y la Tabla 8, se puede observar que a $58{ }^{\circ} \mathrm{C}$ la absorción de agua es mayor y más rápida que a $37{ }^{\circ} \mathrm{C}$. Estos resultados coinciden con los reportados en la literatura por Deroiné et al. [43], y podrían ser explicados tomando en cuenta el efecto activador de la temperatura en la habilidad del agua para modificar la red polimérica, y así "abrir" sitios polares para la asociación, multiplicando los puentes de hidrógeno entre las moléculas de agua y el PLA.

Esta activación térmica también se traduce en un incremento en el coeficiente de difusión, que pasa a ser un orden de magnitud mayor en ambos materiales, obteniendo valores de $(18 \pm 8) \mathrm{cm}^{2} / \mathrm{s}$ en el PLAV y $(10 \pm 2) \mathrm{cm}^{2} / \mathrm{s}$ en el PLAR. Cabe destacar que el material reciclado presenta valores menores de $M_{\infty} \mathrm{y}$ del coeficiente de difusión que el material virgen. Este llamativo comportamiento podría ser explicado teniendo en cuenta la cristalización del PLA a $58^{\circ} \mathrm{C}$, fenómeno que se analizará en detalle más adelante. Las 
cadenas más cortas presentes en el PLAR cristalizarían con mayor facilidad que las del PLAV, actuando como una barrera frente a la absorción de agua.

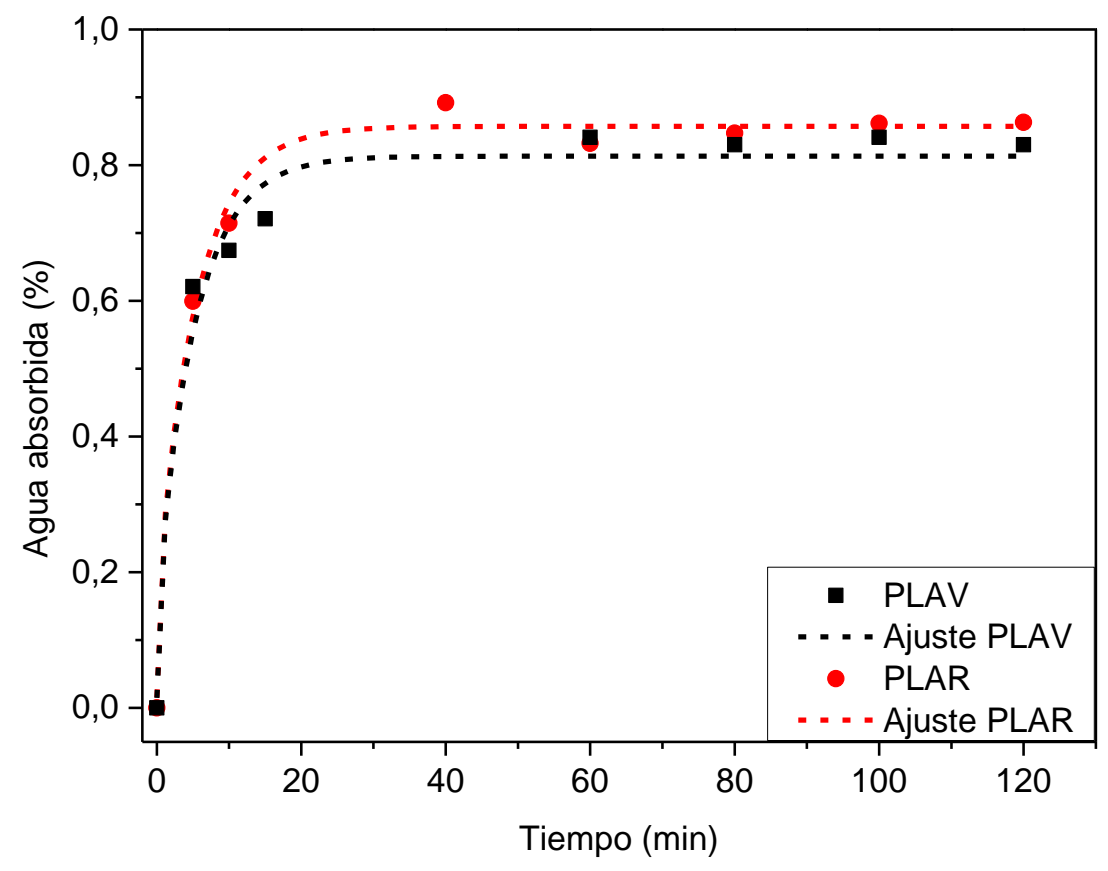

Fig. 29. Ajuste de los datos de absorción de agua, a $58^{\circ} \mathrm{C}$, del PLAV y PLAR

En el caso de los nanocomposites PLA-C30, se puede observar en la Tabla 8 que estos absorben una mayor cantidad de agua que el PLA puro. Como se ha explicado anteriormente, esta diferencia se debe al carácter hidrofílico de las arcillas, que promueven la absorción de agua en el PLA. En cuanto a los valores del coeficiente de difusión, se puede apreciar que los nanocomposites no muestran un aumento del coeficiente de difusión y que los valores obtenidos son menores que los del PLA puro. Este resultado podría explicarse por la capacidad de las partículas de arcilla para limitar la difusión del agua dentro del PLA, y por el efecto que pudiera tener la presencia de la C30 en la formación de estructuras cristalinas más ordenadas en el PLA, lo que contribuiría a menores valores del coeficiente de difusión.

Tabla 8. Parámetros de absorción de agua, a $58^{\circ} \mathrm{C}$, de los diferentes materiales vírgenes y reciclados.

\begin{tabular}{cccc}
\hline Material & $\mathbf{D}_{\mathbf{c}} \mathbf{x} \mathbf{1 0}^{\mathbf{8}}\left(\mathbf{c m}^{\mathbf{2}} / \mathbf{s}\right)$ & $\mathbf{M}_{\infty}(\boldsymbol{\%})$ & $\boldsymbol{\chi}^{\mathbf{2}}$ \\
\hline PLAV & $18,0 \pm 8,0$ & 0,86 & 0,002 \\
PLAR & $9,7 \pm 1,8$ & 0,82 & 0,002 \\
\hline PLAV-C30 & $5,8 \pm 2,6$ & 1,12 & 0,006 \\
PLAR-C30 & $7,1 \pm 3,4$ & 1,03 & 0,001 \\
\hline
\end{tabular}


En resumen, los resultados obtenidos a $37^{\circ} \mathrm{C}$ indican que los diferentes procesos de reciclado ocasionan un incremento del coeficiente de difusión del PLA, debido a la degradación del polímero durante el reciclado. Sin embargo, el efecto es pequeño. En el caso de los nanocomposites el comportamiento es más complejo, por una parte, la mejor dispersión de la arcilla disminuye el coeficiente de difusión (tal y como se observa en el PLAR-C30), mientras que por otra parte la degradación del PLA aumenta el coeficiente de difusión (como en el caso del PLARL-C30). A $58^{\circ} \mathrm{C}$ se observa que la absorción de agua es mayor y más rápida; además, a esta temperatura parece hacerse más evidente el efecto de las arcillas sobre el coeficiente de difusión del PLA. Sin embargo, hay que recalcar que esta temperatura se encuentra muy próxima a la transición vítrea del polímero, por lo que los resultados obtenidos son difícilmente extrapolables.

En cualquier caso, los cambios en el coeficiente de difusión afectan la absorción de agua de los materiales y, a largo plazo, podrían influir también en la degradación hidrolítica de los mismos. Por ello, a continuación, se estudiarán los productos de la degradación hidrolítica del PLA y, seguidamente, se analizará el efecto del reciclado mecánico en la evolución de la viscosidad intrínseca, estructura y propiedades del PLA y sus nanocomposites durante la inmersión.

\subsection{Estudio de los productos de degradación mediante espectroscopía UV-Vis}

Como se ha comentado anteriormente la absorción de agua puede considerarse como la primera etapa de la degradación hidrolítica del PLA. Tal como se ilustra en la Fig. 30, en medio básico o neutro la degradación hidrolítica se lleva a cabo mediante transesterificación intramolecular, o backbitting. Durante este proceso se pueden generar ácido láctico, ácido lactoil-láctico (dímero lineal del ácido láctico) y lactida (dímero cíclico del ácido láctico), entre otros, que constituyen los principales agentes migrantes del PLA [37,79]. La presencia de estos compuestos es muy relevante desde el punto de vista del envasado de alimentos, ya que se debe asegurar que los materiales utilizados en el envasado no sean perjudiciales para la salud de los consumidores. Esto incluye que la migración de productos desde el envase al alimento esté por debajo de unos límites, que dependen de la toxicidad de cada producto y que se encuentran definidos por la normativa europea [1].

Dada la importancia de la migración de los productos de degradación del PLA, en este trabajo se ha estudiado el efecto del reciclado mecánico dicho proceso, para ello se han realizado espectros UV-Vis del líquido antes y después de la inmersión. En la Fig. 31 se muestran los espectros UV-Vis del líquido de inmersión del PLA virgen y reciclado, a 37 (después de 100 días de inmersión) y $58^{\circ} \mathrm{C}$ (después de 6 días de inmersión). En primer lugar, es importante destacar que, después de la inmersión, en todos los casos se observa la aparición de tres bandas de absorción, ubicadas a 228, 248 y $290 \mathrm{~nm}$ respectivamente. Estas bandas pueden ser relacionadas con los diferentes productos de la degradación del PLA que han migrado al medio de inmersión, ya que pueden asignarse a las transiciones $\mathrm{n} \rightarrow \pi^{*}$ de los grupos $\mathrm{C}=\mathrm{O}$ de los diferentes ácidos, lactida y ésteres formados en la 
hidrólisis. Por ejemplo, la banda de $248 \mathrm{~nm}$ puede asignarse a oligómeros del ácido láctico y la banda de $290 \mathrm{~nm}$ ha sido relacionada por algunos autores con la presencia de grupos - $\mathrm{COOH}$ en los extremos de las cadenas de PLA [50,53,109].

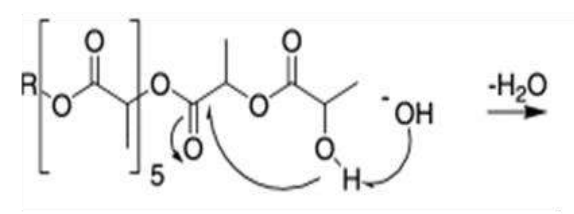

Oligómero de ácido láctico

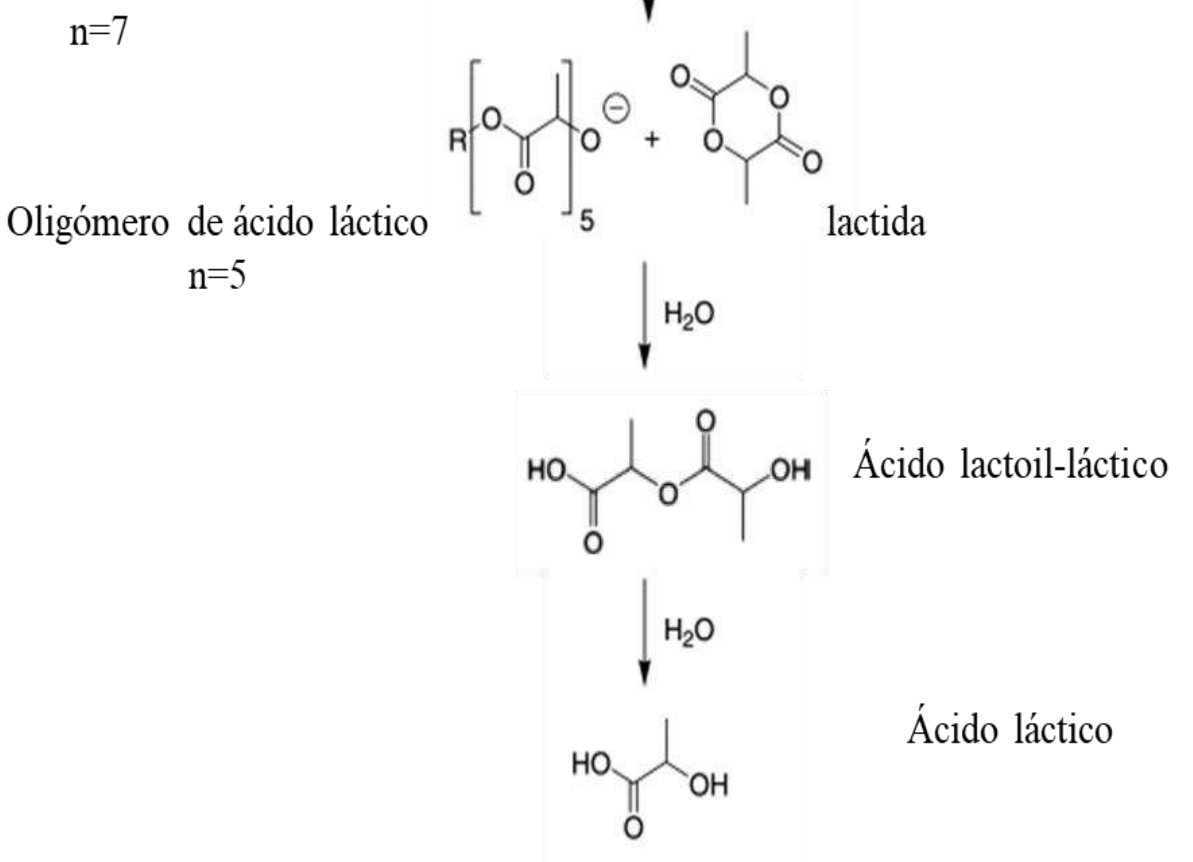

Fig. 30. Mecanismo de degradación hidrolítica del PLLA en medio básico o neutro.

En la Fig. 31 también se evidencia que después de 6 días de inmersión a $58^{\circ} \mathrm{C}$ la presencia de los productos de degradación del PLA es más grande que después de 100 días a $37^{\circ} \mathrm{C}$. Resultados similares han sido reportados por Mutsuga et al. [79], quienes hallaron que los migrantes del PLA aumentaban de forma considerable después de 10 días de inmersión a $60{ }^{\circ} \mathrm{C}$, mientras que a $40{ }^{\circ} \mathrm{C}$ los niveles eran muy bajos, incluso después de 180 días de inmersión. Este resultado indica que la degradación del PLA transcurre a una mayor velocidad a temperaturas más altas, que podría estar relacionada con una mayor velocidad de las reacciones de hidrólisis y, a que a temperaturas próximas a $T_{g}$, la absorción de agua y la difusión de los productos de degradación es mayor. 


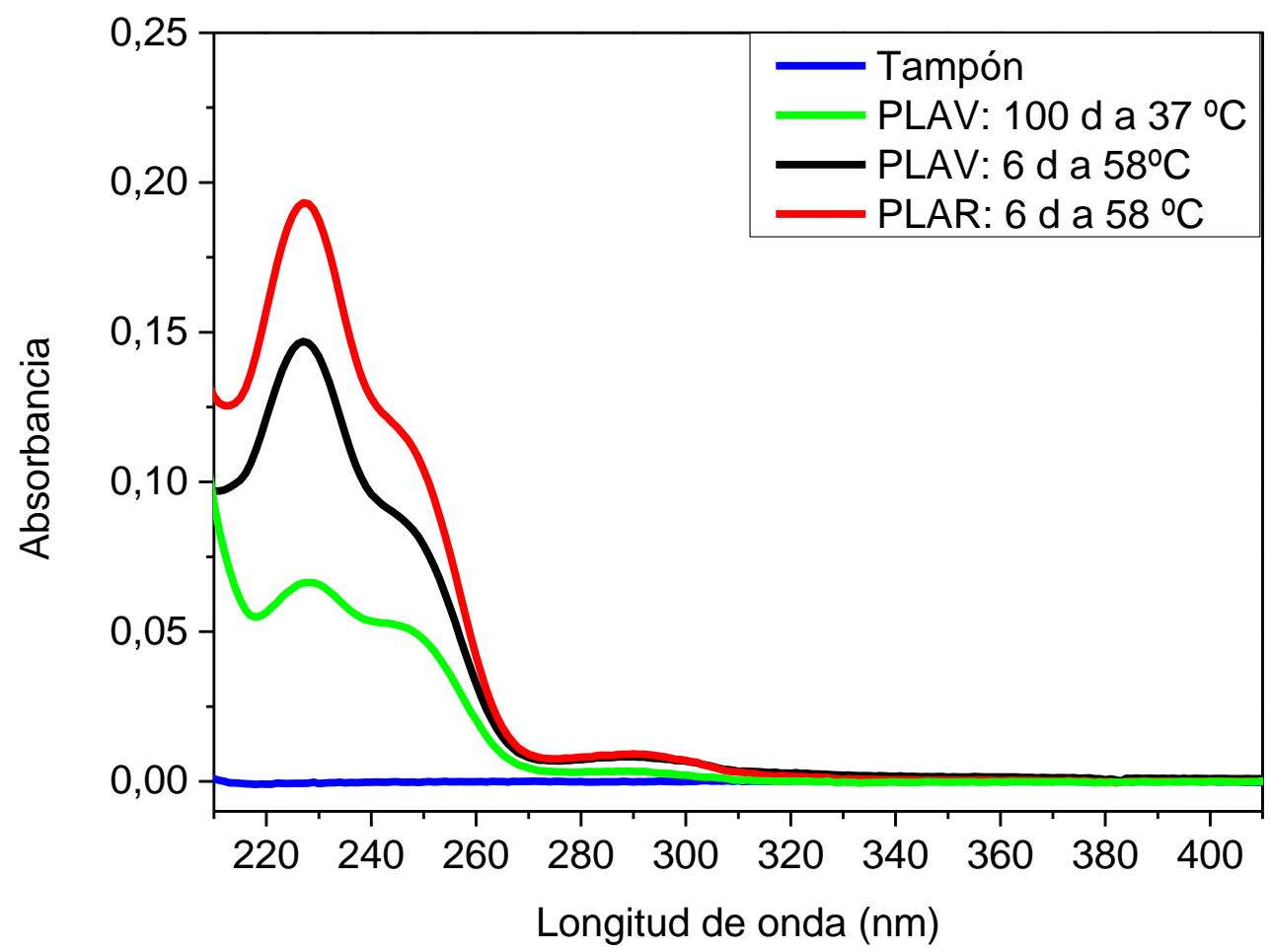

Fig. 31. Espectros UV-Vis del líquido de inmersión, a diferentes temperaturas, del PLA virgen y reciclado.

En cuanto al efecto del reciclado, se puede observar tanto en la Fig. 31 (PLA puro) como en la Fig. 32 (nanocomposite con C30) que los espectros correspondientes al líquido de inmersión de los materiales reciclados presentan una mayor absorbancia después de 6 días de inmersión a $58{ }^{\circ} \mathrm{C}$, lo que sugiere que el reciclado mecánico puede aumentar la degradación del polímero, o la difusión de los productos de la degradación al medio de inmersión. En cualquier caso, las diferencias son bastante pequeñas, por lo que, al menos desde un punto de vista de seguridad alimentaria, no podría descartarse el uso de PLA reciclado en el envasado de alimentos, aunque serían necesarios estudios más detallados para poder asegurar que la seguridad de los consumidores no está comprometida al emplear PLA reciclado. 


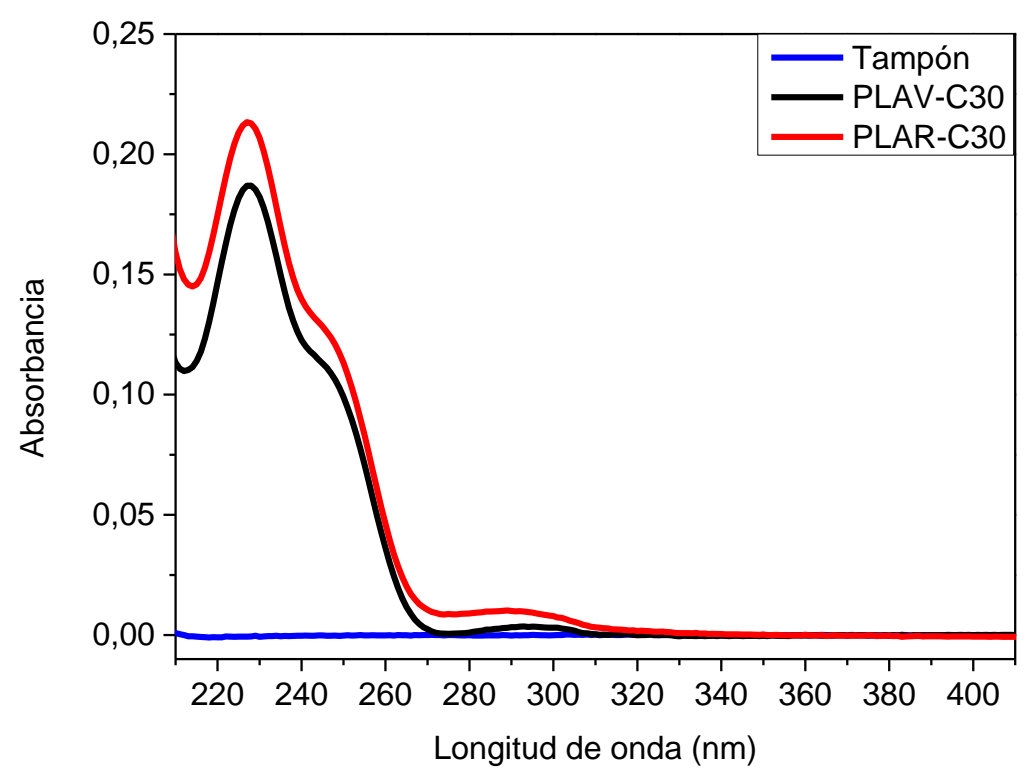

Fig. 32. Espectros UV-Vis del líquido de inmersión, después de 6 días de inmersión a $58^{\circ} \mathrm{C}$ del nanocomposite PLA-C30.

\subsection{Evolución de la viscosidad intrínseca durante la inmersión de los materiales reciclados}

Los migrantes observados en la sección anterior son consecuencia de la degradación hidrolítica del PLA, la cual causa un descenso del peso molecular y puede afectar la estructura y algunas propiedades del polímero. En la Fig. 33 se muestra la evolución de la viscosidad intrínseca del PLA virgen y sometido a diferentes procesos de reciclado, sumergido a $37^{\circ} \mathrm{C}$. Se puede observar que después de 84 días de inmersión, todos los materiales presentan un descenso de la viscosidad intrínseca de alrededor del $30 \%$, y las diferencias observadas durante la inmersión se deben a la menor viscosidad inicial de los materiales reciclados. Estos resultados sugieren que la resistencia frente a la degradación hidrolítica, al menos desde el punto de vista de la viscosidad intrínseca, no se ve afectada por los diferentes procesos de reciclado mecánico. 


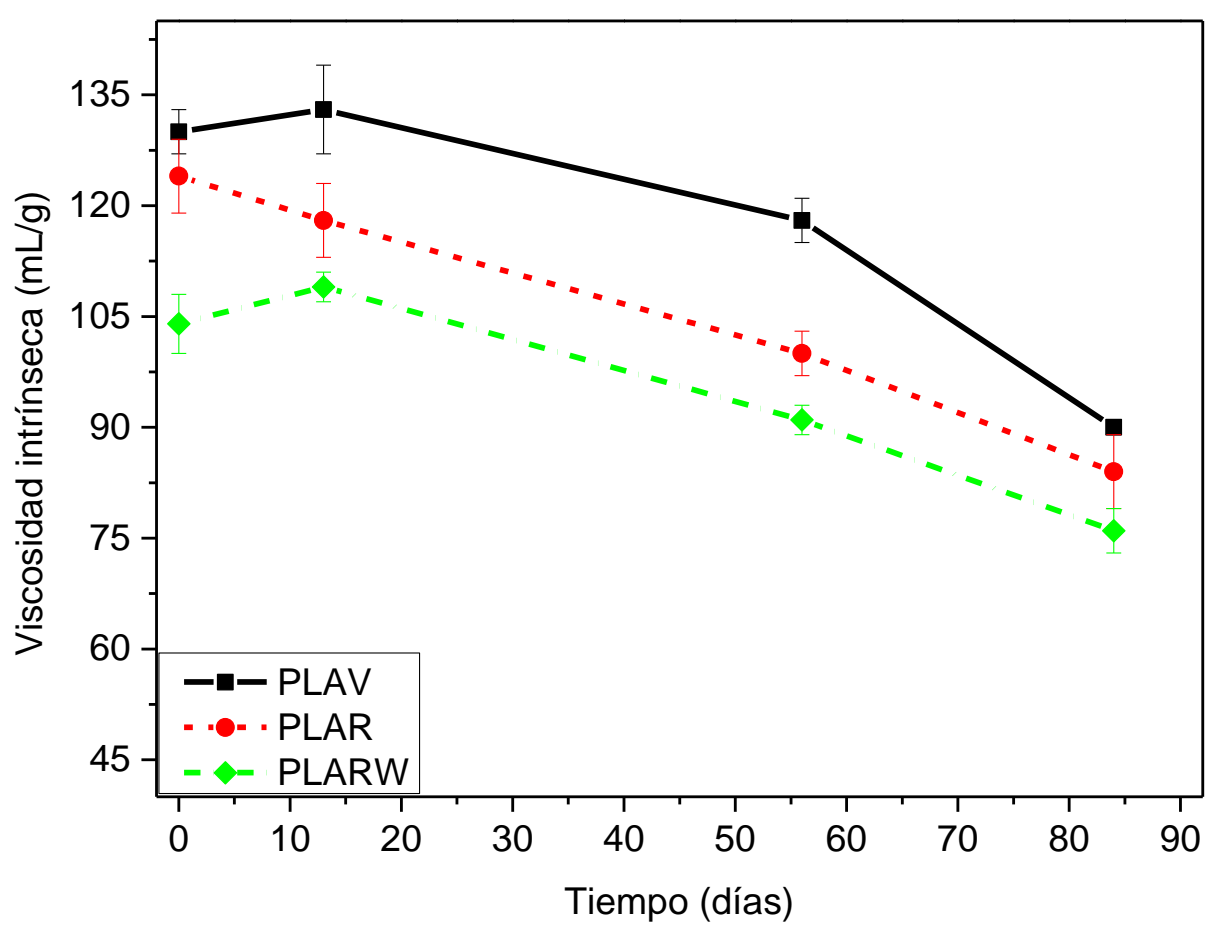

Fig. 33. Evolución de la viscosidad intrínseca, a $37^{\circ} \mathrm{C}$, del PLA virgen y sometido a diferentes procesos de reciclado.

En cuanto al comportamiento de los nanocomposites, en la Fig. 34 y la Fig. 35 se puede apreciar que, a $37{ }^{\circ} \mathrm{C}$, el comportamiento de los nanocomposites con $\mathrm{C} 30 \mathrm{y}$ haloisita, es bastante similar al del PLA puro, ya que no se observan diferencias importantes en la evolución de la viscosidad de los diferentes materiales. 
Capítulo 5. Efecto del reciclado en la degradación hidrolítica

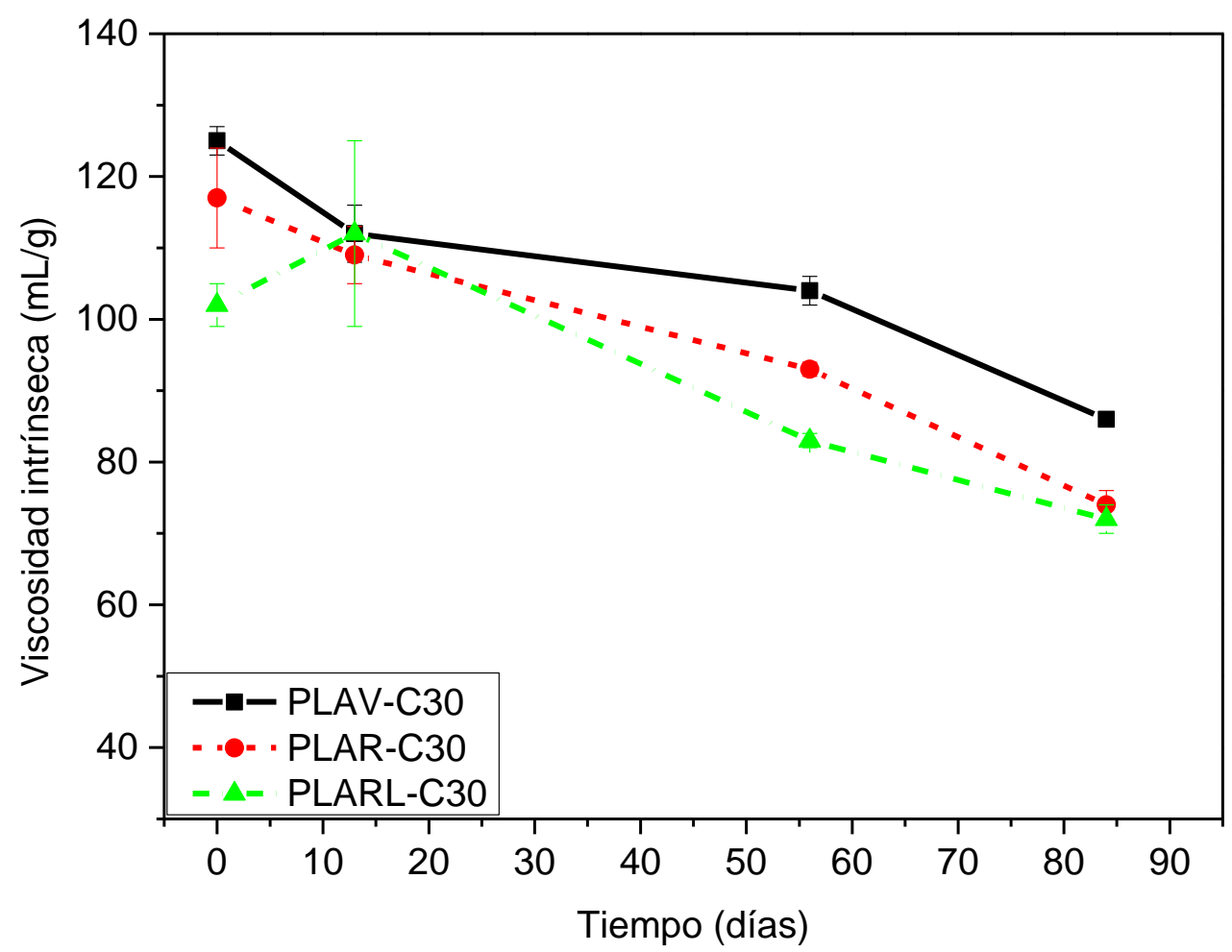

Fig. 34. Evolución de la viscosidad intrínseca, a $37^{\circ} \mathrm{C}$, del nanocomposite PLA-C30 sometido a diferentes procesos de reciclado.

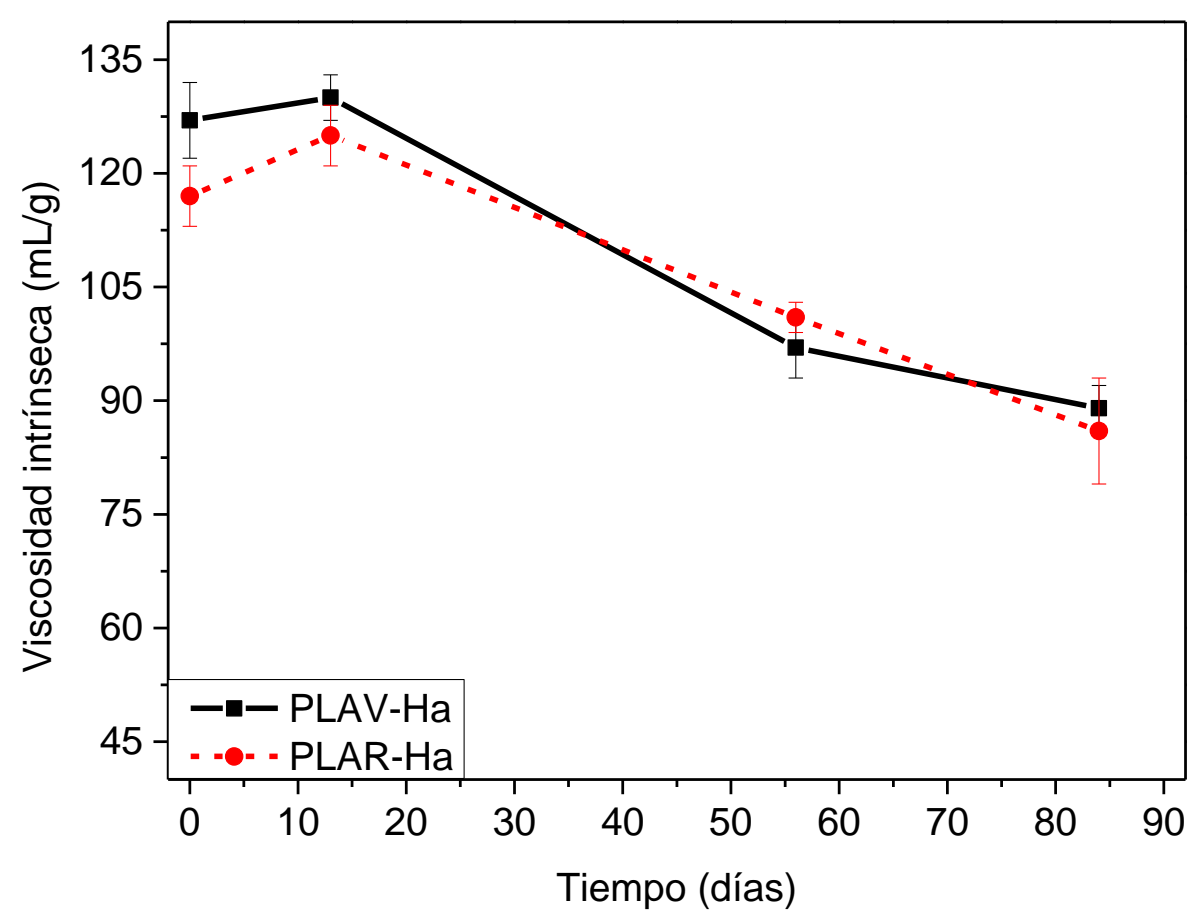

Fig. 35. Evolución de la viscosidad intrínseca, a $37{ }^{\circ} \mathrm{C}$, del nanocomposite PLA-Ha sometido a diferentes procesos de reciclado. 
Además de los ensayos de inmersión a $37^{\circ} \mathrm{C}$, la degradación hidrolítica también fue estudiada, para algunos materiales, a $58^{\circ} \mathrm{C}$. En la Tabla 9 se puede observar que la degradación del PLA, a esta temperatura, es bastante más rápida que a $37{ }^{\circ} \mathrm{C}$, con descensos de hasta $70 \%$ después de sólo 13 días de inmersión, coincidiendo con las observaciones hechas mediante espectroscopía UV-Vis. Un comportamiento similar ha sido publicado en la literatura, ya que Fukushima et al. reportan un descenso de hasta $90 \%$ en el peso molecular después de 8 semanas de inmersión a $58{ }^{\circ} \mathrm{C}$ [48]. Esta mayor degradación podría ser atribuida tanto a la mayor absorción de agua, como a un aumento de la velocidad de la hidrólisis del PLA, ambas como consecuencia del incremento en la temperatura de inmersión.

Tabla 9. Evolución de la viscosidad intrínseca de los diferentes materiales sumergidos a $58^{\circ} \mathrm{C}$.

\begin{tabular}{ccc}
\hline Material & Tiempo (días) & Viscosidad intrínseca $(\mathrm{mL} / \mathrm{g})$ \\
\hline \multirow{2}{*}{ PLAV } & 0 & $130 \pm 3$ \\
& 6 & $82 \pm 1$ \\
PLAR & 13 & $40 \pm 1$ \\
& 0 & $124 \pm 5$ \\
PLAV-C30 & 6 & $78 \pm 1$ \\
& 13 & $37 \pm 1$ \\
\hline PLAR-C30 & 0 & $125 \pm 2$ \\
& 6 & $81 \pm 2$ \\
& 13 & $38 \pm 1$ \\
& 0 & $117 \pm 7$ \\
PLAV-Ha & 6 & $76 \pm 4$ \\
& 13 & $33 \pm 5$ \\
& 0 & $127 \pm 5$ \\
PLAR-Ha & 6 & $100 \pm 2$ \\
& 13 & $62 \pm 1$ \\
& 0 & $88 \pm 1$ \\
& 6 & $41 \pm 1$ \\
\hline
\end{tabular}

En cuanto al comportamiento de los nanocomposites sumergidos a $58{ }^{\circ} \mathrm{C}$, en la Tabla 9 se observa que el nanocomposite PLA-C30 tiene un comportamiento muy similar al del PLA puro. Sin embargo, el nanocomposite con haloisita muestra una degradación menor que el resto de los materiales estudiados. Esta disminución en la velocidad de la degradación hidrolítica puede ser explicada por la naturaleza química de la haloisita sin modificar. De hecho, algunos autores como Burg y Shalabi [24] y Yu et al. [45] reportan que la haloisita sin modificar tiene un carácter básico, que no existe en la arcilla C30 modificada orgánicamente. Teniendo en cuenta que los grupos - $\mathrm{COOH}$ generados en las primeras etapas de la degradación ejercen aparentemente un efecto autacatalítico en la 
degradación hidrolítica posterior de las cadenas de poliéster, el carácter básico de la superficie de la haloisita es importante ya que le permite reaccionar con los grupos $\mathrm{COOH}$, reducir la autocatálisis y así ralentizar el proceso de degradación del polímero.

En líneas generales, la evolución de la viscosidad intrínseca de los materiales vírgenes y reciclados, tanto a 37 como a $58^{\circ} \mathrm{C}$, es muy similar. Estos resultados sugieren que la estabilidad del PLA, y sus nanocomposites, frente a la degradación hidrolítica no se ve afectada de manera significativa por los diferentes procesos de reciclado, lo cual es muy importante en el campo del envasado de alimentos.

\subsection{Cambios estructurales durante la degradación hidrolítica}

Como se ha observado anteriormente, durante la inmersión a diferentes temperaturas el PLA sufre un proceso de degradación hidrolítica, que podría ocasionar cambios estructurales en el PLA. Concretamente, sería muy importante la formación de estructuras cristalinas, que podría favorecerse por la presencia de cadenas poliméricas más cortas, generadas en los procesos de degradación. Esta cristalización alteraría las propiedades mecánicas, ópticas y de barrera del material. Dichas estructuras cristalinas presentan varias formas, tal y como se ha mencionado anteriormente, siendo las formas $\alpha$ y su variante $\alpha$ ' las más importantes. Es importante destacar también que la proporción entre estos dos polimorfos tiene un efecto importante sobre las propiedades mecánicas y de barrera del PLA.

Debido al importante papel que juega la presencia de diferentes estructuras cristalinas, es interesante estudiar los cambios estructurales del PLA durante su inmersión en el tampón de fosfatos a diferentes temperaturas, y el efecto del reciclado en dichos cambios estructurales. Para ello se realizaron ensayos de espectroscopía FTIR, XRD y DSC.

Por una parte, en la Fig. 36 se muestran los espectros del PLAV antes y después de 84 días de inmersión a $37^{\circ} \mathrm{C}$, así como los espectros del PLAR y el PLARL sometidos al mismo proceso de degradación a $37^{\circ} \mathrm{C}$. Se puede observar que no existen diferencias importantes en los espectros de las diferentes muestras, lo que parece indicar que a pesar del 30\% de reducción de la viscosidad intrínseca, los cambios en la naturaleza química y en la estructura del PLA son bastante pequeños, y no se ven afectados por el reciclado. 


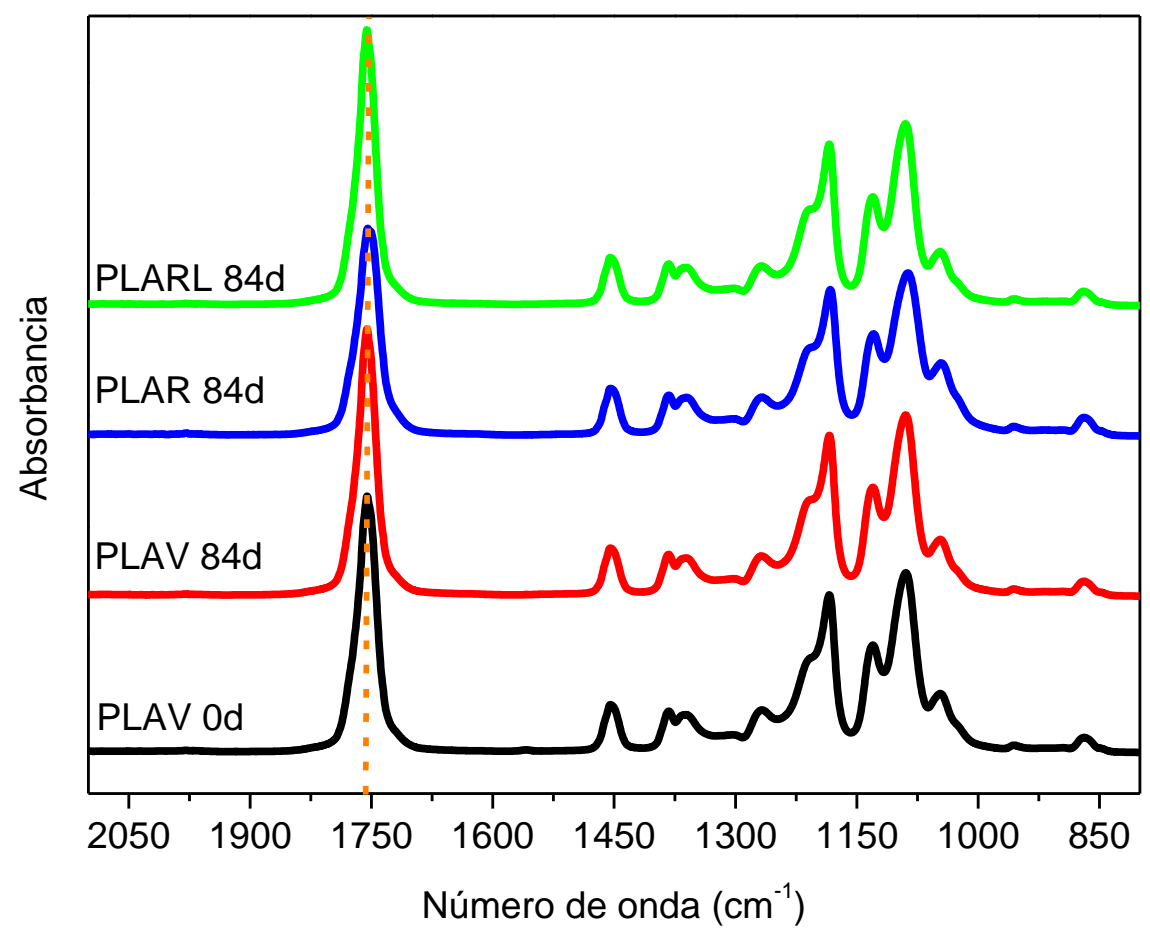

Fig. 36. Espectros FTIR del PLAV sin sumergir, y del PLAV, PLAR y PLARL después de 84 días de inmersión a $37^{\circ} \mathrm{C}$.

Por otra parte, el comportamiento del nanocomposite PLA-C30 es diferente. En la Fig. 37 se observa que, aunque los espectros de los diferentes materiales son muy parecidos, existe una pequeña diferencia entre 970 y $900 \mathrm{~cm}^{-1}$, concretamente, se puede apreciar un pequeño aumento de la banda de absorción localizada a $920 \mathrm{~cm}^{-1}$. Dicha banda ha sido relacionada previamente con la conformación helicoidal $10_{3}$ de las cadenas poliméricas, característica de las diferentes formas cristalinas del PLA [33]. 


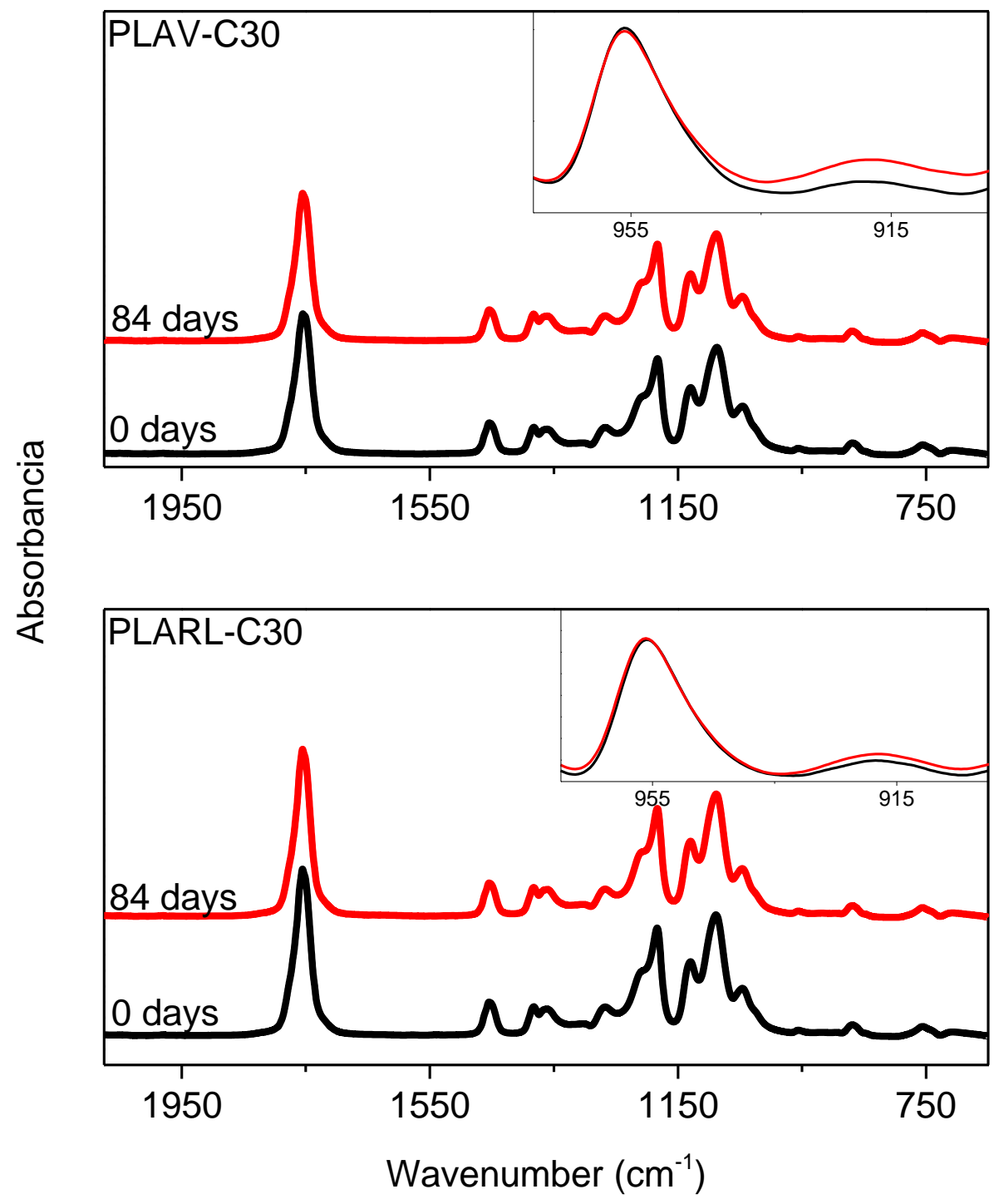

Fig. 37. Espectros del PLAV-C30 y el PLAR-C30 antes y después de 84 días de inmersión a $37^{\circ} \mathrm{C}$.

Además de los ensayos de espectroscopía FTIR, se realizaron difractogramas del PLA puro y del nanocompuesto PLA-C30 durante la inmersión a $37{ }^{\circ} \mathrm{C}$. La Fig. 38 muestra los difractogramas del PLA, sometido a diferentes procesos de reciclado, antes y después de 84 días de inmersión a $37^{\circ} \mathrm{C}$. Se puede observar el amplio pico de difracción correspondiente al PLA amorfo; sin embargo, después de 84 días de inmersión se puede apreciar la aparición de un pequeño pico de difracción alrededor de $17^{\circ}$, que ha sido relacionado en diferentes estudios con el plano (110)/(200) del PLA cristalino $[35,96,118]$. Este comportamiento es más pronunciado en el material sometido al lavado intenso.

Esta diferencia podría ser debida a que, tal como se observa en la evolución de la viscosidad intrínseca, después de 84 días el PLARL tiene cadenas poliméricas más cortas que el PLAV, las cuales tienen una mayor movilidad y por lo tanto son capaces de formar estructuras cristalinas con una mayor facilidad. 

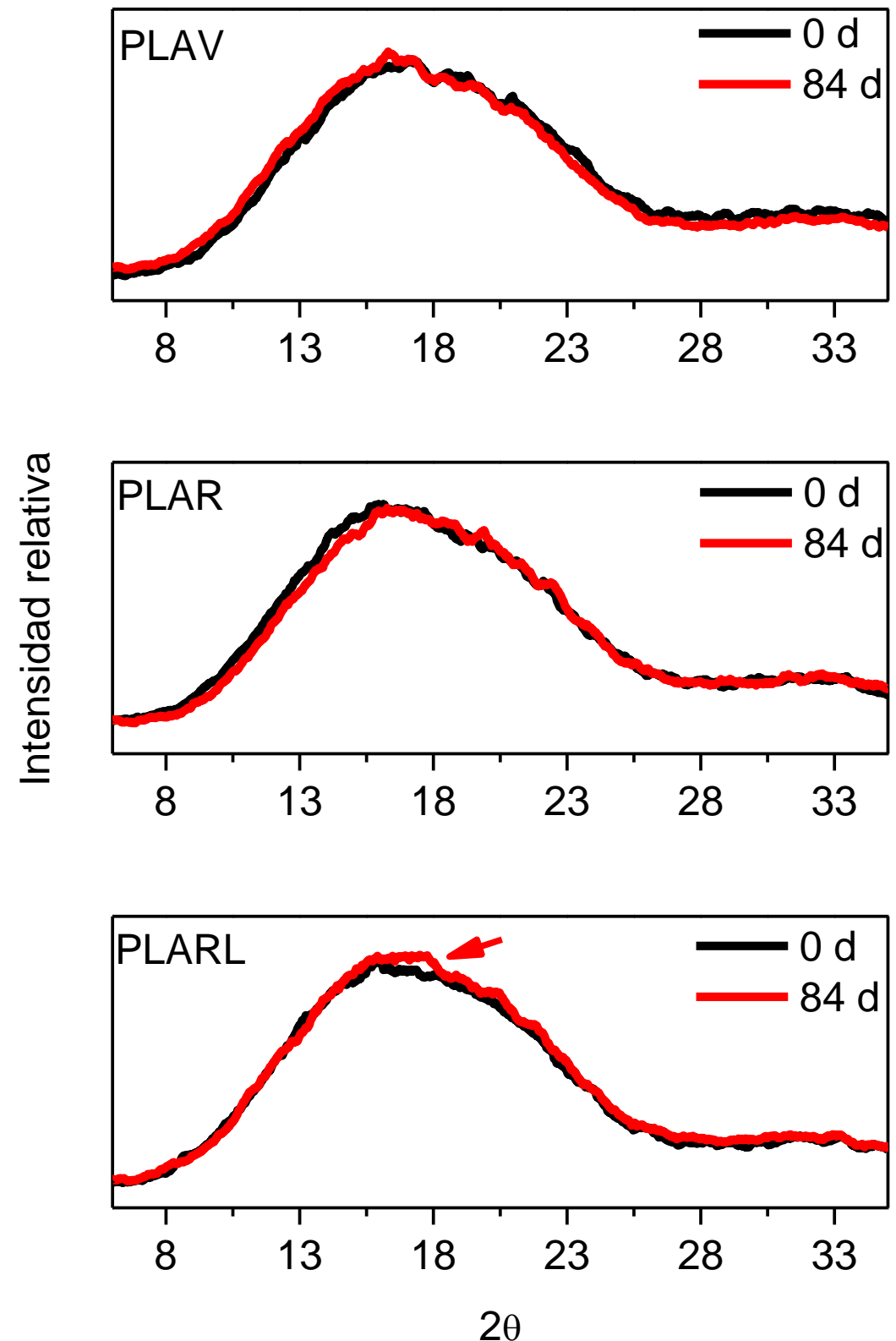

Fig. 38. Difractogramas del PLA, sometido a diferentes procesos de reciclado, sumergido a $37^{\circ} \mathrm{C}$.

En cuanto al comportamiento de los nanocomposites PLA-C30, la Fig. 38 muestra que, a diferencia de lo ocurrido con el PLA puro, tanto el nanocomposite virgen como los reciclados muestran un pequeño pico de difracción después de estar sumergido durante 84 días a $37^{\circ} \mathrm{C}$. Estos resultados coinciden con los observados mediante DSC y FTIR, en los que se puede apreciar que después de 84 días de inmersión todas las muestras presentan un ligero aumento de la cristalinidad. 

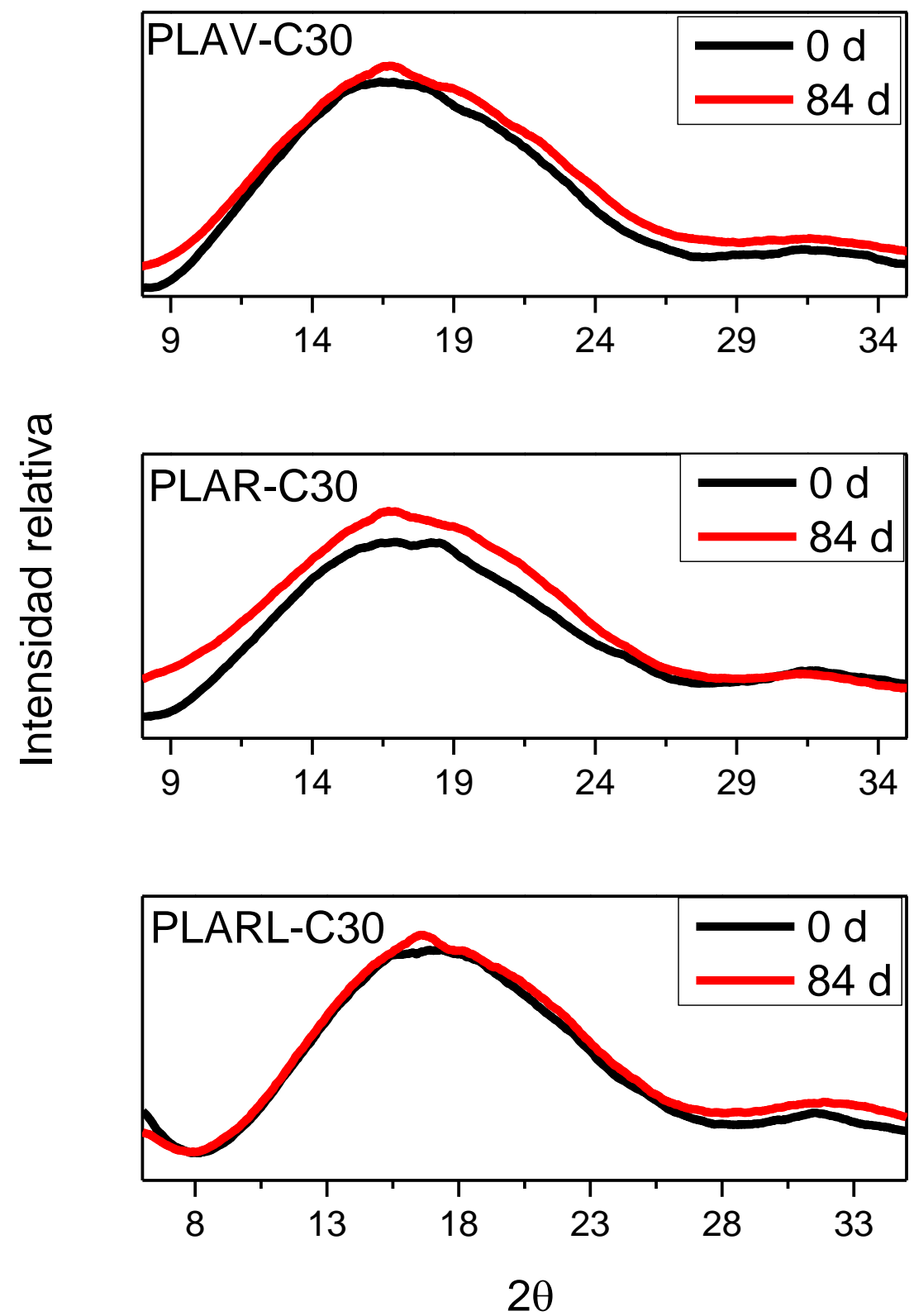

Fig. 39. Difractogramas del nanocomposite PLA-C30, sometido a diferentes procesos de reciclado, sumergido a $37^{\circ} \mathrm{C}$.

Con la finalidad de profundizar más en el estudio de los cambios estructurales ocurridos a $37^{\circ} \mathrm{C}$ en el PLA y sus nanocomposites, se realizaron ensayos de DSC de los diferentes materiales. En la Fig. 40 y en la Tabla 10 se recogen los resultados obtenidos del primer barrido de calentamiento del PLA puro sometido a diferentes procesos de reciclado, a diferentes tiempos de inmersión. 

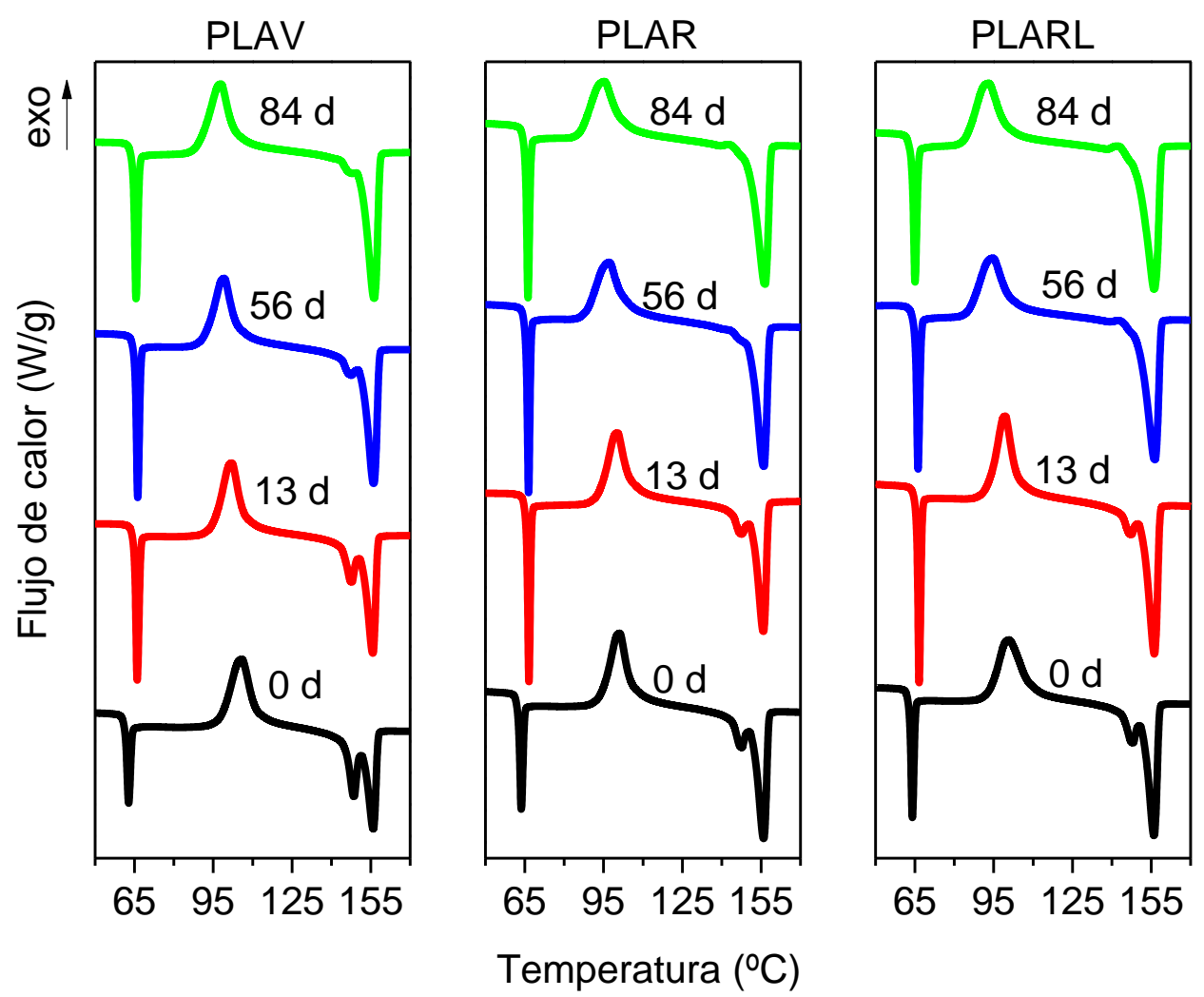

Fig. 40. Primer barrido de calentamiento del PLAV, PLAR y PLARL sumergido a 37 ${ }^{\circ} \mathrm{C}$.

En primer lugar, se puede observar que, en todos los casos, los barridos de DSC presentan las transiciones térmicas características del PLA, una transición vítrea alrededor de $60{ }^{\circ} \mathrm{C}$, una cristalización en frío alrededor de los $100{ }^{\circ} \mathrm{C}$ y una fusión por encima de los $140^{\circ} \mathrm{C}$. Sin embargo, en la Fig. 40 se observa la aparición de un pico endotérmico acompañando a la transición vítrea del PLA. Este pico ha sido atribuido al envjecimiento físico del PLA, que consiste en la densificación de las regiones amorfas del polímero, como resultado de la reorganización molecular, que conduce a las variables termodinámicas hacia su valor de equilibirio [31].

En segundo lugar, tanto la Fig. 40 como la Tabla 10 reflejan una disminución de la $T_{c c}$ del PLA. Este resultado puede ser explicado porque la presencia de cadenas poliméricas más cortas, consecuencia de la degradación hidrolítica, favorece la cristalización en frío y ocasiona un descenso de su temperatura característica. Badía et al. [13] reportaron resultados similares al estudiar la degradación hidrolítica de PLA reprocesado. En cuanto al efecto del reciclado, se puede observar en la Tabla 10 que, más allá de las diferencias iniciales en los valores de $T_{c c}$, el descenso en la temperatura de cristalización es similar en el material virgen y en los diferentes reciclados, incluso en el caso del material sometido al proceso de lavado. Los resultados obtenidos concuerdan con los observados en las medidas de viscosidad intrínseca, en los que se encontró que 
los diferentes procesos de reciclado no afectan de forma significativa la velocidad de la degradación hidrolítica del PLA.

En tercer lugar, en la Fig. 40 se pueden apreciar cambios en las endotermas de fusión del PLA, tanto virgen como reciclado. A medida que aumenta el tiempo de inmersión, el pico de fusión de menor temperatura pierde relevancia hasta practicamente desaparecer después de 84 días de inmersión. Este cambio se debe también a la degradación hidrolítica del PLA, ya que las cadenas más cortas tienen mayor facilidad para reorganizarse y formar cristales más perfectos, los cuales funden a una mayor temperatura [19]. Es conveniente resaltar que estos cambios parecen ser ligeramente más pronunciados en los materiales reciclados, lo que podría ser explicado por el menor peso molecular de estas muestras. Además, es importante destacar que tanto el PLAR como el PLARL, sumergidos durante 84 días, presentan un pequeño pico exotérmico justamente antes de la endoterma de fusión. Este pico también fue observado por Zhang et al. [119] en muestras de PLA cristalizado a diferentes temperaturas, y ha sido atribuido a la reorganización de la fase $\alpha$ ' en la fase $\alpha$. Nuestros resultados sugieren que la inmersión a $37^{\circ} \mathrm{C}$ ocasiona la formación de la forma $\alpha$, o de al menos una mezcla entre las formas $\alpha$ ' y $\alpha$.

Finalmente, en la Tabla 10 se puede observar que en todas las muestras hay un aumento en el grado de cristalinidad con el tiempo de inmersión. Como ya se ha mencionado anteriormente, esta cristalización se debe tanto a la degradación hidrolítica como al efecto plastificante del medio de inmersión en el PLA, ya que ambos promueven una mayor movilidad de las cadenas poliméricas, facilitando su reorganización en estructuras cristalinas. Sin embargo, a pesar de que en todos los materiales se observa un aumento de la cristalinidad, este efecto parece ser mayor en el PLAR y el PLARL. Esto se debe, probablemente, al menor peso molecular de estos materiales, lo que indica que poseen cadenas más cortas que cristalizan con mayor facilidad. Estos resultados confirman las observaciones realizadas mediante XRD, donde el PLA reciclado presentaba una cristalinidad ligeramente mayor.

Tabla 10. Evolución de las transiciones térmicas durante la inmersión a $37^{\circ} \mathrm{C}$ del PLA sometido a diferentes procesos de reciclado.

\begin{tabular}{ccccccc}
\hline Material & $\begin{array}{c}\text { Tiempo } \\
(\mathbf{d i ́ a s})\end{array}$ & $\begin{array}{c}\mathbf{T}_{\mathbf{c c}} \\
\left({ }^{\mathbf{}} \mathbf{C}\right)\end{array}$ & $\begin{array}{c}\mathbf{T}_{\text {fus }} \\
\left({ }^{\mathbf{}} \mathbf{C}\right)\end{array}$ & $\begin{array}{c}\Delta \mathbf{H}_{\text {crist }} \\
(\mathbf{J} / \mathbf{g})\end{array}$ & $\begin{array}{c}\Delta \mathbf{H}_{\text {fus }} \\
(\mathbf{J} / \mathbf{g})\end{array}$ & $\begin{array}{c}\mathbf{X}_{\mathbf{C}} \\
(\mathbf{\%})\end{array}$ \\
\hline \multirow{3}{*}{ PLAV } & 0 & 106,0 & $148,6-155,9$ & 27,1 & 27,6 & 1 \\
& 13 & 102,1 & $147,8-157,8$ & 27,2 & 28,2 & 1 \\
& 56 & 99,2 & $147,6-156,1$ & 27,6 & 29,5 & 2 \\
PLAR & 84 & 98,0 & 156,3 & 29,1 & 33,1 & 4 \\
& 0 & 101,2 & $147,7-156,0$ & 27,6 & 28,3 & 1 \\
& 13 & 100,4 & $147,7-155,9$ & 27,4 & 28,3 & 1 \\
& 56 & 97,1 & 156,1 & 26,1 & 30,8 & 5 \\
& 84 & 95,2 & 16,5 & 25,0 & 32,4 & 8 \\
\hline
\end{tabular}




\begin{tabular}{lcccccc}
\hline & 0 & 100,9 & $148,1-156,1$ & 28,0 & 29,8 & 2 \\
\multirow{3}{*}{ PLARL } & 13 & 99,5 & $147,2-157,2$ & 30,1 & 31,6 & 2 \\
& 56 & 94,7 & 156,4 & 27,4 & 32,9 & 6 \\
& 84 & 93,1 & 156 & 28,6 & 35,6 & 8 \\
\hline
\end{tabular}

En cuanto al comportamiento de los nanocomposites PLA-C30, los resultados de DSC de dichas muestras se encuentran recogidos en la Fig. 41 y la Tabla 11. Es importante destacar que, en líneas generales, el comportamiento de los nanocomposites PLA-C30 es similar al del PLA puro, observándose un descenso progresivo de la temperatura de cristalización en frío, un aumento de la importancia del pico de fusión de alta temperatura y un ligero incremento del grado de cristalinidad.
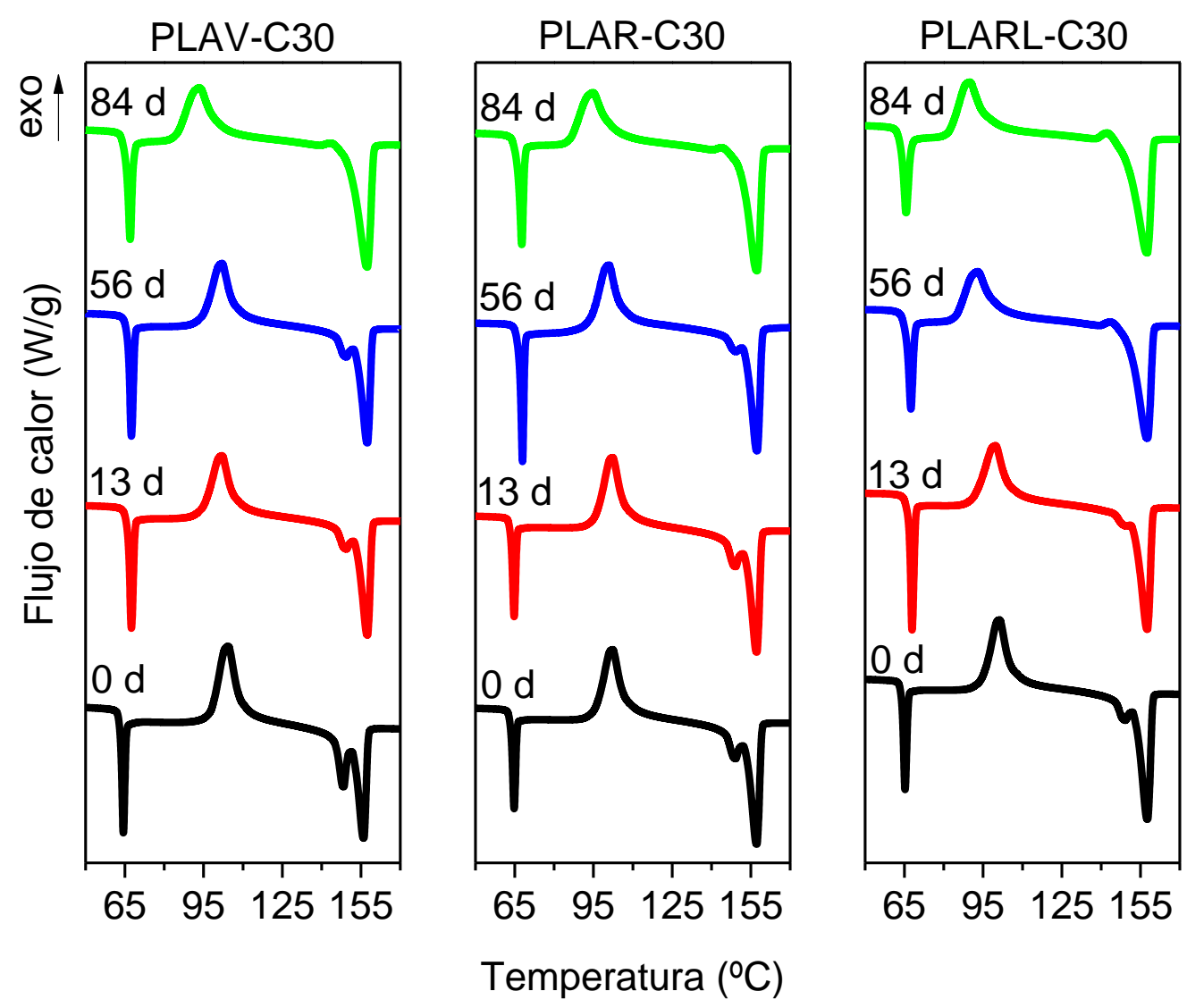

Fig. 41. Primer barrido de calentamiento del nanocomposite PLA-C30, sometido a diferentes procesos de reciclado, sumergido a $37^{\circ} \mathrm{C}$.

Sin embargo, la presencia de la arcilla ocasiona algunos cambios en la evolución de la cristalinidad de los nanocomposites. Mientras que en el PLA puro el material virgen desarrollaba una menor cristalinidad que los materiales reciclados, en el nanocomposite los 3 materiales (PLAV-C30, PLAR-C30 y PLARL-C30) muestran crecimiento similar de la cristalinidad y el pico exotérmico característico de la transformación $\alpha^{\prime} \rightarrow \alpha$. Esta 
mayor cristalización en los nanocomposites con C30 puede estar relacionada los menores valores de viscosidad del nanocomposite PLA-C30 en comparación con el PLA puro, lo que indica la presencia de cadenas más cortas que cristalizan más fácilmente.

Tabla 11. Evolución de las transiciones térmicas durante la inmersión a $37^{\circ} \mathrm{C}$ del nanocomposite PLA-C30 sometido a diferentes procesos de reciclado.

\begin{tabular}{|c|c|c|c|c|c|c|}
\hline Material & $\begin{array}{c}\text { Tiempo } \\
\text { (días) }\end{array}$ & $\begin{array}{c}\mathbf{T}_{\mathrm{cc}} \\
\left({ }^{\circ} \mathrm{C}\right)\end{array}$ & $\begin{array}{l}T_{\text {fus }} \\
\left({ }^{\circ} \mathrm{C}\right)\end{array}$ & $\begin{array}{c}\Delta H_{\text {crist }} \\
(\mathrm{J} / \mathrm{g})\end{array}$ & $\begin{array}{r}\Delta H_{\text {fus }} \\
(\mathrm{J} / \mathrm{g})\end{array}$ & $\begin{array}{c}\mathbf{X}_{\mathrm{C}} \\
(\%)\end{array}$ \\
\hline \multirow{4}{*}{ PLAV-C30 } & 0 & 104,4 & $148,29-156,11$ & 26,8 & 27,8 & 1 \\
\hline & 13 & 102,0 & $149,23-157,43$ & 24,1 & 25,5 & 2 \\
\hline & 56 & 95,1 & 155,86 & 24,9 & 30,1 & 6 \\
\hline & 84 & 93,4 & 157,44 & 23,5 & 30,4 & 7 \\
\hline \multirow{4}{*}{ PLAR-C30 } & 0 & 102,3 & $149,22-157,22$ & 26,0 & 27,2 & 1 \\
\hline & 13 & 100,8 & $149,33-157,37$ & 25,8 & 26,8 & 1 \\
\hline & 56 & 94,7 & 157,39 & 24,0 & 28,9 & 5 \\
\hline & 84 & 90,6 & 157,52 & 23,7 & 31,5 & 8 \\
\hline \multirow{4}{*}{ PLARL-C30 } & 0 & 101,3 & $149,26-157,61$ & 27,1 & 28,1 & 1 \\
\hline & 13 & 99,7 & $149,54-157,56$ & 25,1 & 27,9 & 3 \\
\hline & 56 & 92,9 & 157,69 & 24,1 & 28,5 & 5 \\
\hline & 84 & 89,9 & 157,59 & 24,9 & 31,1 & 7 \\
\hline
\end{tabular}

El nanocomposite PLA-Ha, tal como se muestra en la Fig. 42 y en la Tabla 12 muestra un comportamiento general análogo al del PLA puro y al del nanocomposite con C30. Se puede observar que la degradación hidrolítica ocasiona un descenso en la $T_{c c}$, la desaparición progresiva del pico de fusión de baja temperatura y un aumento de la cristalinidad. Sin embargo, la evolución de los valores de cristalinidad es más parecida a la del PLA puro que al nanocompuesto con C30, ya que el PLAV-Ha presenta un incremento ligeramente menor que el PLAR-Ha. Esta diferencia podría explicarse si se considera la menor degradación del PLAV-Ha, tal como se observó en las medidas de viscosidad, ya que las cadenas más largas tienen menos facilidad para formar estructuras cristalinas. Así mismo, algunos autores, como Lecouvet et al. [64], señalan que la presencia de nanotubos de haloisita limita la habilidad para cristalizar de la poliamida-12, por lo que la presencia de los nanotubos de arcilla podría dificultar la cristalización del PLA en nuestros nanocomposites. 


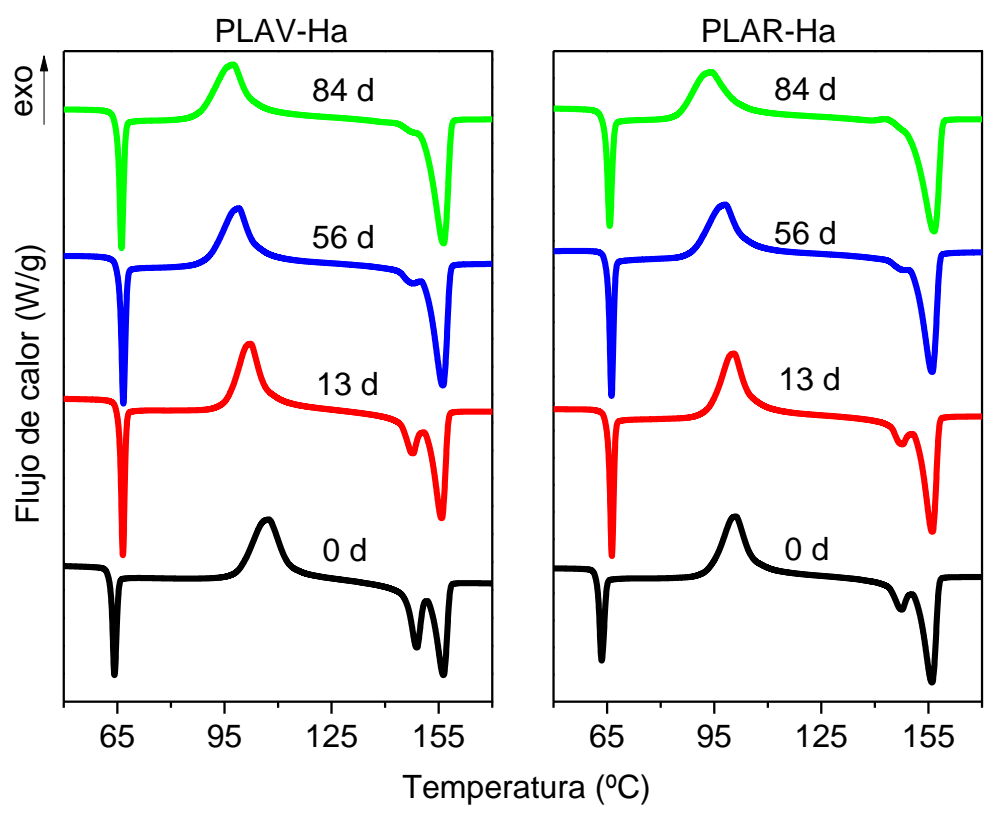

Fig. 42. Primer barrido de calentamiento del nanocomposite PLA-Ha, sometido a diferentes procesos de reciclado, sumergido a $37^{\circ} \mathrm{C}$.

Al igual que en el PLA puro, el nanocomposite reciclado, PLAR-Ha, presenta mayores valores de cristalinidad que el material virgen. Este resultado es coherente con lo observado en las medidas de viscosidad, donde la degradación del PLAR-Ha era significativamente mayor que en el PLAV-Ha.

Tal y como se ha observado, los cambios estructurales del PLA y los nanocomposites con $\mathrm{C} 30$ y haloisita sumergidos a $37^{\circ} \mathrm{C}$ son relativamente pequeños, y tienen lugar a largos tiempos de inmersión. Este resultado es muy importante desde el punto de vista de las aplicaciones de envasado de alimentos, en las que se espera que el PLA esté en contacto con líquidos y alimentos húmedos, pero durante períodos de tiempo cortos, por lo que el material reciclado podría ser utilizado en este tipo de aplicaciones.

Tabla 12. Evolución de las transiciones térmicas durante la inmersión a $37^{\circ} \mathrm{C}$ del nanocomposite PLA-Ha sometido a diferentes procesos de reciclado.

\begin{tabular}{ccccccc}
\hline Material & $\begin{array}{c}\text { Tiempo } \\
(\mathbf{d i ́ a s})\end{array}$ & $\begin{array}{c}\mathbf{T}_{\mathbf{c c}} \\
\left({ }^{\mathbf{C}} \mathbf{C}\right)\end{array}$ & $\begin{array}{c}\mathbf{T}_{\text {fus }} \\
\left({ }^{\mathbf{O}} \mathbf{C}\right)\end{array}$ & $\begin{array}{c}\Delta \mathbf{H}_{\text {crist }} \\
(\mathbf{J} / \mathbf{g})\end{array}$ & $\begin{array}{c}\Delta \mathbf{H}_{\text {fus }} \\
(\mathbf{J} / \mathbf{g})\end{array}$ & $\begin{array}{c}\mathbf{X}_{\mathbf{C}} \\
(\mathbf{\%})\end{array}$ \\
\hline \multirow{3}{*}{ PLAV-Ha } & 0 & 107,4 & $148,8-156,3$ & 27,2 & 28,4 & 1 \\
& 13 & 102,3 & $147,7-155,9$ & 27,4 & 28,4 & 1 \\
& 56 & 98,9 & $147,4-156,3$ & 27,9 & 29,4 & 2 \\
PLAR-Ha & 84 & 97,4 & 156,4 & 27,8 & 30,4 & 3 \\
& 0 & 101,1 & $147,2-156,0$ & 27,4 & 28,0 & 1 \\
& 56 & 100,5 & $147,6-156,2$ & 27,9 & 28,9 & 1 \\
& & 98,0 & 156,1 & 27,4 & 29,8 & 3
\end{tabular}


Además de los cambios a $37^{\circ} \mathrm{C}$, se estudiaron los cambios estructurales que tuvieron lugar en las muestras sumergidas a $58{ }^{\circ} \mathrm{C}$, temperatura muy próxima a la $T_{g}$ del PLA, por lo que es de esperar que los cambios estructurales sean muy importantes durante la inmersión.

La Fig. 43 muestra cuatro regiones diferentes del espectro FTIR de las diferentes muestras, antes y después de 13 días de inmersión a $58^{\circ} \mathrm{C}$. Estas regiones, (a) 3050 - 2850 $\mathrm{cm}^{-1}$, (b) $1800-1700 \mathrm{~cm}^{-1}$, (c) $1300-1100 \mathrm{~cm}^{-1}$ y (d) $980-880 \mathrm{~cm}^{-1}$ han sido consideradas debido a que diferentes estudios, realizados en PLA cristalizado térmicamente, reportan que los cambios estructurales del polímero se ven reflejados en ellas $[33,72,73,85,118,119]$.

La Fig. 43a muestra el espectro tanto del PLAV como de los nanocomposites con haloisita y C30, en la región entre 3050 y $2850 \mathrm{~cm}^{-1}$. Como se ha mencionado en la caracterización de los materiales, en el material sin sumergir las bandas ubicadas a 2995 y $2945 \mathrm{~cm}^{-1}$ corresponden a la tensión asimétrica y simétrica de los grupos $\mathrm{CH}_{3}$ respectivamente. Después de 13 días de inmersión, se observa en todos los casos un pequeño desplazamiento hacia mayores números de onda. Un comportamiento similar fue observado por Zhang et al. [118], al estudiar la cristalización térmica de PLLA seco entre 80 y $140{ }^{\circ} \mathrm{C}$. En dicho estudio reportan la aparición de nuevas interacciones dipolodipolo como consecuencia del empaquetamiento de las cadenas en la celda unitaria cristalina del PLLA, las cuales serían las responsables de la aparición de dos hombros muy débiles a 2955 y $3006 \mathrm{~cm}^{-1}$, ocasionando el desplazamiento de las bandas de tensión $\mathrm{CH}_{3}$ hacia mayores números de onda.

En la Fig. 43b se muestra la región entre 1790 y $1710 \mathrm{~cm}^{-1}$, correspondiente a la tensión de los enlaces $\mathrm{C}=\mathrm{O}$. Además de proveer información sobre la degradación del PLA, algunos estudios sugieren que esta banda puede proveer información sobre la conformación y las interacciones intra e intermoleculares de las cadenas de PLA [72,84]. Por lo tanto, a partir de los resultados obtenidos en esta región del espectro se puede obtener importante información relativa a la cristalización del PLA durante la inmersión a $58^{\circ} \mathrm{C}$. Se puede observar que el material inicial presenta una banda de absorción ancha, característica del PLA en estado amorfo. Sin embargo, después de 13 días de inmersión, se observa en todas las muestras algunos cambios. En primer lugar, hay un estrechamiento de la banda de absorción, que podría ser consecuencia de un aumento del orden molecular en las regiones cristalinas del polímero [17]. En segundo lugar, hay un incremento en la intensidad y un desplazamiento de la banda hacia números de onda más altos. Este comportamiento también ha sido observado en diferentes estudios con PLA seco, cristalizado térmicamente, y ha sido atribuido a la aparición de nuevas bandas de absorción, correspondientes a las formas cristalinas $\alpha$ y $\alpha^{\prime}[73,118]$. 


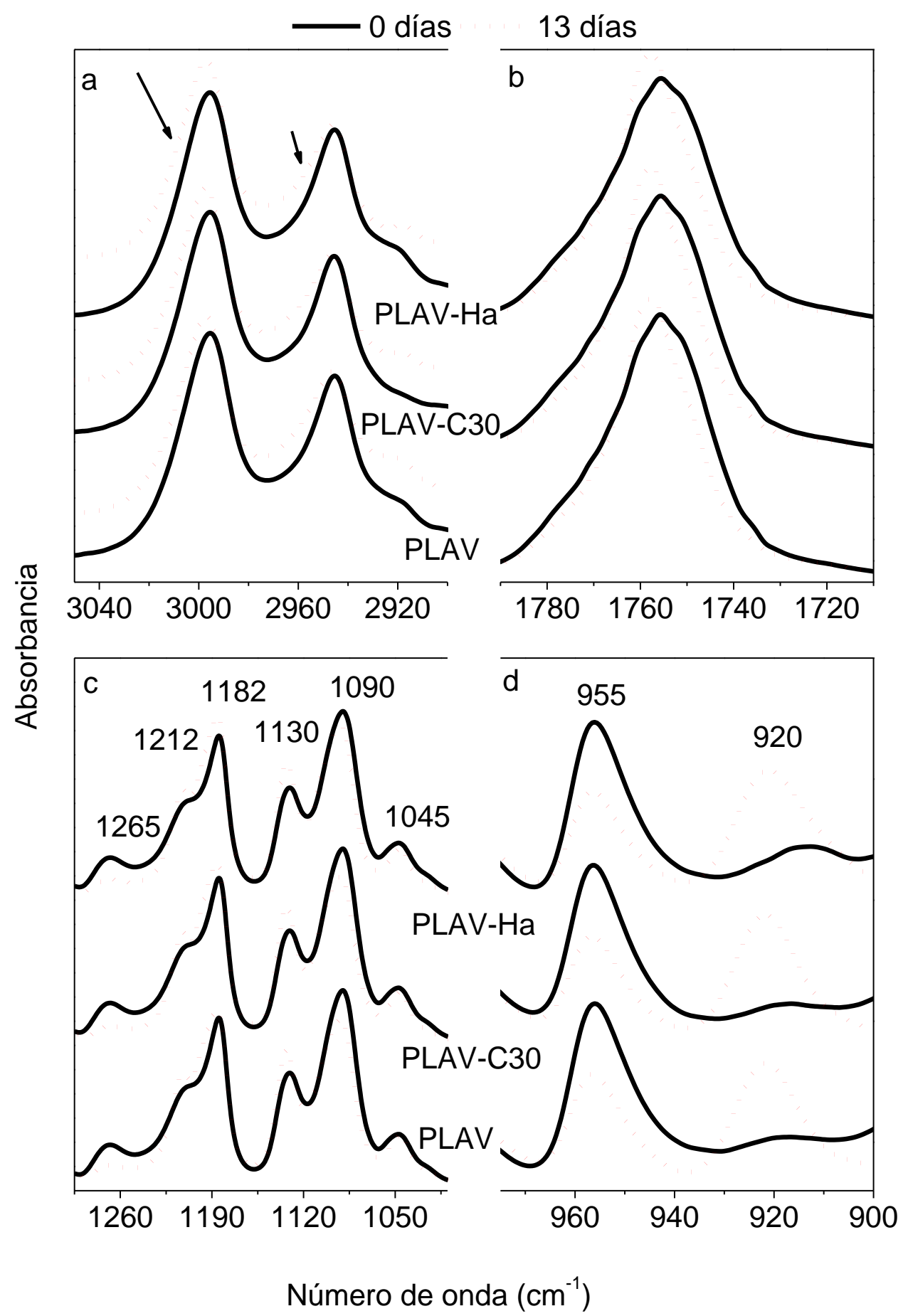

Fig. 43. Espectros FTIR de las diferentes muestras antes y después de 13 días de inmersión a $58^{\circ} \mathrm{C}$.

La Fig. 43c muestra los cambios que han tenido lugar en la región entre 1300 y $1000 \mathrm{~cm}^{-1}$, entre los que se encuentran un aumento de la intensidad de las bandas ubicadas a 1212,1182 y $1130 \mathrm{~cm}^{-1}$ y un descenso en la intensidad de las bandas localizadas a 1265 y $1090 \mathrm{~cm}^{-1}$. Las bandas ubicadas a 1212 y $1182 \mathrm{~cm}^{-1}$ han sido relacionadas con la formación de estructuras cristalinas en el PLA, durante el recocido a temperaturas altas, por lo que el crecimiento observado indica la cristalización del PLA durante la inmersión. 
Así mismo, el descenso en la intensidad de las bandas de 1265 y $1090 \mathrm{~cm}^{-1}$ ha sido relacionado, en diferentes estudios con PLA cristalizado térmicamente, con la disminución de la proporción de las regiones amorfas en el PLA [72,84,85].

Finalmente, en la Fig. 43d se muestra el intervalo entre 980 y $900 \mathrm{~cm}^{-1}$, en el cual aparecen las diferencias más significativas. Como se ha mencionado en secciones anteriores, las bandas de esta zona han sido asignadas al acoplamiento de la tensión del esqueleto $\mathrm{C}-\mathrm{C}$ y del balanceo del grupo $\mathrm{CH}_{3}$. Además, se sabe que la banda a $920 \mathrm{~cm}^{-1}$ está relacionada con las formas $\alpha$ y $\alpha$ ' cristalinas del PLLA, mientras que la banda de 956 $\mathrm{cm}^{-1}$ corresponde a las zonas amorfas. Los tres materiales estudiados muestran un incremento de la banda a $920 \mathrm{~cm}^{-1}$, junto con un descenso en la intensidad de la banda de $956 \mathrm{~cm}^{-1}$. Estos cambios, en conjunto con los observados en las otras regiones del espectro FTIR revelan una cristalización importante del PLA durante su inmersión a $58^{\circ} \mathrm{C}$. Esta transformación a una temperatura notablemente inferior a las utilizadas para cristalizar PLA seco [72], las cuales se encuentran entre 80 y $130^{\circ} \mathrm{C}$, indica que la presencia de agua favorece la cristalización del PLA. Por una parte, la degradación del polímero, que ya ha sido observada mediante medidas de viscosidad intrínseca, genera cadenas cortas que pueden reorganizarse en estructuras cristalinas más fácilmente. Por otra parte, el agua actúa también como un plastificante, que facilita el movimiento de las cadenas y su organización en estructuras cristalinas,

Varios investigadores han conseguido estimar el grado de cristalinidad mediante espectroscopía FTIR, utilizando las bandas localizadas en la región entre 970 y $850 \mathrm{~cm}^{-1}$. Por ejemplo, Meaurio et al. [72] han utilizado el área de la banda a $955 \mathrm{~cm}^{-1}$, antes y después del proceso de cristalización, para calcular el grado de cristalinidad del PLA (Ec. 1). En este trabajo se utilizó el mismo procedimiento, y los resultados obtenidos se recogen en la Tabla 13.

Tabla 13. Valores de cristalinidad estimados mediante FTIR y XRD de las diferentes muestras sumergidas a $58^{\circ} \mathrm{C}$.

\begin{tabular}{ccccccc}
\hline \multicolumn{3}{c}{$\boldsymbol{X}_{\boldsymbol{C}}(\mathrm{FTIR})$} & & \multicolumn{3}{c}{$\boldsymbol{X}_{\boldsymbol{C}}$ (XRD) } \\
\hline $\begin{array}{c}\text { Tiempo } \\
\text { (días) }\end{array}$ & PLAV & PLAV-C30 & PLAV-Ha & PLAV & PLAV-C30 & PLAV-Ha \\
\hline 2 & 38 & 36 & 27 & 45 & 45 & 34 \\
6 & 42 & 42 & 33 & 50 & 50 & 38 \\
13 & 48 & 48 & 34 & 60 & 60 & 45 \\
\hline
\end{tabular}

También se han realizado difractogramas de las diferentes muestras, para obtener información sobre la formación de estructuras cristalinas en el PLA durante la inmersión a $58^{\circ} \mathrm{C}$. Los resultados obtenidos se muestran en la Fig. 44, en la que se puede observar que, durante la inmersión, todos los materiales pasan de mostrar el halo característico del PLA amorfo, a mostrar tres picos de reflexión ubicados a 15,0; 16,0 y 19,0 ${ }^{\circ}$. De acuerdo 
a la literatura, dichos picos corresponden a los planos (010), (110/200) y (203) del PLA cristalino [35,96,118]. Estos resultados, al igual que los obtenidos mediante espectroscopía FTIR, indican una cristalización importante del PLA durante la inmersión a $58{ }^{\circ} \mathrm{C}$. A partir de estas medidas se puede calcular la cristalinidad de los diferentes materiales empleando la Ec. 4. Los resultados obtenidos se encuentran resumidos en la Tabla 13.

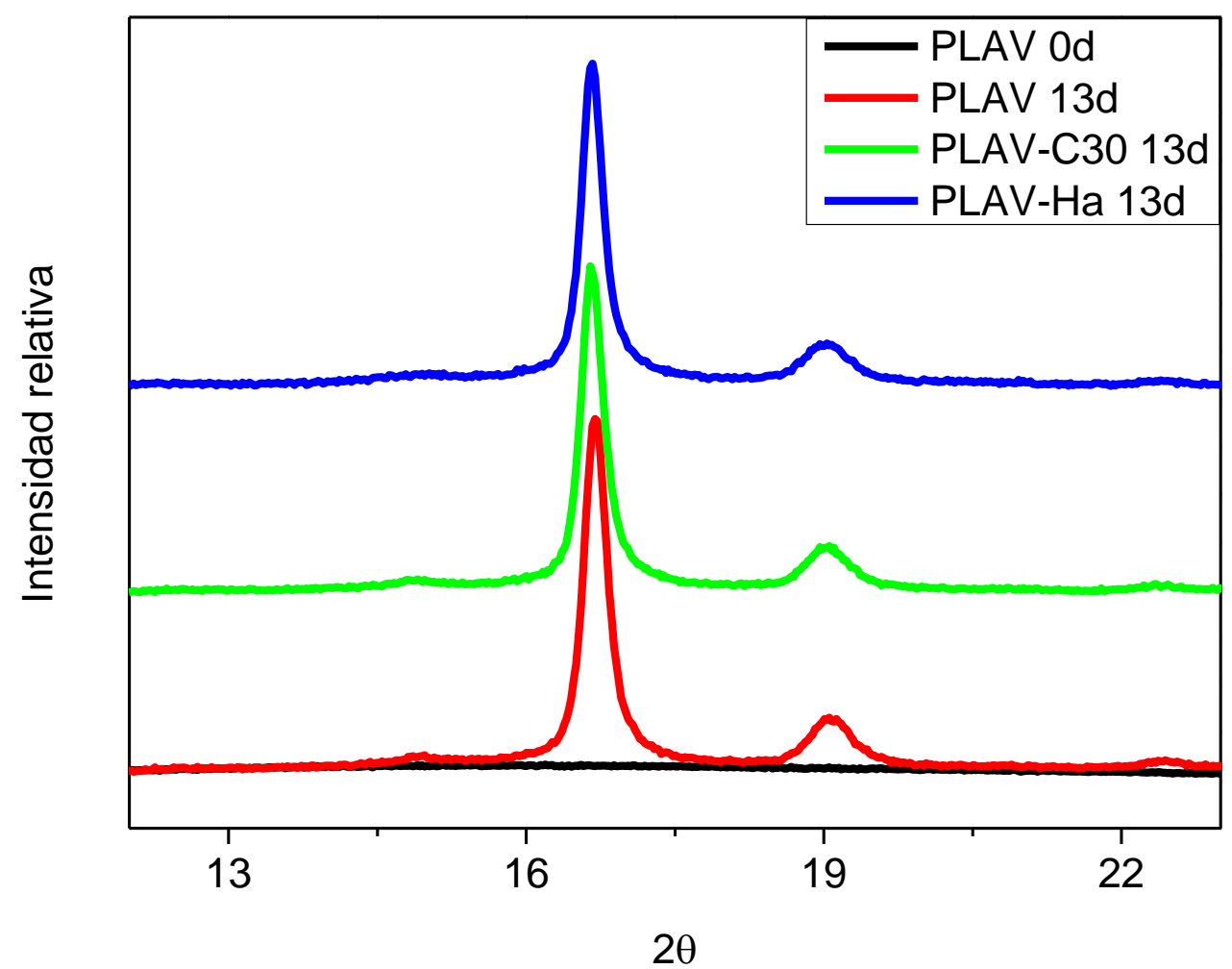

Fig. 44. Difractogramas del PLAV. PLAV-C30 y PLAV-Ha antes y después de 13 días de inmersión a $58^{\circ} \mathrm{C}$.

Si se comparan los valores de cristalinidad obtenidos mediante XRD y FTIR se puede observar que los valores obtenidos mediante XRD son más grandes que aquellos calculados a partir de FTIR, pero que la tendencia seguida en ambos casos es muy similar. Mediante ambos métodos se observa un crecimiento importante de la cristalinidad en los primeros dos días de inmersión, para luego encontrar que la cristalinidad aumenta sólo ligeramente hasta los 13 días de inmersión. Estos resultados indican que la cristalización a $58{ }^{\circ} \mathrm{C}$ es significativamente más rápida que a $37{ }^{\circ} \mathrm{C}$, en la que, como se ha indicado anteriormente, apenas se observó una cristalización naciente después de 84 días en algunos materiales. Esta diferencia se debe a varios factores, entre los cuales se encuentran la mayor velocidad de la degradación hidrolítica a $58{ }^{\circ} \mathrm{C}$ (observada mediante medidas de viscosidad intrínseca), que genera cadenas más cortas y por tanto facilita la cristalización. Sin embargo, a tiempos tan cortos, el principal promotor de la cristalización es el efecto plastificante del agua que, a temperaturas tan próximas a $T_{g}$, aumenta 
significativamente la movilidad de las cadenas poliméricas, facilitando la formación de estructuras cristalinas.

Para poder explicar las diferencias observadas entre los valores calculados mediante XRD y espectroscopía FTIR, hay que tener en cuenta que la degradación hidrolítica es más importante en el interior del material que en la superficie de este, debido al efecto catalítico de los grupos - $\mathrm{COOH}$ formados en las primeras etapas de la degradación hidrolítica. Este efecto catalítico es menor en las capas externas del material, ya que los compuestos carboxílicos pueden ser extraídos con mayor facilidad hacia el medio de inmersión. Es precisamente en estas capas externas, menos degradadas, donde se realizan las medidas mediante FTIR-ATR, obteniendo valores más pequeños que los obtenidos mediante $\mathrm{XRD}$, ya que los rayos $\mathrm{X}$ penetran a una mayor profundidad $\mathrm{y}$ analizan una mayor porción del material.

En la Tabla 13 se puede observar el efecto de las diferentes arcillas en la cristalización del PLA. Por una parte, el PLAV y PLAV-C30 presentan un comportamiento muy parecido. Este resultado podría ser un tanto sorprendente, ya que diversos estudios señalan que la montmorillonita tiene un efecto nucleante en el PLA, promoviendo la cristalización del PLA [86,88]. Sin embargo, la buena dispersión de las láminas de arcilla en el PLA podría también dificultar la movilidad de las cadenas poliméricas, impidiendo la formación de estructuras cristalinas. La presencia de estos dos efectos contrapuestos podría explicar la poca diferencia entre los valores de cristalinidad del PLAV y del PLAV-C30. Por otra parte, se puede apreciar que la presencia de la haloisita disminuye sensiblemente la cristalinidad del PLA. Este comportamiento puede ser debido a varios factores: en primer lugar, al igual que la montmorillonita, la presencia de los nanotubos de haloisita limita la movilidad de las cadenas del PLA, impidiendo la formación de estructuras cristalinas. En segundo lugar, las medidas de viscosidad intrínseca reflejaron que la presencia de haloisita ocasiona una ralentización de la degradación hidrolítica del PLA, dificultando así la formación de estructuras cristalinas.

Además de proveer información sobre el grado de cristalinidad, la espectroscopía FTIR y los ensayos de XRD pueden proveer información acerca de la naturaleza de las estructuras cristalinas formadas durante la inmersión. Por ejemplo, Meaurio et al. reportan que se puede calcular la contribución de las diferentes formas cristalinas ( $\alpha$ y $\left.\alpha^{\prime}\right)$ a la cristalinidad del PLA estudiando la banda $\mathrm{C}=\mathrm{O}\left(\mathrm{a} 1750 \mathrm{~cm}^{-1}\right)$ del PLA [72]. Sin embargo, en nuestro caso esta banda también se ve afectada por los productos de la degradación hidrolítica del PLA, por lo que no puede ser considerada en las condiciones de nuestros experimentos. Sin embargo, otros autores como Cheng et al. [33] han encontrado que la presencia de las diferentes formas cristalinas ocasiona algunos cambios en la banda de absorción ubicada a $920 \mathrm{~cm}^{-1}$. La presencia de la forma $\alpha$ desplaza esta banda hacia números de onda más bajos, ya que el mayor orden de esta forma cristalina fomenta a la existencia de interacciones más fuertes entre las cadenas poliméricas, lo que debilita las vibraciones responsables de esta absorción. 
En la Fig. 45 se presenta la ampliación de la banda de $920 \mathrm{~cm}^{-1}$ de muestras de PLA cristalizadas a diferentes temperaturas con objeto de obtener diferentes morfologías cristalinas: en color negro un PLA cristalizado, en seco, durante 4 horas a $80{ }^{\circ} \mathrm{C}$, obteniéndose la forma $\alpha$; en rojo un PLA cristalizado, en seco, durante 25 minutos a 130 ${ }^{\circ} \mathrm{C}$, obteniéndose la forma $\alpha$; y en azul una muestra de PLAV sumergida durante 13 días a $58{ }^{\circ} \mathrm{C}$ en un tampón de fosfatos. Como se puede observar, el espectro de la muestra sumergida se encuentra entre los de los dos polimorfos puros, lo que indica que en el material sumergido durante 13 días coexisten ambas formas cristalinas.

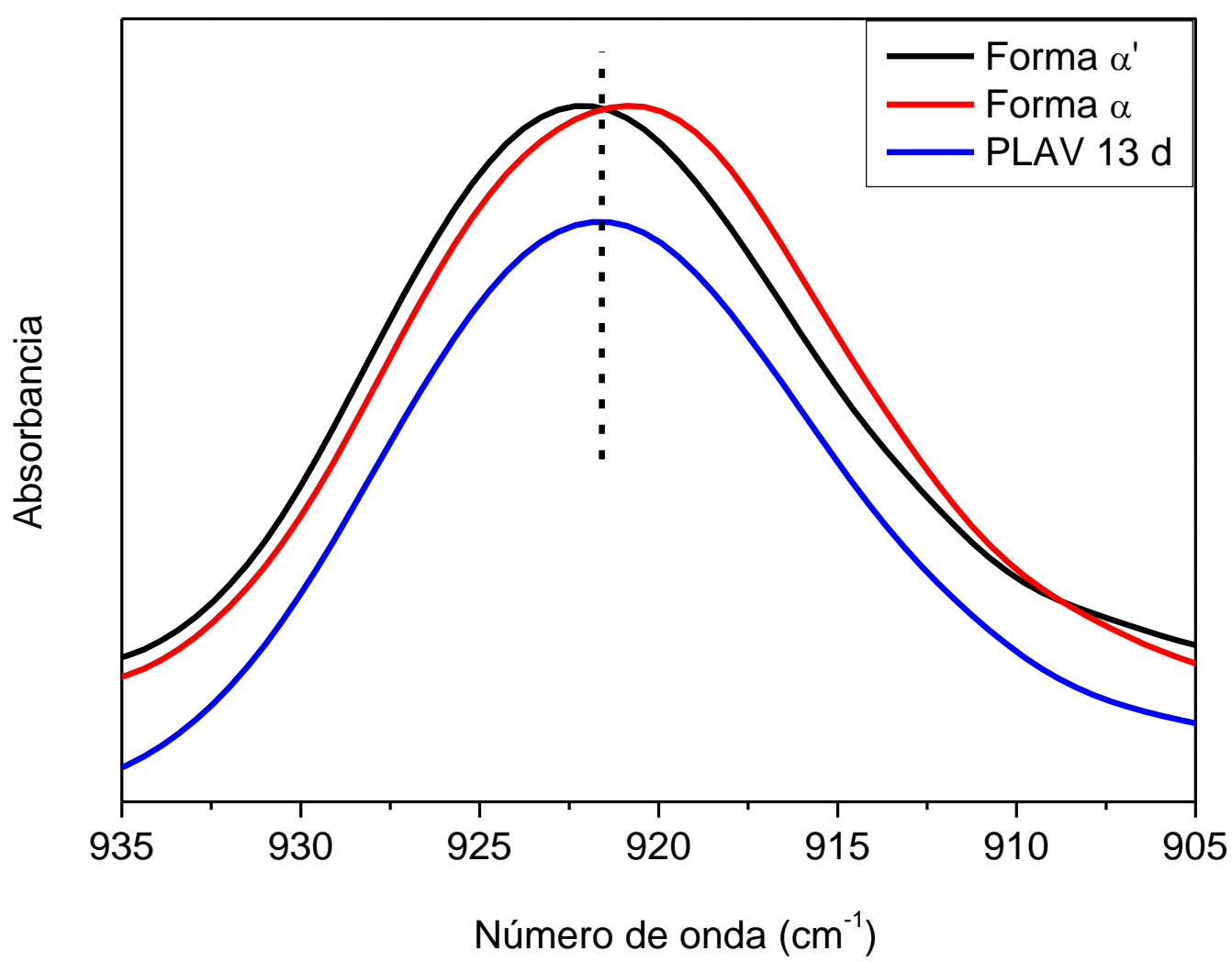

Fig. 45. Banda de absorción de $920 \mathrm{~cm}^{-1}$ del PLA con diferentes formas cristalinas.

Las diferentes formas cristalinas presentes en las muestras sumergidas a $58{ }^{\circ} \mathrm{C}$ fueron también estudiadas mediante XRD. En este caso, las principales diferencias entre las formas cristalinas se encuentran en las reflexiones características de los planos (110), (200) y (203), que se desplazan hacia ángulos más altos en la forma $\alpha$, debido al mayor orden de estas estructuras, tal y como señalan algunos estudios realizados con PLA cristalizado a diferentes temperaturas [33,35,85,118]. La Fig. 46 y la Tabla 14 recogen los resultados obtenidos a partir de los ensayos XRD de las diferentes muestras. El espaciado reticular, $d$, fue calculado utilizando la Ley de Bragg (Ec. 3), y la proporción de fase $\alpha, C_{\alpha}$, fue calculada utilizando la Ec. 5. 
En primer lugar es importante destacar que los valores de $d_{(110) /(200)}$ de todas las muestras se encuentran entre los valores reportados por Pan et al. [85] para las formas $\alpha$ $\left(d_{(110) /(200)}=0,529\right)$ y $\alpha^{\prime}\left(d_{(110) /(200)}=0,536\right)$, lo que indica que en ninguno de los materiales se encuentra presente una sola forma cristalina, sino que se ha formado una mezcla de ambas, tal y como se observa en los espectros FTIR.

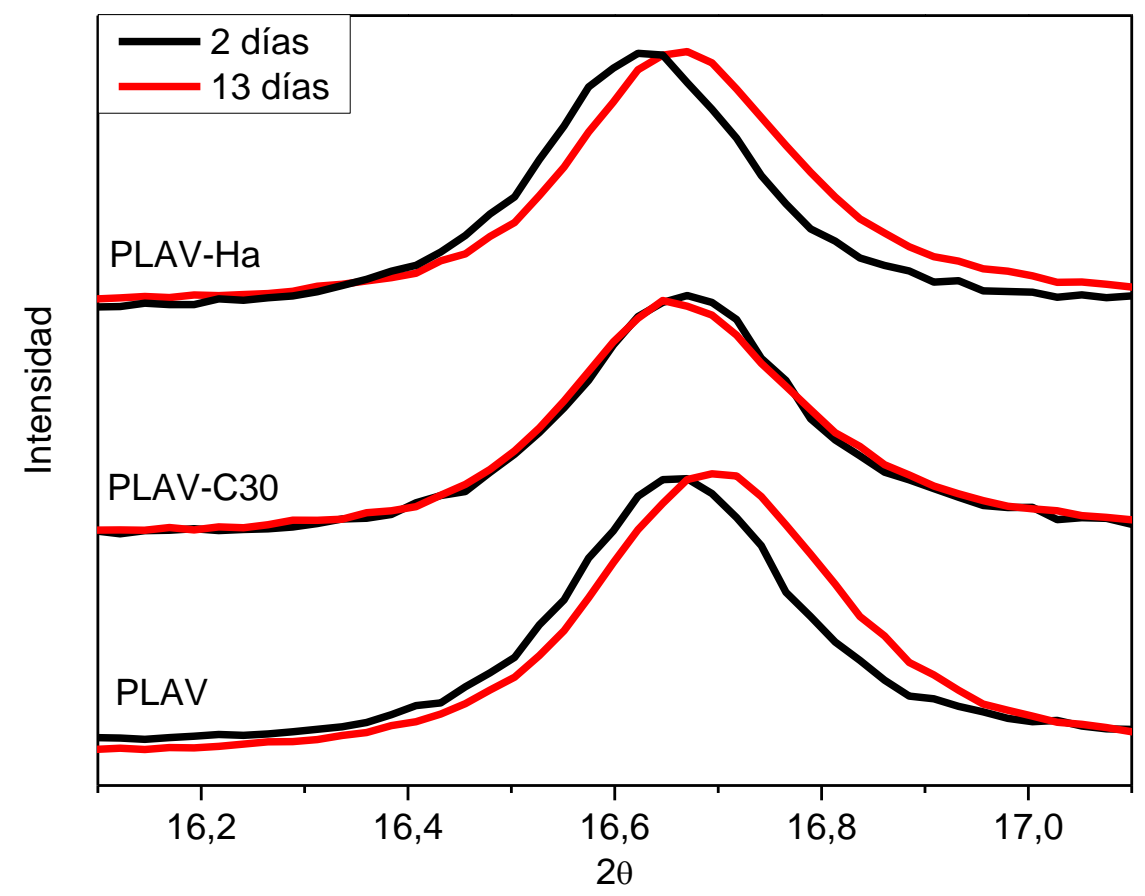

Fig. 46. Picos de reflexión (110)/(200) de los diferentes materiales después de 2 y 13 días de inmersión a $58^{\circ} \mathrm{C}$.

En segundo lugar, la Fig. 46 y la Tabla 14 muestran que tanto el PLAV como el PLAV-Ha presentan un cambio importante en su estructura cristalina a medida que aumenta el tiempo de inmersión. En ambos materiales se observa un desplazamiento del pico de reflexión (110)/(200) hacia ángulos más altos, lo que indica un aumento del espaciado reticular y, por lo tanto, un aumento del contenido de la forma $\alpha$. Este comportamiento puede ser debido a dos factores: por una parte, la formación de cadenas de PLA más cortas, como consecuencia de la degradación hidrolítica, que tendrían mayor movilidad y podrían formar estructuras cristalinas más ordenadas; por otra parte, el efecto plastificante del agua podría facilitar la transformación de los cristales $\alpha$ iniciales en cristales $\alpha$, más organizados. Sin embargo, el comportamiento del PLAV-C30 es diferente, ya que muestra el contenido de fase $\alpha$ más alto después de 2 días de inmersión, pero no parece haber cambios después de 13 días de inmersión. Para explicar estos resultados es necesario tomar en cuenta varios factores: en primer lugar, la arcilla C30 actúa como un catalizador de la degradación hidrolítica y como un agente nucleante, lo que facilitaría la formación de estructuras cristalinas más ordenadas a tiempos cortos; en 
segundo lugar, la presencia de la arcilla podría dificultar la transformación $\alpha^{\prime} \rightarrow \alpha$ a tiempos largos.

Tabla 14. Espacio reticular de la reflexión $(110) /(200)$ y proporción de la forma $\alpha$ de las muestras después de 2 y 13 días de inmersión a $58^{\circ} \mathrm{C}$.

\begin{tabular}{cccc}
\hline Material & Tiempo (días) & $\mathbf{d}_{(\mathbf{1 1 0 / 2 0 0})}(\mathbf{n m})$ & $\mathbf{C \alpha}(\boldsymbol{\%})$ \\
\hline PLAV & 2 & 0,5325 & 50 \\
& 13 & 0,5309 & 73 \\
\hline PLAV-C30 & 2 & 0,5319 & 59 \\
& 13 & 0,5319 & 59 \\
\hline PLAV-Ha & 2 & 0,5331 & 41 \\
& 13 & 0,5315 & 64 \\
\hline
\end{tabular}

En cuanto a los ensayos de DSC, la Fig. 47 y la Tabla 15 recoge los resultados obtenidos para el PLAV y el PLAR sumergido a $58^{\circ} \mathrm{C}$. Se pueden observar, tanto en el PLAV como en el PLAR, algunos cambios importantes a medida que aumenta el tiempo de inmersión. En primer lugar, se puede observar la desaparición progresiva de la transición vítrea y del pico de envejecimiento físico. Esto se debe al aumento de la cristalinidad del PLA, observado mediante FTIR y XRD. La presencia de estructuras cristalinas limita la movilidad de las cadenas poliméricas presentes en las regiones amorfas del material, a las cuales se encuentran asociados los fenómenos de la transición vítrea y el envejecimiento físico. 


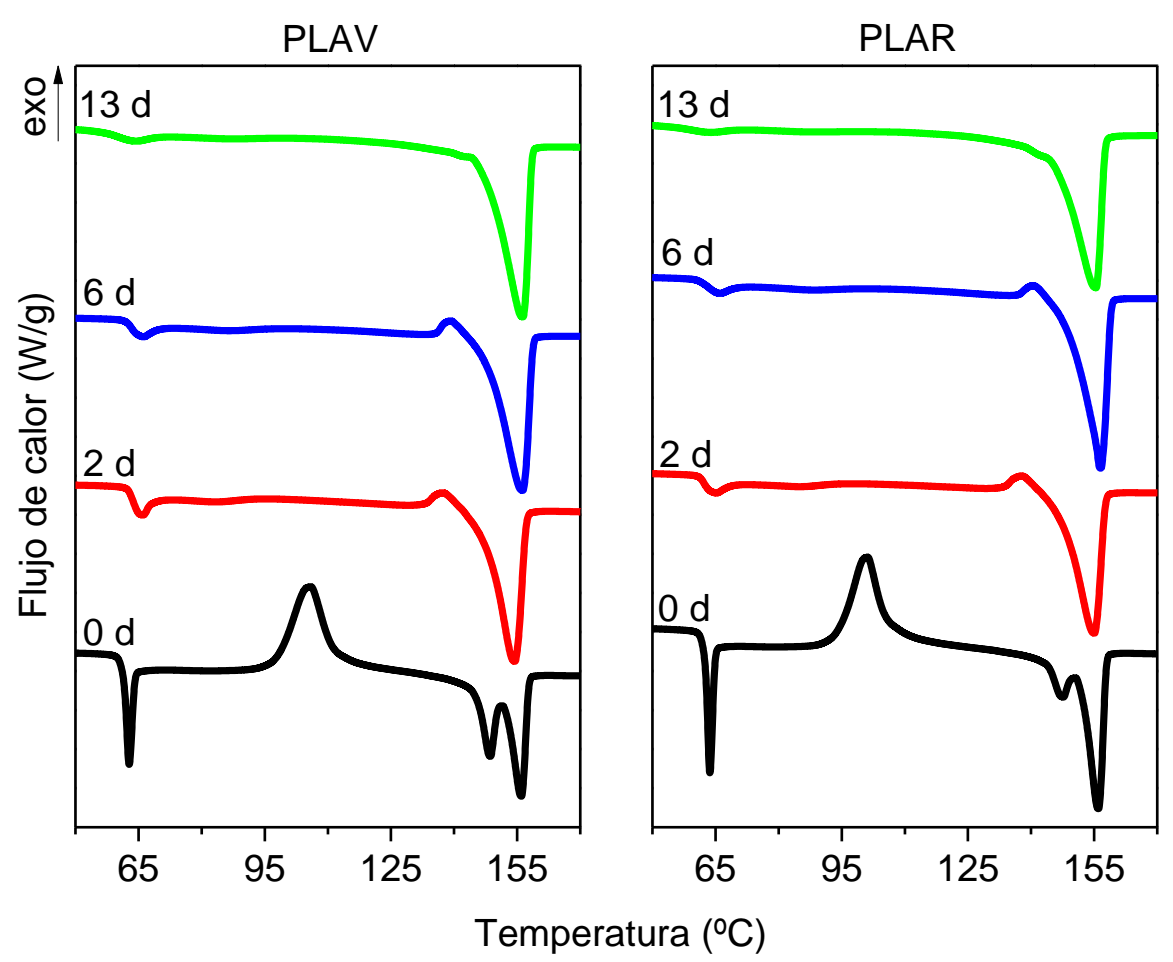

Fig. 47. Primer barrido de calentamiento del PLA, virgen y reciclado, sumergido a 58 ${ }^{\circ} \mathrm{C}$.

En segundo lugar, se puede observar que los valores del pico de cristalización en frío disminuyen de manera significativa después de tan sólo dos días de inmersión. Un comportamiento similar ya ha sido reportado por Badía et al. [13] en su estudio de la degradación hidrotérmica del PLA a temperaturas por encima de $50{ }^{\circ} \mathrm{C}$, y ha sido atribuido a que la mayoría de las regiones cristalizables ya han cristalizado durante la inmersión. Otros autores, como Deroiné et al. [43] sugieren además que los altos valores de cristalinidad limitan la movilidad de las cadenas poliméricas, y por lo tanto estas no son capaces de reordenarse para formar nuevas estructuras cristalinas.

En tercer lugar, la Tabla 15 muestra como aumentan los valores de la entalpía de fusión y de la cristalinidad durante la inmersión, coincidiendo con lo que se ha observado en la espectroscopía FTIR y los difractogramas de rayos X. Sin embargo, si se comparan los valores de cristalinidad de DSC con aquellos obtenidos mediante FTIR y XRD (Tabla 13), se puede observar que los valores obtenidos mediante DSC son bastante más pequeños. De hecho, después de 13 días de inmersión, el PLAV tiene una cristalinidad de $42 \%$ de acuerdo con DSC, $48 \%$ de acuerdo con espectroscopía FTIR y del $60 \%$ de acuerdo con XRD. Esta diferencia se debe a que al calcular la cristalinidad mediante DSC, se toma en cuenta la entalpía de un cristal $\alpha$ perfecto $\left(\Delta \mathrm{H}_{\infty}=93,1 \mathrm{~J} / \mathrm{g}\right)$, pero en realidad existe una mezcla de las formas $\alpha^{\prime}$ y $\alpha$. La entalpía de fusión de esta mezcla debería ser más pequeña, ya que la forma $\alpha$ ' es menos ordenada. Rathi et al. [98] señalan que el valor de $\Delta H_{\infty}$ para un cristal $\alpha$ ' es $60 \mathrm{~J} / \mathrm{g}$. Por lo tanto, los valores reales de cristalinidad deben ser superiores a los determinados por DSC, aunque las tendencias observadas utilizando 
esta técnica deberían ser válidas para el estudio del efecto del reciclado en los cambios estructurales durante la inmersión a $58^{\circ} \mathrm{C}$.

En cuarto lugar, en la Fig. 47 se pueden observar también cambios en el pico exotérmico correspondiente a la transición $\alpha^{\prime} \rightarrow \alpha$. Este pico se encuentra presente después de 2 y 6 días de inmersión, indicando la presencia de cristales $\alpha$ ' en el PLA. Sin embargo, después de 13 días de inmersión este pico desaparece, tanto en el PLAV como en PLAR, a pesar de que los ensayos de espectroscopía FTIR y de XRD muestran que aún existen cristales $\alpha$ ' a los 13 días de inmersión. Este comportamiento podría ser debido a la degradación hidrolítica del PLA, ya que un estudio realizado por Pan et al. [84], utilizando PLA de diferentes pesos moleculares, concluyó que, si el peso molecular es muy bajo, la transición $\alpha^{\prime} \rightarrow \alpha$ no se observa durante el calentamiento. Dicha desaparición podría estar relacionada con la proximidad entre la temperatura de transición de fase y la temperatura de equilibrio, que llevaría a las estructuras cristalinas, formadas por cadenas más cortas, a comenzar a fundirse antes de la transición $\alpha^{\prime} \rightarrow \alpha$.

Finalmente, es importante destacar que el comportamiento, analizado mediante DSC, del PLAV y el PLAR sumergido a $58{ }^{\circ} \mathrm{C}$ es muy similar, y que el mismo comportamiento fue obtenido a partir de ensayos de espectroscopía infrarroja. Este resultado no coincide con lo observado en la cinética de absorción de agua, donde el PLAR parecía tener una mayor cristalinidad, lo que ocasionó un descenso en su coeficiente de difusión. Sin embargo, la estimación del coeficiente de difusión se lleva a cabo considerando tiempos muy cortos de inmersión ( 2 horas) en los que es posible que la diferencia de peso molecular entre el PLAV y el PLAR influyera en la movilidad de las cadenas, y por tanto en la cristalinidad de los materiales.

Tabla 15. Evolución de las entalpías y de la cristalinidad del PLA, virgen y reciclado, sumergido a $58^{\circ} \mathrm{C}$.

\begin{tabular}{cccccc}
\hline Material & $\begin{array}{c}\text { Tiempo } \\
(\mathbf{d} \text { lías })\end{array}$ & $\begin{array}{c}\Delta \mathbf{H}_{\text {crist }} \\
(\mathbf{J} / \mathbf{g})\end{array}$ & $\begin{array}{c}\Delta \mathbf{H}_{\boldsymbol{\alpha}^{\prime} \rightarrow \boldsymbol{\alpha}} \\
(\mathbf{J} / \mathbf{g})\end{array}$ & $\begin{array}{c}\Delta \mathbf{H}_{\mathbf{f u s}} \\
(\mathbf{J} / \mathbf{g})\end{array}$ & $\begin{array}{c}\mathbf{X}_{\mathbf{C}} \\
(\boldsymbol{\%})\end{array}$ \\
\hline \multirow{4}{*}{ PLAV } & 0 & 27,1 & 0 & 27,6 & 1 \\
& 2 & 3,0 & 1,8 & 32,1 & 29 \\
& 6 & 2,5 & 1,9 & 33,6 & 31 \\
& 13 & 0 & 0,0 & 39,5 & 42 \\
\hline \multirow{3}{*}{ PLAR } & 0 & 27,6 & 0 & 28,3 & 1 \\
& 2 & 2,7 & 1,9 & 30,5 & 30 \\
& 6 & 2,5 & 1,5 & 33,0 & 33 \\
& 13 & 0 & 0 & 40,3 & 43 \\
\hline
\end{tabular}

En cuanto al comportamiento de los nanocomposites PLA-C30, la Fig. 48 y la Tabla 16 muestran que el comportamiento seguido por este material es muy similar al del PLA puro. Se puede observar la desaparición de la transición vítrea (y del pico de 
envejecimiento físico), un descenso considerable de la entalpía de cristalización en frío y un aumento significativo de la cristalinidad. Estos resultados coinciden, en parte, con los de XRD y FTIR, en los que se observa que el PLAV y el PLAV-C30 presentan grados de cristalinidad similares después de 2 y 13 días de inmersión. Sin embargo, los ensayos de DSC no permiten observar la mayor proporción de fase $\alpha$ ' presente en el nanocomposite.

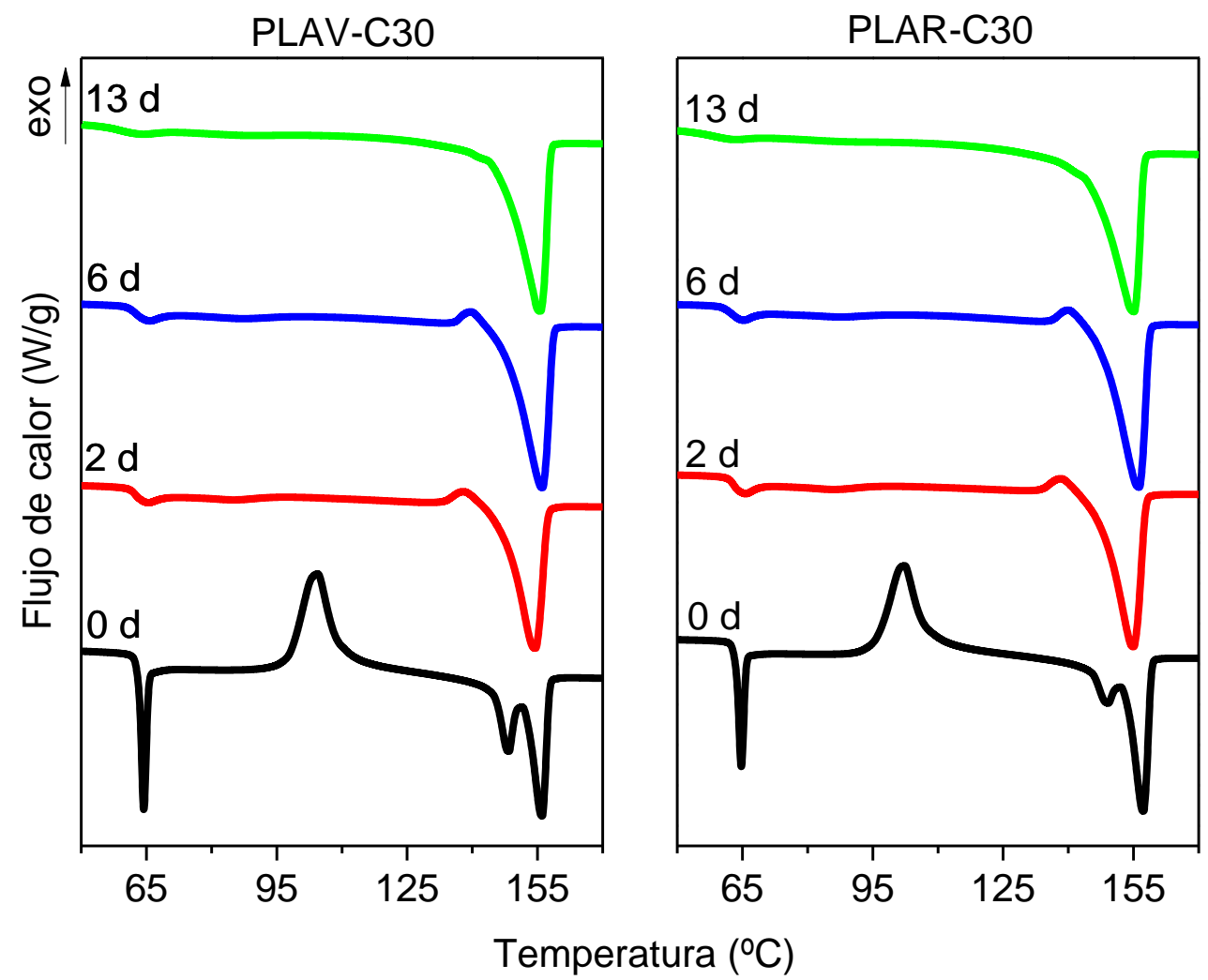

Fig. 48. Primer barrido de calentamiento del nanocomposite PLA-C30, virgen y reciclado, sumergido a $58^{\circ} \mathrm{C}$.

En cuanto al efecto del reciclado en los nanocomposites, en la Tabla 16 solo se puede observar una cristalinidad ligeramente mayor en el PLAR-C30 después de 2 días de inmersión, luego, conforme aumenta el tiempo los valores de cristalinidad del nanocomposite virgen y reciclado van acercándose progresivamente. Para explicar este comportamiento hay que considerar la mejora de la dispersión de la arcilla durante el reciclado. Dicha mejora podría favorecer el efecto nucleante de la montmorillonita, promoviendo la cristalización del PLA a tiempos relativamente cortos. Sin embargo, a tiempos más largos, el efecto de la severa degradación del PLA parece prevalecer, ya que las cadenas cortas presentes en ambas muestras cristalizan con mayor facilidad conforme aumenta el tiempo de inmersión. Resultados similares fueron obtenidos mediante FTIR, en donde no se observaron diferencias importantes entre el PLAV-C30 y el PLAR-C30. 
Tabla 16. Evolución de las entalpías y de la cristalinidad del nanocomposite PLA-C30, virgen y reciclado, sumergido a $58^{\circ} \mathrm{C}$.

\begin{tabular}{cccccc}
\hline Material & $\begin{array}{c}\text { Tiempo } \\
(\mathbf{d} \text { lías })\end{array}$ & $\begin{array}{c}\Delta \mathbf{H}_{\text {crist }} \\
(\mathbf{J} / \mathbf{g})\end{array}$ & $\begin{array}{c}\Delta \mathbf{H}_{\boldsymbol{a}^{\prime} \rightarrow \boldsymbol{\alpha}} \\
(\mathbf{J} / \mathbf{g})\end{array}$ & $\begin{array}{c}\Delta \mathbf{H}_{\text {fus }} \\
(\mathbf{J} / \mathbf{g})\end{array}$ & $\begin{array}{c}\mathbf{X}_{\mathbf{C}} \\
(\boldsymbol{\%})\end{array}$ \\
\hline \multirow{3}{*}{ PLAV-C30 } & 0 & 26,8 & 0 & 27,8 & 1 \\
& 2 & 2,5 & 1,7 & 29,0 & 27 \\
& 6 & 2,9 & 1,6 & 33,4 & 32 \\
& 13 & 0 & 0 & 39,0 & 42 \\
\hline \multirow{3}{*}{ PLAR-C30 } & 0 & 26,0 & 0 & 27,2 & 1 \\
& 2 & 2,5 & 1,7 & 31,8 & 32 \\
& 6 & 2,9 & 1,3 & 34,7 & 35 \\
& 13 & 0 & 0 & 38,9 & 43 \\
\hline
\end{tabular}

Si bien el PLA puro y el nanocomposite con C30 presentan comportamientos similares, la Fig. 49 y la Tabla 17 muestran que el nanocomposite PLA-Ha presenta algunas diferencias. Por una parte, se puede observar que estos nanocomposites con haloisita presentan el pico exotérmico correspondiente a la transición $\alpha^{\prime} \rightarrow \alpha$, incluso después de 13 días de inmersión. Este comportamiento podría ser debido a que, tal y como se observa en las medidas de viscosidad intrínseca, los nanocomposites PLA-Ha presentan una mayor viscosidad que el PLA y el PLA-C30 después de 13 días de inmersión. La presencia de cadenas más largas, y con menor movilidad, hace más difícil la formación de estructuras cristalinas más ordenadas, como la forma $\alpha$. Así mismo, la presencia de los nanotubos parece disminuir la movilidad de las cadenas de PLA, promoviendo así la formación de estructuras cristalinas menos ordenadas.

Por otra parte, la Tabla 17 muestra que los valores de cristalinidad del nanocomposite PLA-Ha son más bajos que los observados previamente para el PLA puro y el nanocomposite PLA-C30. Este comportamiento coincide con el observado mediante espectroscopía FTIR y XRD, y se debe posiblemente también a la menor degradación del nanocomposite PLA-Ha y a la disminución de la movilidad de las cadenas de PLA debido a la presencia de los nanotubos de haloisita.

Finalmente, se debe resaltar que el efecto del reciclado en los cambios estructurales de los nanocomposites PLA-Ha es pequeño, tal y como sucede con el PLA puro y el PLA-C30, ya que apenas se aprecian diferencias en los valores de cristalinidad. 


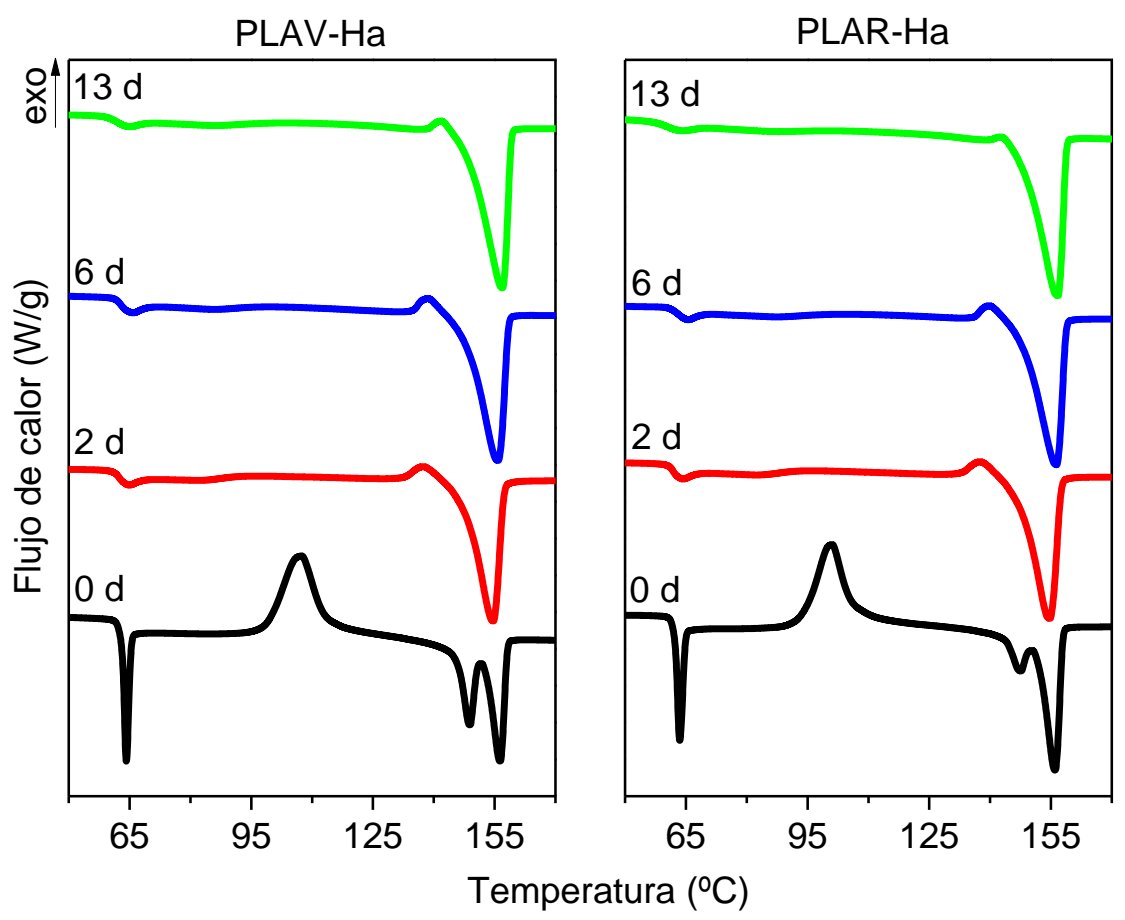

Fig. 49. Primer barrido de calentamiento del nanocomposite PLA-Ha, virgen y reciclado, sumergido a $58^{\circ} \mathrm{C}$.

En resumen, a $37^{\circ} \mathrm{C}$ todos los materiales presentan un descenso de la temperatura de cristalización en frío, un aumento de la cristalinidad y la desaparición del pico de fusión de menor temperatura. Todos estos cambios son consecuencia, en gran parte, de la degradación hidrolítica, ya que las cadenas más cortas generadas durante este proceso pueden moverse con mayor facilidad, favoreciendo la formación de estructuras cristalinas. Sin embargo, tanto en el PLA puro como en el nanocomposite PLA-Ha los materiales reciclados muestran una cristalinidad ligeramente mayor después de 84 días de inmersión, mientras que en el nanocompuesto con C30 no hay diferencias significativas. En cualquier caso, el efecto del reciclado es pequeño y solamente apreciable después de tiempos largos de inmersión, por lo que el potencial uso de materiales basados en PLA reciclado en aplicaciones de envasado de alimentos no puede descartarse, al menos desde un punto de vista de cambios estructurales como consecuencia de la degradación hidrolítica. A $58^{\circ} \mathrm{C}$, los cambios estructurales son más importantes, quedando demostrado mediante espectroscopía FTIR, XRD y DSC la formación de diferentes formas cristalinas en el PLA durante la inmersión, como consecuencia de la degradación hidrolítica. El reciclado parece no tener una influencia importante sobre dichos cambios estructurales, ya que apenas se aprecian diferencias entre las muestras vírgenes y recicladas. 
Tabla 17. Evolución de las entalpías y de la cristalinidad del nanocomposite PLA-Ha, virgen y reciclado, sumergido a $58^{\circ} \mathrm{C}$.

\begin{tabular}{|c|c|c|c|c|c|}
\hline Material & $\begin{array}{c}\text { Tiempo } \\
\text { (días) }\end{array}$ & $\begin{array}{c}\Delta \mathbf{H}_{\text {crist }} \\
(\mathrm{J} / \mathrm{g})\end{array}$ & $\begin{array}{c}\Delta \mathbf{H}_{\alpha^{\prime} \rightarrow \alpha} \\
(\mathrm{J} / \mathrm{g})\end{array}$ & $\begin{array}{c}\Delta \mathbf{H}_{\text {fus }} \\
(\mathbf{J} / \mathbf{g})\end{array}$ & $\begin{array}{c}\mathbf{X}_{\mathbf{C}} \\
(\%)\end{array}$ \\
\hline \multirow{4}{*}{ PLAV-Ha } & 0 & 27,2 & 0 & 28,4 & 1 \\
\hline & 2 & 3,0 & 1.9 & 26,4 & 24 \\
\hline & 6 & 2,7 & 1,8 & 34,0 & 32 \\
\hline & 13 & 4,2 & 1,0 & 35,2 & 33 \\
\hline \multirow{4}{*}{ PLAR-Ha } & 0 & 27,4 & 0 & 28 & 1 \\
\hline & 2 & 3 & 2,2 & 32,1 & 29 \\
\hline & 6 & 2,7 & 1,7 & 33,3 & 32 \\
\hline & 13 & 3,8 & 0,4 & 34,3 & 33 \\
\hline
\end{tabular}

\subsection{Evolución de la estabilidad térmica durante la inmersión de los materiales reciclados}

El descenso del peso molecular del PLA, en conjunto con los cambios estructurales observados previamente, podrían afectar la estabilidad térmica del PLA y sus nanocomposites durante la inmersión a diferentes temperaturas. El estudio de la estabilidad térmica es importante para analizar la viabilidad del reprocesado mecánico, ya que, si la estabilidad térmica se redujera de forma importante durante la inmersión, el material sufriría una degradación excesiva durante un eventual reprocesado por extrusión. Para estudiar este fenómeno, se han realizado ensayos de termogravimetría a los materiales a diferentes tiempos de inmersión. Los resultados para ambas temperaturas de inmersión se encuentran recogidos en la Fig. 50 y en la Tabla 18.

En cuanto al comportamiento de los materiales sumergidos a $37^{\circ} \mathrm{C}$, la Fig. 50 muestra que, en todos los casos, después de 84 días de inmersión hay un leve descenso de los valores de $T_{10}$. Este comportamiento se debe a la degradación hidrolítica del PLA durante la inmersión, ya que las cadenas más cortas tienen una menor estabilidad térmica. Cabe destacar que, si bien todos los materiales presentan un descenso similar de $T_{10}$ después de 84 días de inmersión, la evolución de los nanocomposites difiere ligeramente de la del PLA puro. Mientras que en el PLA sin reforzar se puede observar un descenso de $T_{10}$ después de sólo 13 días de inmersión, en los nanocomposites se observa como el valor de $T_{10}$ no varía demasiado durante los primeros 56 días, presentando un descenso después de transcurridos 84 días. En cualquier caso, las diferencias observadas son mínimas.

En cuanto al efecto del reciclado, todas las muestras parecen exhibir un comportamiento similar. Sin embargo, el proceso de lavado parece afectar la evolución de la estabilidad térmica del nanocomposite PLA-C30, debido probablemente a los relativamente bajos valores de peso molecular que presenta este material, incluso antes de la degradación hidrolítica. En cualquier caso, como ya se ha mencionado, las 
diferencias son bastantes pequeñas, coincidiendo con los resultados de viscosidad intrínseca, DSC, FTIR y XRD.
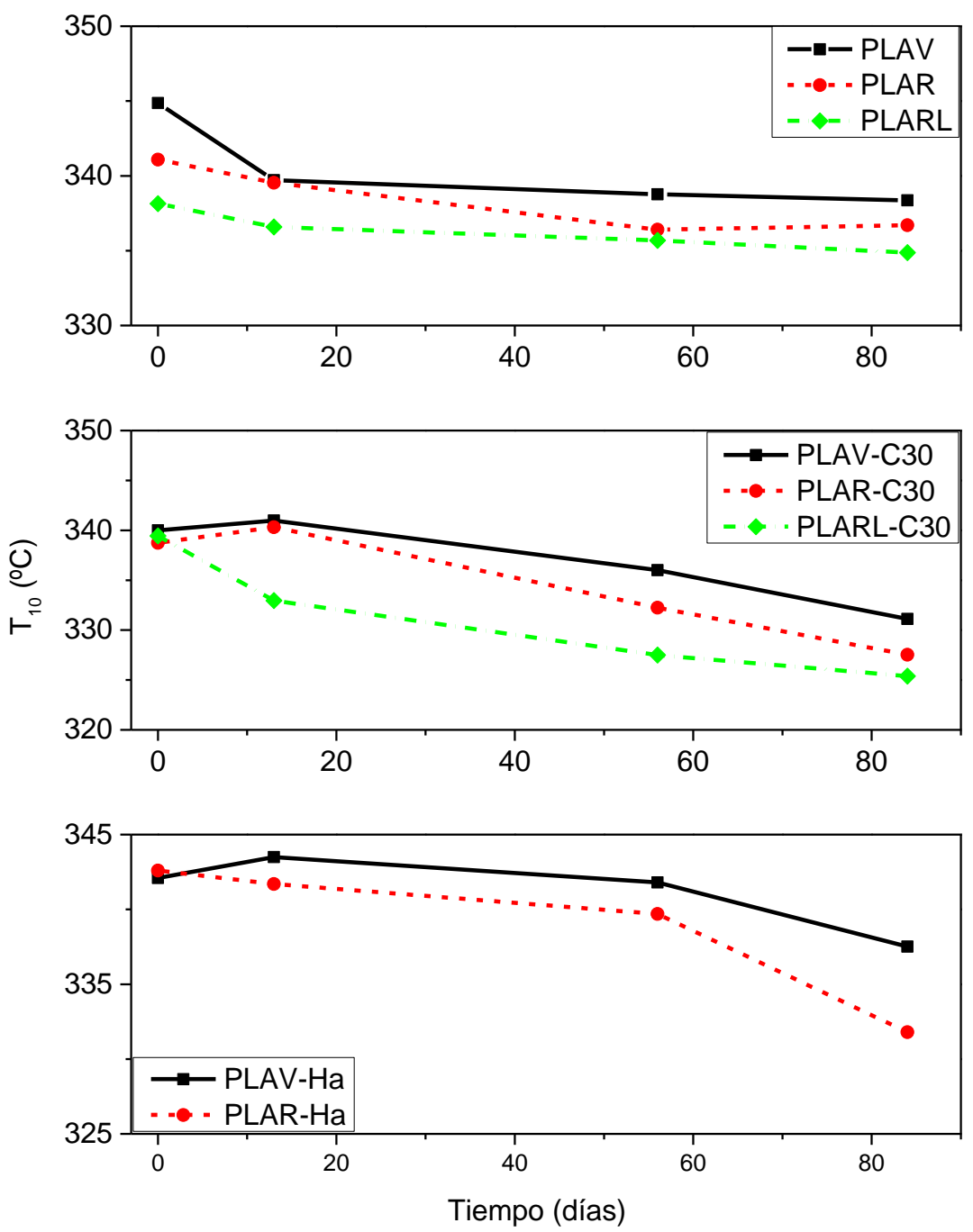

Fig. 50. Evolución de la $\mathrm{T}_{10}$ de los materiales sumergidos a $37^{\circ} \mathrm{C}$

En lo concerniente al comportamiento de las muestras sumergidas a $58{ }^{\circ} \mathrm{C}$, la Tabla 18 muestra los valores de $T_{10}$ de diferentes materiales antes y después de 13 días de inmersión a $58{ }^{\circ} \mathrm{C}$. Se puede observar que, de forma similar a lo ocurrido con la viscosidad intrínseca, después de 13 días de inmersión los valores de $T_{10}$ se reducen de forma significativamente mayor que a $37{ }^{\circ} \mathrm{C}$. Este resultado se debe a la severa degradación sufrida por el PLA en estas condiciones de inmersión. En cuanto al efecto del reciclado, todos los materiales reciclados parecen sufrir descensos de $T_{10}$ ligeramente mayores que sus contrapartes vírgenes, a diferencia de lo observado en las medidas de viscosidad intrínseca, donde el reciclado no tenía un efecto importante. En cualquier caso, las diferencias son muy pequeñas (del orden del 1\%), por lo que el reciclado no parece tener un efecto importante en la evolución de la estabilidad térmica de los materiales sumergidos a $58^{\circ} \mathrm{C}$. 
Tabla 18. Evolución de $\mathrm{T}_{10}$ de los materiales sumergidos a $58^{\circ} \mathrm{C}$.

\begin{tabular}{ccc}
\hline Material & Tiempo (días) & $\mathbf{T}_{\mathbf{1 0}}\left({ }^{\mathbf{0}} \mathbf{C}\right)$ \\
\hline \multirow{2}{*}{ PLAV } & 0 & 344,9 \\
\multirow{2}{*}{ PLAR } & 13 & 323,4 \\
& 0 & 341,1 \\
\multirow{2}{*}{ PLAV-C30 } & 13 & 315,2 \\
\hline \multirow{2}{*}{ PLAR-C30 } & 0 & 340,0 \\
& 13 & 317,1 \\
\multirow{2}{*}{ PLAV-Ha } & 0 & 338,7 \\
& 13 & 311,6 \\
\hline \multirow{2}{*}{ PLAR-Ha } & 0 & 342,1 \\
& 13 & 332,4 \\
& 0 & 342,6 \\
\hline
\end{tabular}

En resumen, los resultados señalan que la degradación observada mediante medidas de viscosidad intrínseca se traduce en un descenso de la estabilidad térmica del PLA y sus nanocomposites. Este descenso es pequeño a $37^{\circ} \mathrm{C}$ y moderado a $58^{\circ} \mathrm{C}$, pero esta última es una temperatura que no se alcanzará frecuentemente en el uso cotidiano del PLA. En cuanto al efecto del reciclado, este parece ser bastante pequeño, incluso en las muestras sometidas a una etapa de lavado, por lo que la estabilidad térmica durante la degradación hidrolítica a temperaturas moderadas no debería ser un factor limitante en la utilización de PLA reciclado.

\subsection{Evolución de la microdureza durante la inmersión de los materiales reciclados}

Como se ha comentado anteriormente, los cambios en la viscosidad intrínseca y en la estructura del PLA durante la inmersión pueden afectar diferentes propiedades de los materiales estudiados, entre las cuales se encuentra la microdureza. En la Fig. 51 se recogen los valores de microdureza de las muestras sumergidas a $37{ }^{\circ} \mathrm{C}$. Se puede observar que en el caso del PLA solo, después de 84 días de inmersión la dureza aumenta un 5, 16 y $13 \%$ para el PLAV, PLAR y PLARL, respectivamente. Este comportamiento podría resultar llamativo, ya que la disminución del peso molecular del PLA, como consecuencia de la degradación hidrolítica, debería afectar negativamente las propiedades mecánicas del material. Sin embargo, la dureza es afectada por otros factores además del peso molecular, como la cristalinidad y la configuración molecular [90]. De hecho, se ha visto en los resultados de DSC que la presencia de agua promueve la relajación estructural (o envejecimiento físico) y esto podría explicar el aumento de la dureza durante la inmersión. Además, se puede observar que el aumento de la dureza es mayor en los materiales reciclados que en el virgen, lo cual podría ser debido a dos factores: por una 
parte, las cadenas más cortas presentes en los materiales reciclados tienen mayor movilidad, lo que las hace más susceptibles al fenómeno del envejecimiento físico; por otra parte, los resultados de DSC y XRD indican una cristalización incipiente en los materiales reciclados, que también podría contribuir al incremento de la dureza de estos materiales.

En cuanto al comportamiento de los nanocomposites, se puede apreciar en la Fig. 51 que, en líneas generales, muestran el mismo comportamiento que el PLA puro, con valores de microdureza crecientes durante la inmersión.
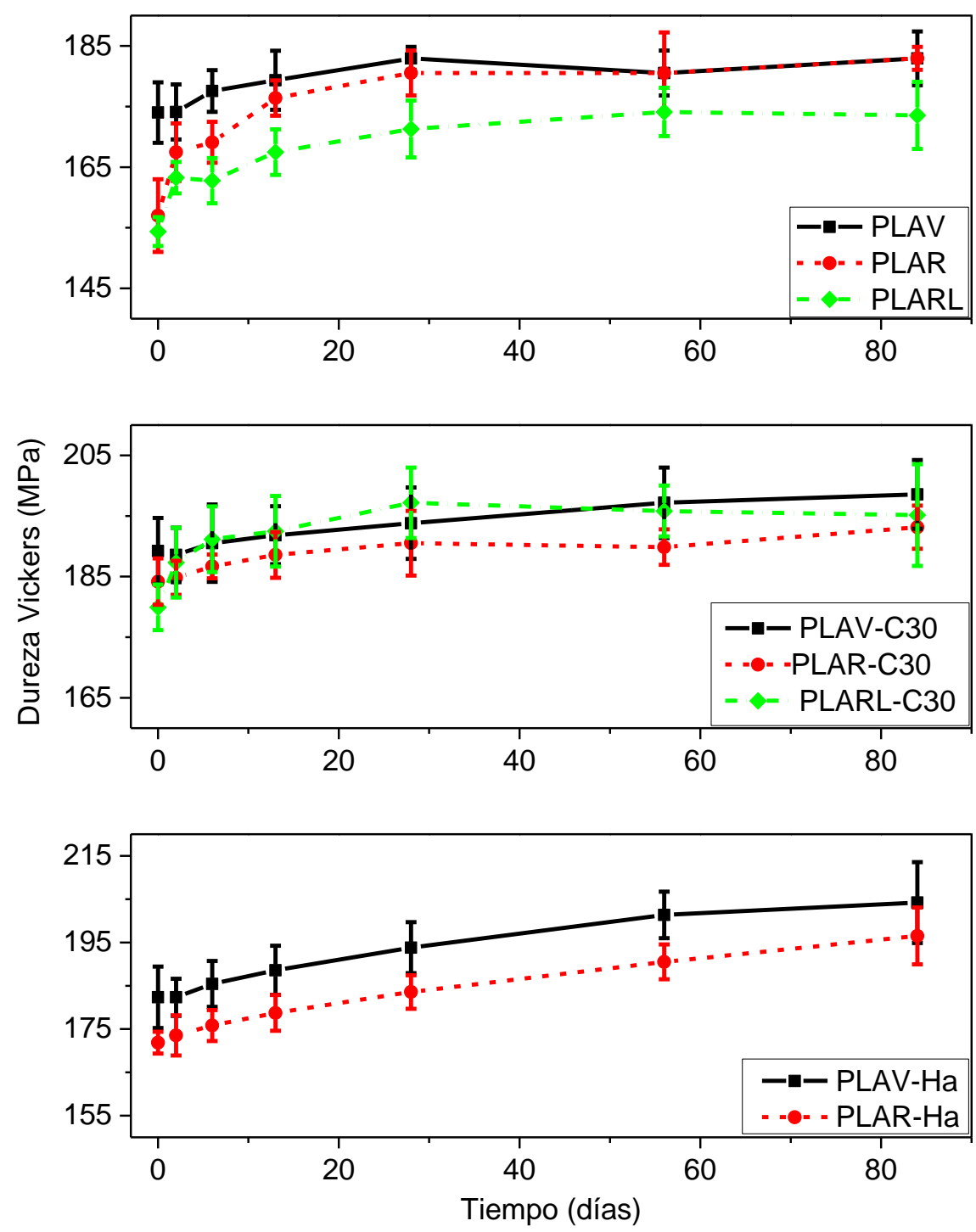

Fig. 51. Evolución de la microdureza de los materiales sumergidos a $37^{\circ} \mathrm{C}$.

En la Tabla 19 se recogen los valores de microdureza de los materiales antes y después de ser sumergidos a $58^{\circ} \mathrm{C}$. En el caso del PLA puro, tanto virgen como reciclado, se puede observar que después de 13 días de inmersión la dureza ha aumentado más de un $25 \%$. Está diferencia tan pronunciada con respecto a lo ocurrido a $37^{\circ} \mathrm{C}$ se debe a los altos valores de cristalinidad observados mediante XRD, espectroscopía FTIR y DSC. En 
cuanto al comportamiento de los nanocomposites, se puede observar que tanto el PLAVC30 como el PLAR-C30 presentan aumentos de la dureza más pequeños, alrededor del $12 \%$, en comparación con el PLA puro, a pesar de que los ensayos de XRD, DSC y espectroscopía muestran que el nanocomposite PLA-C30 presenta valores de cristalinidad similares a los del PLA. Hay que tener en cuenta que los valores iniciales son más altos en los nanocomposites y que los valores finales son bastante similares en todos los materiales, lo que podría explicarse si se supone que la dureza final se debe a la cristalización del polímero. Además, el menor aumento relativo de la dureza en los nanocomposites con C30 podría estar relacionado con las formas cristalinas presentes en los materiales después de 13 días de inmersión. Mientras que en el PLA puro hay un mayor contenido de fase $\alpha$ (con mejores propiedades mecánicas), en el nanocomposite PLA-C30 se observó un mayor contenido de cristales $\alpha$, lo que daría como resultado materiales con menor dureza.

Los nanocomposites PLA-Ha virgen y reciclado también muestran un menor incremento relativo de la dureza que el PLA. En este caso, la diferencia puede deberse también a los menores grados de cristalinidad observados en los nanocomposites PLAhaloisita. Finalmente es importante destacar que el efecto del reciclado en el PLA puro y en ambos nanocomposites parece ser muy pequeño a $58^{\circ} \mathrm{C}$, debido probablemente a que es más importante el efecto de la severa degradación del PLA.

Tabla 19. Evolución de la microdureza de los diferentes materiales sumergidos a $58^{\circ} \mathrm{C}$.

\begin{tabular}{ccc}
\hline Material & Tiempo (días) & Dureza Vickers (MPa) \\
\hline \multirow{3}{*}{ PLAV } & 0 & $174 \pm 5$ \\
& 6 & $197 \pm 8$ \\
PLAR & 13 & $218 \pm 4$ \\
& 0 & $157 \pm 6$ \\
PLAV-C30 & 6 & $161 \pm 2$ \\
& 13 & $201 \pm 3$ \\
\hline PLAR-C30 & 0 & $189 \pm 5$ \\
& 6 & $205 \pm 8$ \\
& 13 & $211 \pm 4$ \\
PLAV-Ha & 0 & $184 \pm 4$ \\
& 6 & $197 \pm 7$ \\
& 13 & $206 \pm 7$ \\
\hline PLAR-Ha & 0 & $182 \pm 7$ \\
& 6 & $200 \pm 5$ \\
& 13 & $212 \pm 5$ \\
& 6 & $172 \pm 3$ \\
& 13 & $191 \pm 7$ \\
\hline
\end{tabular}


Capítulo 5. Efecto del reciclado en la degradación hidrolítica

En resumen, se puede observar que tanto a 37 como a $58{ }^{\circ} \mathrm{C}$ la dureza de los materiales aumenta conforme aumenta el tiempo de inmersión, debido a los cambios estructurales que tienen lugar en el PLA. 


\section{MEJORA DE PROPIEDADES DEL PLA RECICLADO}

Como se ha visto en secciones anteriores, el reciclado mecánico puede causar la degradación del PLA, sobre todo al introducir una etapa de lavado enérgico. Esta degradación tiene un efecto negativo sobre las propiedades mecánicas, térmicas y de barrera del material reciclado, lo que podría comprometer su uso en aplicaciones demandantes, como el envasado, y poner en riesgo el bajo impacto medioambiental de este polímero. Por ello, sería muy interesante la puesta a punto de métodos sencillos y económicos para la mejora de propiedades, la regradación, del plástico de postconsumo reciclado. En esta sección se analizan dos enfoques diferentes para la obtención de forma económica de PLA reciclado con mejores propiedades: la extrusión reactiva y la utilización de arcillas.

\subsection{Mejora de prestaciones de PLA reciclado mediante extrusión reactiva}

La extrusión reactiva consiste en la utilización de diferentes aditivos durante el proceso de extrusión de los plásticos, con el objetivo de modificar las propiedades del material extruido. En este trabajo la extrusión reactiva se utiliza para aumentar el peso molecular promedio del PLA reciclado mecánicamente, y mejorar así sus prestaciones. Para ello se emplean dos reactivos: un extensor de cadena comercial (CESA-Extend) y el peróxido de dicumilo (DCP). En las secciones siguientes se mostrarán los resultados obtenidos, incluyendo el efecto de los diferentes aditivos en la estructura y propiedades del PLA reciclado.

\subsubsection{Viscosidad intrínseca del PLA reciclado mejorado mediante extrusión reactiva}

Los resultados presentados en el capítulo 4 muestran que el PLA sufre un proceso de degradación durante su reciclado mecánico, que depende de las condiciones de uso y reciclado. Por ejemplo, la degradación es más importante si se añade una etapa de lavado enérgico. La presencia de cadenas más cortas se traduce en un descenso de la viscosidad intrínseca, un parámetro muy importante en el procesado de polímeros. A nivel industrial, 
los equipos utilizados para el procesado en fundido (extrusoras, inyectoras, etc.) se operan en condiciones muy específicas de temperaturas, tiempos y esfuerzos, condiciones optimizadas para una determinada viscosidad en el caso de polímeros como PLA. Por lo tanto, una variación importante de la viscosidad intrínseca del polímero podría cambiar el comportamiento del material durante el procesado, afectando negativamente la calidad de los productos obtenidos a partir del material reciclado. Esto dificultaría la obtención de ganancias a partir del PLA reciclado, y, por tanto, comprometería la reciclabilidad del material.

El uso de determinados aditivos en el momento de extruir el material de postconsumo podría redundar en una mejora de la viscosidad intrínseca del PLA. Como se ha comentado en el Capítulo 3, en este trabajo se han empleado 2 aditivos, peróxido de dicumilo (DCP) y un extensor de cadena comercial denominado CESA-Extend. Ambos compuestos promueven las reacciones entre las cadenas de PLA reciclado, permitiendo la unión de varias cadenas y aumentando el peso molecular. Para cuantificar el efecto de estos aditivos en el PLARLH, se midió la viscosidad intrínseca de todos los materiales, y los resultados obtenidos se muestran en la Fig. 52.

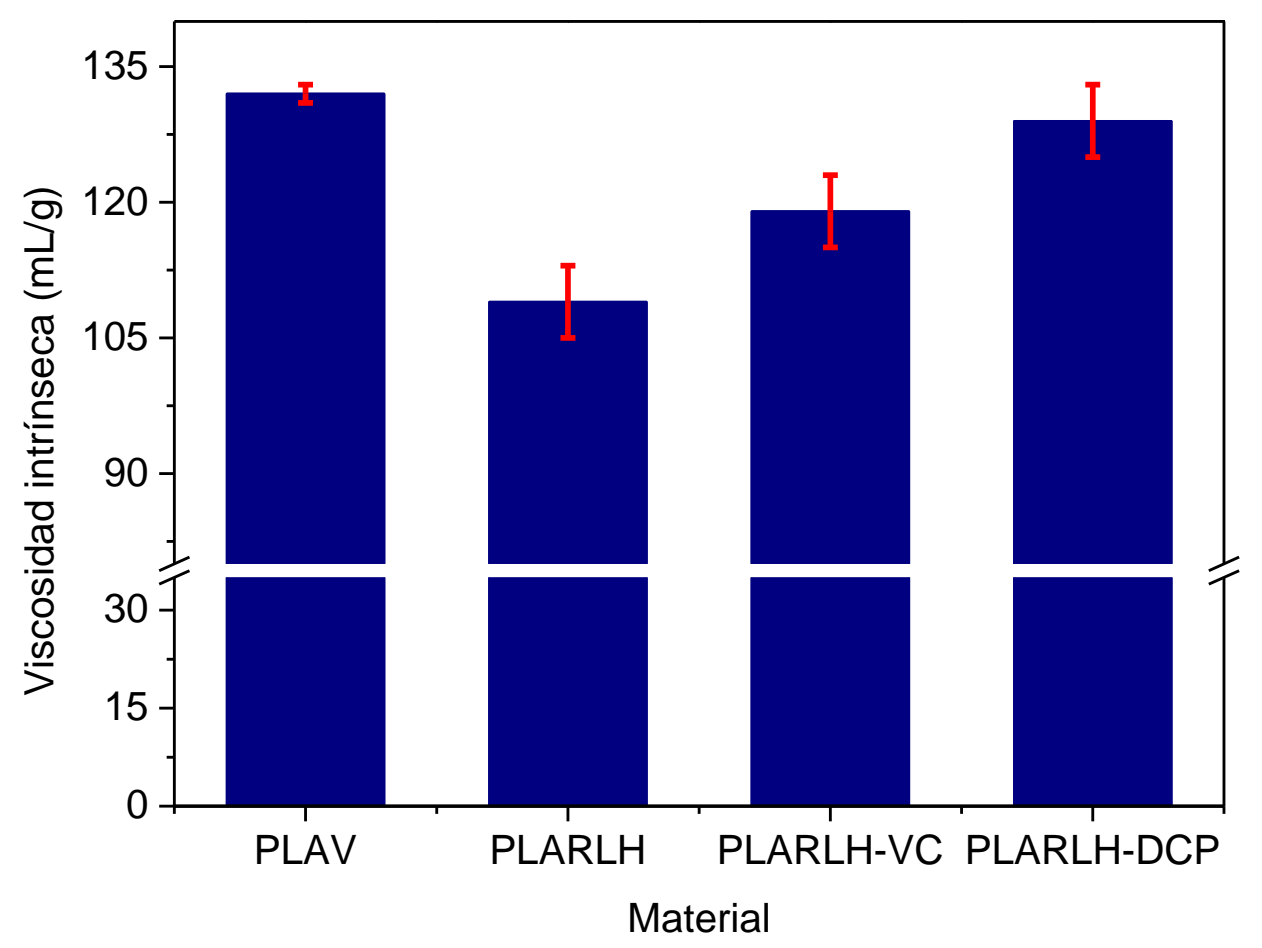

Fig. 52. Viscosidad intrínseca del PLA reciclado extruido mejorado mediante extrusión reactiva.

En la Fig. 52 se puede apreciar que tanto la adición del peróxido de dicumilo (PLARLH-DCP), como la mezcla de PLA reciclado y PLA virgen (50/50) con 1,5\% de extensor de cadena (PLARLH-VC), producen un aumento de la viscosidad intrínseca del material reciclado, especialmente en el material con DCP. Esto se debe a que, como se ha comentado antes, ambos reactivos fomentan las reacciones entre las cadenas de PLA, lo 
que ocasiona un aumento del peso molecular promedio del material y de su viscosidad intrínseca, que crece con el peso molecular y la ramificación.

En el caso del extensor de cadena, diversos trabajos, como los realizados por Tuna et al. [111] y Al-Itry et al. [3], señalan que los grupos epoxi presentes en los extensores de cadena pueden reaccionar, de distinta forma, con los grupos - $\mathrm{OH}$ y $-\mathrm{COOH}$ presentes en el PLA degradado. En el caso de los poliésteres, tal y como se muestra en la Fig. 53, los enlaces resultantes incluyen la apertura de los anillos epoxi del extensor, y la creación de enlaces covalentes con grupos hidroxilo y carboxilo. Los compuestos obtenidos pueden ser relativamente complejos, ya que son resultado de un balance entre la degradación, la extensión de cadena y la ramificación del PLA.

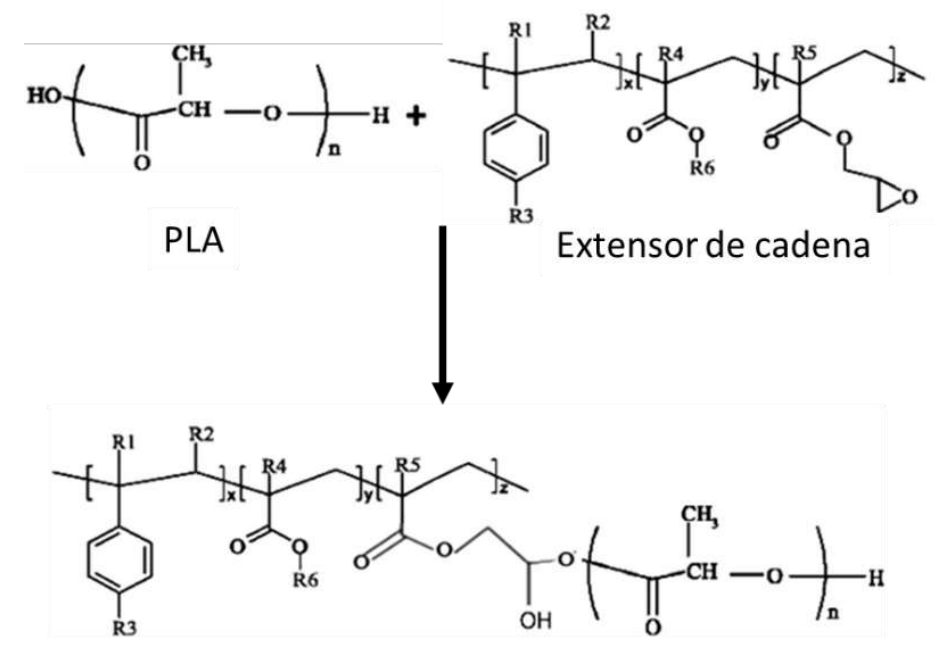

Fig. 53. Mecanismo de extensión de cadena del PLA con compuestos epoxi. Adaptado de [111].

En el caso del peróxido de dicumilo, el esquema de reacción se presenta en la Fig. 54. Como se puede observar, la reacción podría dividirse en tres etapas clave: en primer lugar, la generación de radicales primarios, consecuencia de la degradación térmica del peróxido; en segundo lugar, la abstracción de hidrógenos de las cadenas poliméricas por parte de los radicales primarios; y finalmente la reacción entre los dos radicales poliméricos para formar enlaces carbono - carbono [110]. 
Capítulo 6. Mejora de propiedades del PLA reciclado
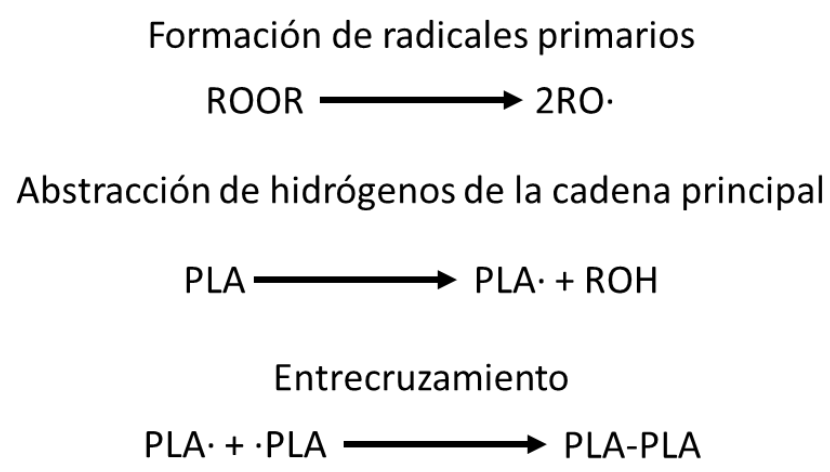

Fig. 54. Esquema de la reacción del PLA con peróxidos. Adaptado de [110].

Ambos mecanismos involucran la reacción de los grupos - $\mathrm{COOH}$ y/o $-\mathrm{OH}$ presentes en el PLA, que, como ya se ha visto mediante espectroscopía UV-Vis, se encuentran en mayor cantidad en los materiales reciclados. Este incremento en la cantidad de grupos reactivos es lo que convierte la adición de peróxidos y extensores de cadena en métodos efectivos para obtener un incremento de la viscosidad intrínseca del PLA reciclado mecánicamente.

\subsubsection{Efecto de la extrusión reactiva en la estructura del PLA reciclado}

Como se ha mencionado anteriormente, la estructura del PLA puede jugar un papel muy importante en las propiedades ópticas, mecánicas y de barrera del material reciclado, las cuales son cruciales en las aplicaciones de envasado. Con la finalidad de estudiar el efecto de los dos reactivos utilizados en la estructura del material reciclado se realizaron ensayos de espectroscopía FTIR y DSC de las diferentes muestras.

En la Fig. 55 se muestran los espectros FTIR del PLA extruido con diferentes aditivos. Como se puede observar, en líneas generales, los espectros de las diferentes muestras son muy parecidos, por lo que la extrusión reactiva no parece afectar de forma importante a la estructura química del PLA. 


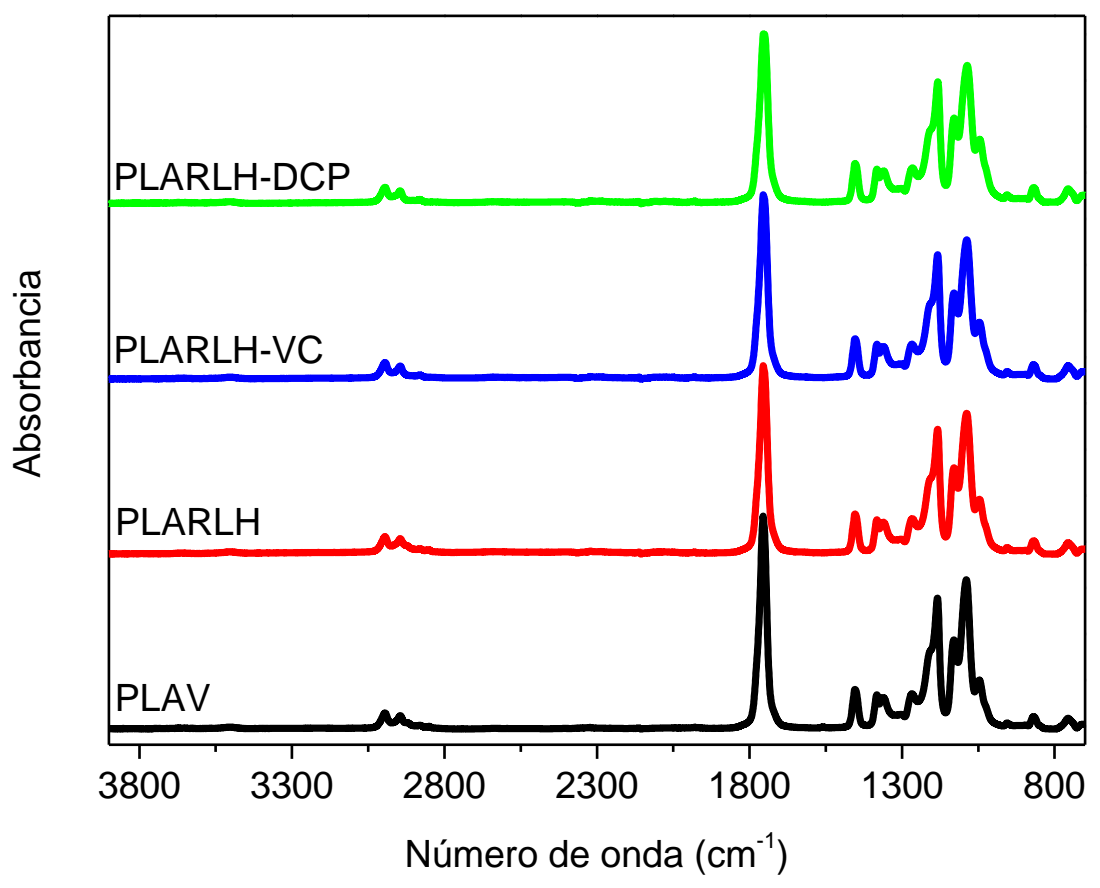

Fig. 55. Espectros FTIR del PLA reciclado mejorado mediante extrusión reactiva.

Sin embargo, si se observa detalladamente la banda centrada a $1755 \mathrm{~cm}^{-1}$, correspondiente a la tensión del grupo $\mathrm{C}=\mathrm{O}$, se pueden ver algunos cambios. La Fig. 56 muestra que tanto el PLARLH-VC (con extensor de cadena) como el PLARLH-DCP (con peróxido) muestran un comportamiento similar al del PLARLH, ya que se puede apreciar un ligero desplazamiento de la banda hacia menores números de onda, consecuencia de la presencia de nuevos grupos carbonílicos.

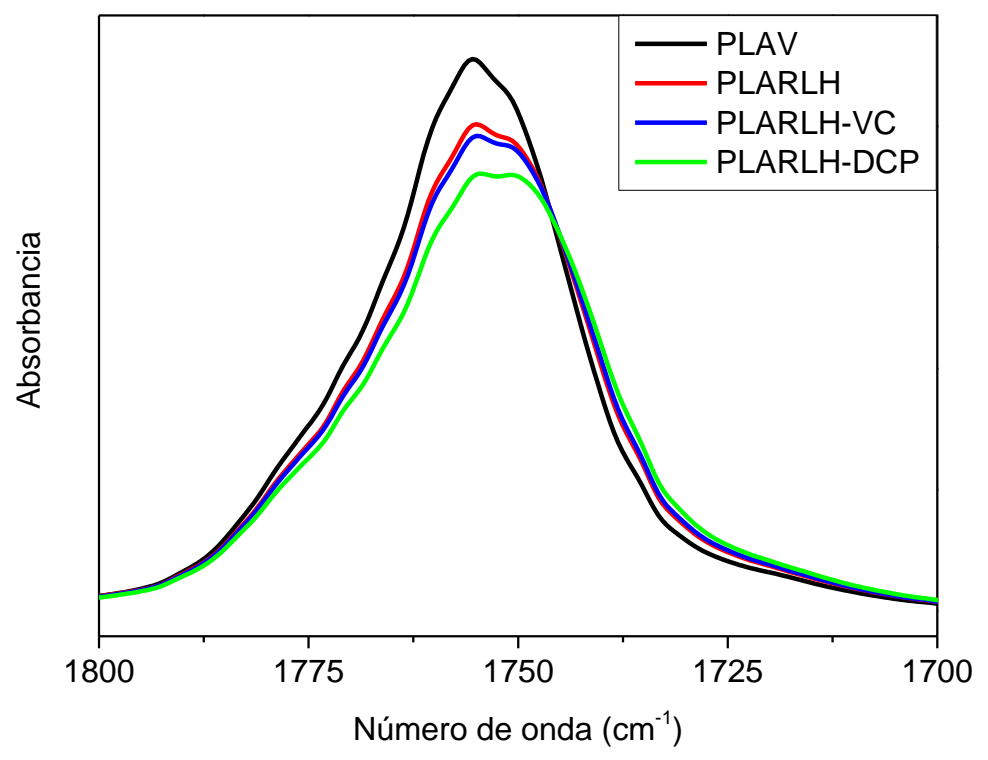

Fig. 56. Ampliación de la banda de $1750 \mathrm{~cm}^{-1}$ del PLA reciclado mejorado mediante extrusión reactiva. 
En cuanto a los ensayos de DSC, los resultados obtenidos se encuentran recogidos en la Fig. 57 y la Tabla 20. Se puede observar que, a simple vista, los barridos de calentamiento de los materiales mejorados mediante extrusión reactiva presentan diferencias con respecto al PLARLH. En primer lugar, se puede observar que la tanto el PLARLH-DCP como el PLARLH-VC presentan un valor de $T_{c c}$ menor que el PLARLH. Este resultado es bastante llamativo, ya que ambos materiales mejorados presentan una mayor viscosidad intrínseca, lo que indica que poseen cadenas más largas. Dichas cadenas deberían cristalizar con mayor dificultad, y por lo tanto aumentar la temperatura de cristalización en frío del PLA. Sin embargo, algunos autores, como Yang et al. [113] y Zhang et al. [120], señalan que la ligera ramificación de las cadenas de PLA promueve la formación de estructuras cristalinas en el PLA, ocasionando una disminución de la temperatura de cristalización enfrío.

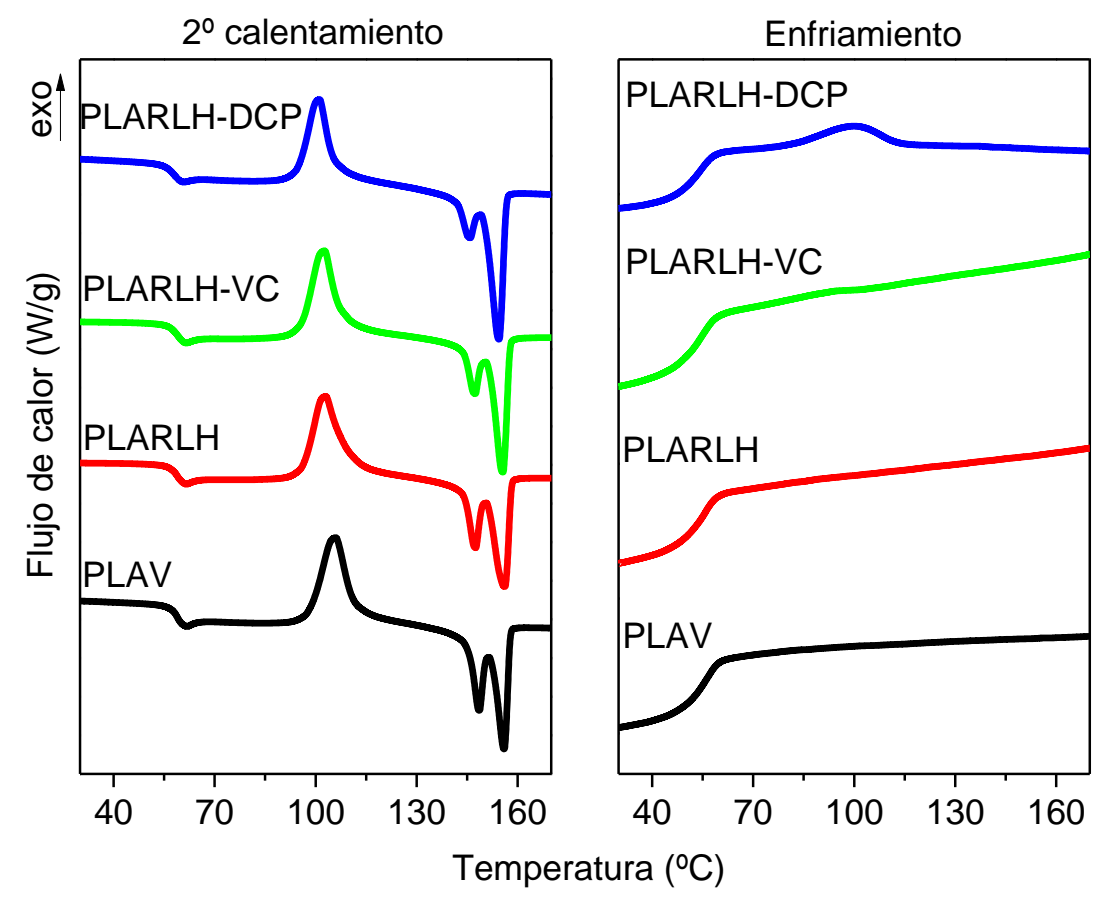

Fig. 57. Barridos de segundo calentamiento y de enfriamiento del PLA reciclado mejorado mediante extrusión reactiva.

En segundo lugar, se puede observar en la Tabla 20 que el PLARLH-DCP presenta una cristalinidad de $6 \%$, mientras que los otros materiales presentan valores muy cercanos a cero, lo que indica que el material con peróxido de dicumilo cristaliza durante el enfriamiento. Este resultado señala que la adición del peróxido de dicumilo aumenta la habilidad del PLA para formar estructuras cristalinas. Este comportamiento puede confirmarse al observar los barridos de enfriamiento de las diferentes muestras, mostrados en la Fig. 57, en los que se observa que el PLARLH-DCP es el único material que muestra un pico de cristalización.

En resumen, los resultados de FTIR y DSC nos indican que la adición de peróxido de dicumilo y de un extensor de cadena comercial no afectan en gran medida la estructura 
del PLA. Sin embargo, la ligera ramificación de las cadenas que producen esos reactivos facilita la cristalización del PLA, sobre todo en el caso del peróxido de dicumilo.

Tabla 20. Resultados de DSC (segundo barrido de calentamiento) del PLA reciclado mejorado mediante extrusión reactiva.

\begin{tabular}{cccccc}
\hline Material & $\begin{array}{c}\mathbf{T}_{\mathbf{c c}} \\
\left.\mathbf{(}^{\mathbf{o}} \mathbf{C}\right)\end{array}$ & $\begin{array}{c}\mathbf{T}_{\text {fus }} \\
\left({ }^{\mathbf{o}} \mathbf{C}\right)\end{array}$ & $\begin{array}{c}\Delta \mathbf{H}_{\text {crist }} \\
(\mathbf{J} / \mathbf{g})\end{array}$ & $\begin{array}{c}\Delta \mathbf{H}_{\text {fus }} \\
(\mathbf{J} / \mathbf{g})\end{array}$ & $\begin{array}{c}\mathbf{X}_{\mathbf{C}} \\
(\boldsymbol{\%})\end{array}$ \\
\hline PLAV & 106,0 & $148,6-156,0$ & 27,5 & 27,6 & 0 \\
PLARLH & 103,0 & $147,6-156,3$ & 27,4 & 28,3 & 1 \\
PLARLH-DCP & 101,2 & $146,0-154,5$ & 23,6 & 28,8 & 6 \\
PLARLH-VC & 102,6 & $147,5-155,6$ & 25,7 & 27,9 & 2 \\
\hline
\end{tabular}

\subsubsection{Efecto de la extrusión reactiva en la estabilidad térmica del PLA reciclado}

La utilización de diferentes aditivos podría ocasionar variaciones en las propiedades del PLA, entre ellas, la estabilidad térmica. En este trabajo se han realizado ensayos TGA de los diferentes materiales, y los resultados se encuentran resumidos en la Fig. 58 y la Tabla 21. Por una parte, se puede observar que la adición del PLA virgen y del extensor de cadena mejora de forma significativa la estabilidad térmica del PLA reciclado. De hecho, los valores de $T_{10}$ y $T_{\max }$ del PLARLH-VC, con extensor de cadena, se encuentran bastante próximos a los del PLAV. Esta mejora está relacionada con el incremento de la viscosidad intrínseca, ya que las cadenas más largas del mismo polímero se descomponen a una temperatura mayor.

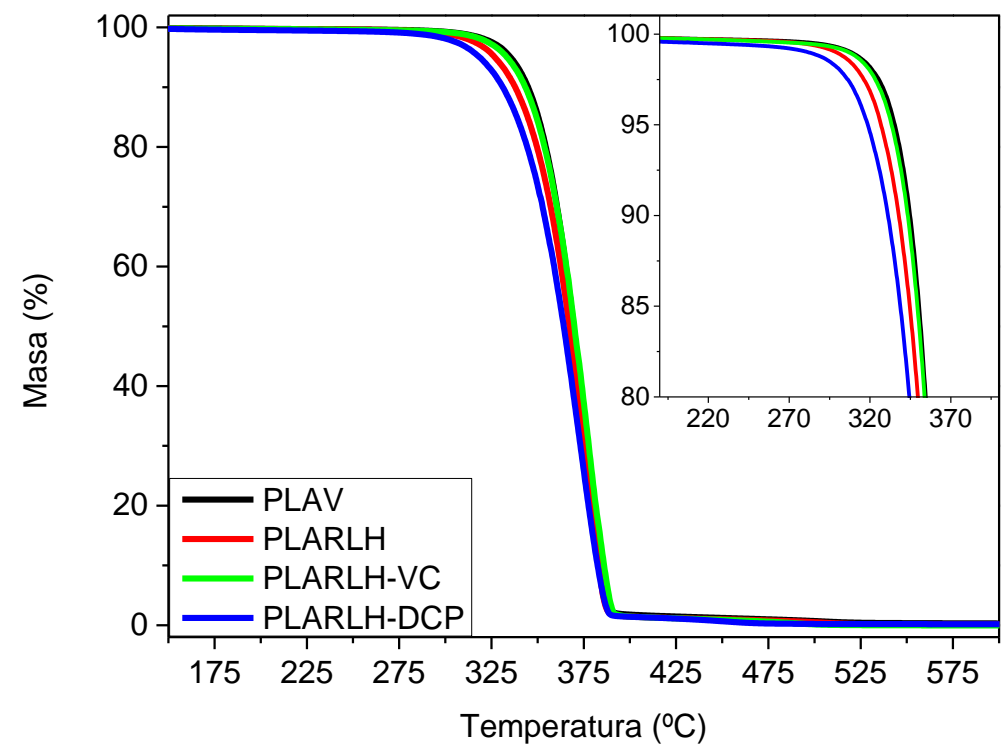

Fig. 58. Termogramas del PLA mejorado mediante extrusión reactiva. 
Por otra parte, se puede apreciar que la adición del peróxido de dicumilo afecta de forma negativa la estabilidad térmica del PLA reciclado, a pesar del alto valor de viscosidad intrínseca mostrado por este material. Este comportamiento podría ser debido a la presencia de cadenas poliméricas más cortas, consecuencia de la adición del peróxido, que no se hayan recombinado durante la extrusión reactiva y que se descompongan a una menor temperatura. La presencia de estas cadenas más cortas también podría catalizar la descomposición del PLA, ya que la mayor presencia de extremos de cadena promueve la depolimerización mediante transesterificación intramolecular durante el ensayo TGA, ya que este es el mecanismo predominante de descomposición entre 270 y $360^{\circ} \mathrm{C}$, tal y como han reportado Kopinke et al. al estudiar la descomposición térmica del PLA [60].

Estos resultados ponen de manifiesto la necesidad de estudiar cuidadosamente los aditivos empleados para la mejora de las prestaciones del PLA, ya que, a pesar de obtener incrementos en el peso molecular del polímero reciclado, otras propiedades podrían verse afectadas negativamente, disminuyendo el valor del material reciclado.

Tabla 21. Temperaturas características de TGA del PLA mejorado mediante extrusión reactiva.

\begin{tabular}{ccc}
\hline Muestra & $\mathbf{T}_{\mathbf{1 0}}\left({ }^{\mathbf{0}} \mathbf{C}\right)$ & $\mathbf{T}_{\mathbf{m a x}}\left({ }^{\mathbf{0}} \mathbf{C}\right)$ \\
\hline PLAV & 344,9 & 376,4 \\
PLARLH & 337,9 & 372,3 \\
PLARLH-VC & 343,2 & 377,4 \\
PLARLH-DCP & 331,2 & 372,8 \\
\hline
\end{tabular}

\subsubsection{Efecto de la extrusión reactiva en las propiedades ópticas del PLA reciclado}

Como se ha mencionado anteriormente, las propiedades ópticas juegan un papel crucial en las aplicaciones de envasado, las cuales representan el mercado más importante del PLA. Por ello, es importante determinar si la utilización de los diferentes aditivos para elevar el peso molecular afecta las propiedades ópticas del PLA. Con este objetivo se han realizado espectros UV-Vis de los diferentes materiales, cuyos resultados recogen la Fig. 59 y la Tabla 22. 


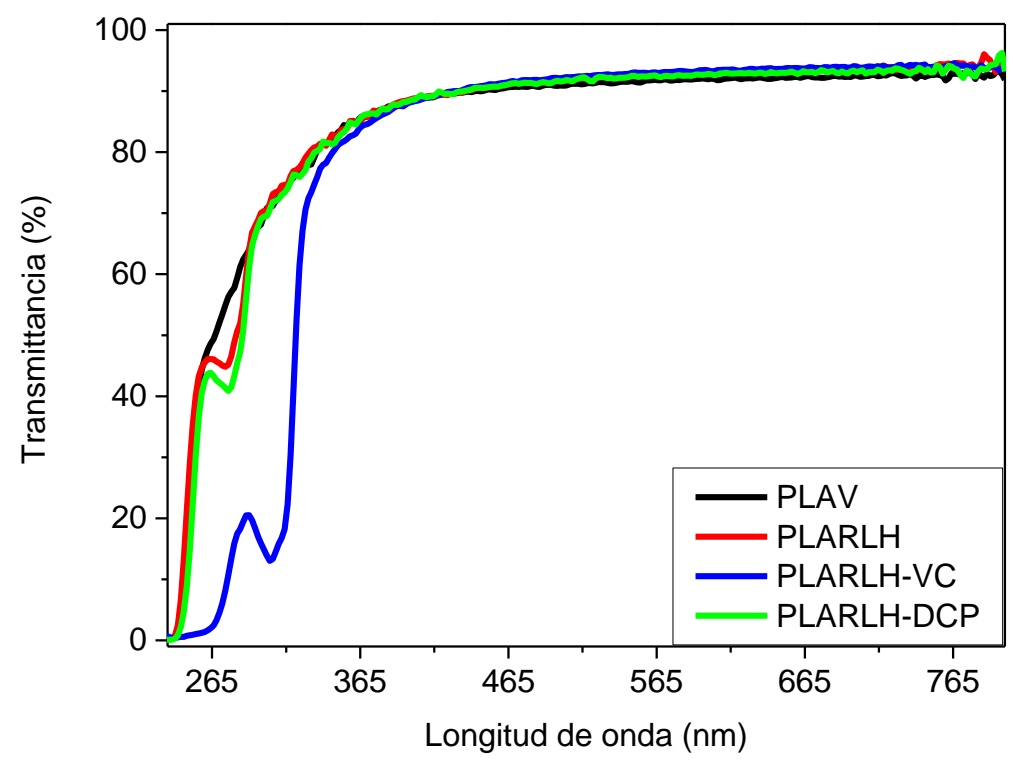

Fig. 59. Espectros UV-Vis del PLA mejorado mediante extrusión reactiva.

En la Fig. 59 se puede observar que el PLARLH-VC presenta un pico de absorción muy intenso, localizado alrededor de $305 \mathrm{~nm}$. Este pico se ha comprobado que corresponde al aditivo extensor, probablemente debido a la presencia de grupos aromáticos. Este resultado sugiere que la adición del extensor de cadena podría proporcionar cierto grado de protección frente a la radiación UV, tal y como sucede con la montmorillonita, lo cual es muy interesante para las aplicaciones en el envasado de alimentos. En cuanto a la muestra con el peróxido, se puede apreciar que esta presenta un pico de absorción alrededor de $275 \mathrm{~nm}$, al igual que el PLARLH. Este resultado indica que, a pesar del importante incremento de la viscosidad intrínseca, el PLARL-DCP cuenta con un importante número de grupos terminales carboxilo, tal y como sugieren los resultados del análisis termogravimétrico.

A pesar de las diferencias observadas en la región UV del espectro, la Tabla 22 muestra que todos los materiales presentan una buena transmisión de luz en la región visible del espectro. Este comportamiento se debe a la ausencia de cromóforos y a la muy escasa presencia de estructuras cristalinas, observada mediante DSC, que podrían actuar como centros de dispersión y reflexión de la luz. Estos resultados sugieren que, si bien los aditivos utilizados afectan el comportamiento del PLA en la región UV del espectro, los materiales mejorados presentan excelentes propiedades ópticas en la región visible, lo que es muy interesante desde el punto de vista de aplicaciones de envasado de alimentos.

Tabla 22. Transmisión de luz del PLA mejorado mediante extrusión reactiva.

\begin{tabular}{cc}
\hline Muestra & Transmisión de luz (\%) \\
\hline PLAV & 90,1 \\
PLARLH & 90,5 \\
\hline
\end{tabular}




\begin{tabular}{cc}
\hline PLARLH-VC & 91,4 \\
PLARLH-DCP & 90,1 \\
\hline
\end{tabular}

\subsubsection{Efecto de la extrusión reactiva en la microdureza del PLA reciclado}

Por último, se han realizado medidas de microdureza de las diferentes muestras de PLA mejorado mediante extrusión reactiva. En la Fig. 60 se puede observar que, aunque las diferencias son pequeñas y entran dentro de la incertidumbre de los resultados, la adición de peróxido y del extensor de cadena parecen ocasionar un incremento de la microdureza del PLA reciclado. En cualquier caso, se compensa el descenso que pudiera causar el reciclado mecánico. Este comportamiento está relacionado con el aumento del peso molecular observado en las medidas de viscosidad intrínseca. La recuperación de las propiedades mecánicas después del reciclado podría ser muy importante para la utilización de PLA reciclado en aplicaciones de envasado, lo que repercutiría positivamente en su reciclabilidad.

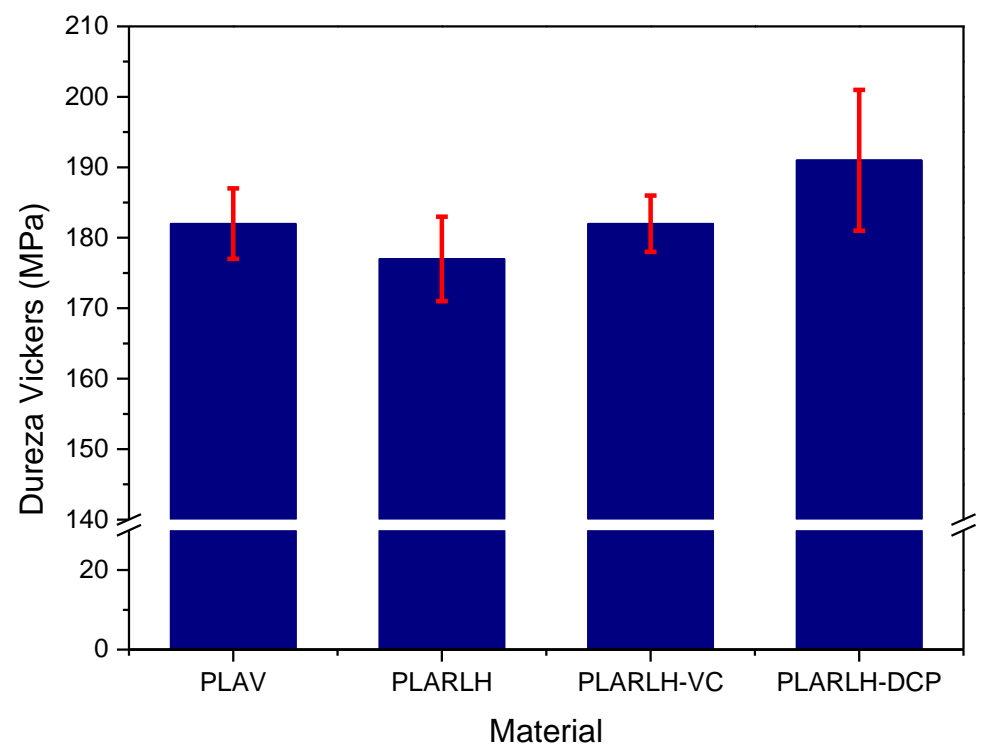

Fig. 60. Microdureza del PLA reciclado mejorado mediante extrusión reactiva

\subsection{Mejora de prestaciones de PLA reciclado mediante adición de arcillas}

La utilización de arcillas para mejorar las propiedades de diferentes matrices poliméricas ha sido ampliamente estudiada en la literatura. La presencia de estas partículas de escala nanométrica puede ocasionar una mejora en las propiedades mecánicas, térmicas y de barrera de los plásticos. Por ello, en este trabajo se emplea una montmorillonita orgánicamente modificada, una haloisita natural y una haloisita modificada como refuerzo del PLA reciclado. A continuación, se presentarán los resultados que permiten analizar el efecto de la adición de estas arcillas en las prestaciones del PLA reciclado. 


\subsubsection{Viscosidad intrínseca del PLA mejorado mediante adición de arcillas}

Como se ha mencionado anteriormente, la viscosidad intrínseca es un parámetro muy importante desde el punto de vista de procesabilidad del material reciclado, y podría desalentar el uso del PLA reciclado en algunas aplicaciones. La viscosidad intrínseca de las diferentes muestras de PLA reciclado mejorado con arcilla se muestra en la Fig. 61. Se puede observar que el PLARLH-C30, el PLARLH-Ha y especialmente el PLARLHHaM presentan una viscosidad superior a la del PLARLH, de manera que se recupera en buena parte la viscosidad perdida por la degradación en uso y el reprocesado. Este resultado es bastante llamativo, ya que, como se ha mencionado anteriormente, la presencia de arcillas cataliza la degradación del PLA durante el procesado. Sin embargo, algunos autores como Vassiliou et al. [112] señalan que los grupos -OH presentes en nanopartículas como la sílice o la montmorillonita pueden interactuar con los grupos -OH de los poliésteres, llevando a un incremento de la viscosidad intrínseca, y del peso molecular, del polímero. Un proceso similar puede haber sido sufrido por el PLARLH, ya que este material posee un mayor número de grupos - $\mathrm{OH}$ terminales, en comparación con el PLAV, que pueden interactuar con los grupos -OH de las arcillas.

Los resultados obtenidos sugieren que las arcillas podrían actuar como aditivos multifuncionales en el PLA reciclado, mejorando no sólo algunas propiedades del material, sino ayudando a elevar la viscosidad intrínseca del plástico.

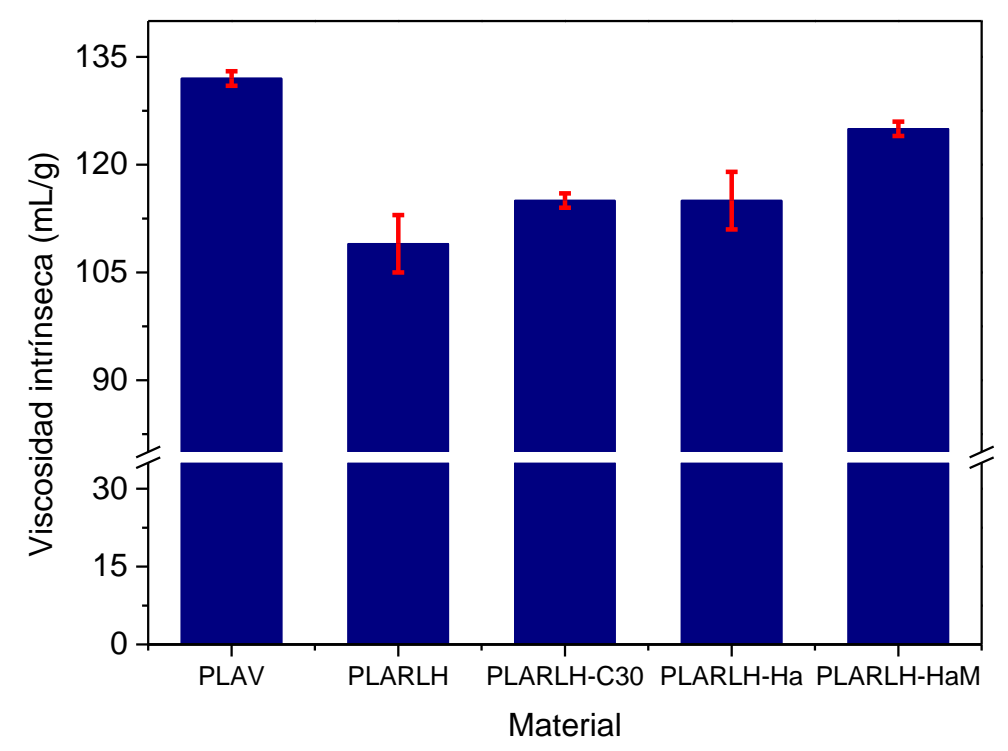

Fig. 61. Viscosidad intrínseca del PLA reciclado mejorado mediante adición de arcillas.

\subsubsection{Efecto de la adición de arcillas en la estructura del PLA reciclado}

Los cambios estructurales producidos en el PLA reciclado mejorado mediante la adición de arcillas han sido estudiados mediante espectroscopía FTIR y DSC. La Fig. 62 muestra los espectros FTIR del PLA reciclado mejorado con diferentes arcillas. Como se puede observar, no se aprecian diferencias importantes entre los materiales estudiados, 
indicando que la adición de las diferentes arcillas no modifica la estructura química del PLA. Sin embargo, otros autores, como Krishnaiah et al. [61] y Liu et al. [69], señalan que la adición de diferentes refuerzos, como la haloisita y la haloisita modificada con APS, ocasiona un incremento en las bandas de absorción ubicadas a 3504 y $3691 \mathrm{~cm}^{-1}$, como consecuencia de la interacción de los grupos $\mathrm{Si}-\mathrm{O}-\mathrm{Si}$ presentes en la arcilla con los grupos -OH del PLA. El hecho de que en nuestras muestras no se observe dicho comportamiento podría estar relacionado con la cantidad de haloisita utilizada, ya que en los estudios citados agregan entre 5 y $40 \%$ de arcilla, mientras que las muestras de este trabajo contienen sólo 2\%. Araujo et al. [5] reportó un resultado similar en nanocomposites de PLA con 3\% de $\mathrm{C} 30$, en los que no se aprecia ningún cambio estructural como consecuencia de la adición de la arcilla.

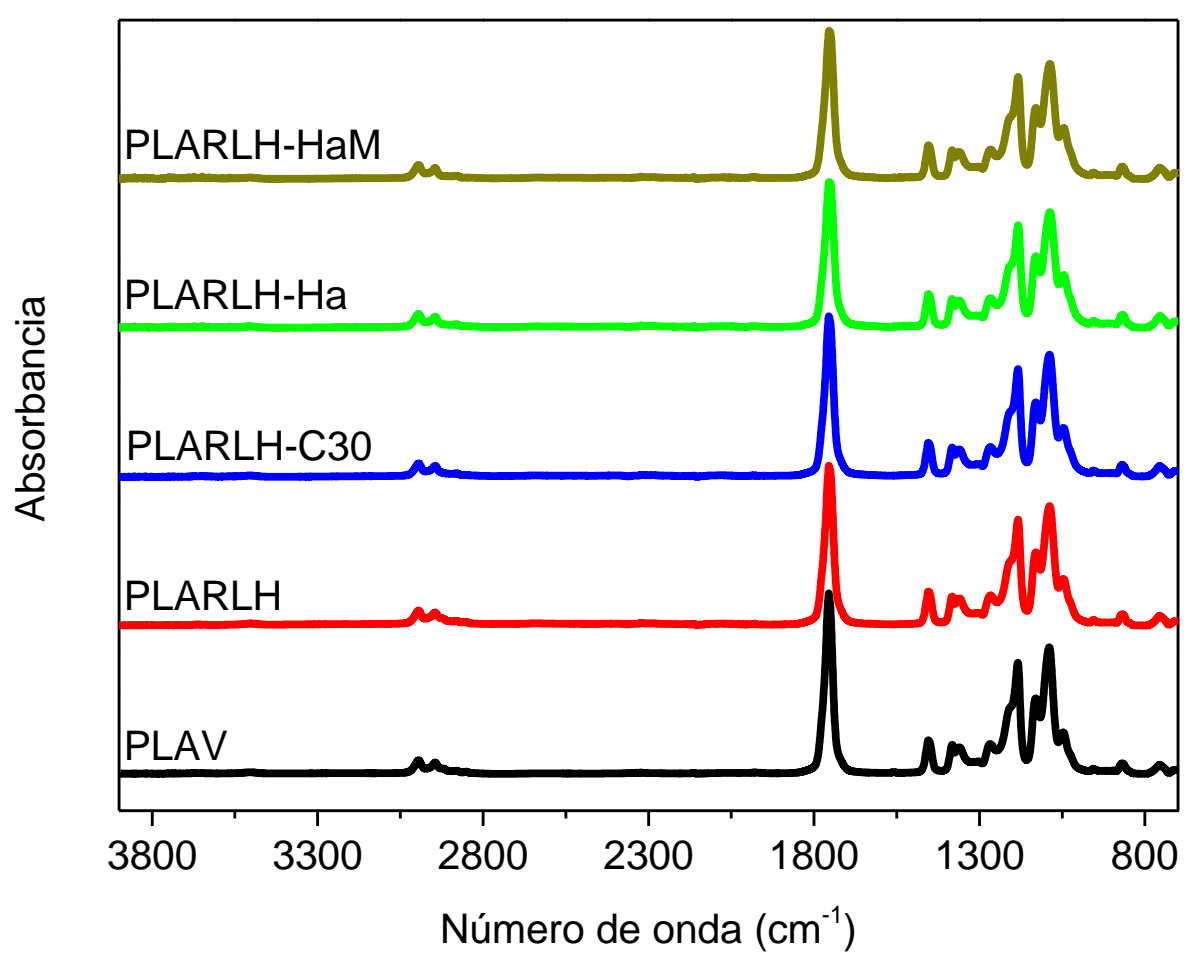

Fig. 62. Espectros FTIR del PLA reciclado mejorado mediante la adición de arcillas.

La Fig. 63 y la Tabla 23 recogen los resultados obtenidos a partir de los ensayos de DSC para las diferentes muestras de PLA reciclado mejorado con arcillas. El comportamiento de los diferentes materiales es muy similar entre sí, habiendo una pequeña diferencia sólo en los valores de $T_{c c}$. La adición de la montmorillonita ocasiona un leve descenso en la temperatura de cristalización, debido al efecto nucleante de esta arcilla en el PLA, que promueve la formación de estructuras cristalinas a una menor temperatura.

En cuanto al comportamiento de los materiales con haloisita, por una parte, podemos ver que la adición de la haloisita sin modificar produjo un leve descenso de la 
temperatura de cristalización, debido probablemente al efecto nucleante de las partículas de haloisita. Un resultado similar fue encontrado por Liu et al. [69] al estudiar el comportamiento térmico de nanocomposites de PLA con hasta $40 \%$ de haloisita. Por otra parte, la adición de la arcilla modificada con APS ocasiona un leve incremento de $T_{c c}$. Para explicar dicho aumento, hay que considerar que la modificación con APS disminuye el carácter hidrofílico de la haloisita, lo que daría lugar a fuertes interacciones con el PLA, tales como la formación de puentes de hidrógeno o incluso de enlaces covalentes entre el refuerzo y la matriz polimérica [25]. Estas interacciones entre el PLA y la haloisita modificada restringirían la movilidad de las cadenas poliméricas, dificultando la cristalización del PLA. Esta disminución de la movilidad de las cadenas poliméricas también fue observada por Krishnaiah et al. [61] en nanocomposites de PLA con haloisita modificada con APS.

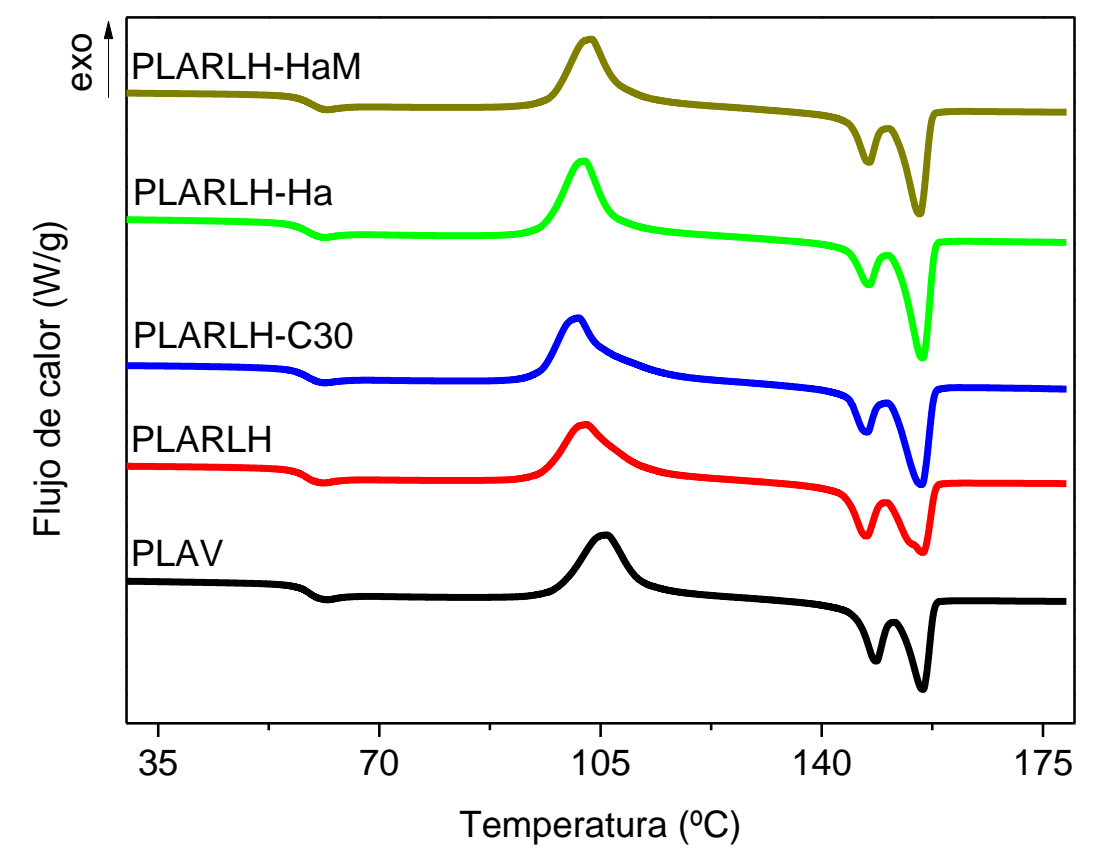

Fig. 63. Barridos de segundo calentamiento del PLA reciclado mejorado mediante adición de arcillas.

Resumiendo, los resultados obtenidos mediante FTIR y DSC sugieren que la adición de las arcillas no ocasiona cambios estructurales importantes en el PLA reciclado, lo que es muy importante ya que la estructura juega un papel primordial en algunas propiedades del PLA.

Tabla 23. Resultados de DSC (segundo barrido de calentamiento) del PLA reciclado mejorado mediante la adición de arcillas.

\begin{tabular}{ccccc}
\hline Material & $\mathbf{T}_{\text {cc }}$ & $\mathbf{T}_{\text {fus }}$ & $\Delta \mathbf{H}_{\text {crist }}$ & $\Delta \mathbf{H}_{\text {fus }}$ \\
& $\left({ }^{\circ} \mathbf{C}\right)$ & $\left({ }^{\circ} \mathbf{C}\right)$ & $(\mathbf{J} / \mathbf{g})$ & $(\mathbf{J} / \mathbf{g})$ \\
\hline
\end{tabular}




\begin{tabular}{ccccc}
\hline PLAV & 106,0 & $148,6-156,0$ & 27,5 & 27,6 \\
PLARLH & 103,0 & $147,6-156,3$ & 27,4 & 28,3 \\
PLARLH-C30 & 101,5 & $147,2-155,9$ & 28,6 & 29,2 \\
PLARLH-Ha & 102,5 & $147,6-156,0$ & 28,2 & 29,7 \\
PLARLH-HaM & 103,5 & $147,5-155,6$ & 27,5 & 27,6 \\
\hline
\end{tabular}

\subsubsection{Efecto de la adición de arcillas en la estabilidad térmica del PLA reciclado}

La estabilidad térmica del PLA reciclado mejorado mediante adición de arcillas fue estudiada mediante análisis termogravimétrico. Los resultados más importantes se encuentran recogidos en la Fig. 64 y la Tabla 24. Se puede observar que todas las muestras con arcillas presentan una mayor estabilidad térmica que el PLARLH, llegando a tener valores cercanos a los del PLAV. Este comportamiento se debe a que, como se ha mencionado en secciones previas, las partículas de arcilla actúan como una barrera que impide la liberación de los productos de descomposición del PLA, lo que aumenta la estabilidad térmica del material. Es importante destacar que las muestras con arcillas modificadas, C30 y HaM, presentan una mayor estabilidad que la muestra cuya arcilla se encuentra sin modificar. Esta diferencia podría ser debida a la mayor compatibilidad de las arcillas modificadas con el PLA, lo que ocasionaría una mejor dispersión de las arcillas $\mathrm{y}$, por lo tanto, una mayor estabilidad térmica.

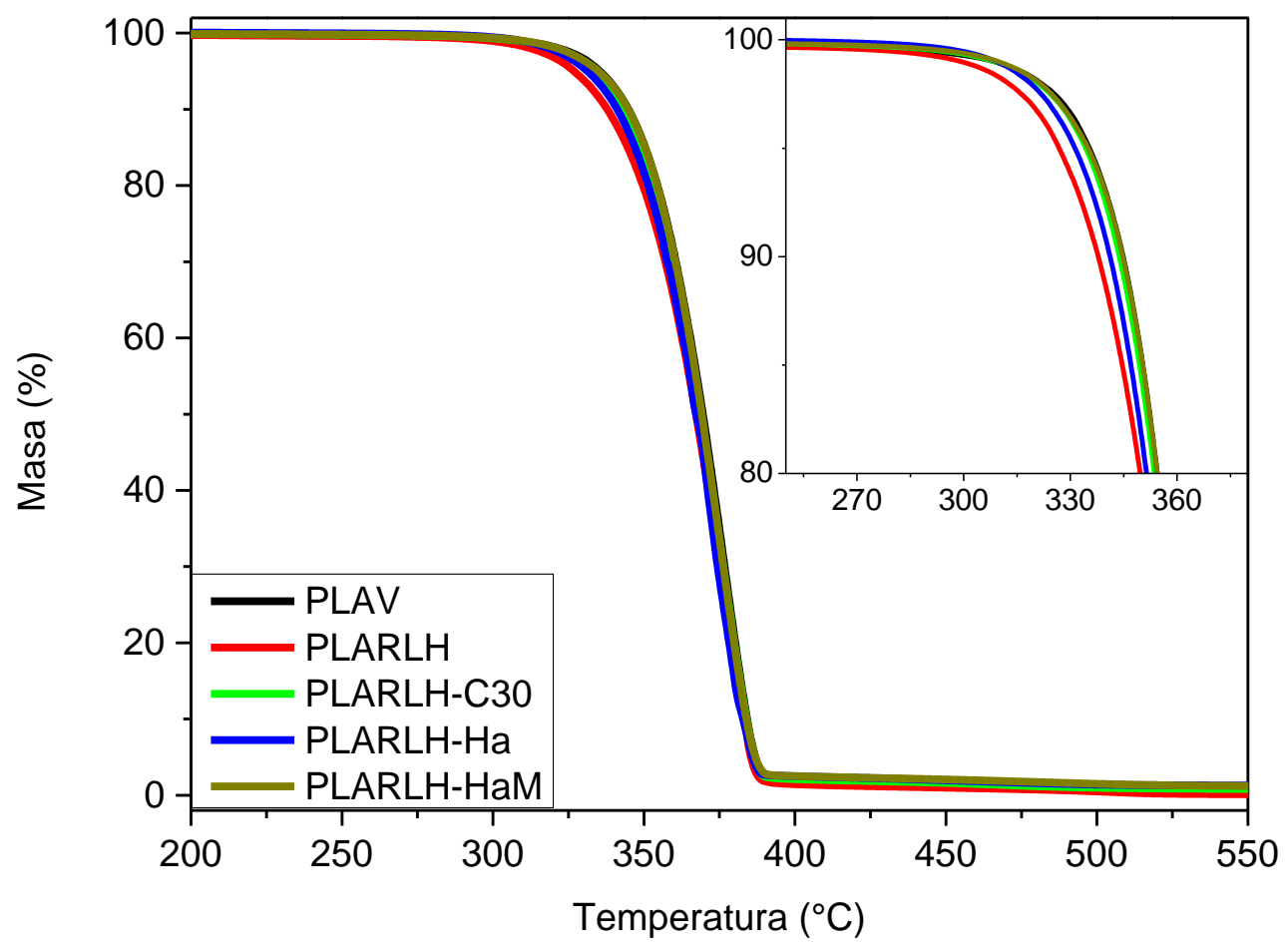

Fig. 64. Termogramas del PLA mejorado mediante adición de arcillas 
Sin embargo, en la sección 4.4 se ha observado que la adición de C30 y haloisita sin modificar tenía un efecto negativo sobre la estabilidad térmica del PLA virgen. Esta diferencia entre el efecto de las arcillas en el PLAV y el PLARLH puede ser debido a la mayor cantidad de grupos - $\mathrm{OH}$ presentes en el material reciclado. Estos grupos podrían ocasionar una mayor compatibilidad entre el polímero y las diferentes arcillas, lo que redunda en una mejor dispersión y mejores propiedades de los nanocomposites.

En cualquier caso, los resultados obtenidos indican que la adición de arcillas ayuda a mejorar la estabilidad térmica del PLA reciclado, obteniendo valores muy cercanos a los del polímero virgen al emplear arcillas modificadas orgánicamente.

Tabla 24. Temperaturas características de TGA del PLA mejorado mediante adición de arcillas.

\begin{tabular}{ccc}
\hline Muestra & $\mathbf{T}_{\mathbf{1 0}}\left({ }^{\mathbf{0}} \mathbf{C}\right)$ & $\mathbf{T}_{\mathbf{m a x}}\left({ }^{\mathbf{0}} \mathbf{C}\right)$ \\
\hline PLAV & 344,9 & 376,4 \\
PLARLH & 337,9 & 372,3 \\
PLARLH-C30 & 343,8 & 375,2 \\
PLARLH-Ha & 341,2 & 373,2 \\
PLARLH-HaM & 344,7 & 375,3 \\
\hline
\end{tabular}

\subsubsection{Efecto de la adición de arcillas en las propiedades ópticas del PLA reciclado}

Las propiedades ópticas de las muestras de PLA mejoradas mediante adición de arcillas fueron estudiadas mediante espectroscopía UV-Vis. En la Fig. 65 se puede observar que la adición de las arcillas modificadas afecta la absorción del PLA en la región UV, ya que el PLARLH-HaM y sobre todo el PLARLH-C30 presentan una mayor absorción en la región UV que el resto de los materiales. De hecho, en los materiales con arcilla modificada no es posible ver la banda de absorción ubicada alrededor de $275 \mathrm{~nm}$, característica del PLA reciclado, y que si se puede apreciar en el PLARLH-Ha. Este resultado podría ser interesante desde el punto de vista de aplicaciones de envasado de alimentos, ya que sugiere que la adición de las arcillas modificadas provee protección frente a la radiación UV. 


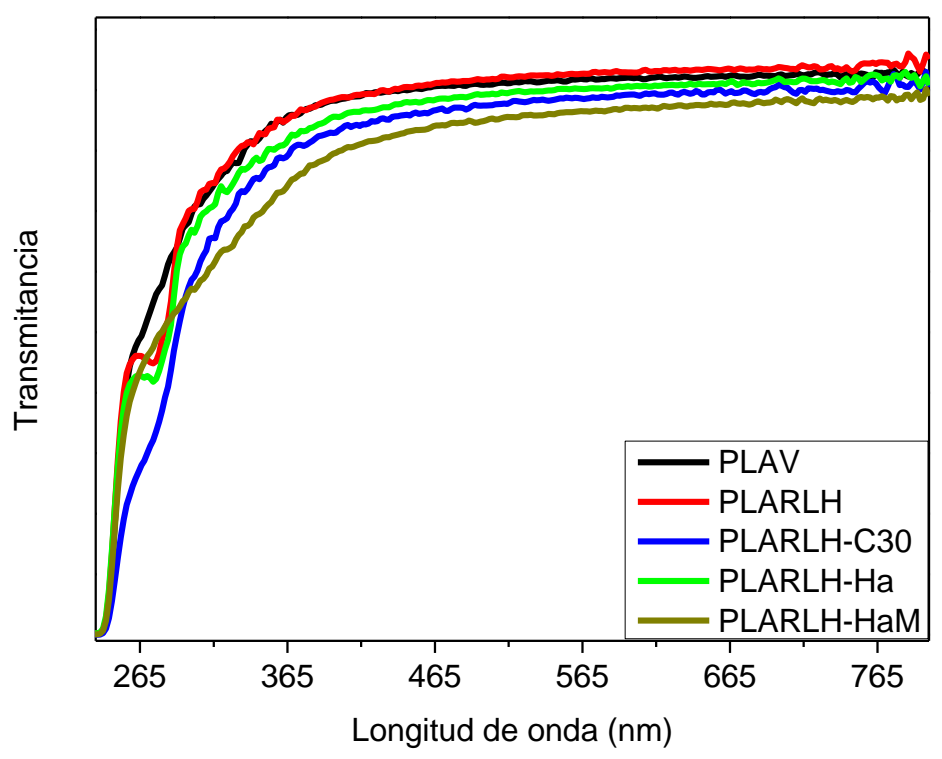

Fig. 65. Espectros UV-Vis del PLA reciclado mejorado mediante adición de arcillas.

En cuanto a la transmisión de luz, que está relacionada con la claridad óptica, la Tabla 25 muestra que la adición de los refuerzos ocasiona una ligera disminución en la transmisión de luz del PLA reciclado, debido a que las partículas de arcilla dispersas en la matriz polimérica actúan como centros de dispersión y reflexión de la luz, disminuyendo la transmisión de luz de los nanocomposites. Sin embargo, los cambios son bastante pequeños, obteniendo una transmisión de luz que supera el $85 \%$ en todos los casos. Por lo tanto, se puede concluir que la adición de las diferentes arcillas no solo no afecta de forma importante la claridad óptica del PLA reciclado, sino que además puede proveer otras funcionalidades, como la protección frente a la radiación UV.

Tabla 25. Transmisión de luz del PLA reciclado mejorado mediante adición de arcillas.

\begin{tabular}{cc}
\hline Muestra & Transmisión de luz (\%) \\
\hline PLAV & 90,1 \\
PLARLH & 90,5 \\
PLARLH-C30 & 86,3 \\
PLARLH-Ha & 88,2 \\
PLARLH-HaM & 85,8 \\
\hline
\end{tabular}

\subsubsection{Efecto de la adición de arcillas en la microdureza del PLA reciclado}

Finalmente, también se realizaron medidas de microdureza de las muestras de PLA reciclado mejorado mediante la adición de arcillas, cuyos resultados se pueden observar en la Fig. 66. Las diferentes arcillas ocasionan un aumento de la dureza del PLA 
reciclado, llegándose a obtener valores ligeramente superiores a los del PLAV, lo que pone de manifiesto el efecto de refuerzo que ejercen las partículas de arcilla.

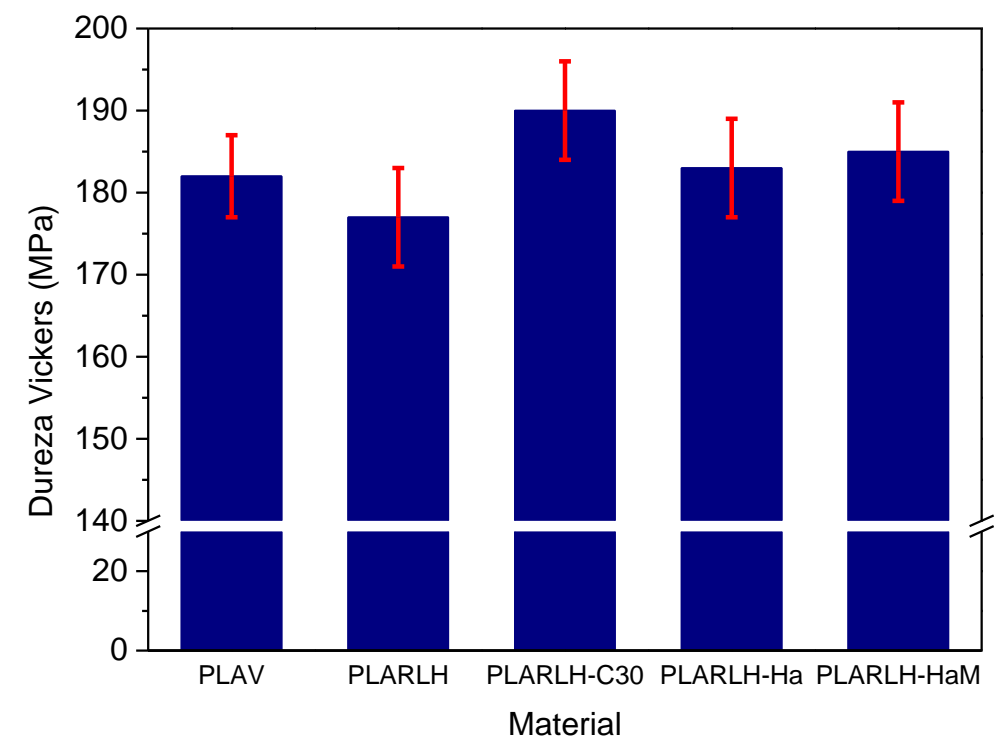

Fig. 66. Microdureza del PLA reciclado mejorado mediante adición de arcillas.

Los resultados muestran que PLARLH-HaM y, sobre todo, el PLARLH-C30 presentan los valores de dureza más altos. Otros autores, como Krishnaiah et al. [61], han encontrado resultados similares al estudiar las propiedades mecánicas de nanocomposites de PLA con haloisita modificada con APS. Este comportamiento se debe a la modificación orgánica presente en ambas arcillas, y que ocasiona un incremento de las interacciones entre el polímero y las partículas, lo que conduce a materiales con mejores propiedades mecánicas. 


\section{CONCLUSIONES Y TRABAJOS FUTUROS}

\subsection{Conclusiones}

El mercado de los bioplásticos ha aumentado de forma importante durante los últimos años y todas las previsiones indican que seguirá creciendo mucho en el futuro. Uno de los bioplásticos más importantes es el poli(ácido láctico), polilactida o PLA, debido a que es una de las alternativas más interesantes para sustituir a los plásticos basados en combustibles fósiles en algunas aplicaciones, como el envasado de alimentos frescos. Sin embargo, el consumo masivo del PLA puede traer consigo varios problemas de índole social y medioambiental: en primer lugar, el abandono de la producción de alimentos en favor de los cultivos utilizados como materia prima en la producción del PLA puede amenazar la sostenibilidad de los países en vías de desarrollo; en segundo lugar, el uso masivo y la lenta velocidad de degradación de algunos grados de PLA podría ocasionar una importante acumulación de residuos en los próximos años. Estos problemas han despertado el interés, a nivel científico e industrial, de estudiar alternativas para la valorización de los residuos de PLA. Entre estas alternativas destaca, desde un punto de vista medioambiental, el reciclado mecánico.

Este trabajo ha tenido dos objetivos principales. El primero ha sido estudiar los efectos de la degradación en uso y de los procesos de reciclado mecánico sobre la estructura y propiedades del plástico de postconsumo reciclado mecánicamente. Para ello se han sometido muestras de PLA a diferentes procesos de envejecimiento acelerado y de reciclado mecánico, y se han estudiado los efectos en el peso molecular y en algunas propiedades interesantes desde el punto de vista de aplicaciones de envasado. El estudio se ha realizado también con nanocomposites con montmorillonita y haloisita. Los materiales vírgenes y reciclados fueron caracterizados empleando diferentes técnicas experimentales, que incluyen: viscosimetría, XRD, TEM, SEM, espectroscopía FTIR, DSC, TGA, espectroscopía UV-Vis, medidas de microdureza, permeabilidad frente a diferentes gases y ensayos de degradación hidrolítica. Las principales conclusiones obtenidas en este bloque se enumeran a continuación: 
- Los diferentes procesos de reciclado causan la degradación del PLA. La reducción del peso molecular depende del proceso de reciclado utilizado, ya que las muestras sometidas a la etapa de lavado presentan una menor viscosidad intrínseca.

- Además de la degradación del PLA, el reciclado afecta la dispersión de las nanopartículas de arcilla en los nanocomposites. Los ensayos de XRD y TEM muestran que tanto la C30 como la haloisita se encuentran mejor dispersas en la matriz de PLA en los materiales reciclados.

- Los espectros FTIR del PLA virgen y de los diferentes reciclados son muy similares, aunque existe una pequeña diferencia en la banda correspondiente a la tensión de los grupos $\mathrm{C}=\mathrm{O}\left(1755 \mathrm{~cm}^{-1}\right)$. Esta diferencia se debe a la formación de compuestos carbonílicos como consecuencia de la degradación del PLA durante el reciclado.

- Los ensayos de DSC y XRD muestran que el reciclado no modifica el carácter amorfo del PLA. Sin embargo, la degradación del PLA aumenta la movilidad de las cadenas poliméricas, lo que se traduce en una disminución de la temperatura de cristalización en frío y en un incremento del pico de fusión de alta temperatura.

- Los ensayos de TGA muestran que el reciclado mecánico ocasiona una ligera disminución de la estabilidad térmica. Esta disminución es menor en los nanocomposites, debido a que la mejor dispersión de la arcilla contribuye a contrarrestar el efecto negativo de la degradación del PLA.

- Los espectros UV-Vis muestran la aparición de un pico de absorción en los materiales reciclados, ubicado alrededor de $275 \mathrm{~nm}$. Este pico puede ser debido a la presencia de grupos - $\mathrm{COOH}$ como consecuencia de la degradación del PLA durante el reciclado. Además, los diferentes procesos de reciclado no afectan la transmisión de luz en la región visible del espectro de los diferentes materiales.

- Los diferentes procesos de reciclado causaron una ligera disminución en la dureza del PLA, como consecuencia de la degradación del polímero. Este descenso fue más pequeño en los nanocomposites, ya que la mejor dispersión de la arcilla contrarresta, en parte, el efecto negativo del descenso del peso molecular del PLA.

- La permeabilidad del PLA frente al $\mathrm{O}_{2}, \mathrm{~N}_{2}$ y $\mathrm{CO}_{2}$ se ve afectada negativamente por el reciclado mecánico. Esto se debe a la degradación del PLA, ya que las cadenas más cortas ocasionan un mayor volumen libre dentro del material, lo que facilita la difusión de las moléculas de gas a través del polímero. En los nanocomposites el comportamiento es diferente, ya que los materiales reciclados presentan una menor permeabilidad frente a los gases que los materiales vírgenes. Esto se debe a la mejora de la dispersión de la arcilla, que actúa como una barrera que dificulta la difusión de las moléculas de gas a través del polímero.

- En cuanto al efecto del reciclado en la cinética de absorción de agua, los resultados señalan que, a $37{ }^{\circ} \mathrm{C}$, el reciclado mecánico causa un ligero aumento del coeficiente de difusión. Esto se debe a que hay un mayor volumen libre dentro del material como consecuencia de la degradación durante el reciclado.

- La absorción de agua en las primeras etapas puede describirse satisfactoriamente mediante un modelo Fickiano. No sucede lo mismo a tiempos elevados debido a los 
procesos de relajación, hinchamiento y degradación hidrolítica que favorecen la absorción de agua.

- A $58{ }^{\circ} \mathrm{C}$ se observa un incremento significativo de la velocidad en el proceso de absorción, debida a la mayor movilidad de las cadenas poliméricas. Los resultados parecen indicar que a $58^{\circ} \mathrm{C}$ el efecto del reciclado es menos importante que el efecto de la temperatura en la absorción de agua.

- En cuanto a la resistencia frente a la degradación hidrolítica, los resultados de viscosidad intrínseca muestran que, tanto a 37 como a $58^{\circ} \mathrm{C}$, el reciclado no parece afectar de manera significativa la velocidad de degradación del PLA o sus nanocomposites. Un comportamiento similar puede ser observado en los resultados de TGA, en los que la disminución de la estabilidad térmica durante la inmersión es similar en todos los materiales.

- La liberación de productos de la degradación hidrolítica del PLA al medio de inmersión puede ser seguida, al menos de forma cualitativa, empleando espectroscopía UV-Vis. Se pudo observar la aparición de diferentes bandas de absorción atribuidas a la presencia de diferentes productos de degradación en el medio.

- La inmersión a $37^{\circ} \mathrm{C}$ ocasionó pequeños cambios estructurales en el PLA y los nanocomposites con C30 y haloisita. Después de 84 días de inmersión se observó una cristalización naciente en todas las muestras. Estos cambios son ligeramente más grandes en los materiales reciclados.

- La aceleración del proceso de degradación a $58^{\circ} \mathrm{C}$ también se vio reflejada en la estructura de los materiales sumergidos. Después de sólo 13 días de inmersión se observaron grados de cristalinidad superiores al $30 \%$ en todos los casos.

- Mediante FTIR, XRD y DSC se determinó que a $58^{\circ} \mathrm{C}$ se forma una mezcla de dos fases cristalinas, $\alpha$ y $\alpha^{\prime}$, en el PLA. Además, tanto en el PLA puro como en el nanocomposite con haloisita el contenido de la fase $\alpha$ aumenta durante la inmersión, mientras que la presencia de la C30 parece inhibir la formación de cristales $\alpha$.

- La formación de estructuras cristalinas y el envejecimiento físico sufrido por las muestras sumergidas 37 y $58{ }^{\circ} \mathrm{C}$ ocasionaron un incremento en los valores de dureza de los diferentes materiales, a pesar de la degradación hidrolítica del PLA.

- Globalmente se puede decir que cabe esperar que un PLA de postconsumo sometido a reciclado mecánico muestre una ligera degradación. El descenso en las propiedades clave de cara al envasado es pequeño y el material reciclado podría tener prestaciones suficientes para ser utilizado nuevamente, incluso en aplicaciones de envasado.

En cualquier caso, los diferentes procesos de reciclado ocasionan una cierta degradación y pérdida de propiedades del PLA. La regradación económica el material reciclado, recuperando las propiedades perdidas, supondría un mayor valor del material reciclado y aumentaría la reciclabilidad del PLA. Por ello, el segundo objetivo principal de este trabajo ha sido la evaluación de diferentes métodos económicos para la obtención de mejores propiedades en el PLA reciclado mecánicamente. Se tomó como material reciclado de referencia un PLA sometido a un primer procesado, un envejecimiento acelerado con etapas de degradación fotoquímica, térmica e hidrotérmica, una etapa de lavado y un segundo reprocesado, en el cual fueron probadas las diferentes alternativas 
para la mejora de las propiedades del PLA. Los métodos empleados pueden dividirse en dos categorías: la extrusión reactiva, dentro de la que se incluye la adición de peróxido de dicumilo y la mezcla con PLA virgen y un extensor de cadena comercial; y la adición de arcillas, concretamente Cloisite $\mathrm{C} 30 \mathrm{~B}$, haloisita natural y haloisita orgánicamente modificada. Los materiales obtenidos fueron estudiados utilizando viscosimetría, espectroscopía FTIR, DSC, TGA, espectroscopía UV-Vis y medidas de microdureza. A partir de los resultados obtenidos se pueden extraer las siguientes conclusiones:

- La adición del peróxido de dicumilo y del extensor de cadena comercial produce un incremento en la viscosidad intrínseca del PLA reciclado, debido a las reacciones de entrecruzamiento y de extensión de cadena que tienen lugar durante la extrusión. La adición de arcillas aumenta ligeramente la viscosidad del PLA reciclado, lo cual puede ser debido a la interacción entre los grupos -OH de las arcillas y del PLA.

- La adición del extensor de cadena y, sobre todo, del peróxido de dicumilo ocasiona la formación de pequeñas ramificaciones, que favorecen la formación de estructuras cristalinas en el PLA. De hecho, el material con peróxido de dicumilo es el único de los estudiados que presenta cristalización durante el enfriamiento.

- Los resultados de DSC indican que la adición de la Cloisite C30B y la haloisita natural favorece ligeramente la cristalización en frío del PLA, debido al carácter nucleante de estas arcillas. Sin embargo, la adición de la haloisita modificada no afecta de forma importante la capacidad para cristalizar del PLA, debido a las fuertes interacciones entre esta arcilla y el polímero.

- Por una parte, la adición del extensor de cadena y de las diferentes arcillas conduce a una mejora de la estabilidad térmica del PLA reciclado, llegando a alcanzar valores de $T_{10}$ similares a los del PLA virgen. Por otra parte, la adición del peróxido de dicumilo afecta negativamente la estabilidad térmica del PLA reciclado, debido a la presencia de algunas cadenas poliméricas más cortas que pueden catalizar la descomposición térmica del PLA.

- Los espectros UV-Vis muestran que la adición del peróxido de dicumilo y del extensor de cadena comercial no afecta de manera importante la transmisión de luz del PLA reciclado en la región visible del espectro. Además, la presencia del extensor de cadena podría proveer cierto grado de protección frente a la radiación UV.

- La adición de las diferentes arcillas disminuye ligeramente la transmisión de luz del PLA reciclado en la región visible del espectro, debido a que las partículas de arcilla actúan como centros reflectores y difusores de la luz.

- Los valores de microdureza de las muestras con peróxido de dicumilo y el extensor de cadena son ligeramente superiores a los del PLA reciclado, indicando que la adición de estos compuestos permite recuperar parcialmente las propiedades mecánicas del PLA reciclado. En cuanto al efecto de las arcillas en la microdureza, los resultados indican que la modificación de la arcilla produce un material con mejores propiedades mecánicas, aunque en todos los casos se observó un incremento de la dureza en comparación con el PLA reciclado. 
En resumen, los resultados de este estudio indican que es posible obtener PLA reciclado con mejores propiedades añadiendo aditivos o refuerzos durante el reprocesado. Esta mejora de las prestaciones del PLA reciclado contribuye a mejorar la reciclabilidad de este bioplástico, disminuyendo el consumo de recursos y energía, y ayudando a mantener el bajo impacto medioambiental del PLA.

\subsection{Trabajos futuros y recomendaciones}

Como se ha mencionado anteriormente, es posible mejorar las propiedades del PLA reciclado mediante diferentes métodos. Las alternativas estudiadas en este trabajo son sólo algunas de las que se encuentran disponibles para mejorar las prestaciones del material reciclado, por lo que se recomienda un estudio más profundo de la mejora de las propiedades del PLA reciclado, que podría incluir:

- Añadir diferentes porcentajes de extensores de cadena y agentes de entrecruzamiento, como el trifenilfosfito, en las propiedades del PLA reciclado.

- Utilizar otros refuerzos derivados de fuentes renovables, como la celulosa nanocristalina y la queratina, para mejorar las prestaciones del PLA reciclado.

- Utilizar refuerzos derivados del carbono, como el grafeno o nanotubos de carbono, para obtener un PLA reciclado con mejores propiedades.

- Estudiar la polimerización en estado sólido (SSP) del PLA reciclado, con la finalidad de aumentar su peso molecular.

Los estudios sugeridos implican determinar el peso molecular, la estructura, las propiedades térmicas, ópticas, mecánicas y de barrera de los materiales obtenidos para evaluar así su reciclabilidad. 


\section{REFERENCIAS}

1. Commission Regulation (EU) No 10/2011 of 14 January 2011 on plastic materials and articles intended to come into contact with food. OJ L 12.

2. Aeschelmann F, Carus M: Biobased Building Blocks and Polymers: Global Capacities and Trends 2016-2021. Hürth, Germany, nova-Institüt, 2017

3. Al-Itry R, Lamnawar K, Maazouz A. Improvement of thermal stability, rheological and mechanical properties of PLA, PBAT and their blends by reactive extrusion with functionalized epoxy. Polymer Degradation and Stability.

DOI:https://doi.org/10.1016/j.polymdegradstab.2012.06.028

4. Al-Salem SM, Lettieri P, Baeyens J. Recycling and recovery routes of plastic solid waste (PSW): A review. Waste Management.

DOI:https://doi.org/10.1016/j.wasman.2009.06.004

5. Araujo A, Botelho Gy, Oliveira M, Machado AV. Influence of clay organic modifier on the thermal-stability of PLA based nanocomposites. Appl Clay Sci. 2014;88-89:144150

6. Arranz-Andrés J, Lorenzo V, de la Orden MU, Pérez E, Cerrada ML. Tailoring transport properties in blends based on olephinic and liquid crystalline polymers. J Membr Sci. 2011;377:141-150 
7. Arrieta PM, Samper DM, Aldas M, LÃpez J. On the Use of PLA-PHB Blends for Sustainable Food Packaging Applications. Materials. 2017;10

8. Auras R, Lim L, Selke SEM, Tsuji H: Poly(Lactic Acid): Synthesis, Structures, Properties, Processing, and Applications. New Jersey, John Wiley \& Sons, 2010

9. Auras RA, Singh SP, Singh JJ. Evaluation of oriented poly(lactide) polymers vs. existing PET and oriented PS for fresh food service containers. Packaging Technology and Science. 2005;18:207-216

10. Auras R, Harte B, Selke S. An Overview of Polylactides as Packaging Materials. Macromolecular Bioscience. 2004;4:835-864

11. Badia JD, Gil-Castell O, Ribes-Greus A. Long-term properties and end-of-life of polymers from renewable resources. Polym Degrad Stab. 2017;137:35-57

12. Badia JD, Ribes-Greus A. Mechanical recycling of polylactide, upgrading trends and combination of valorization techniques. European Polymer Journal. 2016;84:22-39

13. Badia JD, Santonja-Blasco L, Martínez-Felipe A, Ribes-Greus A. Hygrothermal ageing of reprocessed polylactide. Polym Degrad Stab. 2012;97:1881-1890

14. Badia JD, Strömberg E, Karlsson S, Ribes-Greus A. Material valorisation of amorphous polylactide. Influence of thermo-mechanical degradation on the morphology, segmental dynamics, thermal and mechanical performance. Polym Degrad Stab. 2012;97:670-678 
15. Badía JD, Strömberg E, Ribes-Greus A, Karlsson S. Assessing the MALDI-TOF MS sample preparation procedure to analyze the influence of thermo-oxidative ageing and thermo-mechanical degradation on poly (Lactide). Eur Polym J. 2011;47:1416-1428

16. Beltrán FR, Ortega E, Solvoll AM, Lorenzo V, de 1O, Martínez Urreaga J. Effects of Aging and Different Mechanical Recycling Processes on the Structure and Properties of Poly(lactic acid)-clay Nanocomposites. Journal of Polymers and the Environment. 2017

17. Beltrán FR, de la Orden MU, Lorenzo V, Pérez E, Cerrada ML, Martínez Urreaga J. Water-induced structural changes in poly(lactic acid) and PLLA-clay nanocomposites. Polymer. 2016;107:211-222

18. Beltrán FR, Lorenzo V, Acosta J, de la Orden MU, Martínez Urreaga J. Effect of simulated mechanical recycling processes on the properties of poly(lactic acid). $\mathbf{J}$ Environ Manag. In press

19. Beltrán FR, Lorenzo V, de la Orden MU, Martínez-Urreaga J. Effect of different mechanical recycling processes on the hydrolytic degradation of poly(l-lactic acid). Polym Degrad Stab. 2016;133:339-348

20. Bocchini S, Frache A. Comparative study of filler influence on polylactide photooxidation. Express Polym Lett. 2013;7:431-442

21. Bouzouita A, Notta-Cuvier D, Raquez J, Lauro F, Dubois P. Poly(lactic acid)-Based Materials for Automotive Applications, in Abe A, Albertsson A-, Coates G, Genzer J, Kobayashi S, Lee K-, Leibler L, Long TE, Möller M, Okay O, Percec V, Tang BZ, Terentjev EM, Theato P, Vincent MJ, Voit B, Wiesner U, Zhang X, editors. Advances in Polymer Science. Berlin, Heidelberg, Springer Berlin Heidelberg, 2017, pp 1-43 
22. Bragg WH, Bragg WL. The reflection of X-rays by crystals. Proc R Soc Lond A Math Phys Sci. 1913;88:428

23. Brüster B, Addiego F, Hassouna F, Ruch D, Raquez J-, Dubois P. Thermomechanical degradation of plasticized poly(lactide) after multiple reprocessing to simulate recycling: Multi-scale analysis and underlying mechanisms. Polym Degrad Stab. 2016;131:132-144

24. Burg KJL, Shalaby SW. Physicochemical changes in degrading polylactide films. J Biomater Sci. 1998;9:15-29

25. Carli LN, Daitx TS, Soares GV, Crespo JS, Mauler RS. The effects of silane coupling agents on the properties of PHBV/halloysite nanocomposites. Applied Clay Science. DOI:https://doi.org/10.1016/j.clay.2013.11.032

26. Carrasco F, Gámez-Pérez J, Santana OO, Maspoch ML. Processing of poly(lactic acid)/organomontmorillonite nanocomposites: Microstructure, thermal stability and kinetics of the thermal decomposition. Chem Eng J. 2011;178:451-460

27. Carrasco F, Pagès P, Gámez-Pérez J, Santana OO, Maspoch ML. Processing of poly(lactic acid): Characterization of chemical structure, thermal stability and mechanical properties. Polym Degrad Stab. 2010;95:116-125

28. Cartier L, Okihara T, Ikada Y, Tsuji H, Puiggali J, Lotz B. Epitaxial crystallization and crystalline polymorphism of polylactides. Polymer. 2000;41:8909-8919

29. Castro-Aguirre E, Iñiguez-Franco F, Samsudin H, Fang X, Auras R. Poly(lactic acid)—Mass production, processing, industrial applications, and end of life. Adv Drug Deliv Rev. 2016;107:333-366 
30. Cele HM, Ojijo V, Chen H, Kumar S, Land K, Joubert T, de Villiers MFR, Ray SS. Effect of nanoclay on optical properties of PLA/clay composite films. Polym Test. $2014 ; 36: 24-31$

31. Celli A, Scandola M. Thermal properties and physical ageing of poly (1-lactic acid). Polymer. DOI:http://dx.doi.org/10.1016/0032-3861(92)90440-8

32. Chariyachotilert C, Joshi S, Selke SEM, Auras R. Assessment of the properties of poly(L-lactic acid) sheets produced with differing amounts of postconsumer recycled poly(L-lactic acid). J Plast Film Sheeting. 2012;28:314-335

33. Chen X, Han L, Zhang T, Zhang J. Influence of crystal polymorphism on crystallinity calculation of poly(l-lactic acid) by infrared spectroscopy. Vib Spectrosc. 2014;70:1-5

34. Choudalakis G, Gotsis AD. Permeability of polymer/clay nanocomposites: A review. Eur Polym J. 2009;45:967-984

35. Cocca M, Lorenzo MLD, Malinconico M, Frezza V. Influence of crystal polymorphism on mechanical and barrier properties of poly(l-lactic acid). Eur Polym J. 2011;47:1073-1080

36. Cohen MH, Turnbull D. Molecular Transport in Liquids and Glasses. J Chem Phys. DOI:http://dx.doi.org/10.1063/1.1730566

37. Conn RE, Kolstad JJ, Borzelleca JF, Dixler DS, Filer LJ, Ladu BN, Pariza MW. Safety assessment of polylactide (PLA) for use as a food-contact polymer. Food and Chemical Toxicology. DOI:http://dx.doi.org/10.1016/0278-6915(94)00145-E 
38. Cornell DD. Biopolymers in the Existing Postconsumer Plastics Recycling Stream. J Polym Environ. 2007;15:295-299

39. Cosate de Andrade MF, Souza PMS, Cavalett O, Morales AR. Life Cycle Assessment of Poly(Lactic Acid) (PLA): Comparison Between Chemical Recycling, Mechanical Recycling and Composting. J Polym Environ. 2016;24:372-384

40. Crank J: The Mathematics of Diffusion. Oxford England, Clarendon Press, 1975

41. Cruz Sanchez FA, Boudaoud H, Hoppe S, Camargo M. Polymer recycling in an open-source additive manufacturing context: Mechanical issues. Additive Manufacturing. DOI:https://doi.org/10.1016/j.addma.2017.05.013

42. Davis EM, Theryo G, Hillmyer MA, Cairncross RA, Elabd YA. Liquid Water Transport in Polylactide Homo and Graft Copolymers. ACS Appl Mater Interfaces. 2011;3:3997-4006

43. Deroiné M, Le Duigou A, Corre Y, Le Gac P, Davies P, César G, Bruzaud S. Accelerated ageing of polylactide in aqueous environments: Comparative study between distilled water and seawater. Polym Degrad Stab. 2014;108:319-329

44. Di Lorenzo ML. Calorimetric analysis of the multiple melting behavior of poly $(\mathrm{L}-$ lactic acid). J Appl Polym Sci. 2006;100:3145-3151

45. Dong Y, Marshall J, Haroosh HJ, Mohammadzadehmoghadam S, Liu D, Qi X, Lau K. Polylactic acid (PLA)/halloysite nanotube (HNT) composite mats: Influence of HNT content and modification. Composites: Part A. 2015;76:28-36 
46. Duan Z, Thomas NL, Huang W. Water vapour permeability of poly(lactic acid) nanocomposites. J Membr Sci. 2013;445:112-118

47. Farah S, Anderson DG, Langer R. Physical and mechanical properties of PLA, and their functions in widespread applications - A comprehensive review. Adv Drug Deliv Rev. 2016;107:367-392

48. Fukushima K, Tabuani D, Dottori M, Armentano I, Kenny JM, Camino G. Effect of temperature and nanoparticle type on hydrolytic degradation of poly(lactic acid) nanocomposites. Polym Degrad Stab. 2011;96:2120-2129

49. Gardette M, Thérias S, Gardette J, Murariu M, Dubois P. Photooxidation of polylactide/calcium sulphate composites. Polym Degrad Stab. 2011;96:616-623

50. Garlotta D. A Literature Review of Poly(Lactic Acid). J Polym Environ. 2001;9:6384

51. Gorrasi G, Pantani R, Murariu M, Dubois P. PLA/Halloysite Nanocomposite Films: Water Vapor Barrier Properties and Specific Key Characteristics. Macromolecular Materials and Engineering. 2014;299:104-115

52. Gupta KM, Pawar SJ. A nonlinear diffusion model incorporating edge and surface texture effects to predict absorption behaviour of composites. Materials Science and Engineering: A. 2005;412:78-82

53. Gupta MC, Deshmukh VG. Thermal oxidative degradation of poly-lactic acid. Colloid Polym Sci. 1982;260:514-517 
54. Hopmann C, Schippers S, Höfs C. Influence of recycling of poly(lactic acid) on packaging relevant properties. J Appl Polym Sci. 2015;132:n/a-n/a

55. International Organization for Standardization. ISO 13468-2: Plastics

- Determination of the total luminous transmittance of transparent materials -

Part 2: Double-beam instrument. 1999

56. Jaszkiewicz A, Bledzki AK, Duda A, Galeski A, Franciszczak P. Investigation of Processability of Chain-Extended Polylactides During Melt Processing - Compounding Conditions and Polymer Molecular Structure. Macromolecular Materials and Engineering. 2014;299:307-318

57. Kalish JP, Aou K, Yang X, Hsu SL. Spectroscopic and thermal analyses of $\alpha^{\prime}$ and $\alpha$ crystalline forms of poly(l-lactic acid). Polymer. 2011;52:814-821

58. Kawai T, Rahman N, Matsuba G, Nishida K, Kanaya T, Nakano M, Okamoto H, Kawada J, Usuki A, Honma N, Nakajima K, Matsuda M. Crystallization and Melting Behavior of Poly (1-lactic Acid). Macromolecules. 2007;40:9463-9469

59. Kister G, Cassanas G, Vert M. Effects of morphology, conformation and configuration on the IR and Raman spectra of various poly(lactic acid)s. Polymer. $1998 ; 39: 267-273$

60. Kopinke F-, Remmler M, Mackenzie K, Möder M, Wachsen O. Thermal decomposition of biodegradable polyesters-II. Poly(lactic acid). Polymer Degradation and Stability. DOI:https://doi.org/10.1016/0141-3910(96)00102-4 
61. Krishnaiah P, Ratnam CT, Manickam S. Development of silane grafted halloysite nanotube reinforced polylactide nanocomposites for the enhancement of mechanical, thermal and dynamic-mechanical properties. Appl Clay Sci. 2017;135:583-595

62. La Mantia FP, Botta L, Morreale M, Scaffaro R. Effect of small amounts of poly(lactic acid) on the recycling of poly(ethylene terephthalate) bottles. Polym Degrad Stab. 2012;97:21-24

63. Lai S, Wu S, Lin G, Don T. Unusual mechanical properties of melt-blended poly(lactic acid) (PLA)/clay nanocomposites. Eur Polym J. 2014;52:193-206

64. Lecouvet B, Gutierrez JG, Sclavons M, Bailly C. Structure-property relationships in polyamide 12/halloysite nanotube nanocomposites. Polym Degrad Stab. 2011;96:226235

65. Leejarkpai T, Mungcharoen T, Suwanmanee U. Comparative assessment of global warming impact and eco-efficiency of PS (polystyrene), PET (polyethylene terephthalate) and PLA (polylactic acid) boxes. J Clean Prod. 2016;125:95-107

66. Lim L-, Auras R, Rubino M. Processing technologies for poly(lactic acid). Prog Polym Sci. 2008;33:820-852

67. Ling X, Spruiell JE. Analysis of the complex thermal behavior of poly(L-lactic acid) film. I. Samples crystallized from the glassy state. J Polym Sci B Polym Phys. $2006 ; 44: 3200-3214$

68. Liu M, Jia Z, Jia D, Zhou C. Recent advance in research on halloysite nanotubespolymer nanocomposite. Progress in Polymer Science.

DOI:https://doi.org/10.1016/j.progpolymsci.2014.04.004 
69. Liu M, Zhang Y, Zhou C. Nanocomposites of halloysite and polylactide. Appl Clay Sci. 2013;75-76:52-59

70. Lorenzo V, Pereña JM. Microhardness, a non destructive test applied to polymers. Curr Trends Polym Sci. 1999;4:65-76

71. McLauchlin AR, Ghita O, Gahkani A. Quantification of PLA contamination in PET during injection moulding by in-line NIR spectroscopy. Polym Test. 2014;38:46-52

72. Meaurio E, López-Rodríguez N, Sarasua JR. Infrared Spectrum of Poly(l-lactide): Application to Crystallinity Studies. Macromolecules. 2006;39:9291-9301

73. Meaurio E, Zuza E, López-Rodríguez N, Sarasua JR. Conformational Behavior of Poly(1-lactide) Studied by Infrared Spectroscopy. J Phys Chem B. 2006;110:5790-5800

74. Meng Q, Heuzey M, Carreau PJ. Control of thermal degradation of polylactide/clay nanocomposites during melt processing by chain extension reaction. Polym Degrad Stab. 2012;97:2010-2020

75. Meng X, Shi G, Wu C, Chen W, Xin Z, Shi Y, Sheng Y. Chain extension and oxidation stabilization of Triphenyl Phosphite (TPP) in PLA. Polym Degrad Stab. $2016 ; 124: 112-118$

76. Molinaro S, Cruz Romero M, Boaro M, Sensidoni A, Lagazio C, Morris M, Kerry J. Effect of nanoclay-type and PLA optical purity on the characteristics of PLA-based nanocomposite films. J Food Eng. 2013;117:113-123

77. Mülhaupt R. Green Polymer Chemistry and Bio-based Plastics: Dreams and Reality. Macromol Chem Phys. 2013;214:159-174 
78. Murariu M, Dubois P. PLA composites: From production to properties. Adv Drug Deliv Rev. 2016;107:17-46

79. Mutsuga M, Kawamura Y, Tanamoto K. Migration of lactic acid, lactide and oligomers from polylactide food-contact materials. Food Additives \& Contaminants: Part A. 2008;25:1283-1290

80. Nascimento L, Gamez-Perez J, Santana OO, Velasco JI, Maspoch ML, FrancoUrquiza E. Effect of the Recycling and Annealing on the Mechanical and Fracture Properties of Poly(Lactic Acid). J Polym Environ. 2010;18:654-660

81. Niaounakis M: Biopolymers Reuse, Recycling, and Disposal, ed 1. Oxford, William Andrew Publishing, 2013

82. Nielsen LE. Models for the Permeability of Filled Polymer Systems. Journal of Macromolecular Science: Part A - Chemistry. 1967;1:929-942

83. Nunes RW, Martin JR, Johnson JF. Influence of molecular weight and molecular weight distribution on mechanical properties of polymers. Polymer Engineering \& Science. $1982 ; 22: 205-228$

84. Pan P, Kai W, Zhu B, Dong T, Inoue Y. Polymorphous Crystallization and Multiple Melting Behavior of Poly(l-lactide): Molecular Weight Dependence. Macromolecules. 2007;40:6898-6905

85. Pan P, Zhu B, Kai W, Dong T, Inoue Y. Polymorphic transition in disordered poly(L-lactide) crystals induced by annealing at elevated temperatures. Macromolecules. 2008;41:4296-4304 
86. Papageorgiou GZ, Achilias DS, Nanaki S, Beslikas T, Bikiaris D. PLA nanocomposites: Effect of filler type on non-isothermal crystallization. Thermochim Acta. 2010;511:129-139

87. Paul DR, Robeson LM. Polymer nanotechnology: Nanocomposites. Polymer. DOI:https://doi.org/10.1016/j.polymer.2008.04.017

88. Paul MA, Delcourt C, Alexandre M, Degée P, Monteverde F, Dubois P. Polylactide/montmorillonite nanocomposites: study of the hydrolytic degradation. Polym Degrad Stab. 2005;87:535-542

89. Paul M, Alexandre M, Degée P, Henrist C, Rulmont A, Dubois P. New nanocomposite materials based on plasticized poly(l-lactide) and organo-modified montmorillonites: thermal and morphological study. Polymer. 2003;44:443-450

90. Perego G, Cella GD, Bastioli C. Effect of molecular weight and crystallinity on poly(lactic acid) mechanical properties. J Appl Polym Sci. 1996;59:37-43

91. Piemonte V. Bioplastic Wastes: The Best Final Disposition for Energy Saving. J Polym Environ. 2011;19:988-994

92. Pillin I, Montrelay N, Bourmaud A, Grohens Y. Effect of thermo-mechanical cycles on the physico-chemical properties of poly(lactic acid). Polym Degrad Stab. 2008;93:321-328

93. PlasticsEurope. Plastics - the Facts 2016: An analysis of European plastics production, demand and waste data. 2016;2016 
94. Pluta M, Jeszka JK, Boiteux G. Polylactide/montmorillonite nanocomposites:

Structure, dielectric, viscoelastic and thermal properties. Eur Polym J. 2007;43:28192835

95. Puiggali J, Ikada Y, Tsuji H, Cartier L, Okihara T, Lotz B. The frustrated structure of poly(1-lactide). Polymer. 2000;41:8921-8930

96. Qi F, Tang M, Chen X, Chen M, Guo G, Zhang Z. Morphological structure, thermal and mechanical properties of tough poly(lactic acid) upon stereocomplexes. Eur Polym J. 2015;71:314-324

97. Raquez J, Habibi Y, Murariu M, Dubois P. Polylactide (PLA)-based nanocomposites. Prog Polym Sci. 2013;38:1504-1542

98. Rathi S, Kalish JP, Coughlin EB, Hsu SL. Utilization of Oligo(lactic acid) for Studies of Chain Conformation and Chain Packing in Poly(lactic acid). Macromolecules. 2011;44:3410-3415

99. Reddy MM, Vivekanandhan S, Misra M, Bhatia SK, Mohanty AK. Biobased plastics and bionanocomposites: Current status and future opportunities. Prog Polym Sci. 2013;38:1653-1689

100. Rhim J, Hong S, Ha C. Tensile, water vapor barrier and antimicrobial properties of PLA/nanoclay composite films. LWT - Food Science and Technology. 2009;42:612617

101. Rossi V, Cleeve-Edwards N, Lundquist L, Schenker U, Dubois C, Humbert S, Jolliet O. Life cycle assessment of end-of-life options for two biodegradable packaging 
materials: sound application of the European waste hierarchy. J Clean Prod. 2015;86:132-145

102. Rutherford SW, Do DD. Review of time lag permeation technique as a method for characterisation of porous media and membranes. Adsorption. 1997;3:283-312

103. Scaffaro R, Botta L, Passaglia E, Oberhauser W, Frediani M, Di Landro L. Comparison of different processing methods to prepare poly(lactid acid)-hydrotalcite composites. Polymer Engineering \& Science. 2014;54:1804-1810

104. Scaffaro R, Sutera F, Mistretta MC, Botta L, La Mantia FP. Structure-properties relationships in melt reprocessed PLA/hydrotalcites nanocomposites . Express Polym Lett. 2017;11:555-564

105. Sin LT, Rahmat AR, Wan Azian Wan, Abdul Rahman: Polylactic Acid: PLA Biopolymer Technology and Applications, ed 1. United Kingdom, Elsevier, 2012 106. Sinha Ray S, Bousmina M. Biodegradable polymers and their layered silicate nanocomposites: In greening the 21st century materials world. Prog Mater Sci. 2005;50:962-1079

107. Sinha Ray S, Okamoto M. Polymer/layered silicate nanocomposites: a review from preparation to processing. Progress in Polymer Science. 2003;28:1539-1641

108. Soroudi A, Jakubowicz I. Recycling of bioplastics, their blends and biocomposites: A review. Eur Polym J. 2013;49:2839-2858 
109. Stevels WM, Ankoné MJK, Dijkstra PJ, Feijen J. Kinetics and Mechanism of 1Lactide Polymerization Using Two Different Yttrium Alkoxides as Initiators. Macromolecules. 1996;29:6132-6138

110. Takamura M, Nakamura T, Takahashi T, Koyama K. Effect of type of peroxide on cross-linking of poly(1-lactide). Polym Degrad Stab. 2008;93:1909-1916

111. Tuna B, Ozkoc G. Effects of Diisocyanate and Polymeric Epoxidized Chain Extenders on the Properties of Recycled Poly(Lactic Acid). Journal of Polymers and the Environment. 2016:1-11

112. Vassiliou AA, Chrissafis K, Bikiaris DN. In situ prepared PET nanocomposites: Effect of organically modified montmorillonite and fumed silica nanoparticles on PET physical properties and thermal degradation kinetics. Thermochim Acta. 2010;500:2129

113. Yang S, Wu Z, Yang W, Yang M. Thermal and mechanical properties of chemical crosslinked polylactide (PLA). Polym Test. 2008;27:957-963

114. Yarahmadi N, Jakubowicz I, Enebro J. Polylactic acid and its blends with petroleum-based resins: Effects of reprocessing and recycling on properties. J Appl Polym Sci. 2016;133:n/a-n/a

115. Yasuniwa M, Tsubakihara S, Sugimoto Y, Nakafuku C. Thermal analysis of the double-melting behavior of poly(L-lactic acid). J Polym Sci B Polym Phys. 2004;42:2532 
116. Zaidi L, Kaci M, Bruzaud S, Bourmaud A, Grohens Y. Effect of natural weather on the structure and properties of polylactide/Cloisite 30B nanocomposites. Polym Degrad Stab. 2010;95:1751-1758

117. Żenkiewicz M, Richert J, Rytlewski P, Moraczewski K, Stepczyńska M, Karasiewicz T. Characterisation of multi-extruded poly(lactic acid). Polym Test. $2009 ; 28: 412-418$

118. Zhang J, Duan Y, Sato H, Tsuji H, Noda I, Yan S, Ozaki Y. Crystal Modifications and Thermal Behavior of Poly(l-lactic acid) Revealed by Infrared Spectroscopy. Macromolecules. 2005;38:8012-8021

119. Zhang J, Tashiro K, Tsuji H, Domb AJ. Disorder-to-Order Phase Transition and Multiple Melting Behavior of Poly(l-lactide) Investigated by Simultaneous Measurements of WAXD and DSC. Macromolecules. 2008;41:1352-1357 120. Zhang Y, Wang C, Du H, Li X, Mi D, Zhang X, Wang T, Zhang J. Promoting crystallization of polylactide by the formation of crosslinking bundles. Materials Letters. DOI:https://doi.org/10.1016/j.matlet.2013.12.005 